\title{
LONG-RANGE DISPERSAL BEHAVIOUR AND SPATIAL DISTRIBUTION MODELLING OF ADULT MOSQUITOES IN THE WINNIPEG REGION
}

By MARTINE ELySE BALCAEN

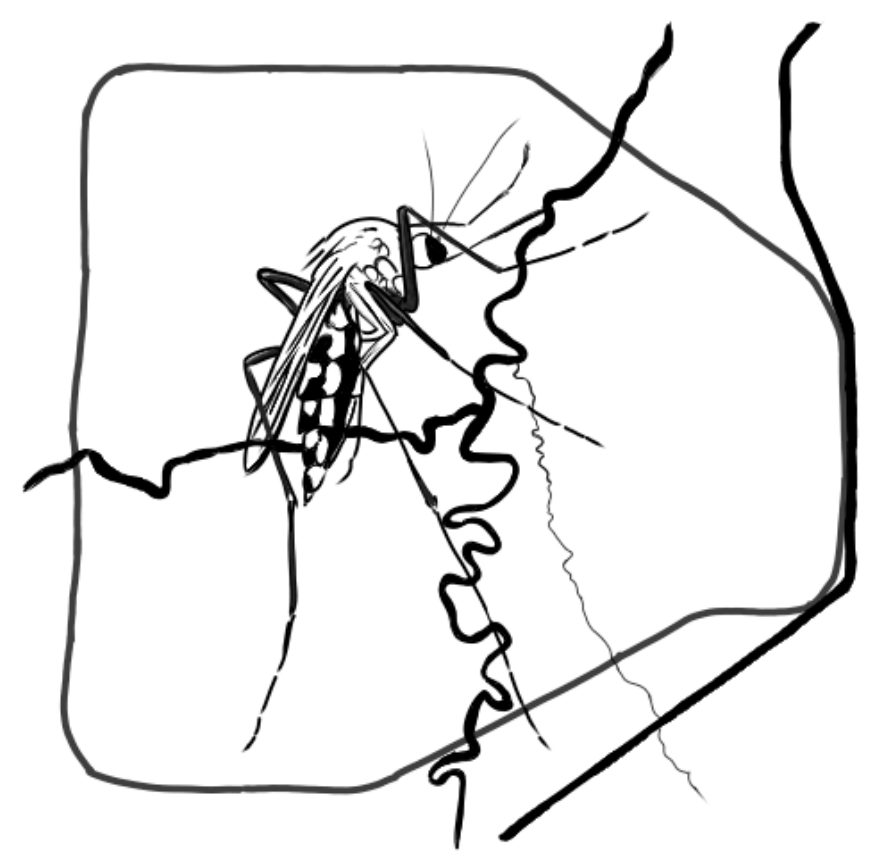

The University of Winnipeg Department of Biological Sciences

Winnipeg, Manitoba, Canada

A thesis submitted to the Faculty of Graduate Studies in partial fulfillment of the requirements for a Master of Science in Bioscience, Technology and Public Policy

December 2020 


\section{ABSTRACT}

Mosquitoes are present in virtually every nation worldwide, acting as both a vector for many serious pathogens, and as a nuisance because of their blood-feeding behaviours. As a principle of Integrated Mosquito Management (IMM), pre-emptive rather than reactive mosquito control measures are recommended and have been shown to be effective in suppressing mosquito populations. However, pre-emptive actions require insight into the spatial dynamics of mosquitoes to be effective, as mosquito dispersal behaviour is broadly influenced by local environmental conditions and species physiology.

This research project was designed to investigate the dispersal behaviour and landscape ecology of adult mosquitoes in the Winnipeg region in central Canada. In Manitoba, mosquitoes primarily present an annoyance rather than a public health risk, though a risk of exposure to several mosquito-borne diseases persists in southern Manitoba. As part of the Winnipeg's long-standing IMM program, the city maintains a mosquito control buffer zone extending approximately $10 \mathrm{~km}$ beyond the city limits. Within this zone, a surveillance program is implemented for adult and larval mosquitoes and larviciding operations occur when necessary. However, there is a lack of local evidence to justify the size of this zone, and literature addressing the establishment of effective buffer zones for mosquito control is nearly non-existent. Assessment of the potential effectiveness of a buffer zone requires information on mosquito flight ranges and dispersal behaviours, as well as knowledge of their overall distribution. This study 1) demonstrates two approaches that are commonly used to characterize mosquito behaviour for the purpose of optimizing control measures: mark-release-recapture (MRR) experiments and spatial distribution modelling, and 2) uses the results of these approaches to infer the potential effectiveness of the mosquito control buffer zone surrounding Winnipeg.

Mark-release-recapture studies have long been used to measure mosquito flight distances and to investigate the environmental drivers of dispersal movement. In this study, field-sourced larval mosquitoes were reared to adulthood and marked with fluorescent dust prior to release. I hypothesized that mosquitoes would actively disperse towards areas with higher moisture profiles, such as those with dense canopy cover or near bodies of water. Additionally, I predicted that females would orientate toward areas with high densities of 
their preferred hosts. With Winnipeg mosquito buffer in mind, these experiments were designed to a) establish the flight ranges of common mosquito species, and b) discern the influence of landscape-based variables on adult mosquitoes dispersing into the urban areas of Winnipeg from the peri-urban outskirts.

Recaptured mosquitoes included primarily Aedes vexans, Culiseta inornata, and Coquillettidia perturbans, all of which were recaptured $3 \mathrm{~km}$ or more from a single release site located on the southern edge of the City of Winnipeg within a few days of release. Female Ae. vexans were found to commonly travel more than $3 \mathrm{~km}$ following release, and male recaptures were often observed several kilometres from the release site. A few female Ae. vexans were recaptured over $15 \mathrm{~km}$ away, but the lifetime flight range for most (90\%) is estimated to be over $8 \mathrm{~km}$. Female Cs. inornata movement was in part influenced by the presence of mammalian livestock. While too few $C q$. perturbans were recaptured to estimate mean flight distance or total flight range, in two separate events, marked individuals were recaptured over $26 \mathrm{~km}$ from the release site. Generally, mosquitoes appeared to be more prevalent in areas with extensive vegetative cover, and findings suggest these areas may act as corridors that facilitate dispersal into urban areas.

Remote sensing data and spatial models created with Geographic Information Systems (GIS) platforms are increasingly being used to help clarify landscape-wide patterns of mosquito distribution. The approach presented here used nine consecutive years of trap data from Winnipeg's mosquito surveillance program to model mosquito distribution in unsampled areas. Landscape-based variables such as distance to nearest river, land cover and land use classes, as well as vegetation and wetness indices were extracted for the study area using Sentinel-2 satellite imagery at a resolution of $10 \mathrm{~m}$. Circular zonal areas ("buffer zones", though not to be confused with the mosquito control buffer zone surrounding Winnipeg) generated at varying distances around traps were used to characterize habitats in terms of these variables. These were then used as explanatory variables in random forest regressions iterated to identify key predictors of mosquito distribution. The maps produced from the final models identified "hotspots" within the Winnipeg area several common local mosquito species. I hypothesized that mosquito 
populations would be densest in areas with high moisture profiles, such as vegetated regions near rivers, as was implied by the MRR experiment outcomes.

Hotspots for Ae. vexans and Cx. restuans confirmed this hypothesis, as these were clustered within riparian areas closest to rivers. Conversely, population hotspots for $C X$. tarsalis were located near or beyond city limits. No consistent trends were identified for Ae. dorsalis. Influence of certain vegetation-based land cover classes such as cultivated land, grass and forest were more important in predicting mosquito distribution at larger scales (500 to $1000 \mathrm{~m}$ ) in comparison to land use classes such as commercial or industrial areas which influenced mosquito distributions at smaller scales (50 to $100 \mathrm{~m}$ ). These support the hypothesis that relationships between mosquitoes and their surroundings extend beyond the reach of their olfactory or visual senses and that larger scale landscape factors also influence mosquito movement to a significant extent.

Based on these findings, we can infer that many mosquito species are capable of dispersing distances that justify the extent of the current buffer zone surrounding the Winnipeg city limits. The results from the MRR experiments demonstrated that these mosquitoes could disperse several kilometres into the city from outside its limits. However, the MRR experiments were not designed to fully address the directionality of their movements, and mosquitoes may be dispersing towards rural areas as well. The results of both the spatial distribution models and the MRR experiments provided similar insights regarding habitat preferences. Most prominently, the distribution and dispersal patterns of Ae. vexans suggests that mosquitoes may be using vegetated riverbanks as corridors for dispersal. Additionally, the risk of encountering the medically important species $C x$. tarsalis increases with greater distances from the city center. Both these findings indicate that the diversity in habitat preference for Winnipeg's mosquitoes would necessitate thorough monitoring and treatment of larval habitats to prevent most mosquitoes from immigrating into the city. This may be impractical, nay impossible given the nature of the breeding habitats preferred by Ae. vexans (soils with intermittent flooding) and Cx. tarsalis (small and discrete artificial containers) and the difficulty associated with treating these. Regardless, these results illustrate an improved understanding of the landscape ecology of mosquitoes in the Winnipeg region. 


\section{ACKNOWLEDGMENTS}

I put a lot of care into writing this thesis, with the belief that it will inevitably outlast me and so would like to pass on my gratitude to the following people: Richard Westwood, who is an exceptionally great mentor; my committee members and collaborators, Joni Storie, Rob Anderson, and Ken Nowalsky; my parents, sisters, friends, and family in Calgary, who were always supportive of my decision to move to Winnipeg and study mosquitoes; Brendan Brooks; the staff at the City of Winnipeg Insect Control Branch, and tree nursery for their resources and help; my summer field assistants, Justis Henault, Stephen Kurz, Stefanie Sheard, Danéa Gauthier-Wiebe, Sarah Teillet, and Benoît Morham; all those who agreed to host a mosquito trap; my peers at the UW and at the UofM; Kateryn Rochon; Jeffrey Marcus; Alana Westwood; J. Carleton Ayer; those who lived with me throughout this process; Alexandra Elbakyan; Brenden Searing from Staten Island; the Mezzanine Crew; and all those whom I have cited, as we all stand on the shoulders of giants.

I would lastly like to thank my students, as I would not be the same without them. The work presented in this thesis began in May 2016 and took me 4.5 years to complete. I completed my field work and graduate coursework by the winter of 2018. In the fall of 2018, I taught for the first time as an adjunct professor and consumed my time with pedagogy and course preparation. While it delayed the completion of my research, it was ultimately my time teaching that made me certain that I needed to finish this work. 


\section{TABLE OF CONTENTS}

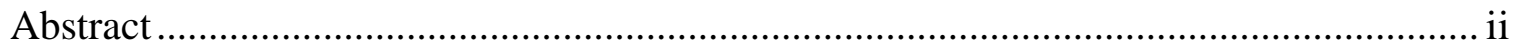

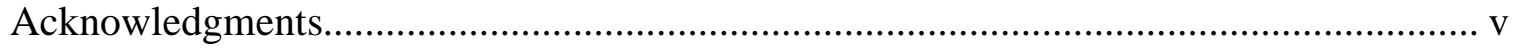

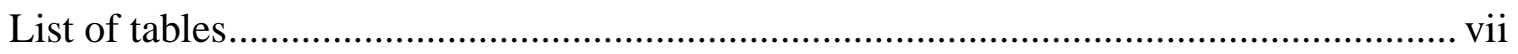

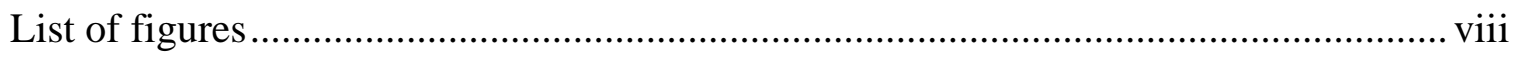

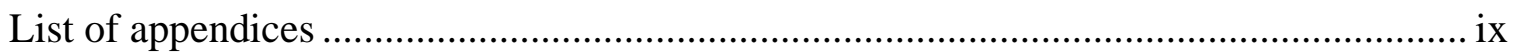

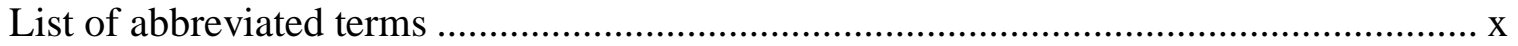

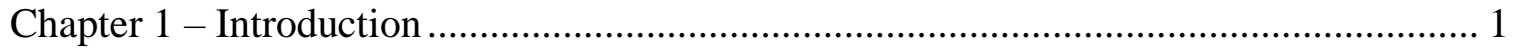

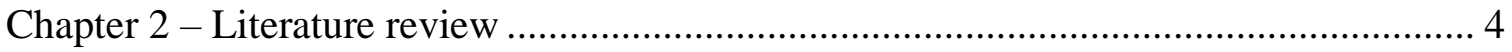

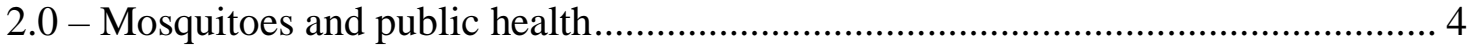

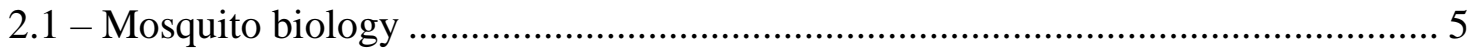

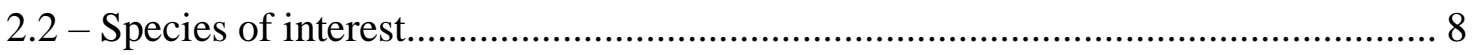

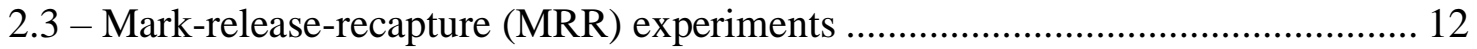

2.4 - Spatial analysis in the context of mosquito control.......................................... 25

Chapter 3 - Mark-release-recapture studies .......................................................... 42

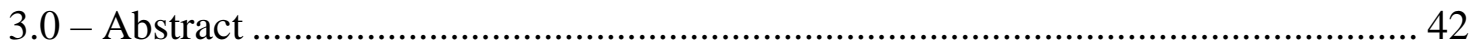

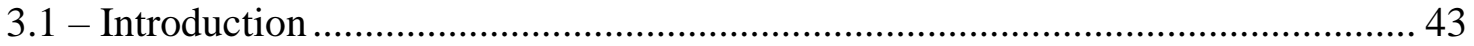

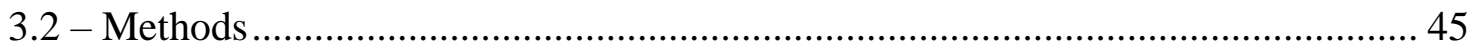

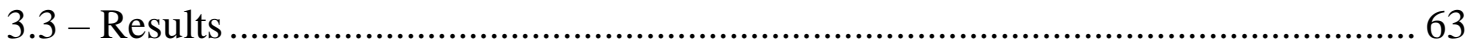

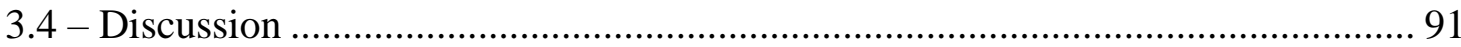

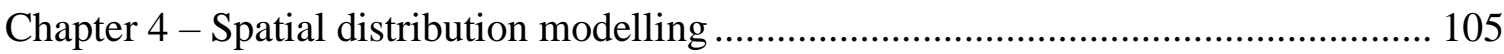

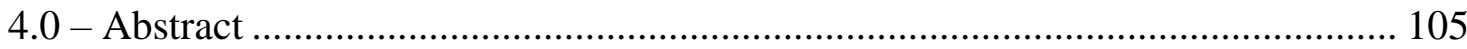

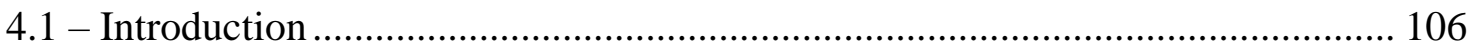

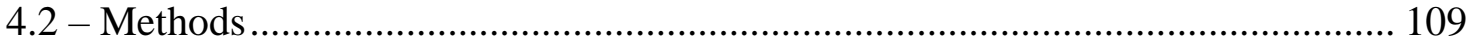

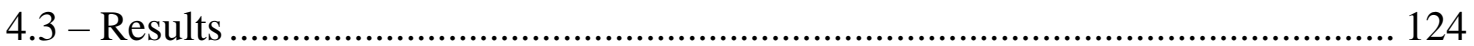

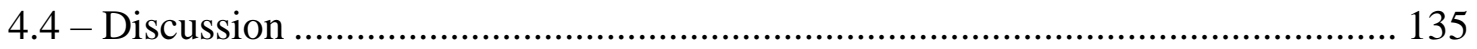

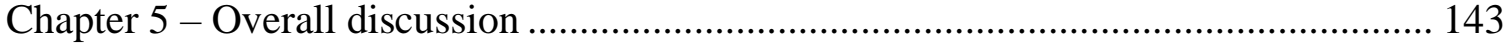

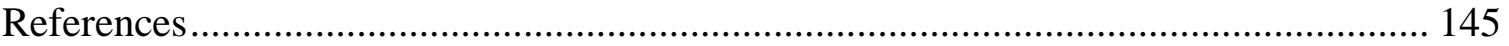

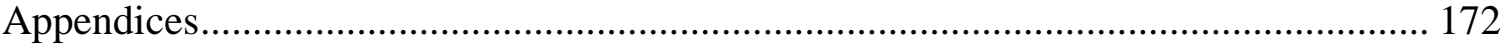




\section{LIST OF TABLES}

Table 1. Review of mark-release-recapture studies of Aedes vexans ............................ 22

Table 2. Operation timeline for traps exclusive to MRR experiment (2016) .................. 55

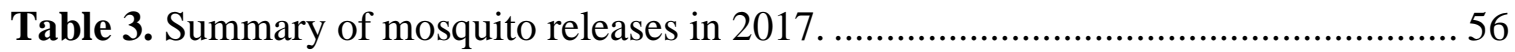

Table 4. Operation timeline for traps exclusive to MRR experiment (2017) ................. 59

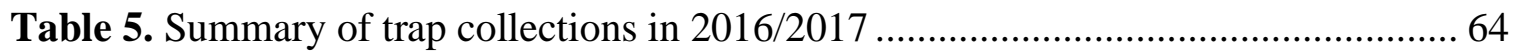

Table 6. Species and sex breakdown of recaptured mosquitoes in 2016 ........................ 65

Table 7. Example of calculating MDT with correction for unequal trap density ........... 74

Table 8. Summary of analyses to determine MDT and flight ranges ........................... 75

Table 9. Summary of mosquito releases in 2017 by colour ...................................... 76

Table 10. Species and sex breakdown of recaptured mosquitoes in 2017 ..................... 78

Table 11. Summary of analyses to determine MDT and flight ranges (full data)........... 89

Table 12. Summary of analyses to determine MDT and flight ranges $(<5 \mathrm{~km}) \ldots \ldots \ldots \ldots . . . .90$

Table 13. Summary of dust coverage on recaptured mosquitoes (2017) ....................... 97

Table 14. Description of LULC classes used in landscape classification .................... 117

Table 15. Confusion Matrix results for the LULC classification................................. 119

Table 16. Summary of best-performing Random Forest regression models by species 128 


\section{LIST OF FIGURES}

Figure 1. Region studied in MRR experiments and analysis of archival records.......... 46

Figure 2. Empty rearing pools at the release site prior to marking (2017) ..................... 48

Figure 3. Water-filled rearing pools with organic content $(2016,2017)$........................ 49

Figure 4. Photo and schematic of New Jersey light trap........................................... 50

Figure 5. Battery operated light trap hung in tree ................................................. 51

Figure 6. Location of collection site, release site and light traps (2016) ........................ 53

Figure 7. Location of collection site, release site and light traps (2017) ........................ 57

Figure 8. Example schematic of trapping annuli within study area .............................. 62

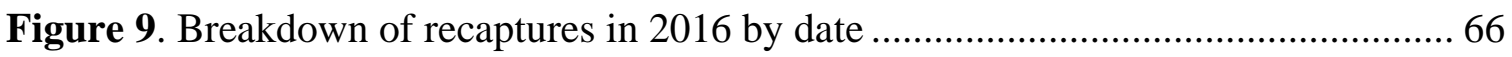

Figure 10. Recovery pattern of recaptured mosquitoes in 2016 .................................. 68

Figure 11. Female recaptures by distance and time since release (2016) ...................... 70

Figure 12. Male recaptures by distance and time since release (2016)......................... 71

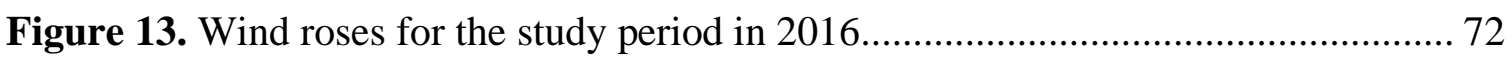

Figure 14. Boxplot comparing wind speeds for the study period in $2016 \ldots \ldots \ldots \ldots \ldots \ldots \ldots . . . . . . . . . .73$

Figure 15. Cumulative proportions of female Ae. vexans ER by recapture distance....... 77

Figure 16. Breakdown of recaptures in 2017 by date ................................................. 79

Figure 17. Breakdown of recaptures in 2017 by time elapsed since release ................. 80

Figure 18. Recovery pattern of recaptured mosquitoes in 2017 ................................ 82

Figure 19. Female recaptures by distance and time since release $(2017)$...................... 83

Figure 20. Male recaptures by distance and time since release (2017).......................... 84

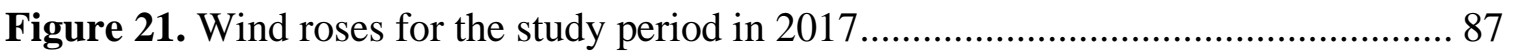

Figure 22. Boxplot comparing wind speeds for the study period in 2017 .................... 88

Figure 23. Micrographs of marked mosquitoes with extent of dust coverage ................ 95

Figure 24. Workflow summary for generating species-specific mosquito risk maps.... 110

Figure 25. Locations of NJLTs operating concurrently from 2007 to 2015 ................. 111

Figure 26. Segmented $10 \mathrm{~m}$ resolution false colour composite image of study area..... 118

Figure 27. LULC maps generated from object-based landscape classification ............. 121

Figure 28. Risk map for Aedes vexans derived from TI-based model ......................... 126

Figure 29. Risk map for Aedes vexans derived from HM-based model ....................... 127

Figure 30. Risk map for Culex tarsalis derived from TI-based model ......................... 129

Figure 31. Risk map for Culex tarsalis derived from HM-based model ...................... 130

Figure 32. Risk map for Culex restuans derived from TI-based model ...................... 131

Figure 33. Risk map for Culex restuans derived from HM-based model...................... 132

Figure 34. Risk map for Aedes dorsalis derived from TI-based model ........................ 133

Figure 35. Risk map for Aedes dorsalis derived from HM-based model ..................... 134

Figure 36. Risk maps for Ae. vexans at neighbourhood scale.................................... 142 


\section{LIST OF APPENDICES}

Appendix I. List of mosquito species collected by ICB within the Winnipeg area....... 172

Appendix II. Descriptions of all trap locations used in MRR experiments................... 173

Appendix III. Marked mosquitoes released per rearing pool (2016) ............................ 175

Appendix IV. Marked mosquitoes released per rearing pool (2017) ............................ 176

Appendix V. Precipitation, air temperature, humidity, and wind velocity (2016) ........ 177

Appendix VI. Precipitation, air temperature, humidity, and wind velocity (2017)....... 179

Appendix VII. Daily breakdown of recaptured mosquitoes (2016) ............................. 181

Appendix VIII. Number of marked and unmarked mosquitoes per trap (2016) ........... 183

Appendix IX. List of recaptured mosquitoes in 2016 ............................................... 185

Appendix X. Detailed summary of recaptured mosquitoes by colour (2017) ............... 187

Appendix XI. Chronological summary of recaptured mosquitoes (2017).................... 190

Appendix XII. Summary of recaptured mosquitoes (2017) ………………………....... 191

Appendix XIII. Mosquitoes collected per 2 km interval from release site (2017)....... 192

Appendix XIV. List of recaptured mosquitoes (2017) ................................................ 194

Appendix XV. Recaptured mosquitoes by species and colour (2017) .......................... 201

Appendix XVI. Summary of trap site environments .................................................. 203

Appendix XVII. Summary of mosquito activity by trap and species (TI and HM) ...... 204

Appendix XVIII. Yearly coefficients of variation of proportional trap totals .............. 205

Appendix XIX. Yearly coefficients of variation of the inverses of total trap counts .... 206 


\section{LIST OF ABBREVIATED TERMS}

$\begin{array}{ll}\text { CF } & \text { correction factor } \\ \text { CV } & \text { coefficient of variation } \\ \text { FR } & \text { flight range } \\ \text { GIS } & \text { geographic information system } \\ \text { GPS } & \text { global positioning system } \\ \text { HM } & \text { harmonic mean } \\ \text { LULC } & \text { land use and land cover } \\ \text { ICB } & \text { Insect Control Branch } \\ \text { IMM } & \text { Integrated Mosquito Management } \\ \text { MBD } & \text { mosquito-borne disease } \\ \text { MDT } & \text { mean distance travelled } \\ \text { MRR } & \text { mark-release-recapture } \\ \text { MSE } & \text { mean Squared Error } \\ \text { NDVI } & \text { normalized Difference Vegetation Index } \\ \text { NDVI } & \text { normalized Difference Wetness Index } \\ \text { NIR } & \text { near-Infrared } \\ \text { NJLT } & \text { New Jersey light trap } \\ \text { OOB } & \text { out of bag } \\ \text { PCA } & \text { principal component analysis } \\ \text { RF } & \text { Random Forest } \\ \text { RS } & \text { Remote sensing } \\ \text { SVM } & \text { support vector machine } \\ \text { TI } & \text { trap index } \\ \text { VIF } & \text { variance inflation factor }\end{array}$




\section{CHAPTER 1 - INTRODUCTION}

Mosquitoes are present in virtually every nation worldwide and act as both a vector for many serious pathogens, and a nuisance as the consequence of their blood-feeding behaviours. Globally, mosquito-borne illnesses are responsible for over one million human deaths annually (WHO 2019) and place a significant strain on many economies and greatly increase public health costs (Gubler 2002a; Barber et al. 2010; Utz et al. 2018). Even in regions where some mosquito species persist primarily as a nuisance to the public, such as the study area in Manitoba (Canada), transmission risk of West Nile virus, Western Equine Encephalitis, and other illnesses is still present. Additionally, nuisance and exposure risk to life-threatening mosquito-borne illnesses may negatively affect property value, tourism, and economic development (AMCA 2017).

Many organizations with responsibilities for mosquito control approach this challenge using integrated mosquito management (IMM), which employs of a broad range of practices to control mosquito populations. Within an IMM framework, mosquito control makes use of conventional insecticide usage (adulticide) alongside surveillance, mapping, establishment of action thresholds for control decisions, reduction of immature mosquitoes and their source habitat, resistance monitoring, public outreach, and biological or genetic control agents. The goal of this strategy focuses on long-term suppression of mosquito populations in a manner which is environmentally sustainable and results in the least economic injury (AMCA 2017).

Increasingly, mapping and analysis of spatial data using Geographic Information Systems (GIS) platforms is being recognized as a powerful tool in IMM to enhance surveillance and control operations. A recent global emergence and resurgence of mosquito-borne diseases, such as dengue, zika and West Nile virus (Gubler 2002b; Vega Rúa and Okech 2019), has emphasized the importance of developing spatial models to predict mosquito activity and disease risk (Ostfeld et al. 2005; Winters et al. 2008; Palaniyandi 2012; Clements et al. 2013; Fouet and Kamdem 2018; Tjaden et al. 2018).

When making control decisions, spatial models should be created and tailored to an area's unique geography and consider biologically relevant information about the target 
species. As mosquitoes exhibit species-specific habitat preferences, spatial IMM models have been designed to capture their distributions with respect to climatic and landscapebased variables (Diuk-Wasser et al. 2006; Reiter and LaPointe 2007; Chuang et al. 2011; Landau and van Leeuwen 2012; Ibañez-Justicia and Cianci 2015; Zittra et al. 2017). Integration of these variables in models is essential when facing global climate change and changes in patterns of human land use.

Since the late 1970s, the City of Winnipeg's Insect Control Branch (ICB) has adopted the principles of IMM to monitor local mosquito populations and undertake mosquito control measures based on surveillance data (City of Winnipeg 2015). While advocating for public education and community outreach to aid mosquito control, the ICB's mosquito control program utilizes both larval and adult mosquito surveillance to support decisions for the application of control agents as needed. Larval and adult mosquito stages are regularly sampled and examined to monitor the presence of significant disease vectors and their pathogens.

The adult mosquito population in Winnipeg and its surrounding area is sampled using an assemblage of standard New Jersey Light Traps (NJLTs). These are used to determine the effectiveness of larvicide activities and to monitor population numbers of either nuisance or vector mosquitoes in advance of adulticide actions. Adult trap surveillance also has a secondary purpose in monitoring the species composition of local population, as well as their habitat preferences (City of Winnipeg 2015).

For surveillance of larval mosquitoes, the Winnipeg ICB currently uses a GIS (Geographic Information System) to inventory all known larval mosquito development sites within the city as well as within a "buffer" zone beyond the city's boundaries. As per the City of Winnipeg Charter Act section 134(2) (d), the city has authority to larvicide mosquito larval development sites extending up to $24 \mathrm{~km}$ past its limits (Government of Manitoba 2002). However, buffer zone operations typically extend only 8 to $12 \mathrm{~km}$ (usually $<10 \mathrm{~km}$ ) beyond city boundaries, as resources and time do not normally allow for the maximum buffer size to be maintained (City of Winnipeg 2019). Regardless, there is little local evidence to support the effectiveness of this buffer area at any size, and literature on the topic is limited. 
When coupled with the use of a GIS platform to derive maps, insights from analyses of archival mosquito surveillance data can help to clarify spatial patterns of adult mosquito distribution. Geographic visualization of analysis results may allow for better prioritization of mosquito control measures to areas where nuisance and risk for pathogen transmission are likely to be highest. Additionally, as mosquito movement results in their distribution across an urban environment, these models can be supported by insights from MRR experiments. An improved understanding of the movement and landscape ecology of mosquitoes will allow for more effective mosquito management operations. Additionally, it may provide additional information to the public, government and other stakeholders concerning optimal approaches to mosquito control.

Within this thesis, Chapter 2 will serve as an informational primer to the experiments and analyses discussed in Chapters 3 and 4, which showcase the process and outcomes of the MRR experiments and the GIS analyses, respectively. The objectives for Chapter 3 are as follows: a) To perform mark-release-recapture (MRR) experiments and discern the general influence of landscape-based (i.e., land cover and use) variables on adult mosquitoes dispersing into the Winnipeg urban area from the periphery of the city; and b) to assess whether the current buffer zone, in which ICB staff monitor and control larval populations around the Winnipeg urban area, is sufficient with respect to the dispersal capabilities of common mosquitoes in the region. For Chapter 4, objectives were to: a) determine the key predictors of adult mosquito distribution in relation to landscape characteristics such as tree cover, proximity to rivers and built areas; and to: b) derive maps from these predictors that identify the locations that are likeliest to harbour the most abundant mosquito populations.

Studies from both chapters converge on a single hypothesis: adult mosquitoes will exhibit dispersal patterns driven by landscape-based factors. More specifically, I predict that mosquitoes will show preference in their dispersal and distribution to areas with higher moisture profiles, such as those with high vegetation cover, or near rivers, with females showing affinity towards areas with high densities of their preferred hosts. 


\section{ChAPTER 2 - LiterATURE REVIEW}

\section{0 - Mosquitoes and public health}

Mosquitoes are small, inconspicuous flies (Order: Diptera) contained in the family Culicidae within the suborder Nematocera ("thread-horned flies"). At the time of this writing, a total of 3,565 species have been described (Harbach 2013), and over a hundred, mainly of the genera Aedes Meigen, 1818, Culex Linnaeus, 1758, and Anopheles Meigen, 1818 are competent vectors of various pathogens affecting humans and other animals (Rueda 2008). Mosquito-borne diseases (MBD) have killed more people than all the wars in human history, and continue to infect and debilitate an estimated 700 million each year globally (Caraballo and King 2014). These include parasitic infections, such as malaria and filariases (e.g., dog heartworm), as well as viral diseases, such as dengue, yellow fever, chikungunya, and many viral encephalitides (Service, 2008).

While risk of mosquito-borne pathogen transmission is highest in tropical and subtropical climates, this danger is not absent from temperate regions. In the United States, significant annual economic losses in the millions of dollars are derived from the morbidity and mortality caused by West Nile virus, Eastern Equine Encephalitis virus, and LaCrosse virus, among others (Villari et al. 1995; Barrett 2014; Staples et al. 2014; Utz et al. 2018). Others have found that even in the relative absence of a significant threat to public health, nuisance mosquitoes can also affect the mental health and quality of life of the public (Worobey et al. 2013; Halasa et al. 2014).

In Manitoba, mosquitoes primarily present an annoyance rather than a public health risk, though a risk of exposure to several MBDs persists in southern Manitoba. Throughout the mid-1970s and early 1980s, outbreaks of Western Equine Encephalitis virus persisted across the prairie provinces of Canada (Alberta, Saskatchewan and Manitoba), harming both humans and horses (Sellers and Maarouf 1988). Additionally, fewer than 10 human West Nile virus cases per year on average have been reported in the last decade, though epidemic years with up to 585 recorded cases have been observed (Government of Manitoba 2018). 


\section{1 - Mosquito biology}

Unless otherwise cited, the following description of mosquito biology has been taken from comprehensive published resources by Rueda (2008), Service (2008) and Becker (2010).

Mosquitoes are insects which undergo complete metamorphosis (holometaboly) as they pass through three distinct immature aquatic stages; the egg, larva, and pupa, before their emergence as free-flying adults. Food availability and weather conditions both contribute to variable life cycle lengths within populations of the same species and between different species (Bowles and Swaby 2006).

The mechanics of oviposition (egg-laying) varies across genera, though the female adult generally lays several hundred eggs singly (as in genera Aedes and Anopheles) or in clusters known as "rafts" (as in genera in Culex and Culiseta Felt, 1904). Eggs are typically deposited on the surface of still waters, though some genera specifically prefer to oviposit on aquatic vegetation, in moist soils or in artificial containers which flood or collect water. Mosquitoes are prolific in their colonization of aquatic habitats, occupying those that are temporary or permanent, clean, or polluted, and natural or artificial, including large floodplains to small containers (e.g., tires and flower vases). In many species, eggs enter a period of suspended development (diapause) in the event of adverse environmental conditions such as extreme cold or drought. Eggs often require a specific environmental stimulus to hatch, such as a flooded environment, an increase in the day length or temperature of the air above, or a decrease in dissolved oxygen in the water below. These specific stimuli may be subject to differences between species, as well as differences between populations, which may be adapted to the hydrological characteristics of their specific environments.

Once hatched, larvae progress through four successive molting (instar) stages before developing into pupae. Larvae cannot breathe underwater and must take in air from the water's surface or from aquatic plants (e.g., in Coquillettidia Dyar, 1904 spp.). Most mosquito species filter-feed on aquatic microorganisms and other organic matter such as detritus for the duration of their larval stage (5 to 14 days depending on temperature). The pupal stage is short-lived ( 2 to 3 days, up to 12 days in cooler climates) and does not feed. 
Once development is complete, the cephalothorax splits and the adult mosquito emerges at the surface of the water.

Shortly after emergence from the pupa, mosquitoes mate in swarms where copulation takes place during flight. In this sense "site of emergence", "breeding site" and "oviposition site" are synonymous terms. Most mosquitoes only mate once, as sperm obtained by a female serves to fertilize all eggs throughout her lifetime. In most species, female mosquitoes are nonautogenous, meaning they must obtain a blood meal to complete the development of eggs prior to oviposition. Some species are truly host-specific, and are adapted only to feed on humans or other types of animals, such as mammalian or avian livestock, wildlife, or pets (Takken and Verhulst 2013). Other species are opportunistic in their host choice and may feed on a variety of hosts. Once host blood has been digested and the eggs are fully developed, the female begins to search for habitats suitable for larval development.

The average maximum flight distance of adult mosquitoes is highly variable, ranging from $50 \mathrm{~m}$ to $50 \mathrm{~km}$, depending on the species, with variation between populations within species (Verdonschot and Besse-Lototskaya 2014). While there are few exceptions, mosquito flight behaviour is termed "dispersive" and not "migratory". As described by Service (1997), true migratory behaviour in insects is 1) associated with purposeful colonization or return to a specific region and 2) mostly observed in pre-reproductive females. Nevertheless, observed "migrations" in mosquitoes are often synonymous with passive (or non-appetitive) flight behaviour, which serves no physiological need. This passive flight behaviour is frequently unidirectional and influenced primarily by wind velocity (to the degree that upwind dispersal is not possible) (Garrett-Jones 1950; Bidlingmayer 1964; Schäfer et al. 1997). Certain species are adapted to disperse during strong wind events (Service 1980), but in general the survival rate during wind-assisted dispersal is likely lower due to harsher conditions (Bell et al. 2005). Temperature, humidity and illumination levels are also influential factors in passive flight behaviour (Platt et al. 1957; Bidlingmayer 1964; Lewis and Taylor 1967).

Active dispersal, also referred to as "appetitive" or "oriented" flight behaviour, stands in contrast to passive dispersal ("non-appetitive" or "non-oriented") and comprises 
shorter daily flights to search for hosts, nectar, mates, oviposition sites, and resting areas (Verdonschot and Besse-Lototskaya 2014). This type of dispersal is sensitive to microclimate, and is thus influenced by local topography, vegetation structure and host presence or density (Verdonschot and Besse-Lototskaya 2014), though said to be relatively independent of wind direction (Bailey et al. 1965; Dow et al. 1965; Reisen and Lothrop 1995). While active dispersal is essential in understanding ecological interactions involving mosquitoes, passive flight is significant in that it may enable mosquitoes to travel further than the few kilometers generally observed by their active dispersal.

Female mosquitoes actively locate hosts, oviposition and sheltering sites primarily using airborne olfactory cues leading to chemotaxis (movement toward a potential source) (Takken and Knols 1999). While research concerning their flight orientation with respect to olfactory cues is limited, female mosquitoes have been observed to fly upwind toward the source of host odours, with this behaviour amplified with increasing concentrations of the host odorant. Geier et al. (1999) demonstrated that different host odour cues could elicit upwind flight dependent on cue-specific plume structure, consistent with the expected pattern of emission for different cues (i.e., continuous large, undisrupted emissions from the skin compared to periodic breath exhalations). These chemical cues include various odorous organic chemicals given off by host skin emanations (e.g., sweat) or products of respiration, such as carbon dioxide, lactic acid or octenol (Bowen 1991; Zwiebel and Takken 2004). Other olfactory stimuli include acetone, butanone and other phenolic or carboxylic acids (Takken 1991). Visual colour cues have also been reported to affect the close-range oviposition response of several species (McCrae 1984; Li et al. 2010), whereas warmth (Peterson and Brown 1951; Khan et al. 1968; Eiras and Jepson 1994), humidity (Olanga et al. 2010) and body mass (Smith 1956; Carnevale et al. 1978) may modify the dispersal and effect of volatile odorant cues (Takken and Verhulst 2013). Research pertaining to host-seeking behaviour with respect to specific odorant compounds has generally been restricted to species with significant medical importance, such as Aedes aegypti (Linnaeus, 1762) and Anopheles gambiae Giles, 1902. Specific host preferences will affect the distance and direction of active dispersal, as more ornithophilic species will disperse short distances vertically towards tall trees, whereas mammal-feeding species will expend more energy on horizontal dispersal (Verdonschot and Besse-Lototskaya 2014). 
However, the olfactory sensilla on the antennae which house the sensory structures responsible for detection of these cues are similar across medically important mosquito genera (Bowen 1991).

Service (1997) added that it may be most helpful to regard all flight behaviour on a continuum between appetitive and non-appetitive, given the number of variables involved. As such, the research presented in Chapters 3 and 4 considers that the observed spatial distribution of mosquitoes may result from factors pertaining to both appetitive and nonappetitive flight behaviour simultaneously. Understandings of both long-range, passive dispersal, as well as short-range, landscape-dependent active dispersal can be equally important in establishing a control strategy which is appropriate to its locality.

\section{2-Species of interest}

The mosquito nomenclature adopted in this thesis reflects a simplified taxonomic classification system reinstated by Wilkerson et al. (2015), wherein Ochlerotatus Reinert, 2000 is retained as a subgenus within the genus Aedes, instead of representing its own genus. Changes in the taxonomic rankings of the tribe Aedini Neveu-Lemaire, 1902 first proposed by Reinert (2000), and later subject to extensive morphology-based phylogenetic studies by Reinert, Harbach and Kitching (2004; 2006; 2008; 2009) have been inconsistently applied across the literature, and explicitly resisted by major journals (Weaver 2005; Reisen 2016), including journals to which the above authors have since contributed (Harbach et al. 2017).

While recognizing that progress should be made toward establishing monophyletic groups of species, the nomenclature used here aligns with the recommendations that mosquitoes should be identifiable to genus without necessitating the dissection of genitalia as is required by the new Aedini classification scheme (Savage and Strickman 2004). Confusion among the operational mosquito control community should be avoided until these taxonomic rankings are stabilized (Wilkerson et al. 2015). Where applicable, species which have been placed in the genus Ochlerotatus proposed by Reinert (2000), will be initially indicated in parentheses following the original generic designation of "Aedes". 
As of 2015, City of Winnipeg ICB staff have identified 40 mosquito species within the City of Winnipeg control zone (City of Winnipeg 2015). A full list of these species appears in Appendix I. For concision, only those which are observed in appreciable numbers in ICB trap collections (from 2007 to 2015) or are significant as disease vectors for human and livestock will be discussed. In order of relative importance, these are Aedes vexans (Meigen, 1830), Culex tarsalis Coquillett, 1896, Culex restuans Theobald, 1901, Aedes (Ochlerotatus) dorsalis (Meigen, 1830), Culiseta inornata (Williston, 1893), and Coquillettidia perturbans (Walker, 1856). Physical descriptions of these species are omitted here for brevity, but can be found in a number of keys (Carpenter and LaCasse 1955; Wood et al. 1979; Darsie and Ward 2005; Becker 2010).

\section{Aedes vexans}

Aedes vexans is often referred to as the "inland floodwater mosquito" and is a cosmopolitan species which has been collected on every continent except South America and Antarctica (Weissman 2016). It the most abundant floodwater mosquito in the Northern Hemisphere (O'Malley 1990) and often considered the most significant pest of humans and livestock in Canada (Russo 1977; Wood et al. 1979). They dramatically outnumber all other mosquito species in the Winnipeg area during the summer, with trap collections often consisting of over 90\% adult Ae. vexans (City of Winnipeg 2015, unpublished data 2007-2015).

Aedes vexans are long-lived compared to other species, with an average lifespan of three to six weeks (Horsfall et al. 1973), though some longevity studies have recaptured marked individuals up to 16 weeks after release (James and Harwood 1965). They lay their eggs in moist soils which are prone to inundation, and overwinter in the egg stage (O'Malley 1990), though there is inconclusive evidence that they may lay eggs directly on water, perhaps when suitable sites are unavailable (Headlee 1945). The mechanisms which allow female Aedes mosquitoes to locate appropriate oviposition sites are not fully understood, though it has been suggested that chemical attractants may be involved. Becker (2010) speculated that females may be driven to suitable areas by pheromone-like odours given off by either eggs already laid in the soil, or by plants associated with a specific level of moisture in the soil. 
Female Ae. vexans feed primarily on mammals but will also incidentally feed on birds (Shemanchuk 1969; Horsfall et al. 1973). Having notable long-range flight capabilities, Ae. vexans have been observed to disperse more than $8 \mathrm{~km}$ from their site of emergence (Rees 1943; Headlee 1945; Carpenter and LaCasse 1955; Brust 1980). Flight mill studies, confirmed by field data, have shown their dispersal capabilities maximized at $10 \mathrm{~km}$ to $17 \mathrm{~km}$ per night (Briegel et al. 2001), with Horsfall (1954) speculating that windassisted flight may allow them to travel several hundred kilometers over only a few days. A metadata analysis of flight capacity across multiple species ranked Ae. vexans as a strong flyer, with an average maximum flight distance of over $5 \mathrm{~km}$, independent of landscape type (Verdonschot and Besse-Lototskaya 2014).

\section{Culex tarsalis}

Culex tarsalis is commonly named the "Western Encephalitis mosquito" and can comprise $1.5 \%$ to $3 \%$ of mid-summer trap collections at their peak in Winnipeg (City of Winnipeg 2006; City of Winnipeg 2015). Though resting places vary between regions, adults overwinter in moist, protected places such as caves, storm drains, burrows and piles of organic debris or rock (Price et al. 1960; Shemanchuk 1965; Su et al. 2003). Their larvae occupy a variety of natural habitats as well as artificial water-filled containers (Wood et al. 1979), and are associated with irrigated farm and ranch lands (Moore et al. 1993).

Culex tarsalis show host preference to birds, but may bite humans and other mammals incidentally (Carpenter and LaCasse 1955), though they are rarely abundant in residential areas as observed by studies in California (Reisen, Meyer, et al. 1990; Reisen, Pfuntner, et al. 1990). Their flight range has been demonstrated to be between 0.8 to $4 \mathrm{~km}$ from their breeding site (Reeves et al. 1948), though they have been found to travel more than $25 \mathrm{~km}$ with the prevailing winds (Bailey et al. 1965; Rowley 2015). They are designated as strong fliers, with average maximum dispersal distance exceeding $13 \mathrm{~km}$, and an average flight distance of $545 \mathrm{~m}$ (Verdonschot and Besse-Lototskaya 2014).

\section{Culex restuans}

Culex restuans is sometimes known as the "white-dotted mosquito" and constitutes an average of $1.5 \%$ of adults in ICB trap collections (unpublished data, 2007-2015), with 
the highest counts (up to 10\%) appearing in late spring. As larvae, they occupy various habitats, including containers, ditches, and stream pools, and are known to prefer cooler, more shaded habitats compared to $C x$. tarsalis. For oviposition, nutrient-rich water is preferred over clean water or water already hosting larvae of other mosquito species (Walter Reed Biosystematics Unit 2020).

Culex restuans prefer avian hosts and are not considered pests of humans though they may incidentally feed on mammals (Moore et al. 1993). Flight range for this species has not been well-documented but has been reported to be approximately $2 \mathrm{~km}$ from their breeding site (Turell et al. 2005), with one study reporting flights of over $5 \mathrm{~km}$ over open water (Horsfall 1955).

\section{Culiseta inornata}

Culiseta inornata is commonly referred to as the "winter marsh mosquito", and is the dominant mosquito species in the late spring in Winnipeg, representing 60 to $80 \%$ of the trapped population in May, but are outnumbered by other species later in the summer (City of Winnipeg 2006; City of Winnipeg 2015). Various natural habitats support their larvae, including artificial containers such as used tires (Dyar 1922; McMahon et al. 2008).

Females of Cs. inornata prefer specific mammalian livestock hosts, such as horses and cows (Carpenter and LaCasse 1955), and seldom feed on human, swine or avian hosts (Anderson and Gallaway 1987). Their flight range is not well-studied, but they have been observed to travel in excess of $2 \mathrm{~km}$ from their breeding site (Hudson and Edman 1978). Other observations vary; some have claimed that their flight range is "less than 5 miles ( 8 km)" (Napa County Mosquito Abatement District, n.d.), whereas Clarke (1943) recovered an individual over $22 \mathrm{~km}$ away.

\section{Coquillettidia perturbans}

Coquillettidia perturbans, often called the "cattail mosquito" or "irritating mosquito", appear in Winnipeg from late June to late July in modest numbers, comprising approximately $1 \%$ of all trap collections (City of Winnipeg 2006; City of Winnipeg 2015). Their larvae require habitats with abundant emergent vegetation, most commonly cattails 
(Typha L. spp.) and sedges (Carex L. spp.), from which they draw oxygen with their hooklike siphons (Carpenter and LaCasse 1955; Moore et al. 1993).

Females of $C q$. perturbans primarily feed on mammals, but may opportunistically feed on bird species (Tempelis et al. 1967; Edman 1971; Magnarelli 1977; Molaei et al. 2008). Their dispersal capabilities are not well-studied, but they have been observed to be strong fliers, capable of flying many kilometres from their breeding site (Horsfall 1955), and are known to be phototropic (Morris et al. 1991). One observation cited Cq. perturbans demonstrating an average maximum distance of $3.4 \mathrm{~km}$, with an average flight distance of $1.7 \mathrm{~km}$ (Verdonschot and Besse-Lototskaya 2014).

\section{Aedes dorsalis}

Aedes dorsalis is a floodwater mosquito species and comprises 1-2\% of trap collections on average in Winnipeg (unpublished data, 2007-2015). They require flooding to hatch, similar to Ae. vexans (Carpenter and LaCasse 1955; Moore et al. 1993). In Manitoba, their larvae are most frequently found in temporary pools near areas with large mammals, their preferred hosts (Dixon and Brust 1972; Loftin et al. 1997). Roadside ditches and freshwater marshes are also common larval habitats, particularly grassy, sunlit areas (Carpenter and LaCasse 1955).

They are known to be strong fliers, and have been found several dozen kilometers from their breeding sites (Rees and Nielsen 1947). Verdonschot and Besse-Lototskaya (2014) cited several observations where the average maximum flight distance of females was $6.9 \mathrm{~km}$.

\section{3 - Mark-release-recapture (MRR) experiments}

The development of a knowledge base from which to understand the dispersal capabilities and other bionomic characteristics of pertinent mosquito species is valuable for the optimization of abatement strategies. Mark-release-recapture (MRR) studies are

commonly designed to estimate certain parameters necessary for addressing pathogen transmission risk within a given locality, such as dispersal range, population size, survival rates, blood feeding frequency and host preferences (Guerra et al. 2014a). However, they may also be used to study gonotrophic cycle duration, as well as feeding, mating or flight 
behaviours (Service 1993). Hundreds of MRR experiments have been performed since the 1950 s, contributing data on these bionomic parameters for various vector and pest species across a range of ecological settings (Guerra et al. 2014a). Exact procedures vary widely, but MRR experiments involve marking all individuals of a test population, releasing them unharmed back into their environment, and later recapturing them in traps surrounding their release site(s). The recaptured individuals are then inspected for the presence of the marking agent to differentiate them from the unmarked captures (Morris et al. 1991).

Strategies for mosquito control programs can benefit from MRR studies, as they can infer the patterns of movement for specific species and inform mosquito control programs as to where future intervention efforts would be most efficient and cost-effective (Morris et al. 1991; Russell et al. 2005). Development of these strategies frequently includes discussions regarding the size and treatment of buffer zones surrounding urban management areas (Verdonschot and Besse-Lototskaya 2014; Webb and Russell 2019). However, it is difficult to obtain evidence that mosquitoes produced outside of urban areas (ideally included within buffer zones) can present an exposure risk to those living within urban areas (Morris et al. 1991). This is especially true with mosquitoes that exhibit longrange dispersal capabilities, such as Ae. vexans (Horsfall 1954; Brust 1980).

The best ethical and operational practices (given research goals) must be considered when devising methods for sourcing, marking and recapturing mosquitoes from test populations for MRR experiments. Logistical challenges arise when studying the movement patterns of a small insect whose presence is spatially and temporally variable over a relatively large area. However, there are also two distinct ethical hazards associated with experimental releases of mosquitoes in or near urban environments. First, the possibility of an increase in the mosquito population which increases risk of exposure to biting mosquitoes in the local population and livestock; and second, the possibility that the experimental population will reproduce with the local wild population, which may affect its behaviour or resistance to control interventions (Benedict et al. 2018). Fears over an increased risk of exposure to biting mosquitoes following an experimental release can be alleviated by the intensive effort to capture adults following release. As demonstrated by Benedict et al. (2018), in over two-thirds of MRR experiments reviewed, the volume of 
unmarked mosquitoes captured was greater than the number released, resulting in a net population and risk reduction. Ecological concerns regarding the source of mosquitoes, as well as considerations related to the other components of MRR procedures will be discussed in the following sections.

\section{Mosquito sourcing}

The outcome of an MRR experiment may be influenced by the source and condition of released marked mosquitoes. Experimental mosquito releases may use field-collected individuals (either adults or larvae) or a laboratory-sourced cohort. Field-collected individuals were used in most (76\%) of the 774 experiments published between 1913 and 2010 that were reported in the mosquito MRR database compiled by Guerra et al. (2014). Using lab-sourced mosquitoes may be convenient when a release requires many same-aged individuals. However, researchers assume that by using local field-collected individuals, they minimize concerns surrounding the genetic differences in lab-sourced individuals which can affect their behaviour (Reisen et al. 1985). Additionally, lab-sourced mosquitoes which are genetically distinct from the wild population carry a risk of modifying the genetic makeup of the local population if they should produce hybrid offspring with them.

Many non-genetic characteristics of field-collected individuals also carry the risk of affecting research outcomes, including age, sex ratio, feeding status, collection location, and larval environment. The age of field-collected individuals, especially of adults, may be unknown, which can affect recapture rates and dispersal behaviour. Field-collected adults may also consist of more females due to their longevity over males, and due to biased sex ratios generally seen in trap collections (Benedict et al. 2018), though this is sometimes desirable depending on the research question(s). The feeding status of field-caught females is also of concern, as blood-fed females seeking to oviposit may disperse differently than host-seeking females. Similarly, the stage of field-caught individuals can also affect research outcomes; in comparison to individuals which had been reared from fieldcollected larvae, adult females of $C x$. tarsalis that were collected by $\mathrm{CO}_{2}$-baited traps were more easily recaptured by $\mathrm{CO}_{2}$-baited traps (Reisen and Lothrop 1995; Reisen et al. 2003). However, these findings contrast with earlier studies, in which comparatively lower 
recapture rates were not observed for mosquitoes reared from field-collected larvae (Nelson et al. 1978; Nelson and Milby 1980; Reisen et al. 1981; Reisen et al. 1992).

To avoid confounding factors associated with using field-collected adults for MRR experiments, it is preferable to collect local larval populations and rear these in the laboratory or field. Even so, it has been suggested there may be effects on survival and dispersal behaviour brought on by different rearing environments (Benedict et al. 2018). Furthermore, field-collected larvae may represent a range of instars, which may emerge at different times and require several marking and release events. Decisions made with respect to the source of mosquitoes used in MRR experiments should be aligned with the research question of the investigation.

\section{Marking procedure}

Marking of adult mosquitoes is commonly carried out with fluorescent pigments, typically powders (Brust 1980; Costantini et al. 1996; Bogojević et al. 2011; Ageep et al. 2014; Webb and Russell 2019) or dyes (Wada et al. 1969; LaBrecque et al. 1975), but is also accomplished with more resource and labour-intensive means, such as isotope labeling (Jenkins and Hassett 1951; Hamer et al. 2014; Faiman et al. 2019) and individual handmarking with paints (Macdonald et al. 1968; Conway et al. 1974; Harrington et al. 2005; Tsuda and Kamezaki 2014). The latter two methods are most often used when adults cannot be marked (when marking larvae) or if multiple distinct markings are needed, respectively. Marking with powdered fluorescent pigments is the most common method for MRR studies (Guerra et al. 2014a), preferred for its relative environmental safety, costeffectiveness, easy application (Hagler and Jackson 2001) and detection under ultraviolet (UV) light, visible for at least 30 days following release (Brust 1980; Rojas-Araya et al. 2019). It is usually applied to large numbers of adults in a confined space where the powder is scattered into the air, where adults can fly through it to be marked.

Uncertainties about the use of fluorescent powder or "dust" in insect MRR studies

primarily rest on the possibility of a) altering the behaviour or survivorship of marked individuals, and b) transfers from marked to unmarked individuals during mating or other contact following release. Many studies investigate the effects of marking insects with the use of dusts, but primarily measure the survivorship of marked specimens compared with 
those unmarked (Naranjo 1990; Corbett and Rosenheim 1996; Rhodes et al. 1998; Coviella et al. 2006; Clymans et al. 2020). Many of these studies report no adverse effects on the survival of marked insects. While some adverse effects on long-term survivorship may be found, these are likely not significant on the scale of MRR studies assessing dispersal, as most marked individuals are recaptured before their natural death (Poland et al. 2000). However, survivorship is only one of many factors that may affect dispersal behaviour. Concerns have also been raised about the desiccating effect or other unknown stressors associated with marking insects with fluorescent dusts, which could alter their dispersal behaviour (Reid and Reid 2008).

For example, over-application has been found to cause high mortality, decreased mobility and sensory organ interference in flies and beetles (Crumpacker 1974; Cook and Hain 1992). While some insects have been reported to experience adverse effects to their survival or behaviour as a result of marking with fluorescent dusts (Moffitt and Albano 1972; Dye et al. 1991; de Guzman et al. 2012), this has seldom been observed in mosquitoes. Many field studies found no significant difference between the survivorship of unmarked control mosquitoes and that of marked specimens, or between the observed survival rate of marked specimens compared to other findings (Reisen et al. 1980; Kempala 1981; Chiang et al. 1991; Takken et al. 1998; Watson et al. 2000; Johnson et al. 2012; Liu et al. 2012; Marini et al. 2019).

Only one study of the effects of dust application on adult mosquitoes found that the survival rate of marked mosquitoes was dependent on the method and colour of the dust application, as well as the sex of the individual (Dickens and Brant 2014). However, this study was not carried out under field conditions and its applicability to dispersal studies may be limited. Additionally, these findings stand in contrast to others: In a field-based MRR study in West Africa, investigators found no effect on survival attributed to dust colour (Epopa et al. 2017). Another study on the effects of 7 types of fluorescent powders by 4 different manufacturers on female Ae. aegypti survival found no significant differences in survival among unmarked and marked females (Rojas-Araya et al. 2019). This study did find that marked females were less likely to be captured by traps than unmarked mosquitoes, except for females who were marked with DayGlo formaldehyde- 
free powder from the ECO series. Verhulst et al. (2013) found no effect of marking by fluorescent powder on the survival of An. gambiae that were treated fewer than 3 days following emergence, though survival was significantly reduced in cohorts treated more than 5 days after emergence. A later study found that fluorescent powders had no effect on females across different age cohorts, which included females up to 13 days after emergence (Rojas-Araya et al. 2020). These authors also reported that marking dust had low impact on blood-feeding behaviour and tethered flight speed. The remainder of the literature largely suggests that fluorescent dust application is a benign marking procedure that has little effect specifically on mosquito survival or behaviour if applied appropriately (Reisen et al. 1979; Curtis and Rawlings 1980; Nelson and Milby 1980; Beier et al. 1982; Muir and Kay 1998; Hagler and Jackson 2001; Ageep et al. 2014; Guerra et al. 2014a).

Dust application effects on sensory organ functioning are not well-studied in mosquitoes. One study by Verhulst et al. (2013) supports the use of fluorescent dusts in mosquito MRR studies, finding that their host-seeking response to human or cow odour was not affected by its application.

Few studies have investigated or remarked on the frequency of transfer of marking dusts to other mosquitoes or surroundings. Meek et al. (1988) observed no pigment transfer when marked adult mosquitoes when confined in cages with unmarked adults, nor when the former were mated with the latter. Subsequent studies (Fryer and Meek 1989) found that between 0.1 and $3 \%$ of unmarked adults became marked after 24 hours of confinement with heavily dusted adults. However, these authors also found that mosquitoes sheltering within vegetation where dust had been applied could be marked by pigment transfer up to 4 days after dust application. A study by Nelson et al. (1978) observed some recaptured $C x$. tarsalis females showed dust markings only on their external genitalia, suggesting the possibility of dust transfer during mating. However, a study by Rojas-Araya et al. (2019) found that recaptured mosquitoes never had only a single marking on the body, but had many. They also never found specimens marked with two different coloured dusts, suggesting that no transfer occurred between individuals in a semi-field environment. No other MRR studies on mosquitoes expressed suspicions about dust transfer, and it appears to be rare enough to quell concerns about contamination. 


\section{Release procedure}

While logistically challenging to optimize, the number of release sites, as well as the timing of release events may influence the recapture rates and dispersal capacity of marked mosquitoes. The use of both single release sites (70\%) and single release events (70\%) are most commonly reported across past mosquito MRR experiments in comparison to multiple sites (30\%) or events (24\%) (Guerra et al. 2014). Release of marked mosquitoes from multiple sites or in multiple events is advantageous in accounting for the effects of wind direction and other climatic factors such as precipitation. However, these approaches to $\mathrm{MRR}$ require a significantly greater amount of labour and resources, and are logistically difficult to perform, especially on a larger geographic scale.

Past mark-release-recapture experiments and other observations suggest that the first 1 to 4 days after emergence are the most important for active dispersal behaviour (Nayar 1985; Watson et al. 2000), likely because survivorship declines quickly after this period (Y. Li et al. 2014). The time of day in which marked mosquitoes are released has not been shown to affect their recapture rate or mean dispersal distance (Reisen et al. 2003). Mosquitoes have been observed to fly upwind when air currents are less than the flight speed of mosquitoes (Snow 1976). This speed is often reported as approximately $1 \mathrm{~m} / \mathrm{s}$, but the flight capabilities reported vary by species (Bailey et al. 1965; Bidlingmayer and Evans 1987). In $C x$. tarsalis, some observations found them to fly primarily with the wind at velocities above $2.7 \mathrm{~m} / \mathrm{s}$ (Bailey et al. 1965).

If active dispersal capabilities are the focus of the MRR experiment, it is generally wise to release individuals in conditions where the wind speed is greater than $1 \mathrm{~m} / \mathrm{s}$, otherwise upwind flying is less likely to be successful (Bidlingmayer and Evans 1987). Findings by Lacroix et al. (2009) found that the majority (73\%) of mosquitoes in their mark-release-recapture experiments (all Aedes albopictus (Stegomyia albopicta) (Skuse 1894)) were recaptured upwind in low wind velocity conditions. They suggested that the odor plumes of potential hosts had attracted them, which Russell et al. (2005) have also suggested to explain upwind movement of Ae. aegypti in their studies. Others have cautioned that wind of any velocity will result in fewer trap catches, with earlier findings that only extremely low wind speeds of less than $0.2 \mathrm{~m} / \mathrm{s}$ had no effect on catch rates 
(Bidlingmayer et al. 1985; Bidlingmayer et al. 1995). In a study where mosquitoes were released within an orchard, wind speeds averaging between 7 and $11 \mathrm{~m} / \mathrm{s}$ prevented mosquitoes from dispersing from the orchard. It was noted that most females only dispersed outside the orchard when wind speeds fell below $4.5 \mathrm{~m} / \mathrm{s}$, and even so, were seen in greater numbers at leeward traps (Reisen et al. 2003).

\section{Recapture procedure}

Guerra et al. (2014) noted that recapture techniques vary across the literature, with aspiration $(\sim 30 \%)$, human or animal bait $(\sim 30 \%)$ and $\mathrm{CO}_{2}$ light traps $(\sim 20 \%)$ representing most methods used, while only $5 \%$ of studies used basic light traps. Others have pointed out the limitations of biased methods of mosquito collection, such as light traps baited with $\mathrm{CO}_{2}$, which reflect only the distribution of blood-seeking mosquitoes (Chuang et al. 2011). Baited traps may also underestimate mosquito activity at host-rich sites, as the presence of hosts has been shown to override the attraction of $\mathrm{CO}_{2}$-baited traps in $C x$. tarsalis (Thiemann et al. 2011). Burkett-Cadena et al. (2013) recommended the use of nonattractive capture methods such as aspirating mosquitoes from resting shelters, as to not disrupt the natural distribution by attracting mosquitoes from elsewhere. While inexpensive, the labour required to aspirate specimens renders it an unfeasible method for experiments measuring dispersal over a large range. In this circumstance, non-baited light traps offer a compromise between convenience and effectiveness.

While rare in MRR studies, the New Jersey light trap (NJLT, see Ch. 3.2.2) is the standard method of collection used by most mosquito control programs (including the City of Winnipeg Insect Control Branch) to measure mosquito activity and species presence (Reinert 1989). Operation of NJLTs allows for the same recapture effort to be applied from one trap night as that from one human aspirator for a 15-min period (Mulhern 1934). While each NJLT unit is priced at 400 dollars US (BioQuip Products 2019), those conducting MRR experiments may offset this cost by collaborating with local mosquito control authorities who often use them as well.

However, not all species can be reliably collected by light traps, given that not all species are phototropic or active at night, when the contrast of the trap light to its surroundings are greatest (Reinert 1989). Light traps vary in their ability to attract and 
collect many of the species found in Manitoba (Wood et al. 1979). According to Reinert (1989), species not reliably collected by lights traps are Aedes (Ochlerotatus) canadensis (Theobald, 1901), Aedes (Ochlerotatus) stimulans (Walker, 1948), Aedes (Ochlerotatus) triseriatus (Say, 1823), Aedes (Ochlerotatus) excrucians (Walker, 1856), Cx. restuans, Culex territans Walker, 1856, Anopheles punctipennis (Say, 1823) and Culiseta melanura (Coquillett, 1902). Trap counts in NJLTs have also been found to be relatively lower in urban areas where there are competing light sources (Milby and Reeves 1989). For these reasons, many mosquito control agencies have incorporated other sampling methods into their surveillance programs, such as $\mathrm{CO}_{2}$ baited traps (Reisen et al. 2002). Additionally, as light disrupts normal insect behaviour, light traps could be said to create a more artificial situation than traps with host-like attractants (Silver 2008: 847). Nevertheless, most species are visually attracted to conspicuous objects and orientate toward traps from a distance of 15 to $20 \mathrm{~m}$, such that these traps may still attract these species during the day (Bidlingmayer and Hem 1980). Despite varying levels of attractiveness, NJLTs are still one of the most effective and economical methods to monitor the populations of adult mosquitoes.

Previous mosquito MRR studies have indicated that most marked individuals are recaptured in the 3 to 4 days following release (Reisen et al. 1992; Reisen and Lothrop 1995). The duration of recapture effort is at the discretion of the investigators and resources available, though longer recapture periods are advisable if survivorship or maximum dispersal distance is of interest.

The placement of individual traps has also been shown to significantly affect trap collections and the outcomes of MRR experiments. Sources of variation may stem from only a few meters of difference in trap placement, such as a trap's proximity to larval habitat, resting areas, livestock animals or artificial light sources, as well as its degree of wind exposure (Barr et al. 1963). In the case of NJLTs, their use is limited to areas with access to electricity and space for installation. Variation in light trap collections can also be influenced by the intensity of moonlight (Barr et al. 1960), as well as nightly temperature and humidity (Service 1976), though individual species may be affected to different degrees. 
As a final remark on considerations for recapture, the spatial configuration of traps used must be appropriate for the research questions being addressed. Underestimates of dispersal may arise if the study area is too small to capture the full dispersal range of the mosquitoes studied. More traps deployed near the release site may result in more recaptures, but may compromise dispersal distance (Guerra et al. 2014a). A checklist of essential information for MRR experiments also recommends that the positioning of traps relative to the release sites be considered. However, not enough information regarding the influence of symmetrical, asymmetrical or circular arrangements of traps is currently available to draw reliable conclusions (Guerra et al. 2014a).

\section{Past work}

It is inadvisable to extrapolate the results of a single MRR study conducted in one location to inform control program activities in another as different populations of the same species may exhibit different dispersal patterns depending on the surrounding environment (Benedict et al. 2018). For this reason, only MRR studies that concern themselves with species of interest (see 2.2) or take place in regions with comparable climate and topography as the study region in Manitoba, Canada will be reviewed here.

Vectors mosquito species which are more common in MRR studies, such as Ae. aegypti and a species of interest, $C x$. tarsalis, report average female recapture rates of $16 \%$ and 5.1\%, respectively (Guerra et al. 2014b). Of 113 MRR experiments across 15 studies dealing with $C x$. tarsalis, all were carried out in California between 1961 and 2002 (Guerra et al. 2014b). Of these studies, most did not seek to measure dispersal distance and so did not report it. A review of 10 observations of $C x$. tarsalis dispersal calculated an average maximum flight distance of $13.7 \mathrm{~km}$ (Verdonschot and Besse-Lototskaya 2014).

There is comparatively less data from MRR studies for the other species of interest in this study. Only 5 MRR studies which address Ae. vexans were found, and the average recapture rates in these experiments were relatively low at $0.41 \%$ (Table 1). Two other studies on the dispersal behaviour of Ae. vexans did not yield quantitative information (Clarke 1937; Clarke and Wray 1967). In their meta-analysis, Guerra et al. (2014b) found only 3 experiments across 2 mosquito MRR studies which released Ae. dorsalis and these reported an average female recapture rate of $0.56 \%$. Two studies on Ae. dorsalis yielded 
an average maximum distance of nearly $7 \mathrm{~km}$ (Verdonschot and Besse-Lototskaya 2014). This is perhaps due to the large area required to measure the dispersal of mosquitoes classified as strong fliers, such that there is lower trap density at greater distances from the release site(s), making recaptures rarer. These studies are most limited by their recapture rates, which creates problems when drawing conclusions from statistical analysis. Hocking (1953) acknowledged the difficulty of measuring dispersal with data from MRR studies, as "good results" are easier to obtain using insects with limited flight ranges and landscapes where the terrain is not difficult to traverse. No MRR studies observing the dispersal of $C x$. restuans, Cs. inornata or Cq. perturbans were found in a literature search.

Table 1. Review of mark-release-recapture studies of Aedes vexans. As certain values are not reported in these studies, where possible, they have been inferred from data or figures provided in each publication.

\begin{tabular}{|c|c|c|c|c|c|c|}
\hline \multirow{2}{*}{ Reference } & \multirow{2}{*}{$\begin{array}{l}\text { No. marked } \\
\text { and released }\end{array}$} & \multirow{2}{*}{$\begin{array}{c}\% \\
\text { recaptured }\end{array}$} & \multicolumn{2}{|c|}{$\begin{array}{c}\text { Max. flight } \\
\text { distance }(\mathbf{k m})\end{array}$} & \multicolumn{2}{|c|}{$\begin{array}{c}\text { Mean flight } \\
\text { distance (km) }\end{array}$} \\
\hline & & & $\mathbf{F} q$ & $\mathbf{M} \widehat{\partial}$ & $\mathbf{F} q$ & $\mathbf{M} \widehat{\partial}$ \\
\hline Ba et al. 2005 & 85,500 & 0.18 & 0.62 & N/A & \multicolumn{2}{|c|}{0.35} \\
\hline Bogojević et al. 2011 & $50,000^{\mathrm{a}}$ & 0.04 & \multicolumn{2}{|c|}{7.7} & \multicolumn{2}{|c|}{4.3} \\
\hline Brust 1980 (1976 cohort) & $1,000,000^{\mathrm{b}}$ & 0.007 & \multicolumn{2}{|c|}{11.0} & & \\
\hline Brust 1980 (1977 cohort) & $1,000,000^{\mathrm{b}}$ & 0.02 & \multicolumn{2}{|c|}{8.0} & 2.0 & N/A \\
\hline Jensen and Washino 1994 & 3,231 & 2.0 & \multicolumn{2}{|c|}{ N/A } & 0.11 & N/A \\
\hline Stage et al. 1937 & $>100,000^{b}$ & $<0.21$ & \multicolumn{2}{|c|}{8.0} & \multicolumn{2}{|c|}{3.2} \\
\hline
\end{tabular}

${ }^{a}$ Includes counts from Aedes (Ochlerotatus) sticticus and Aedes (Ochlerotatus) caspius

${ }^{\mathrm{b}}$ Includes counts from Aedes (Ochlerotatus) sticticus (formerly Aedes aldrichi)

The number of published MRR studies conducted in Canada is limited to only two experiments in Manitoba by Brust (1980). The study investigated the dispersal behaviour of Aedes (Ochlerotatus) sticticus (Meigen, 1838) and Ae. vexans from agricultural floodplains outside the city of Winnipeg. A total of over 2.25 million mosquitoes were released during experiments in 1976 and 1977, in which mosquitoes were reared from field-collected larvae in screened enclosures, dusted with DayGlo fluorescent powder and recaptured in NJLTs. The study also collected adults by sweep netting within $100 \mathrm{~m}$ of the release to determine if marked adults had a 'refractory period' in which they remained at the release site. Brust (1980) demonstrated that many adults remained at the release site for a few days following release, especially if temperatures were lower. Aedes vexans travelled primarily with the wind and flew to the furthest trap $(8 \mathrm{~km})$ within 3 to 5 nights after 
release, with some adults travelling $3 \mathrm{~km}$ in their first night. However, he noted that prevailing winds in Winnipeg during June and July originate from the south, and that the influence of the lit urban horizon could not be separated from that of the wind, though there is no evidence to suggest that the lit horizon influences dispersal. Mean nightly temperatures were correlated with the total number of mosquitoes collected in traps. Most traps were located on farms or rural residential locations, some of which hosted livestock, a factor which may have attracted the high percentage of female mosquitoes trapped at these traps.

Concerning factors that affect dispersal in MRR experiments, Verdonschot and BesseLototskaya (2014) noted that in most studies, the environmental conditions under which the experiments were performed were not adequately reported and their potential influence on dispersal behaviour was not discussed. However, there are data suggesting that landscape features, such as surface water, and vegetation height and density may act as corridors wherein mosquitoes can orient with the silhouette of continuous vegetation such as trees (Bidlingmayer 1975; Bidlingmayer and Hem 1981; Schäfer et al. 1997), especially if these are near a body of water where the moisture profile is relatively high. Presence of standing water near these areas suggests available breeding sites, which may further affect dispersal patterns (Reiter et al. 1995). Additionally, shaded or windprotected areas offer an attractive habitat for most mosquitoes when compared with open areas for their protection against heat and desiccation (Schäfer et al. 2004). Mosquitoes may take advantage of the presence of potential blood meal hosts (e.g., birds, mammals, etc.), which also frequent these areas (Cederlund and Okarma 1988; Tufto et al. 1996).

Also important to note is the importance of mean nightly temperatures, asserting that dispersal flights are unlikely to occur at nightly temperatures lower than $15^{\circ} \mathrm{C}$ under Manitoba conditions (Brust 1980). Other meteorological factors may also affect the success of dispersal but are likely contingent on many other ecological variables, including landscape features and mosquito species.

\section{Data analysis}

Typically, numbers of mosquito recaptures decrease with increasing distance from the release site. While this is usually attributed to the extent of typical distances flown 
mosquitoes, it is also influenced by the decrease in sampling effort per unit area as the size of the trapping area increases outwards radially (Silver 2008). A meta-analysis by Guerra et al. (2014a) noted that very few studies compensated for the decrease in recapture success by reduction in sampling effort as a function of distance from the release point. This may be compensated for by assigning each trap to an "annulus", or circular area around the release site which excludes smaller annuli. The area of a circular trapping annulus $(A)$ is denoted as $A=\pi\left(R^{2}-r^{2}\right)$, where $R$ is the distance from the outer edge of the annulus, and where $r$ is the distance from the inner edge of the annulus. For example, suppose that the $1^{\text {st }}$ annulus in a trapping area has an outer edge that is $1 \mathrm{~km}$ radius from the release site, and that the outer edge of all subsequent annuli is $1 \mathrm{~km}$ further than the previous. This $1^{\text {st }}$ annulus will cover a smaller area than a trap in the second annulus $\left(3.14\right.$ and $9.42 \mathrm{~km}^{2}$, respectively). This means that if there are 4 traps in this first annulus, 12 traps would be required within the second annulus to match the sampling effort of the first (1.27 traps per $\mathrm{km}^{2}$ ). If there are more than 6 annuli, at least 144 traps would be required in total.

This creates logistical problems for MRR studies where the species of interest may disperse over great distances. Therefore, fewer traps are used at outer annuli and a correction factor (CF) to compensate for the disparity in sampling effort for each annulus is calculated and applied (Lillie et al. 1981; Brenner et al. 1984; Lillie et al. 1985; Morris et al. 1991). The number of marked mosquitoes present in each trap is then divided by number of traps in the same annulus. These values are then multiplied by their correction factor to derive the estimated number of specimens which would have been recaptured if sampling effort in each annulus was equal the annulus with the greatest sampling effort (see 3.2.5). Any distance can be used as for spacing, so long as there is at least one recapture site in each annulus (Morris et al. 1991). However, the assumptions made by this rule are not well-understood or discussed in the literature.

In analyses of mark-release-recapture data of mosquitoes, dispersal is typically measured by mean distance travelled (MDT) and can be calculated with compensation for unequal trap densities at greater distances (See 3.2.5 and Morris et al. 1991). Otherwise, if the mean distance travelled is calculated using the raw observations from recaptured mosquitoes, then the average dispersal distance will be underestimated due to the 
overrepresentation of traps nearer to the release site. Even with the corrected data, Bogojević et al. (2011) noted that researchers should be prudent in using MDT values to inform policy decisions such as buffer zone width, because MDT likely still underestimates these values by assuming a linear flight distance from release to recapture, whereas in reality mosquitoes may follow more indirect routes. Additionally, dispersal in insects is often leptokurtic, such that observations are clustered around a true mean which is very sensitive to a few insects dispersing much further than most. Therefore, it is important to distinguish between mean (MDT), median and maximum flight range when reporting results (Benedict et al. 2018). Verdonschot and Besse-Lototskaya's (2014) meta-analysis of mosquito dispersal studies reported no clear relationship between maximum and average flight distance. However, this meta-analysis was not species-specific, nor centred on MRR experiments. The authors asserted that flight mill and transect data show large differences when compared with dispersal data obtained from MRR experiments.

While maximum flight range must be reported using raw observations, the median can be calculated using a regression equation of the cumulative number of recaptures against log-transformed distance. Solving for $y$ at the point where the cumulative proportion of recaptures is $50 \%$ gives a flight range for half of the recaptured mosquitoes, which is mathematically the median flight distance (Morris et al. 1991). Other useful parameters can be obtained from this method as well, such as the flight range for $90 \%$ or any other proportion of the adult population, which may be more reliable than a maximum flight range based on only a few individuals. Other ways of representing dispersal data include using the development of models, typically made with the inclusion of random effects such as marking method, time elapsed prior to recapture, recapture location (Medeiros et al. 2017), and mosquito condition (Juarez et al. 2020). For example, the probability of detecting a marked mosquito at a given distance has been iterated with different models in recent publications, though this research is largely nascent (Hamer et al. 2014; Medeiros et al. 2017; Juarez et al. 2020).

\section{4 - Spatial analysis in the context of mosquito control}

Mosquito-borne diseases (MBD) were recognized as a threat with a spatial component long before mosquitoes were identified as their vector; prior to the discovery 
of its mechanism, many attributed the transmission of malaria (Italian for "bad air") to "noxious emanations of swamps" (Reiter 2000; Hempelmann and Krafts 2013). Since then, recognition of the geographic limitation of many disease outbreaks, coupled with principles of landscape ecology have led to the emergence of the field of "spatial epidemiology". The primary maxim of landscape ecology asserts that the spatial heterogeneity of populations arises from their interactions with physical and/or biological structures across the landscape that surrounds them (Ostfeld et al. 2005). More specifically, landscape ecologists are interested in the influence of the composition and configuration (position) of landscape features on the distribution of organisms across a landscape. The distribution pattern that arises from these influences is statistically known as "dispersion", not to be confused with dispersal, which is the distribution change that results from movement (Reisen 2010). The framework of landscape ecology is medicalized by spatial epidemiologists - or "landscape epidemiologists" (Reisen 2010) - who use information on human demographics and behaviour alongside physical factors in the environment to explain the spatial patterning of infections.

Within studies of MBD epidemiology, a wide variety of spatial data on multiple scales are employed to predict the risk of transmission and outbreak. Often selected as spatial variables are different measures of land cover, land use, climate, soil type, elevation, density of human (or other host) population, and the quantity and type of vegetation (Kitron 1998). These characteristics describe the potential habitats of either the hosts, or the mosquitoes, who prefer specific environments to breed, rest and nectar-feed. The objective in extracting these variables is to quantify their relationships to the pathogen (or its vector) in the context of the larger landscape. The goal is to delimit those relationships on maps that can give a visual estimate of the spatial variance in risk of outbreak within their borders. Risk maps are valuable components of surveillance systems as they can practically inform authorities tasked with managing the risk of transmission (Kitron 2000). This approach is also useful to authorities where mosquito control is done predominantly for aesthetic purposes, as the distribution of mosquitoes across a heterogenous landscape such as a city can also be inferred this way. Map projections of the distribution of adult mosquitoes based on spatial relationships allow for more efficient allocation of resources by the targeting of areas that would benefit most from control efforts. 
However, the task of defining the landscape and delimiting its features on maps, involving lengthy (or exhaustive) ground surveys of the study area, was excessively laborious until only relatively recently. Early approaches to investigating the effects of landscape structure on the distribution of a population typically used aggregated measures, based on the frequency that a geographic feature was encountered within a plot or sampling unit (Beier et al. 1982). This approach not only limited the scale at which patterns can be detected, but it also neglected to account for the underlying spatial correlation between observations across geographic space. This issue known as spatial "autocorrelation" or "dependency", is common in ecological phenomena and upholds that geographic features that are closer together are more related than those that are further apart (Tobler 1970). It is corollary that observations made across a landscape would not be independent of one another, violating the assumption of independence present in most parametric statistical procedures. For this reason, ecological data should always be investigated for spatial autocorrelation, as it may introduce bias, overestimate the significance of a variable's influence and produce false positives in the results (Legendre 1993; Diniz-Filho et al. 2003; Ostfeld et al. 2005).

But at the turn of the $21^{\text {st }}$ century, advances in Geographic Information Systems (GIS) and remote sensing (RS) technologies, coupled with the development of spatial statistics, provided new means of approaching the analysis of spatial data (Kitron 1998; Reisen 2010). The widespread availability of Global Positioning System (GPS) units allowed for the exact location of each sampling point to be measured inexpensively virtually anywhere on earth. With this, observations could be spatially linked to a specific GPS location within a computer-based GIS application. These applications are everincreasing in functionality and provide many tools that allow users to manipulate and analyze spatial data, and to produce maps to visualize results.

The usefulness of GIS tools was further magnified with the integration of data from instruments aboard an increasing number of earth-orbiting remote sensing (RS) satellites. Remote sensing is the monitoring of environmental conditions using sensors that measure the energy emitted or reflected from a unit area or "pixel" on the surface of the earth (Washino and Wood 1994; Ostfeld et al. 2005). These measure the intensity of outgoing 
radiation within subsections ("bands") of the electromagnetic spectrum, typically in the visible or infrared range (Kitron 1998; Reisen 2010), though other frequencies, such as microwaves, are sometimes used as well (Chuang, Henebry, et al. 2012). While these bands do not directly measure any habitat characteristics, they are sensitive to variations in two factors essential to mosquito survival: vegetation and water (Washino and Wood 1994; Richards et al. 2006; Reisen 2010). Variations across multiple bands can be integrated to extract information about land cover that can be used to identify mosquito habitats. As RS satellites orbit the earth continuously and can measure the same surface area at regular intervals, they are invaluable tools for monitoring the effects of landscape change over time.

More recently, many have developed statistical procedures that account for spatial autocorrelation in the data and used them to determine the number and type of influential variables that drive vector population distribution. These often employ regression methods to model the relationship between the dependent variable, such as the presence or activity level of vectors, and the explanatory variables in the environment (Sallam, Fizer, et al. 2017; Uusitalo et al. 2019). To represent the results on risk maps, the most important predictors are used to model mosquito activity ("risk") in areas not sampled by traps (Kitron 2000; Piedrahita et al. 2020). For epidemiologists and control operations, mosquito distribution inferred by these risk-forecasting models are functionally equivalent to maps produced by species distribution models that have been developed for conservation purposes. These models are correlational rather than mechanistic and are developed based upon the supposed ecological niche of the species in focus (Qiao et al. 2015).

Production of risk maps vary in their methods, but generally follow a three-step approach, adapted from Ostfeld et al. (2005). First, explanatory variables are selected and extracted within a set of zones around mosquito sample locations. Statistical analysis is then performed to find the set of variables that together best predict species distribution, while reducing redundancy or collinearity between these predictors. Finally, the variables selected from this analysis can be used to forecast the distribution of mosquitoes on the landscape based on the information about these variables that is present at each pixel. Risk maps produced by this approach can guide mosquito control operators in targeting areas 
with high mosquito populations. Even the optimal timing of control actions could be informed by the projections of sophisticated multi-system models if meteorological data were to be included in the model in real-time.

In the final three sections of this chapter, I will discuss common procedures for modeling following the three-step approach described above. This review will be tailored to give attention primarily to methods that are consistent with my goal of producing a static mosquito activity risk map for the Winnipeg area based on landscape-level variables (see Ch. 4).

\section{Selection and extraction of spatial variables}

Static mosquito risk maps are usually based on of one the following three empirical datasets: human disease cases, non-human reservoirs, or known mosquito surveillance records (Ostfeld et al. 2005). As the former two are disease-specific and not applicable to risk mapping for mosquito control for nuisance abatement, I will narrow our discussion to only methods that use the known distribution of mosquitoes themselves (specifically adult females) as the responding spatial entity. Most distribution models are based on data on mosquito presence or activity, though sampling methods vary widely (Sallam, Fizer, et al. 2017). While activity data (often referred to as "abundance") are time-consuming to obtain, they are preferable to presence/absence or presence-only data in circumstances where there is a relatively high species population density throughout the study areas (no "absence"), such as the case with mosquitoes. It also allows for the identification of population hotspots and distinction between more densely or sparsely populated habitats, as evidenced by varying mosquito counts at different traps. Counts from the same trap are often aggregated (Burkett-Cadena et al. 2013) or averaged (Diuk-Wasser et al. 2006; Field et al. 2019) to summarize variation in trap collections during the sampling period.

Before discussing the extraction of variables that best predict this distribution, it is important to note that landscape-based modeling is not the only approach to forecasting mosquito activity in unsampled areas. Another approach used in pest management, known as interpolation, depends only on the assumption of spatial autocorrelation among the trap counts (Sciarretta and Trematerra 2014). Kriging is an interpolation technique sometimes used in pest management in which the value of a variable (such as the probability of 
mosquito presence) at a point in space is predicted by a weighted average of the point values nearest to it (Brenner et al. 1998). This technique has been used produce risk maps that show potential vector population hotspots separated by areas of incrementally lesser habitat density (Ryan et al. 2004; Diarra et al. 2018; Bunn et al. 2019).

However, the accuracy of kriged maps depends on how well the sample data represents the overall spatial heterogeneity within the study area. Interpolation is not a favorable approach to map distribution over landscapes with high spatial heterogeneity, where traps might not be spatially autocorrelated if they are too far from one another (DiukWasser et al. 2006). Traps used as part of mosquito surveillance systems in cities tend to be placed in non-uniform patterns dictated by access and convenience with wide expanses of variable terrain between them (Reisen and Lothrop 1999). Further, mosquitoes are widely known to be driven, in a manner that is species-specific, towards certain environments that allow for optimal larval development, feeding, and resting (Porphyre et al. 2005; Ferraguti et al. 2016). Therefore, the development of landscape-based model is a more appropriate approach to modeling the distribution of mosquitoes in a large, spatially heterogenous area such as an urban area.

Meteorological or climatological data (i.e., temperature, precipitation) are often used as predictors alongside landscape-based variables. These factors dictate the distribution of vegetation and standing water, and therefore, the extent of suitable habitat (Richards et al. 2006; Reisen 2010). Meteorological data are useful in predicting when adult populations will spike, as larval development times, and therefore generation times, are shortened at higher temperatures (Tun-Lin et al. 2000). When determining the potential range of a species, climatological variables are essential to estimate mosquito distribution on a country or continent-wide scale (Benedict et al. 2007). However, at smaller scales individuals are responding to a wide range of microclimates, or conversely, areas with climatic conditions that differ from their surroundings (Murdock et al. 2017; Wong and Jim 2017). Microclimate is mediated by local vegetation structure and topography that more precisely outline their habitats within a heterogenous landscape (Suggitt et al. 2010). For instance, after a rainfall event, the amount of standing water made available for oviposition and larval development at any given location is dependent on antecedent 
wetness, surface permeability, and rates of evapotranspiration present (Shaman and Day 2005).

While climatic and meteorological variables affect the relative seasonal or daily abundance of mosquitoes (Kovats et al. 2001), it has been emphasized that the principal determinant of their presence and relative abundance is more reliably predicted by local ecology, land cover and use (Reiter 2001; Zittra et al. 2017). Reiter (2001) warned that models hinged on climate-based variables were inappropriate to predict future spread of MBD given the more severe impacts of human activities on local ecology. As habitat characteristics usually describe relatively static or permanent features such as vegetation and land cover, these are more reliable predictors of mosquito distribution in general. Additionally, climatic measurements are typically coarse in their resolution, where one or two data points are commonly used to represent the conditions across a relatively large region (Demets et al. 2020). The distribution of water within an urban area, where there is a high density of surfaces that are impervious to water, are not adequately represented by these measurements. Further, the resolution of readily available climatic data is larger than the lifetime dispersal range of most mosquitoes, no more than a few dozen kilometres at most (Hamer et al. 2014; Verdonschot and Besse-Lototskaya 2014). It is for these reasons, that environmental variables pertaining to weather and climate are excluded from this review.

Among landscape-level predictors, variables that describe land cover and/or land use (LULC) are most common, though some studies include elevation and slope if there is sufficient landscape heterogeneity to suspect influence (Chuang and Wimberly 2012; Sallam, Fizer, et al. 2017). Challenges related to the precise definitions of LULC variables arise when attempting to compare spatial analyses of mosquitoes, as there are no established standards for the extraction of these variables (Sallam, Fizer, et al. 2017). I refer to this definition of land cover and land use from Izzah et al. 2016: Land "cover" refers to the physical features on the earth's surface, such as vegetation, bodies of water and bare rock. Land "use" considers the socio-economic functions of these areas such as whether they are primarily used for agricultural, residential, or commercial purposes. Land use and cover are used interchangeably in this thesis, as the relationship between physical 
features and human usage are inseparable within the urban areas in focus in this review. Additionally, while remote sensing techniques do not directly measure land use or land cover, they allow for the measurement of spectral information which can be used to infer these land use and cover (Natural Resources Canada 2015). In many mosquito distribution modelling studies, LULC information in thematic maps are obtained from national databases, where they are available as pre-classified data products for general usage (Chuang, Hockett, et al. 2012; Healy et al. 2013; Zittra et al. 2017). Where pre-existing LULC data are unavailable or otherwise unsatisfactory for its intended purposes, a landscape classification can be performed in a variety of ways using GIS software.

Another maxim of landscape ecology must be introduced here: results and conclusions from spatial analyses are "profoundly" influenced by the scale at which the landscape is characterized (Turner 1989; vonHedemann et al. 2015) - this is the basis of the modifiable area unit problem (Dark and Bram 2007). The type of landscape classification selected, either pixel or object-based, depends on the scale of the landscape features or "objects" of interest, and on the resolution of the RM imagery used (Blaschke 2010).

Pixel-based classifications are sometimes used to characterize landscapes for mosquito distribution studies (Vanwambeke et al. 2007; Landau and van Leeuwen 2012; Bunn et al. 2019; Field et al. 2019; Lorenz et al. 2020). These can be performed with both "unsupervised" or "supervised" procedures. In an unsupervised procedure, a clustering algorithm assigns individual pixels in a remotely sensed image into different classes (to be identified later by the user) based on their "colour" or spectral values (Puletti et al. 2014). To run a supervised classification, a user first needs to delineate training samples, or representative examples of the LULC classes one intends to differentiate. A classifier algorithm then assigns individual pixels into the land cover classes specified using spectral information obtained from the pixels in the training samples. Examples of common classifier algorithms used are the iterative self-organizing (ISO) cluster, maximum likelihood, Random Forest (RF) and support vector machine (Vanwambeke et al. 2007; M. Li et al. 2014; Thanh Noi and Kappas 2017; Lorenz et al. 2020) 
Pixel-based classifications perform best when the landscape features (or "objects") of interest and the resolution (pixel-size) of the RM image are similarly-sized, or when the latter is larger (Hay et al. 2001; Blaschke et al. 2004). For instance, if the presence of large forest stands or agricultural fields is of interest, a resolution of $250 \mathrm{~m}$ or greater would be sufficient to obtain accurate information from a pixel-based classification. However, urban areas are greatly heterogenous at comparatively much finer scale (Stefanov et al. 2001; Herold et al. 2003; Maktav et al. 2005). The coarse resolution of many RM satellites hampers their ability to distinguish between LULC variables in urban areas, as they cannot distinguish spatial details beyond "built land" or "urban" (Brown et al. 2008). This occurs because the small size of landscape features may lead to significant subpixel mixing (Ridd 1995; Foody 2000), and subsequent misclassification of land cover. Therefore, it is recommended that those wanting to extract LULC data at a scale relevant to urban areas should use spatial information recorded at a resolution of $10 \mathrm{~m}$ per pixel or less, in alignment with the scale of larger urban features such as buildings, roads and parks (Anderson et al. 1976; Herold et al. 2003). Considering the scale of a mosquito's individual experience in an urban area, which range from microhabitats such as tree holes and discarded man-made containers, to backyards, and to larger, more conspicuous biomes such as wetlands (Brown et al. 2008; Shaw et al. 2013), a $10 \mathrm{~m}$ per pixel guideline is a reasonable guideline for the characterization of relevant habitat.

At a spatial resolution of 10 metres, certain urban features that are relevant to mosquitoes, such as residential, vegetated, and agricultural areas, will be represented by more than one pixel. When the available resolution of RS imagery is finer than the objects of interest, an object-based landscape classification should be used rather than a pixelbased classification (Blaschke et al. 2014). Using training samples, this approach groups pixels together into digital "objects" based on their color (or spectral), size, shape, texture, and context from pixels in their surroundings (Blaschke et al. 2014). In doing so, both contextual and spectral information are considered by the classification algorithm, leading to a more accurate LULC classification in comparison to a pixel-based classification of the same imagery (Weih and Riggan 2010). 
The landscape variables of interest to mosquito distribution studies are commonly focused on vegetation and hydrology (Lothrop and Reisen 1999), as well as the extent of urban or "built-up" area. Hydrological data often intends to approximate soil moisture or the potential for the accumulation of standing water, which is essential for the reproductive cycle of all mosquitoes. Measures used to represent a region's hydrological properties include topographical wetness index (Conley et al. 2014), ponding frequency, and proximity to wetlands, (Chuang, Hockett, et al. 2012), drainage infrastructure (Deichmeister and Telang 2011), or permanent bodies of water (Diuk-Wasser et al. 2006; Gleiser and Zalazar 2010; Landau and van Leeuwen 2012). Remotely sensed vegetation indices, such as the normalized difference vegetation index (NDVI), are commonly used to represent the variation in primary production across the landscape (Kitron and Kazmierczak 1997; Gleiser and Zalazar 2010; Chuang, Henebry, et al. 2012; Conley et al. 2014; Ferraguti et al. 2016). Land cover classes such as cultivated, tree canopy or grassy areas are often extracted for use as variables in regression models (Deichmeister and Telang 2011; Burkett-Cadena et al. 2013), as these may act as resting sites for mosquitoes and their hosts, especially in urban areas (Brown et al. 2008; Kwon et al. 2015; Crocker et al. 2017).

Built land, building cover and impervious surfaces are also frequently included (Deichmeister and Telang 2011), as these collect water and may represent up to $93 \%$ of larval habitats in urban areas (Keating et al. 2003). The presence and characteristics (structure, materials, age, etc.) of urban dwellings also signify the presence of human hosts, which is particularly important in studies of anthropophilic mosquitoes, or those where the epidemiology of an MBD outbreak is concerned (Ostfeld et al. 2005; Kalluri et al. 2007). Other urban-specific features vary in their influence on mosquito abundance. Reiter and others (1995) found that buildings did not impede on the flight of Ae. aegypti, and large highways have been shown to act as a barrier to dispersal for these mosquitoes (Hemme et al. 2010). Mosquitoes may find that the lack of shade and emanating heat from the pavement create conditions too harsh to traverse (Tun-Lin et al. 1995).

Population density and other socioeconomic metrics such as income are also sometimes used as predictive variables, as they can be represented as a mosaic across an 
urban area (Conley et al. 2014; Ferraguti et al. 2016; Zittra et al. 2017). Urban areas with larger populations living in poverty have been observed to have higher mosquito activity, as water stored in open containers, unmanaged containers and unmaintained landscapes may provide supportive environments for mosquitoes (Honório et al. 2003; Dowling et al. 2013). The influence of cryptic habitats in these areas, such as discarded tires or other artificial containers, may be difficult to quantify in GIS-based studies, as they are not detected at the resolution of most remotely sensed data available. Socioeconomic variables therefore provide a way of indirectly including the presence of these cryptic habitats in analyses.

While many examples of different types landscape-based variables being used as predictors of mosquito activity are provided above, the influence of specific variables is inconsistent and differs between studies. This is attributed to the wide variety of methods used, differences in behaviour between species and possibly populations, as well as collinearity and statistical distribution in the variables used (Sallam, Fizer, et al. 2017). Differences in key drivers of distribution across mosquito species is not overlooked in these studies, as resources allocated to control mosquito populations should focus on those most influential on pathogen transmission (or mosquito nuisance).

For example, $C x$. tarsalis are a significant vector for West Nile virus in North America (Goddard et al. 2002). Studies find different patterns of distribution between these Culex species and Ae. vexans, where the latter have been found to use understory vegetation more than any other type of habitat (Bidlingmayer 1971; Mullen 1971; Burkett-Cadena et al. 2013), and have been associated with proximity to wetlands (Moncayo et al. 2000; Trawinski and Mackay 2010; Giordano et al. 2018) and surface wetness (Shaman et al. 2002; Chuang, Hockett, et al. 2012). Both these findings are consistent with Ae. vexans as a floodwater mosquito (O’Malley 1990; Becker 2010). Conversely, Cx. tarsalis are more frequently associated with non-forested areas (Diuk-Wasser et al. 2006; Yoo et al. 2016) or agricultural and grassland regions (Nielsen et al. 2008; Chuang et al. 2011). Habitat preference in $C x$. tarsalis for these areas may reflect its adaptation to breed within polluted eutrophic waters often found in agricultural habitats (Winters et al. 2008; Eisen et al. 2010), which concentrate organic nutrients in drier conditions (Shaman et al. 2002). However, 
these findings are not homogenous across the literature, as Diuk-Wasser et al. (2006) found positive correlation between Ae. vexans and grasslands and cultivated area, and Giordano et al. (2018) found that Culex presence is also correlated with proximity to wetlands.

However, a commonality among vector distribution studies is the method used to extract variables using circular zonal areas generated around the mosquito sampling locations (Rey et al. 2006; Chuang, Hockett, et al. 2012; Burkett-Cadena et al. 2013; Bunn et al. 2019). The variation in the surroundings of different sampling locations is characterized by summary statistics, such as the percent coverage of a LULC type or the average value of a pixel-based index such as NDVI across a zonal area (Brown et al. 2008; Landau and van Leeuwen 2012).

The size of the zonal area used is at the discretion of the researchers but is generally purported to represent the flight range of the species of interests (Kolivras 2006; Deichmeister and Telang 2011). Many studies try different iterations of models with radial zones of different sizes to find the scale at which the correlation between the pertinent environmental variables and mosquito density is most influential (Diuk-Wasser et al. 2006; Chuang et al. 2011; Ferraguti et al. 2016; Lorenz et al. 2020). With certain long-flying species, such as Ae. vexans, flight ranges of several kilometres may be used as zonal area radii if there is little overlap between different zonal areas (Field et al. 2019). Otherwise, it is advisable to select a zone radius size that minimizes overlap between zonal areas, as to represent an appropriate amount of spatial heterogeneity between sampling locations (Burkett-Cadena et al. 2013). Data obtained from this method are commonly transformed to better meet expectations of normality and produce more reliable models with reduced multicollinearity in the following step (Sallam, Fizer, et al. 2017).

\section{Spatial statistics and visualization}

Several statistical tests can help users gain insight on the structure of their data prior to developing spatial models. Measures of correlation such as Spearman's rho can be used to infer relationships between the response variable and the explanatory variables (Bidlingmayer 1967; Kitron and Kazmierczak 1997), or to detect collinearity between the latter (Gleiser and Zalazar 2010; Mallya et al. 2018). Moran's I is the most common index used to test for spatial autocorrelation and is used in many mosquito distribution studies 
(Kitron et al. 1996; Ryan et al. 2004; Diuk-Wasser et al. 2006; Ferwerda and Lathrop 2009; Giordano et al. 2018). It measures the interdependence between values at different locations and produces a value between -1 and 1 . Values closer to -1 indicate that value points are significantly more dispersed than would be expected if values were distributed randomly across the landscape. When Moran's I approaches 1, it indicates the opposite (significant clustering), whereas a value near to 0 suggests that the distance between two locations has no effects on their values.

Other interpolative approaches include the use of semivariograms (Curran 1988), which are sometimes used to corroborate Moran's I, especially if kriging or interpolation is desired (Liu et al. 2013; Giordano et al. 2018). Semivariograms do not detect clusters or dispersion, but can determine the distances among values at which correlations are greatest, and can show the impact of local random effects (Ferwerda and Lathrop 2009). Cluster analysis can be achieved with statistics like the Getis-Ord *Gi (DeGroote et al. 2008; Nolan et al. 2012) and Anselin's local Moran's I (Ruiz et al. 2004; Tokarz and Novak 2018).

While these methods provide valuable information about the data's spatial structures, sophisticated spatial modeling techniques are required if forecasting or mapping is desired. Regression models are commonly used to model mosquito distribution, and linear regression models are favored by many for their ease of interpretation (Deichmeister and Telang 2011; Burkett-Cadena et al. 2013; Bunn et al. 2019). Also used are logistic models, where the response variable being modeled is expressed in discrete classes, usually binary (e.g., high/low, present/absent, etc.). As examples, these approaches include both multinomial or MaxEnt (Conley et al. 2014; Uusitalo et al. 2019) and univariate logistic regressions (Diuk-Wasser et al. 2006; Chuang, Hockett, et al. 2012). Among others, mixed models (Chuang et al. 2011), negative binomial regression (Healy et al. 2013), and decision tree models such as boosted regression trees (Conley et al. 2014) and Random Forest regressions have also been used to model species distribution based on environmental variables. As they are central to the spatial analysis discussed in Ch.4, the use of decision trees and the Random Forest machine learning algorithm will be discussed in greater detail further below. 
To determine which variables should be included in the final model, many proceed stepwise with their regressions (Landau and van Leeuwen 2012; Sallam, Michaels, et al. 2017). Some use principal components analysis (PCA) to aggregate redundant variables (Rey et al. 2006; Giordano et al. 2018), or calculate the variance inflation factor (VIF) of their model to avoid the problem of collinearity between variables (Diuk-Wasser et al. 2006; Chuang, Hockett, et al. 2012; Burkett-Cadena et al. 2013). Those proceeding stepwise must also be cognizant of the inclusion of noise variables, which may compromise the outcome of finding the "best" models (Tabachnick and Fidell 2013).

However, several of the statistical problems commonly encountered during analysis of ecological data, including those related to noise or collinearity, can be avoided with the use of decision trees, also called regression trees or classification trees. A decision tree is a supervised machine learning model that resembles a flow chart in the shape of an inverted tree, as per its namesake (Rokach and Maimon 2015). Decision trees are both descriptive and predictive, in that they can display the systematic structure of data, as well as predict the values of unsampled data (De'Ath and Fabricius 2000). At each node (branching point) in the tree, a specific predictive variable is chosen to split to the data into two groups such that the data in these groups are as homogenous as possible (Denil et al. 2014). This process is then repeated on each individual group with the remaining predictor variables until there are no more predictor variables available to split the data (De'Ath and Fabricius 2000). The first node in the tree identifies the most important predictor variable, with predictors decreasing in importance as they approach the terminal nodes or "leaves". The terminal nodes represent the predictions made by the decision tree, which can either be categorical (in classification trees) or continuous (in regression trees).

The inherent structure of decision trees safeguards against many problems often encountered in ecological data. Both numeric and categorical data can be included in the same model (Therneau and Atkinson 2013), and they are robust enough to handle missing values and non-normal distributions (De'Ath and Fabricius 2000). Due to their non-linear construction, decision trees also perform well where linear regression models fail when modelling non-linear relationships (De'Ath and Fabricius 2000). Datasets that exhibit multicollinearity, or significant correlation between predictor variables are selected against 
by the splitting procedure, as redundant variables are unable to efficiently classify the data further than covariates used at previous nodes (Therneau and Atkinson 2013). Additionally, decision trees are easily represented visually and mimic the taxonomy present in human decision-making processes, and so can be intuitively understood by others (Therneau and Atkinson 2013).

Decision trees fall short when trying to generalize to data it has not been trained with, which can result in a model that is overfit to the input data. However, a nonparametric method known as Random Forest (Breiman 2001; Cutler et al. 2007; Biau and Scornet 2016) circumvents this problem by constructing many decision trees, and taking the mean prediction of each tree. The advantages to Random Forest (RF) lie in the "bagging" of observations (named for bootstrap aggregation) used to validate each decision tree and the random selection of features from which the tree models are built.

Bagging is a procedure in which two thirds of the dataset is sampled with replacement (also known as bootstrapping) to create a training dataset for an individual decision tree. The remaining third of the data not sampled (the OOB or "out of bag" data) is then used to validate the tree model and to calculate an OOB error. This value represents the proportion of, or extent to which the predictions did not match the values in this validation data (Liaw and Wiener 2002). The differences between each OOB prediction $\left(y_{i}\right)$ and the mean of $n$ OOB predictions each $i^{\text {th }}$ observation (the OOB error) is then aggregated to calculate the mean square error (MSE) of the model predictions, as in the following equation (Liaw and Wiener 2002):

$$
M S E=n^{-1} \sum_{1}^{n}\left(y_{i}-\hat{y}_{i}^{O O B}\right)^{2}
$$

The MSE is used to gauge the Random Forest model's performance, with lower MSE indicating that on average, the trees have greater accuracy in predicting the OOB data. From here, the percent variance explained (goodness of fit) can be calculated using the total variance of the response variable $\left(\sigma^{2}\right)$ (Liaw and Wiener 2002):

$$
1-\frac{M S E}{\sigma_{y}^{2}}
$$


In addition to this bagging procedure, a subset of randomly selected predictive variables (the number set by the user) are compared to one another at each node. The variable that splits the data into the most homogeneous groups is chosen and the process repeats itself with another subset of variables at the next node. The Random Forest algorithm also estimates the importance of each individual variable in the model by computing how much the variable causes a decrease in prediction accuracy, measured as the percent increase in MSE. The number of trees permuted in the Random Forest model must be set by the user, and a greater number of trees generally results in a more stable model in terms of relative variable importance, but increases computation time (Liaw and Wiener 2002).

The robustness of RF is especially evident in circumstances where there are fewer observations than predictors (Strobl et al. 2007). It has been observed to perform well when evaluating the effects of multiple predictors operating at multiple spatial scales (Bradter et al. 2013). Nevertheless, bias can show up in model predictions if the distribution is not representative of the full range of variation in the response variable. Random Forest model predictions will tend towards the value with highest variation in the response variable Therefore it is sometimes advisable to transform the response variable to improve its distribution (De'Ath and Fabricius 2000; Ibañez-Justicia and Cianci 2015). Another caveat to its use and interpretation is that RF cannot extrapolate, it can only interpolate (Hengl et al. 2018), and so the minimum and maximum prediction values will always reflect those in the training data.

When modelling species distribution, including that of mosquitoes, RF has been shown by many to outperform traditional parametric regression methods, including competing machine learning approaches ( $\mathrm{Li}$ and Wang 2013; Kwon et al. 2015; Mi et al. 2017; Diarra et al. 2018; Mudele et al. 2019; Uusitalo et al. 2019). However, only recently has the use of RF become more common in mosquito distribution studies (Ibañez-Justicia and Cianci 2015; Kwon et al. 2015; Ferraguti et al. 2016; Ong et al. 2018; Chen et al. 2019). Once the best models are chosen, a map with predictions of the values at unsampled areas can be produced. The predicted values will represent the decisions of the majority of the trees generated by the model (Kwon et al. 2015). 
Finally, models are sometimes validated with independent data if available (DiukWasser et al. 2006; Chuang, Henebry, et al. 2012; Healy et al. 2013). While the bagging procedure inherent in RF models offer some external validation, additional validation can be achieved using "true" external data (Ibañez-Justicia and Cianci 2015). A model has good predictive ability if it is successful in modeling data from another dataset (from a different time or nearby region) with the same predictors. In mosquito distribution models, this would signify that decisions made based on the model's predictions are scientifically sound and can be generalized within reason. Additionally, models may be validated by separate analyses, where the dependent variables are measured using different representative values. If the predictions from these models show similar outcomes, this lends credence to the accuracy of these models. 


\section{CHAPTER 3 - MARK-RELEASE-RECAPTURE STUDIES}

Title: Determination of mosquito dispersal distance for evaluation of buffer zone usefulness around the city of Winnipeg

\section{0 - Abstract}

Background: Understanding the dispersal behaviour of nuisance and disease vector mosquitoes is essential to the implementation of successful mosquito abatement programs. To minimize adult dispersal, programs commonly maintain buffer zones surrounding highly populated areas, within which mosquito breeding habitats are actively managed. However, it is necessary to establish these buffer zones such that they accommodate the flight distance and behaviour of local mosquito species with respect to their environment. This study was designed to determine the average flight distances and influence of landscape features on the dispersal of mosquitoes in the Winnipeg region in central Canada, where a buffer zone is maintained by local mosquito control authorities.

Methods: Mark-release-recapture experiments (MRR) on adult mosquitoes were carried out in the summer of 2016 and 2017. Mosquitoes were marked with fluorescent dust and recaptured in New Jersey light traps for at least 11 days following their releases.

Results: Some common species in the Winnipeg region, including Aedes vexans, Culiseta inornata, and Coquillettidia perturbans were marked and recaptured in this study. Marked mosquitoes were recaptured $3 \mathrm{~km}$ or more from the release site within a few days following emergence. Female Ae. vexans are estimated to have a flight range of at least $8 \mathrm{~km}$ and possibly upwards of $15 \mathrm{~km}$. Male Ae. vexans were also observed to be capable of making flights of more than several kilometers. Female Cs. inornata movement was strongly driven by the presence of mammalian host animals. No clear influence of the effects of wind velocity or direction could be determined from the location of the recaptures, although many marked mosquitoes were recaptured in river-adjacent and forested areas indicating that landscape features with extensive vegetative cover appeared to be attractive to dispersing mosquitoes. Furthermore, these landscape features may act as corridors facilitating dispersal and routes of entry for immigrating mosquito populations. 
Conclusions: The flight ranges observed in these studies support the extent of the current mosquito control buffer zone surrounding Winnipeg. Though the object of this study was not to determine whether mosquitoes are preferentially dispersing into urban areas from outside them, the distances that marked mosquitoes travelled demonstrated their ability to fly into the city from surrounding rural municipalities. These findings also highlight the potential for vegetated areas to serve as corridors that enable long-range dispersal.

Keywords: Mark-release-recapture, mosquito, dispersal, flight distance, buffer zone, Culicidae, Aedes vexans, Culiseta inornata

\section{1 - Introduction}

Mosquitoes are pests and disease vectors of significant concern in virtually all parts of the world. Broad and pre-emptive rather than reactive mosquito control measures have been shown to reduce mosquito populations and reduce the number of local cases of mosquito-borne illness (Tomerini et al. 2011). Among mosquito control operations enacting pre-emptive measures, surveillance and treatment of larval mosquito habitat is most common. However, other measures, such as the implementation of buffer zones where active mosquito control occurs, may also be valuable in preventing adult mosquitoes from dispersing into urban areas from elsewhere. The municipal insect control branch (ICB) in Winnipeg, Manitoba allocates significant resources to mosquito control, including the surveillance and treatment of an 8 to $12 \mathrm{~km}$ buffer zone (usually $<10 \mathrm{~km}$, depending on available resources) surrounding the city. However, there is little local evidence to suggest that larviciding a buffer zone of this size effectively prevents the development of highdensity adult mosquito populations within city boundaries. Additionally, as mosquito populations are composed of multiple species, barrier zone widths should be established according to the dispersal abilities of the dominant species or those of greatest concern.

Dispersal metrics are often based on the average distances travelled by mosquitoes subject to mark-release-recapture (MRR) experiments, but can also summarize the expected maximum flight ranges for a proportion of the mosquito population (Morris et al. 1991). In a metadata analysis on the flight ranges of various mosquito species, Verdonschot and Besse-Lototskaya (2014) found that Culex tarsalis Coquillett, 1896, had a maximum average flight range of only $289 \mathrm{~m}$. They asserted that a buffer distance greater than this 
would be required to achieve $90 \%$ reduction in their breeding population. For mosquitoes that were found to have some of the greatest flight ranges, such as Aedes vexans (Meigen, 1830), they explicitly suggest that based on their average maximum flight distance of 8 $\mathrm{km}$, "a barrier zone will be useless". This assumes that the maintenance of a barrier zone that extends beyond of this distance would be impractical for most urban areas. However, the current buffer zone of 8 to $12 \mathrm{~km}$ surrounding the City of Winnipeg may be defensible in this case.

While information on flight distances is essential to consider when making decisions regarding the implementation of a buffer zone, a qualitative understanding of mosquito dispersal is also important. Current literature strongly supports an understanding of mosquito dispersal behaviour as a complex process that is broadly influenced by landscape structure, environmental conditions (e.g., temperature, humidity, wind, illumination) and species physiology (Bidlingmayer 1985; Verdonschot and BesseLototskaya 2014; Trewin et al. 2019). Areas with vegetation of sufficient height and density may act as corridors wherein mosquitoes can orient with the silhouette of continuous vegetation such as trees (Bidlingmayer and Hem 1981; Schäfer et al. 1997), especially if these are near a body of water where humidity is relatively high. Presence of standing water near these areas may signal available breeding sites, which may further affect dispersal patterns (Reiter et al. 1995).

Ideally, barriers zones should exploit these dispersal behaviours, and exhibit adverse environmental conditions, such as higher relative temperatures and lower related humidity, which strongly lower mosquito survival rates (Clements 1963; Craig et al. 1999). Open areas with little shelter from wind and heat are most likely to prevent adult females from reaching urban areas from peri-urban breeding sites. Conversely, recommendations also exist to plant "islands" of shrubs within a buffer zone to provide shelter and reduce their flight distance (Bruce-Chwat 1985; Oaks et al. 1991). It is likely that a barrier zone's width is only as effective as the secondary conditions within it that contribute to limiting mosquito dispersal.

The objective of this study was to document the dispersal of marked mosquitoes, primarily Ae. vexans, into the Winnipeg area within the province of Manitoba, Canada. We 
performed mark-release-recapture (MRR) studies to establish the effective flight ranges of many common mosquito species and to discern the influence of landscape-based variables on their dispersal. I hypothesized that adult mosquitoes would exhibit dispersal patterns driven by landscape features. I predicted that adult mosquitoes would show active (rather than wind-assisted) dispersal towards areas with higher moisture profiles, such as those with high vegetative cover, or near rivers or standing water, and that females would disperse toward areas with high densities of their preferred hosts. The findings will assist local mosquito surveillance and control efforts, and to help determine whether the current $10 \mathrm{~km}$ buffer zone around the urban perimeter of Winnipeg would be effective considering the dispersal capabilities of mosquitoes in the region.

\section{2 - Methods}

\subsection{1 - Study site}

The study site for all mark-release-recapture (MRR) experiments included the City of Winnipeg and adjacent municipalities in Manitoba, Canada. This region is approximately $110 \mathrm{~km}$ north of the Canada-United States border with North Dakota and Minnesota (Figure 1). It covers nearly $200,000 \mathrm{ha}\left(2000 \mathrm{~km}^{2}\right)$ at 230 to $240 \mathrm{~m}$ above sea level (Environment Canada 2010). The Winnipeg region occupies the northern end of the Red River valley and is drained by the La Salle, Assiniboine, and Seine rivers as its main tributaries. The Red River valley is in the Lake Manitoba plain, a transitional zone near the southern edge of the boreal plains. Prior to European settlement, it was located at the northern border of the aspen parkland and tallgrass prairie ecoregions within the prairies ecozone near the Canada-United States border (Ecological Stratification Working Group 1996; Schellenberg et al. 2016).

The prairies ecozone is the most human-altered region in Canada, with Winnipeg and the surrounding region dominated by intense mixed agricultural uses including cropland, rangeland and pasture (Ecological Stratification Working Group 1996). Forested areas north of Winnipeg mirror that of the boreal plains, and are described as "mixedwoods" with white spruce (Picea glauca, (Moench) Voss) and jack pine (Pinus banksiana, Lambert), as well as broadleaf species such as white birch (Betula papyrifera, Marshall), trembling aspen (Populus tremuloides, Michaux) and balsam poplar (Populus 


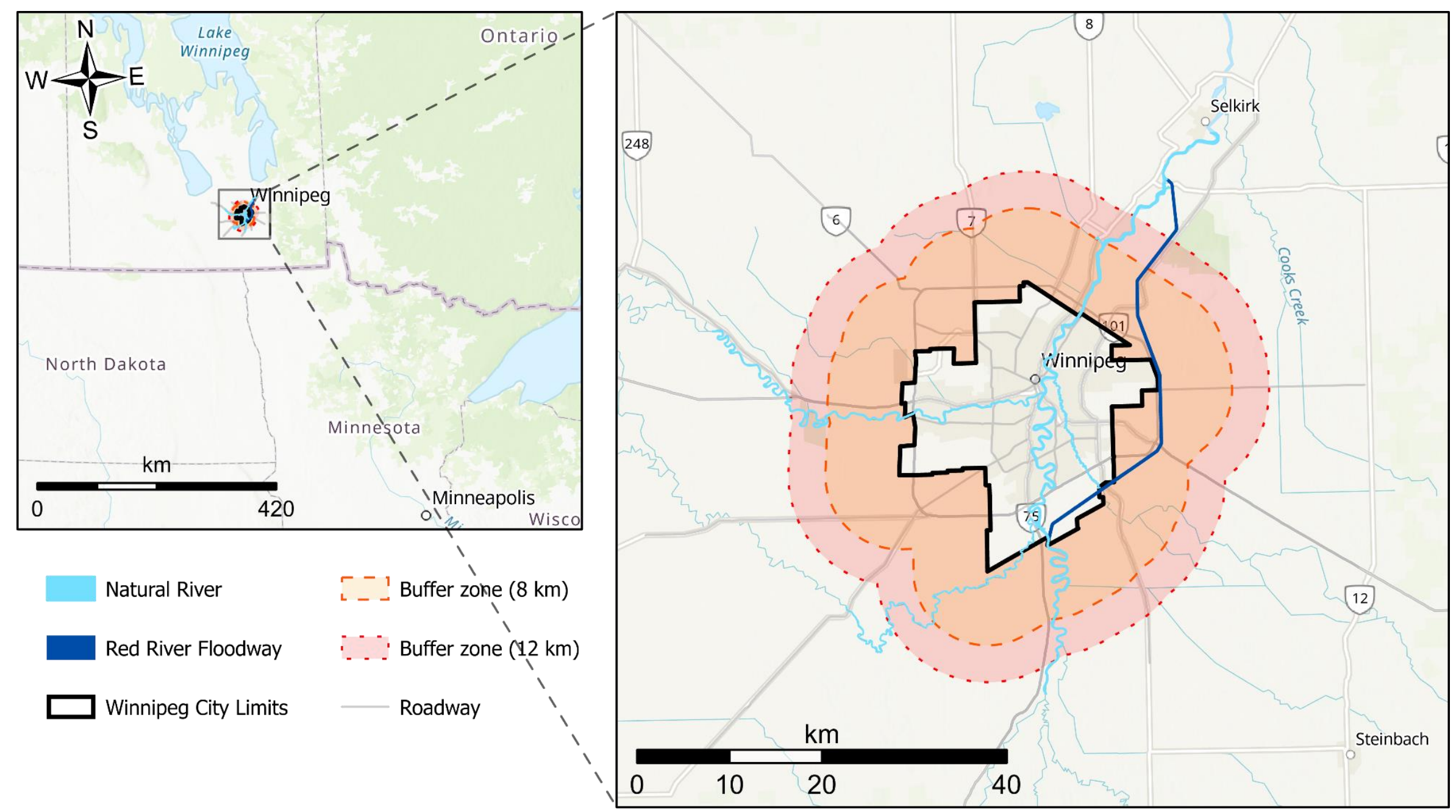

Figure 1. Region within Manitoba studied in mark-release-recapture experiments and in analysis of archival mosquito surveillance records. Mosquito control buffer zones typical extents are shown by the shaded portions. 
balsamifera, Linnaeus) (Rowe 1956; Ecological Stratification Working Group 1996). The urban forests and parklands in the area are dominated by American elm (Ulmus americana, Linnaeus, 1753), aspen (Populus L. spp.) groves, bur oak (Quercus macrocarpa, Michaux, 1801), ash (Fraxinus L. spp.), and herb and grass species common in the rest of the mixed prairie and boreal plains (Ellis 1938; Looman and Best 1987).

The region is subject to periodic flooding by run-off from higher adjacent lands and long-distance movement of water flowing north down the Red River Valley. Average annual precipitation in the Winnipeg region ranges from 450-700 $\mathrm{mm}$, with approximately two-thirds as rainfall from May through to the end of September (Ecological Stratification Working Group 1996; Environment Canada 2010). The region has a humid continental climate, with an average daily temperature between 2 and $3{ }^{\circ} \mathrm{C}$ (Ecological Stratification Working Group 1996). In January and July, the average daily temperatures are $-16.4^{\circ} \mathrm{C}$ and $19.7^{\circ} \mathrm{C}$, respectively (Environment Canada 2010).

Dark chernozemic soils are dominant in this region and are referred to as "black earth". They are weakly saline and developed on heavy lacustrine clay deposits, sometimes underlain by silt sediments (Ellis 1938). The soil's moisture-holding capacity and relatively flat topography is conducive to mechanized agricultural operations (Ecological Stratification Working Group 1996). The combination of fine soils with low permeability, heavy rainfall, and warm summer climate present optimal conditions for the establishment of mosquito populations.

\subsection{2 - Mark-release-recapture (MRR) experiments}

A series of MRR experiments were carried out during the summer of 2016 and 2017 along the southern border of the City of Winnipeg and the adjacent Rural Municipality of Ritchot. The same outdoor release site was used for both years and was located on the southern edge of the Winnipeg city limits in a City of Winnipeg tree nursery, approximately $70 \mathrm{~m}$ north of the buffer zone ( $\left.49^{\circ} 44^{\prime} 44.9^{\prime \prime} \mathrm{N}, 97^{\circ} 08^{\prime} 43.9^{\prime \prime} \mathrm{W}\right)$. Wild mosquito larvae were collected locally and reared in a series of 25 enclosed pools at this location. Rearing pools consisted of a square wooden frame $(0.86 \mathrm{~m}$ by $0.86 \mathrm{~m})$ with a maximum depth of approximately $15 \mathrm{~cm}$ (Figure 2). The frames were each lined with a polyethylene sheet and filled with non-chlorinated water to an average depth of $11 \mathrm{~cm}$ (Figure 2). Organic matter 
was added to the pools to simulate a larval habitat (Figure 3). In 2016, leaves, dry grass and other organic debris from a local riverbank park were added to the enclosures. However, due to dry weather conditions in 2017, sod was added to the enclosures instead. As an oviposition substrate, sod infusions have been shown to attract both Culex and Aedes mosquitoes (Madder et al. 1980; Lampman and Novak 1996; Jackson et al. 2005) and have previously been used in the Winnipeg area by Brust (1990) to collect Cx. tarsalis and Culex restuans Theobald, 1901 egg rafts. In both years, the sod or organic matter was completely or partially submerged in the water to varying depths. Pools were covered with a pyramidal-shaped, screened enclosure and emerging adults rested on the screen.

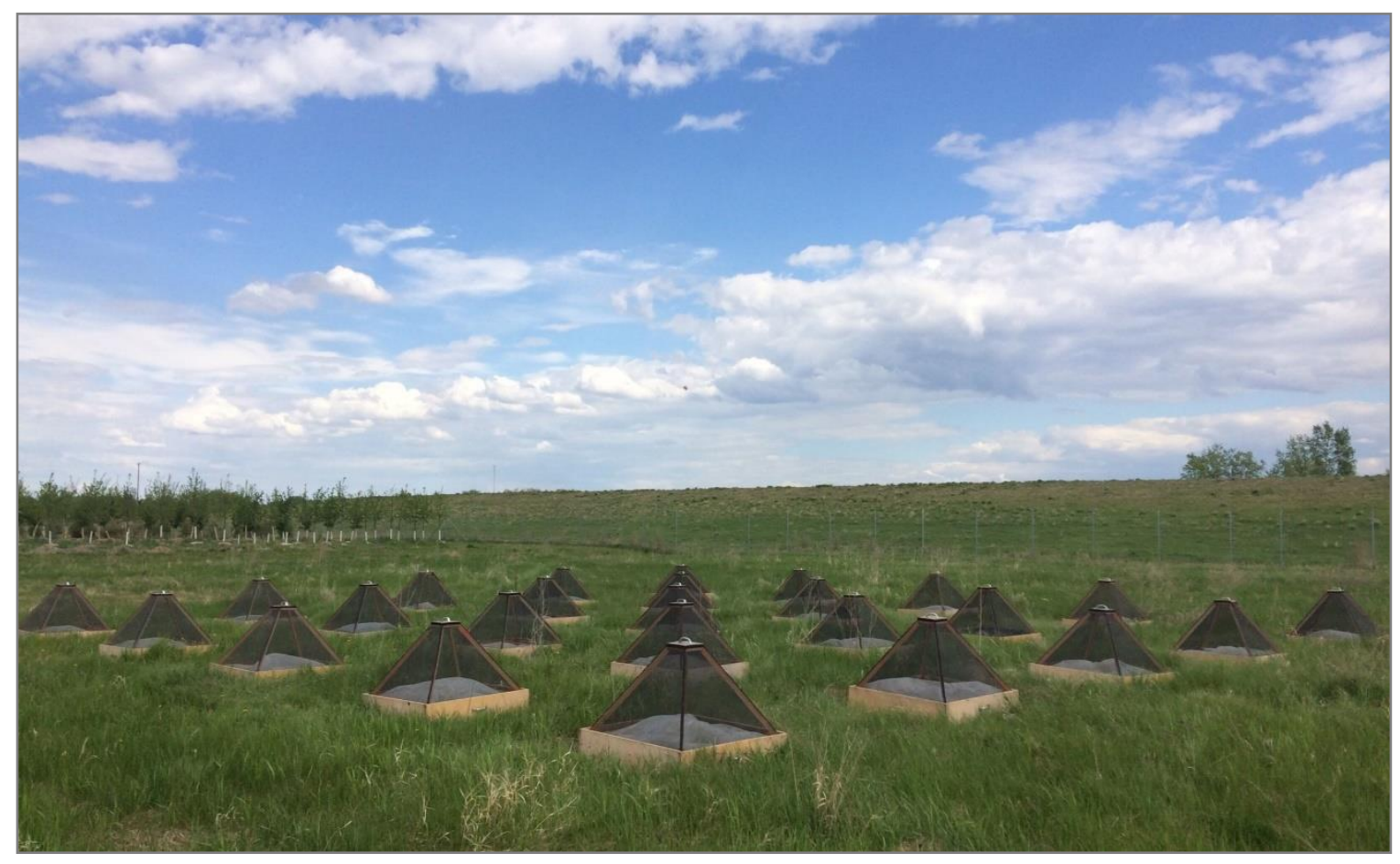

Figure 2. Empty rearing pools at the release site prior to marking (2017). Photo: M. Balcaen. 

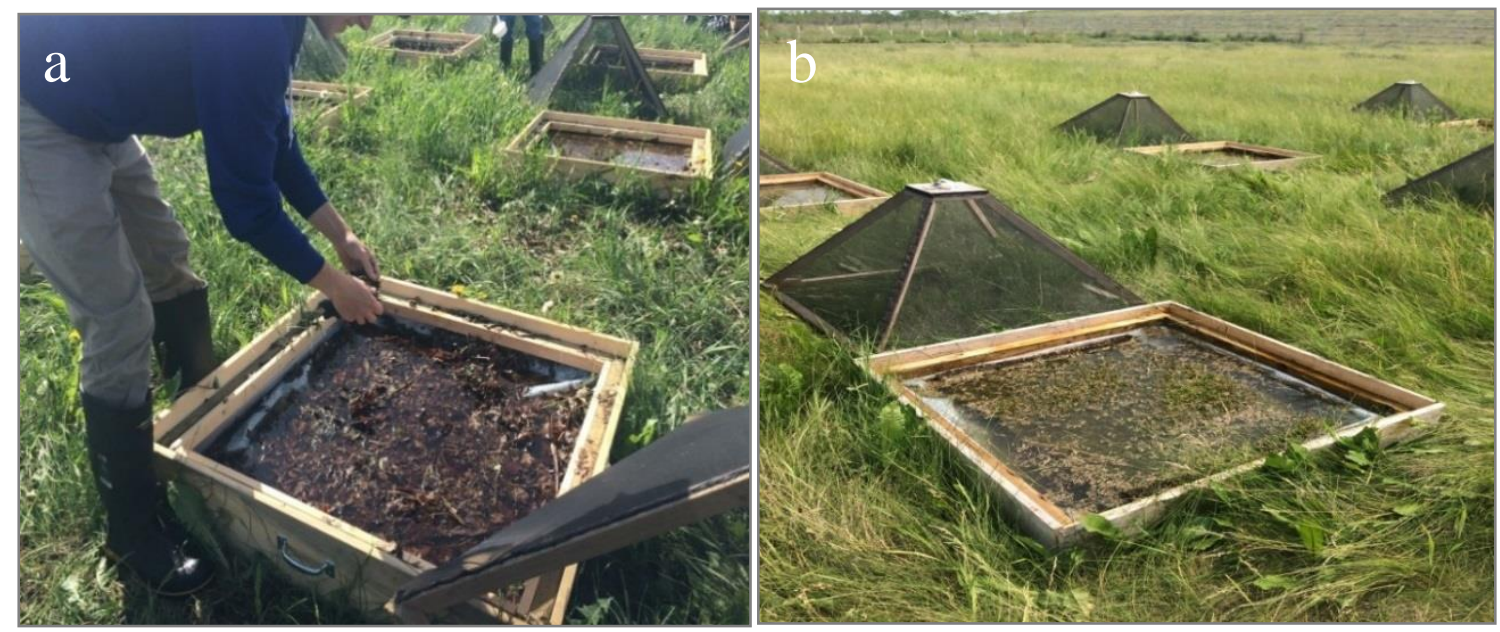

Figure 3. Water-filled rearing pools with (a) leaves and organic debris in 2016 and (b) with sod in 2017. Photo: M. Balcaen.

Once emerged, adults were trapped by sliding a polyethylene tarpaulin between the pool and the screened enclosure. The screened enclosure with the trapped adults was then moved to an area adjacent to the rearing pools and set on the ground downwind from the open pools to avoid possible contamination by the marking procedure. A second tarpaulin was placed over the side of the screened enclosure facing downwind to minimize contamination to the nearby area by marking dusts. Adults were marked through the screen with fluorescent ultraviolet (UV) pigment dust from the ECO series, which uses a formaldehyde-free resin (DayGlo Color Corp., Cleveland, $\mathrm{OH}$ ). The dust was applied with concentrated puffs from a $500-\mathrm{mL}$ polyethylene bottle fitted at the mouth with rubber tubing. Once the dust was applied, the enclosure was inverted and gently tapped to induce the adults to take flight. Mosquitoes were not blood or sugar-fed prior to their release.

Mosquitoes were recaptured in 2016 and 2017 in unbaited light traps in the region surrounding the release site. Nearly all traps were New Jersey light traps (NJLTs) (John W. Hock Company, Gainesville, FL), which are standard trap models used by most mosquito control districts in North America (Figure 4). The conical roofs of the light traps were fitted with a 25-watt light bulb that acted as an attractant. A vertical metal cylinder housed an electric fan covered by a $1 / 4$ inch mesh screen to exclude larger insects. The fan drew in mosquitoes and other smaller insects such as moths and flies that flew toward the light. A mesh funnel below the fan concentrated the insects into a collection jar with a strip of dichlorvos (formerly known as Vapona $\left.{ }^{\circledR}\right)$, an organophosphate insecticide. The traps 
ran on a general-purpose AC power supply. The traps operated continuously, except in case of occasional disruptions, such as their light bulbs burning out. During heavy rain events, samples were sometimes water-logged, rendering the mosquitoes unidentifiable, which required the samples to be discarded.

In 2017, in addition to the NJLTs, miniature battery-operated "stealth" light traps (MLTs) (John W. Hock Company, Gainesville, FL) were deployed in five trap locations where electricity was not available (Figure 5). These traps operated continuously and emitted incandescent light above a motorized fan, similar to the NJLTs. Batteries for these traps had to be exchanged at least every 2 trap nights, though battery life appeared to be inconsistent, resulting in some disruptions in collection from these traps.

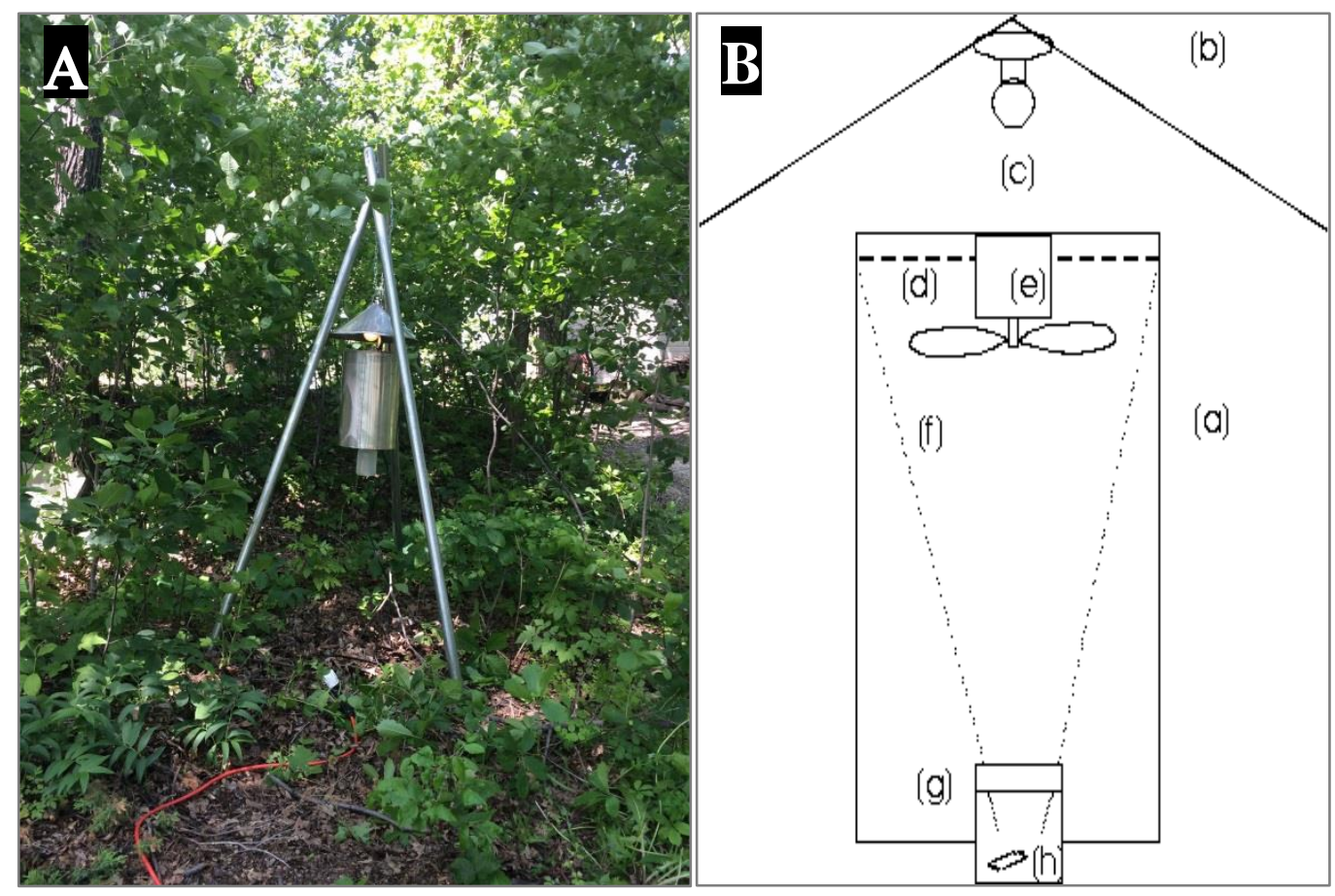

Figure 4. New Jersey light trap in the field in 4A. Trap components shown in 4B are as follows: (a) vertical metal cylinder, (b) conical roof, (c) 25-watt bulb, (d) 1/4 inch mesh screen, (e) fan, (f) mesh funnel, (g) collection jar, and (h) insecticide. Photo: M. Balcaen. Trap schematic adapted from Reinert (1989). 


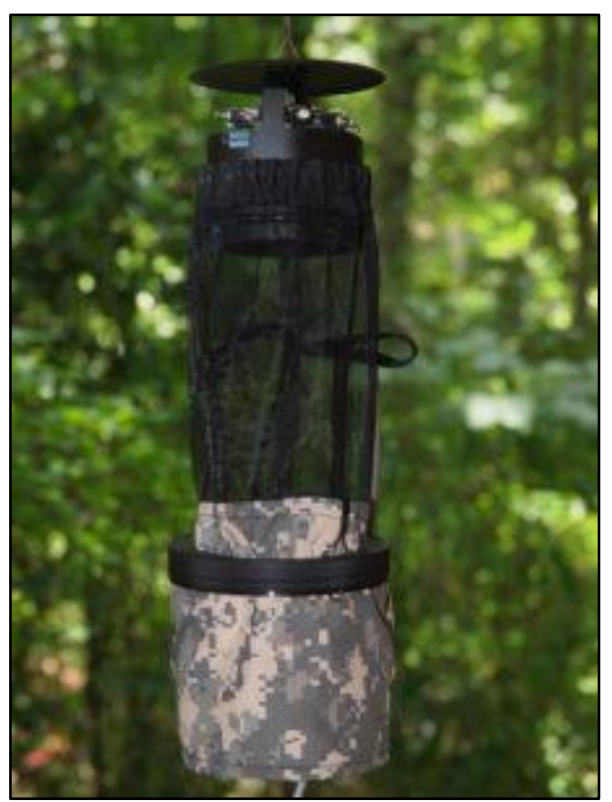

Figure 5. Miniature battery operated "stealth" light trap (MLT) hung in tree. Photo: John W. Hock Company

In both years, traps were predominantly located in grassy or tree-covered areas on residential (in private backyards) or municipal property, or in recreational areas such as public parks. See Appendix II for a full list of trap locations and their immediate surroundings. All traps from the 2017 dataset were numbered according to their distance from the release site. Traps from the 2016 dataset which remained in the same location in the 2017 dataset were given identical ID numbers. If not, they were numbered beyond the maximum number of traps present in 2017 (51).

Some traps that were deployed exclusively for the experiment were emptied each day by M. Balcaen or field assistants. These trap collections were processed by removing the mosquitoes from bycatch and recording the total number of males and females. Both marked and unmarked mosquitoes were identified to species using keys by Wood et al. (1979). All specimens were identified in 2017, but in 2016, collections were subsampled by spreading a sample across a $5 \times 5 \mathrm{~cm}$ grid and identifying all mosquitoes within a set of randomly selected of grid cells. At least $20 \%$ of each trap collection sample was identified to species using this technique, with the proportion tending toward $100 \%$ where samples contained fewer than 100 individuals. This method is considered to be accurate, and with minimal estimation error for the relative abundance of species (Jaworski et al. 2019), and 
is in concordance with the sampling approach of others (Reinert 1989). Each mosquito from all light traps in both years was visually inspected for the presence of the fluorescent marking dust using a compound microscope and external UV (black) lights in a dark room. Marked mosquitoes of both sexes were removed from collections, counted, and identified to species.

Both experiments operated concurrently with the ICB's regular mosquito surveillance period and made use of their ongoing collections from NJLTs to track mosquito dispersal. During the release periods, the frequency of collection from these traps varied according to mosquito volume. For most collection days, the numbers of female mosquitoes were recorded for only the following four species of interest: Ae. vexans, $C x$. tarsalis, Cx. restuans, Cq. perturbans (see Appendix I list of mosquito species known to be collected in Winnipeg). These collections were processed (separated and counted) by ICB staff and then inspected by M. Balcaen for fluorescent dust markings. Once per week, ICB staff identified all female mosquitoes present in one day's worth of trap collections to the species level.

\subsection{3 - 2016 MRR experiments}

In June of 2016, an MRR experiment was carried out to determine if adult mosquitoes were dispersing from the perimeter of the city toward the inner regions of Winnipeg. On May $31^{\text {st }}$ and June $1^{\text {st }}$, first and second instar mosquito larvae were collected from Dakota Park (Figure 6) in south Winnipeg in a wooded area which commonly floods in the summer ( $49^{\circ} 49^{\prime} 05.4^{\prime \prime} \mathrm{N}, 97^{\circ} 06^{\prime} 14.7^{\prime \prime} \mathrm{W}$ ). The site was selected for convenience, as ICB staff had recently reported a high volume of larvae at this site and had scheduled it for treatment with Bacillus thuringiensis israelensis (Bti) on June $2^{\text {nd }}$. Mosquitoes were collected using utility buckets and poured through a fine-meshed sieve to concentrate the larvae into a larger container. Larvae were transported to the release site and transferred into the enclosed rearing pools at the release site (Figure 6).

Decaying leaves, dried grasses, and other organic debris collected at La Barrière Park adjacent to the release site ( $49^{\circ} 43^{\prime} 12.1^{\prime \prime} \mathrm{N}, 97^{\circ} 10^{\prime} 23.1^{\prime \prime} \mathrm{W}$ ) (Figure 6) were partially submerged by non-chlorinated water in the rearing pools to simulate a natural larval habitat. Water was added as needed throughout the rearing period to compensate for 


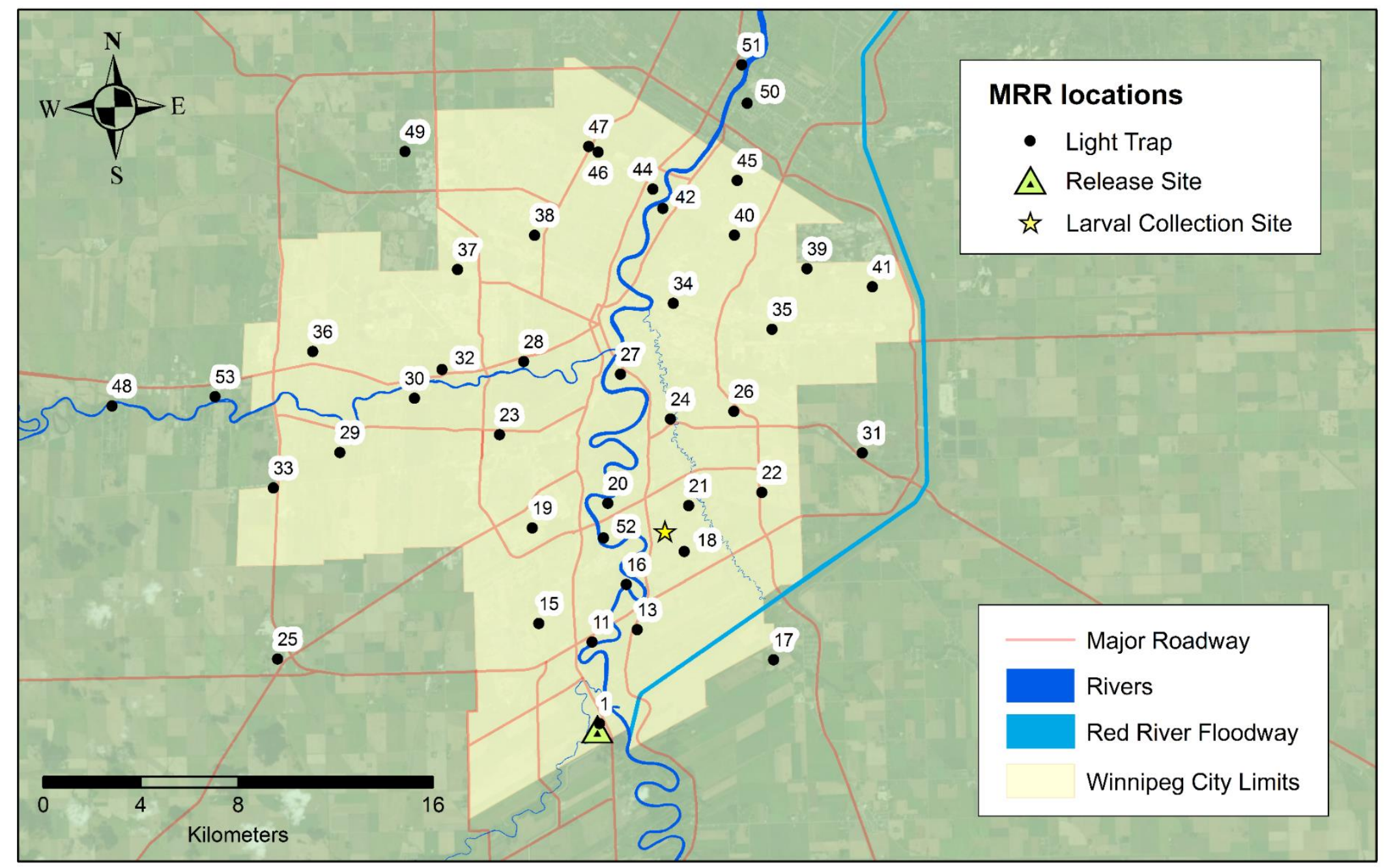

Figure 6. Location of the larval collection site, release site and numbered light trap locations for mark-release-recapture (MRR) experiments in 2016. 
evaporation. Larval numbers were estimated by taking manual counts of 3 samples from each rearing pool and extrapolating these numbers according to the water volume of each pool. Sampling from June $6^{\text {th }}$ estimated a total of 190,000 larvae. Samples from several pools were preserved for later species determination.

Prior to each marking and release occurrence, the number of adults were visually estimated in perceived units of 50 mosquitoes from each side of the screened top, and a minimum and maximum estimate was recorded (Appendix III). Adults were marked with UV fluorescent pigment dust in ECO11 Aurora Pink®. Median estimates of 19,225, $16,350,7,300$, and 7,400 adults were marked and released in the late mornings of June $11^{\text {th }}$, $13^{\text {th }}, 14^{\text {th }}$, and $17^{\text {th }}$, respectively (see Appendix III for details). A total of approximately 50,275 adult mosquitoes were marked with the pink dust. Only adults from pools with appreciable numbers of emergent adults were marked, leaving those with fewer adults to be marked later when more adults had emerged.

For a period of 44 days from June $13^{\text {th }}$ to July $25^{\text {th }}$, mosquito collections from a total of 41 NJLTs were routinely inspected (Figure 6). Marked adults were identified by microscope with the aid of two 9-watt bulb UV (black light) flashlights.

Of the 41 traps used, 37 traps were collected by the Winnipeg ICB staff as part of their standard mosquito surveillance program. These 37 traps ran continuously from May $4^{\text {th }}$ to September $28^{\text {th }}$ and were located between $5 \mathrm{~km}$ and $30 \mathrm{~km}$ from the release site (Figure 6). Trap collections and sorting were carried out daily, though no collections were made on July $1^{\text {st }}$. Prior to the release of the marked adults, I also inspected the mosquitoes collected from these traps from May $20^{\text {th }}$ to June $10^{\text {th }}$. This was to determine whether there were fluorescent pigments resembling those used for marking present in the wild mosquito population that could potentially confound the mark-release-recapture data. No fluorescent markings resembling those found on marked adult mosquitoes were observed in these inspections.

Three of the remaining four NJLTs (\#1,11, and 13) operated within $5 \mathrm{~km}$ of the release site and were collected by the author on a daily basis. Another trap (\#52) was located $8 \mathrm{~km}$ north of the release site and was collected on a similar schedule. All 4 traps were operational from June $17^{\text {th }}$ to July $7^{\text {th }}$. After July $7^{\text {th }}$, only trap \#52 remained 
operational and collection became inconsistent until the end of the collection period on July $25^{\text {th }}$ (Table 2 ). No collections were made from these 4 traps on June $28^{\text {th }}$, July $1^{\text {st }}$, and July $3^{\text {rd }}$.

Table 2. Timeline of operation for NJLTs deployed by the University of Winnipeg exclusively for MRR experiment in 2016.

\begin{tabular}{|c|c|c|}
\hline \multicolumn{2}{|c|}{ Time period } & \multirow{2}{*}{ Traps operational during this time (by trap no.) } \\
\hline Start & End & \\
\hline June 13 & June 17 & 11,13 \\
\hline June 14 & June 16 & $1,11,13$ \\
\hline June 17 & July 6 & $1,11,13,52$ \\
\hline July 7 & July 25 & 52 \\
\hline
\end{tabular}

\subsection{4 - 2017 MRR experiments}

In August of 2017, a series of MRR experiments were carried out to determine whether mosquitoes were actively dispersing towards certain dominant features on the landscape, such as water bodies and green areas. Due to record lows in mosquito numbers in 2017 (CBC News 2017 Jun 28), mosquito larvae could not be retrieved from Dakota Park or any similar location within the study area. As an alternative measure, sod was placed into the rearing pools and partially submerged in non-chlorinated water on July $20^{\text {th }}$. The pools were then left unscreened for approximately a week to attract gravid females. Additional water was added to rearing pools as necessary to compensate for evaporation or absorption by the sod. No larval samples were preserved for species determination and no estimates of larval numbers were recorded for these experiments.

Different dust colours were used in 2017 to distinguish between different release dates. Mark and release procedures were carried out on August $1^{\text {st }}, 8^{\text {th }}, 14^{\text {th }}$, and $17^{\text {th }}$. The colours used to dust mosquitoes during these release dates were UV fluorescent pigment dusts in ECO15 Blaze Orange ${ }^{\mathrm{TM}}$, ECO17 Saturn Yellow ${ }^{\circledR}$, ECO11 Aurora Pink®, and ECO19 Horizon Blue ${ }^{\mathrm{TM}}$, respectively. A total of approximately 29,000 adults were marked, with each procedure marking an average of 7,231 individuals (Table 3). Prior to marking and release on August $1^{\text {st }}$, photos were taken from each side of the screened top such that the 
number of adults could be manually counted later. This proved to be ineffective and counts for subsequent releases were done manually with clickers by two field assistants each visually inspecting two sides of the screen. Marking was carried out for all rearing pools that were observed to have resting adults (see Appendix IV for details).

For a period of 88 days from June $1^{\text {st }}$ to August $28^{\text {th }}$, I inspected all mosquitoes collected from a total of 51 light traps (Figure 7). Marked adults were identified by microscope with the aid of two 3-watt LED UV spotlight bulbs on gooseneck stands. The number and location of visible dust particles was recorded for all marked specimens.

Of the 51 traps used, 37 were NJLTs operated by the Winnipeg ICB staff as part of their standard mosquito surveillance program. These 37 traps ran continuously from May $3^{\text {rd }}$ to September $29^{\text {th }}$ and traps were located between $5 \mathrm{~km}$ and $30 \mathrm{~km}$ from the release site. Trap collections and sorting were carried out three times per week (typically Monday, Wednesday and Friday). I inspected the mosquitoes collected from these traps for 61 days

Table 3. Summary of mosquito releases in 2017.

\begin{tabular}{llc}
\hline \multicolumn{1}{c}{ Release date } & \multicolumn{1}{c}{ Dust colour used } & \# of adults marked \\
\hline August $1^{\text {st }}$ & ECO15 Blaze Orange & 1,752 \\
August $8^{\text {th }}$ & ECO17 Saturn Yellow ${ }^{\circledR}$ & 9,500 \\
August $14^{\text {th }}$ & ECO11 Aurora Pink® & 5,723 \\
August $17^{\text {th }}$ & ECO19 Horizon Blue & 11,948 \\
Total & & $\mathbf{2 8 , 9 2 3}$ \\
\hline
\end{tabular}

prior to the start of the first marking procedure (June $1^{\text {st }}-$ July $31^{\text {st }}$ ) and found no fluorescent markings resembling those used in the MRR experiments. The extended duration of this pre-release inspection period was not intentional. It was expected that inspections would only continue for a maximum of a few weeks, during which time fieldsourced larvae would be collected and reared for release, as in 2016. However, due to drought conditions, larvae could not be obtained, and alternative methods of procuring larvae were implemented in late July. 


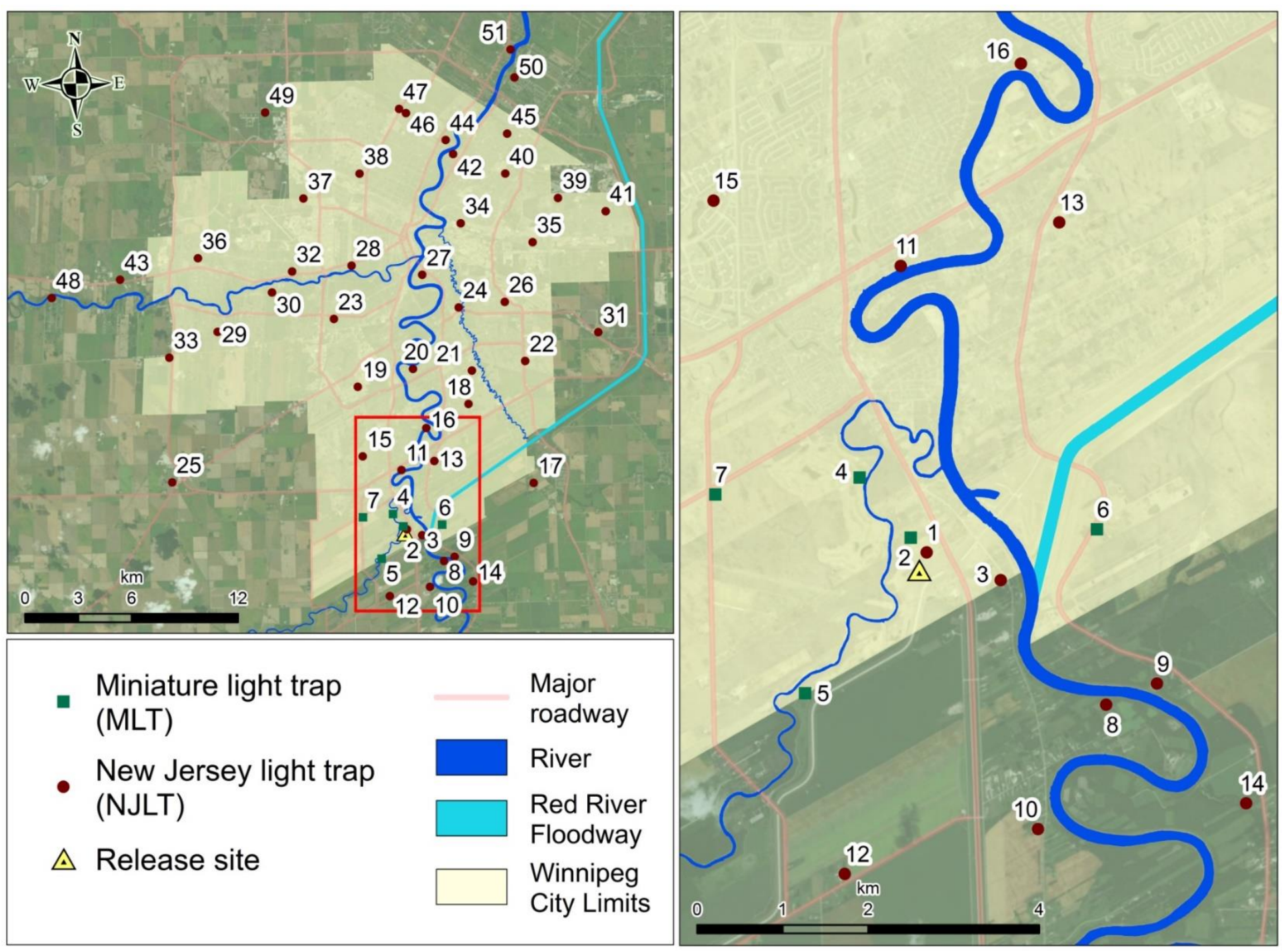

Figure 7. Location of the release site and light traps in the mark-release-recapture experiments in 2017. 
The remaining 14 traps were operated within $5 \mathrm{~km}$ of the release site and were collected by the author or by field assistants daily. A total of 9 of these traps were NJLTs, and the remaining 5 were MLTs. Individual traps were deployed throughout the period preceding the releases, and all 14 were operational by the start of the first release on August $1^{\text {st }}$ through until August $28^{\text {th }}$ (Table 4).

\subsection{5 - MRR analysis}

When calculating the average recapture rate $\left(\overline{\mathrm{x}}_{\varphi}\right)$ recaptured across all four releases in 2017, values were weighted to account for the unequal number of days for which trapping was carried out $\left(t_{r}\right)$, as well as the varying number of mosquitoes marked per release $\left(m_{r}\right)$. This was done to ensure earlier, or larger releases would not have disproportionate influence on the average capture rate. Recapture rates from individual releases were weighted according to a proportional factor $\left(\varphi_{r}\right)$. For each release, the ratio of the total trap days elapsed $\left(t_{r}\right)$ and the maximum number of trap days possible $\left(t_{\max }\right)$ was added to the proportion of mosquitoes released relative to all mosquitoes released. Proportional factors $\varphi_{r}$ were calculated using the following formula:

$$
\varphi_{r}=\frac{t_{r}}{t^{\max }}+\frac{m_{r}}{m^{\text {total }}}
$$

Similarly, when all releases were converted to a dateless time-series and consolidated, recapture results were also standardized to offset the differences due to varying numbers of marked mosquitoes across releases. For each release, the daily number of recaptured mosquitoes was divided by the relative proportion out of the total number of mosquitoes marked for a given release. The products were then normalized such that the maximum number of daily recaptures (including both sexes) for any given release was capped arbitrarily at 10 (Han et al. 2011). In other words, the highest daily recapture numbers observed for each release were standardized to 10 , while lower numbers were proportionally lower.

Data were not normally distributed, nor homoscedastic, due to a high volume of 0 s in the mosquito count data and the presence of differences in variances between marked and unmarked mosquitoes. Kendall's tau coefficient $\left(\mathrm{r}_{\tau}\right)$ was used to determine if significant 
correlations were present between numbers unmarked and marked mosquitoes in trap collections. The $r_{\tau}$ statistic is more meaningful than Spearman's rho with nonparametric data that include outliers, small sample sizes, and many tied ranks (Croux and Dehon 2010).

Table 4. Operation timeline for traps deployed exclusively by the University of Winnipeg for MRR experiment in 2017.

\begin{tabular}{|c|c|c|c|}
\hline \multicolumn{2}{|c|}{ Time period } & \multirow{2}{*}{$\begin{array}{l}\text { Operational NJLTs } \\
\text { (by trap no.) }\end{array}$} & \multirow{2}{*}{$\begin{array}{l}\text { Operational MLTs } \\
\text { (by trap no.) }\end{array}$} \\
\hline Start & End & & \\
\hline June 1 & June 6 & $1,11,13$ & 2 \\
\hline June 7 & June 8 & $1,9,10,11,13$ & 2 \\
\hline June 9 & June 10 & $1,9,10,11,12,13$ & 2 \\
\hline June 11 & June 11 & $1,3,9,10,11,12,13$ & 2 \\
\hline June 12 & June 19 & $1,3,8,9,10,11,12,13,14$ & 2 \\
\hline July 20 & July 21 & $1,3,8,9,10,11,12,13,14$ & $2,4,7$ \\
\hline July 22 & August 28 & $1,3,8,9,10,11,12,13,14$ & $2,4,5,6,7$ \\
\hline
\end{tabular}

Hourly measurements of wind velocity for the 2016 and 2017 study period were obtained from the Environment Canada Historical Data record at the Winnipeg International Airport ( $49^{\circ} 55^{\prime} 00.0^{\prime \prime} \mathrm{N}, 97^{\circ} 14^{\prime} 58.0^{\prime \prime} \mathrm{W}$ ), $20 \mathrm{~km}$ north of the release site. While temperature, precipitation and other meteorological variables may influence the volume of mosquitoes that disperse and are observed in trap collections, it was the role of physical landscape features in mosquito dispersal that was of interest in these experiments. As such, no other meteorological variables were included in the analysis. Information about daily and nightly temperature, relative humidity wind velocity, and total daily precipitation for both study years can be found in Appendices V and VI.

To compare wind speeds between periods throughout the MRR experiments, wind data were log-transformed to fit the assumption of homogeneity of variances across periods (determined by Levene's test). As the data could not be transformed to fit a normal distribution, a Kruskal-Wallis $\mathrm{H}$ test, followed by Dunn's test (with Bonferroni adjustment), was used to identify specific differences between wind speeds across periods. 
Net dispersal distance (straight-line distance between the release site and trap location for each recaptured mosquito) and bearing was measured using Google Earth Pro. Recapture data were transformed to compensate for unequal trap densities at increasing distance intervals according to the following procedure (Lillie et al. 1981; Brenner et al. 1984; Lillie et al. 1985; Morris et al. 1991).

Each trap was assigned to a circular area or "annulus" $\left(\mathrm{A}_{\mathrm{i}}\right)$ at distance $D_{i}$ from the release site. Concentric circles of annuli around the release site were each separated by a fixed distance. Each annulus had an inner radius $(a)$ and an outer radius $(b)$, with the median distance between them used as the dispersal distance $\left(D_{i}\right)$ of the mosquitoes recaptured in the traps within a given annulus (Figure 8).

$$
D_{i}=\frac{a+b}{2}
$$

This correction procedure used an annular spacing of $1.8 \mathrm{~km}$ in 2016, as it was the smallest interval in which each annulus would host at least one trap. In 2017, intervals of $1.8 \mathrm{~km}$ were also used, but an alternate analysis using $0.5 \mathrm{~km}$ annuli was also carried out to investigate whether similar results would be obtained. This second analysis excluded all recaptures beyond a $5 \mathrm{~km}$ radius, as not all $0.5 \mathrm{~km}$ annuli contained one or more traps beyond this distance.

To calculate a correction factor $\left(C F_{i}\right)$ for the transformation, the area within each annulus $\left(A_{i}\right)$ was divided by the total area $\left(A_{T}\right)$ covered by all annuli and multiplied by the total number of traps used within the experiment $\left(N_{T}\right)$.

$$
C F_{i}=\frac{A_{i}}{A_{T}} \times N_{T}
$$

This correction factor was subsequently used to calculate the expected number of recaptures $\left(E R_{i}\right)$ which would be expected for each annulus if recapture effort had been the same in each annulus. If a trap site fell on a line between dividing adjacent annuli, $\mathrm{a}^{1} / 2$ trap was reported among the total traps in each annulus $\left(N_{i}\right)$ and its observed recaptures $\left(O R_{i}\right)$ were split equally between them. 


$$
E R_{i}=\frac{O R_{i}}{N_{i}} \times C F_{i}
$$

To determine the mean distance traveled (MDT) by recaptured mosquitoes, the expected number of recaptures from each annulus was multiplied by the annulus distance $\left(D_{i}\right)$ and their products were summed for all annuli and divided by the total number of estimated recaptures $\left(E R_{T}\right)$.

$$
M D T=\frac{\sum_{k=i}^{n} E R_{i} \times D_{i}}{E R_{T}}
$$

The cumulative numbers of transformed or "corrected" ERs with increasing distance from the release site were regressed against the log-transformed distance. The regression line obtained allowed for the determination of flight range $(F R)$ for a proportion of the recaptured populations. For example, $F R_{90}$ describes the flight range for $90 \%$ of mosquitoes released. Mathematically, $F R_{50}$ is the median distance travelled and can be compared to the MDT (Morris et al. 1991). Note that unless specified as an MDT value, other distance values reported in the results are calculated without any correction for trap density.

If we assume that dispersal activity is greatest when mosquitoes are nectar- or hostseeking, as they would be upon release, MDT and $F R$ values become less meaningful as mosquitoes age. As such, these values were calculated using the corrected $E R$ values obtained after both 3 days or 7 days following the final release (or final release date for 2016) and those obtained over the complete duration of the studies. The 3-day and 7-day values describe "initial" flight ranges and mean dispersal distances (MDTs) that reflect the period of high dispersal activity following release. Those calculated using all recaptures for the study periods describe "potential" lifetime flight capabilities. Values calculated using recaptures over longer periods of time have larger sample sizes and power but may misrepresent flight capabilities as the effects of other factors such as weather and age accumulate over time. Additionally, "potential" lifetime flight capabilities may underestimate the full extent of a mosquito's dispersal over their lifetime, as mosquitoes are removed from the population before their natural death. 


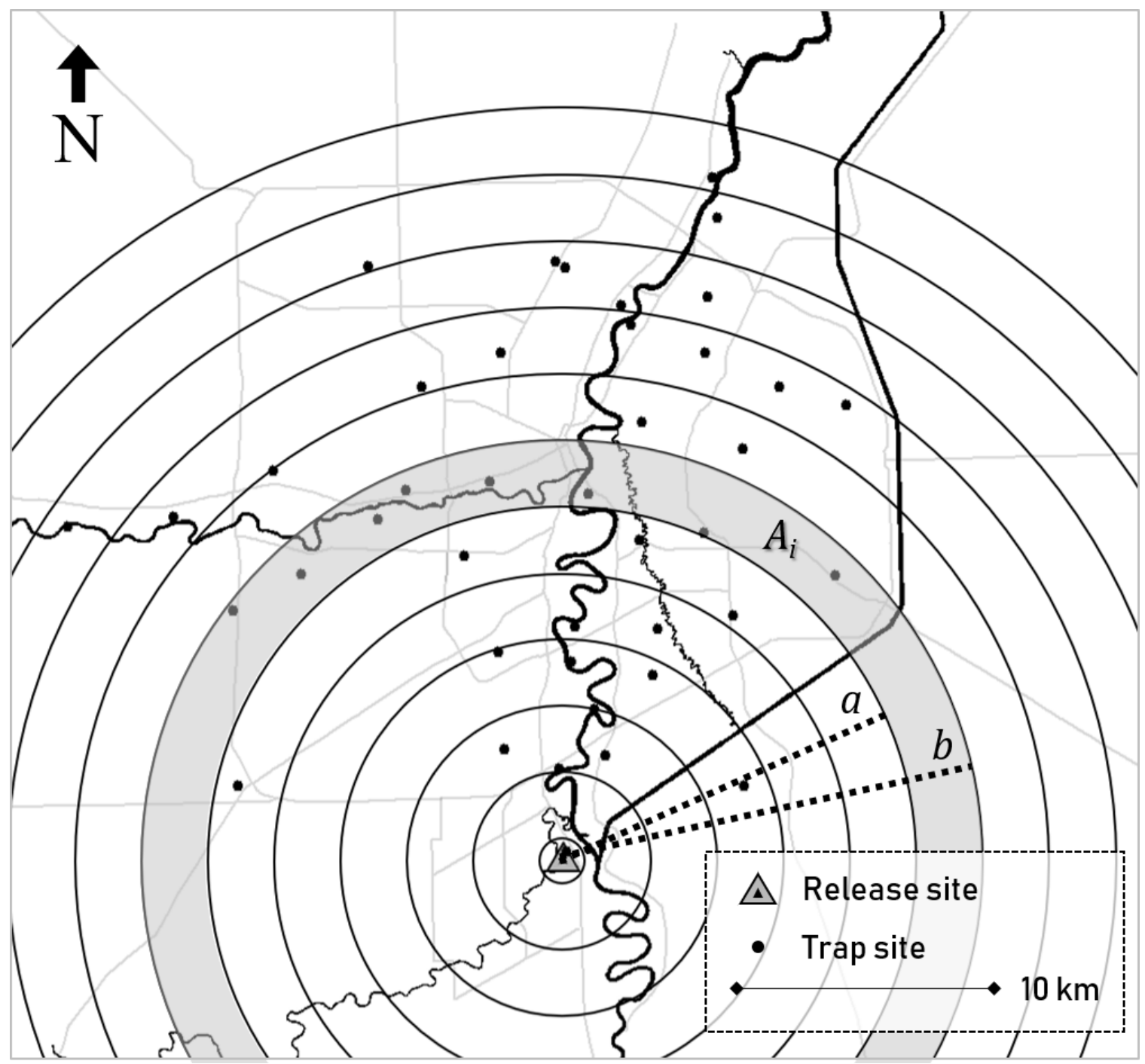

Figure 8. An example schematic of trapping annuli within the study area. $A_{i}$ refers to the area of the annulus, while $a$ and $b$ refer to the radius of the inner and outer of the annulus, respectively.

Since all traps in 2016 were within a $146^{\circ}$ angle from the release site (located between a bearing of $284^{\circ}$ and $\left.70^{\circ}\right)$, the total area of the trapping area $\left(A_{T}\right)$ and of each annulus $\left(A_{i}\right)$ was multiplied by a factor of 0.406 (146/360). These values were used to calculate the appropriate correction factors given the extent of the trap coverage. A similar correction was made in 2017 to reflect the largest "blind spots" observed in the extent of the trap coverage. The immediate trap coverage surrounding the release site was much greater in 2017; therefore, a coverage correction factor of 0.728 was used to reflect a combined $262^{\circ}$ of trap coverage. This correction excluded the combined area between the 
bearings of $284^{\circ}$ and $222^{\circ}$, and $159^{\circ}$ and $195^{\circ}$ with no trap coverage. Corrections affected the number of expected recaptures calculated at different distances but did not affect the MDT or $F R$ values calculated. Version 3.6.1 of R (R Core Team, Vienna, Austria) was used for these analyses.

\section{3 - Results}

\subsection{0 - Summary of mosquito collections during MRR experiments}

The number of mosquitoes which could be marked and recaptured was limited by precipitation, which greatly affected mosquito population numbers during the experimental flight periods in 2016 (June $11^{\text {th }}$ to July $25^{\text {th }}$ ) and 2017 (August $1^{\text {st }}$ to August $28^{\text {th }}$ ). In 2016, the temperature from early May to late September averaged $17.1^{\circ} \mathrm{C}$, and a total of $342 \mathrm{~mm}$ of precipitation was recorded. In comparison, in 2017 the average temperature was $16.3^{\circ} \mathrm{C}$, and a total of $222 \mathrm{~mm}$ of precipitation was recorded for this period (Environment Canada 2019).

During the 44-day experimental study period in 2016, $165.1 \mathrm{~mm}$ of rain fell. This was nearly 22 times more than the $7.6 \mathrm{~mm}$ of rainfall recorded during the 27-day study period in 2017. Subsequently, the average number of mosquitoes collected per trap night in $2016(\mathrm{M}=32, \mathrm{SD}=72)$ was over five times larger in comparison to those in $2017(\mathrm{M}=$ $6, \mathrm{SD}=18$ ) (Table 5). Female mosquitoes also made up a greater proportion of total trap collections in $2016(80.9 \%)$ than in 2017 (72.1\%) (Table 5).

The average daily and nightly temperatures during 2016's study period was $20.8^{\circ} \mathrm{C}$ $(\mathrm{SD}=3.7)$ and $16.2^{\circ} \mathrm{C}(\mathrm{SD}=3.5)$. Similarly, the study period of 2017 had a daily average of $18.7^{\circ} \mathrm{C}(\mathrm{SD}=6.1)$ and a nightly average $13.0^{\circ} \mathrm{C}(\mathrm{SD}=4.2)$. See appendices $\mathrm{V}$ and VI for complete climate information for both study periods.

\subsection{1 - Mark-release-recapture experiments of 2016}

Of an estimated 50,275 mosquitoes marked in 2016, 79 individuals were recaptured, resulting in a recapture rate of $0.16 \%$. Since releases were not distinguished by date, the length of the recapture period was between 39 to 44 days (median 42 days). Marked recaptures consisted of 65 females (82.3\%) and 14 males (17.7\%). Five different 
Table 5. Summary of trap collections in 2016 and 2017. Climate figures shown are calculated from the start of May to the end of September. Most traps ran continuously for 5 months from May $4^{\text {th }}$ to September $28^{\text {th }}$ (148 days) in 2016, and May $3^{\text {rd }}$ to September $29^{\text {th }}$ (150 days) in 2017. Numbers include recaptured individuals. Standard deviations are shown following means. See appendices V and VI for complete climate information for both study periods.

\section{Total number of traps}

\section{Females}

Total collected

142,423

$26 \pm 58$

Mean collected per trap/night

Males

Total collected

Mean collected per trap/night

\section{Total}

Total mosquitoes collected

176,117

Mean mosquitoes collected per trap/night

\section{Climate}

Mean temperature $\left({ }^{\circ} \mathrm{C}\right)$

Mean relative humidity (\%)

Mean daily precipitation (mm)

Total precipitation $(\mathrm{mm})$

$32 \pm 72$
$17.1 \pm 5.7$

$70 \pm 20$

$2.4 \pm 5.2$

342
41

28,189

$4 \pm 15$
10,926

$6 \pm 21$ $2 \pm 6$

$6 \pm 18$

$16.3 \pm 6.1$

$66 \pm 21$

$1.6 \pm 4.3$

222 
species were identified among the recaptures (Table 6), with Ae. vexans being the most abundant. These included among them 55 females (81\% of all Ae. vexans recaptured) and 13 males (19\%). The five species were observed in similar proportions within the wild population $(n=24,343)$ collected during the same period. Across daily trap collections, the number of wild mosquitoes captured was significantly correlated with the number of marked mosquitoes recaptured $\left(r_{\tau}=3.8, p<0.001\right)$. This trend was consistent for both female $\left(r_{\tau}=3.8, p<0.001\right)$ and male mosquitoes $\left(r_{\tau}=3.2, p<0.001\right)$.

Larval samples ( $\mathrm{n}=188$ individuals total) obtained from 14 rearing pools were all identified as Ae. vexans larvae despite nearly $14 \%$ of recaptured mosquitoes being identified as other species (Table 6). Other species were not captured in numbers significant enough to draw conclusions about their dispersal behaviours.

Table 6. Species and sex breakdown of recaptured mosquitoes in 2016. Includes composition of wild (unmarked) mosquito collection $(n=24,343)$ for collections identified to species in full for the MRR period (June $13^{\text {th }}$ to July $25^{\text {th }}$ ) for comparison. Percentages are rounded and may not add up to 100 .

\begin{tabular}{|c|c|c|c|c|c|c|c|}
\hline & 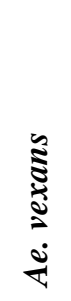 & 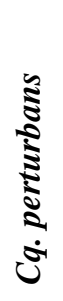 & 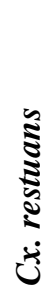 & 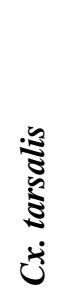 & 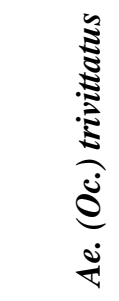 & 㿣 & $\stackrel{\pi}{\theta}$ \\
\hline Total recaptured & 68 & 7 & 1 & 1 & 1 & 1 & 79 \\
\hline no. females & 55 & 7 & 1 & 1 & 1 & 0 & 65 \\
\hline no. males & 13 & 0 & 0 & 0 & 0 & 1 & 14 \\
\hline$\%$ of recaptures & 86 & 9 & 1 & 1 & 1 & 1 & \\
\hline$\%$ of wild captures & 91 & 4 & 2 & 1 & $<0.1$ & $<1$ & \\
\hline
\end{tabular}

Over $50 \%$ of mosquitoes recaptured were collected by June $26^{\text {th }}$ (15 to 20 days following release) (Figure 9). The remaining recaptures were collected in the 29 days that followed until trapping was concluded on July $25^{\text {th }}$ (39 to 44 days following the releases). Recaptures came in two "waves": the first wave was between June $14^{\text {th }}$ and $21^{\text {st }}$ (5 to 10 


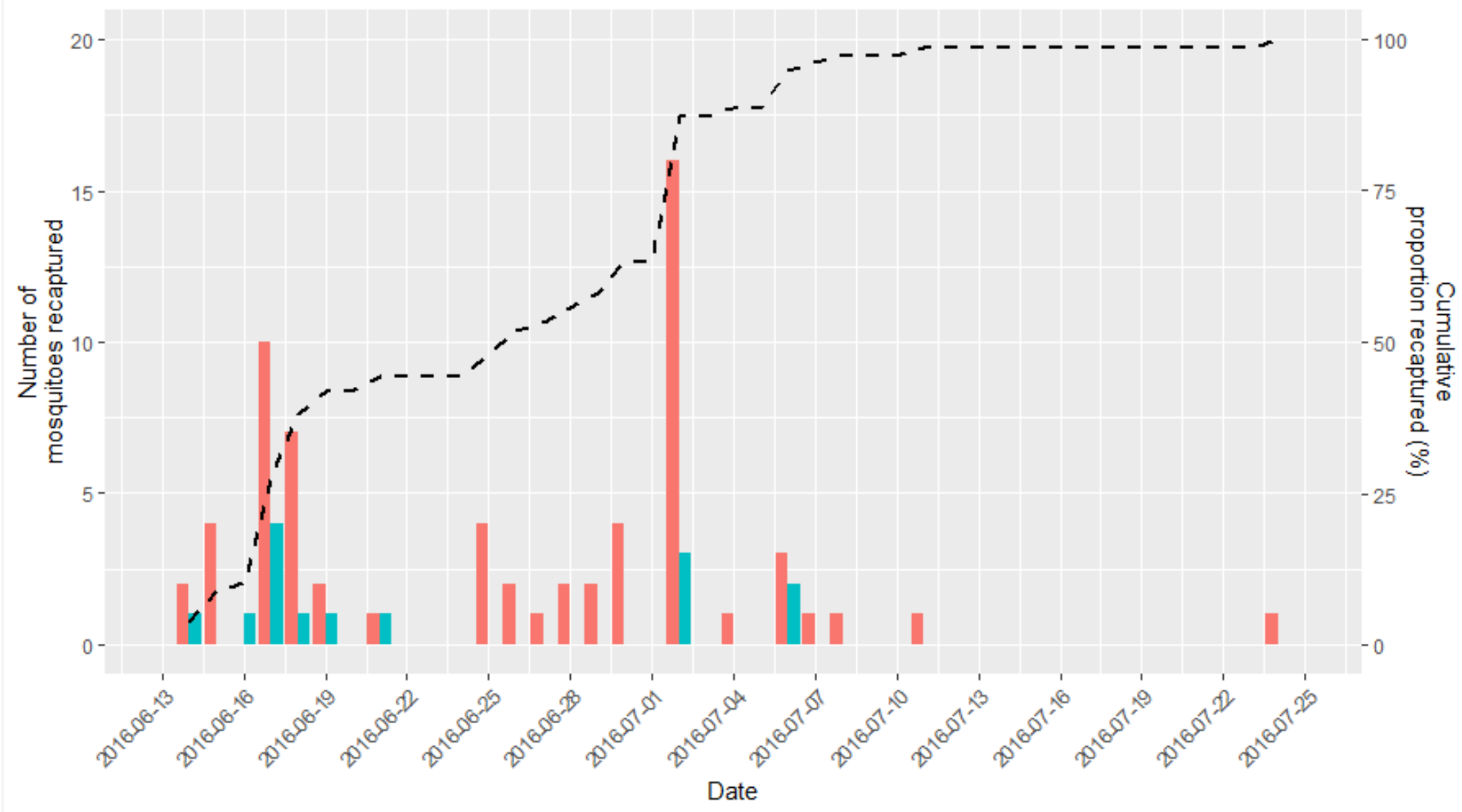

Figure 9. Daily breakdown of recaptured mosquitoes by day for 2016. Female specimens are indicated by red bars, male specimens are indicated by blue bars. The dashed line indicates the cumulative proportion of mosquitoes recaptured throughout the study period. Releases were on June $11^{\text {th }}, 13^{\text {th }}, 14^{\text {th }}$, and $16^{\text {th }}$. See appendix VII for raw data. 
days following release); the second wave between June $25^{\text {th }}$ and July $7^{\text {th }}$ (21 to 26 days following release). Only one mosquito was recaptured after July $11^{\text {th }}-$ it was recaptured on July $24^{\text {th }}, 38$ to 42 days following release (Figure 9). Daily recapture numbers of more than five mosquitoes only occurred on June $17^{\text {th }}, 18^{\text {th }}$, and July $2^{\text {nd }}$, after a median of 4-, 5- and 19-days following release, respectively. The number of males and females recaptured per day were significantly correlated $\left(r_{\tau}=\right.$ $3.4, p<0.001)$. See Appendix VII for a daily breakdown of recaptured marked mosquitoes.

With approximately one marked mosquito for every 1400 mosquitoes trapped, there were similar ratios for males and females. Trap site 1 (which was nearest to the release site) had the highest ratio of marked mosquitoes to unmarked mosquitoes, at 1 to 50 . This ratio was consistent between the sexes.

The maximum distance at recapture (dispersal distance) observed was 26.4 $\mathrm{km}$ for one female $C q$. perturbans individual at trap \#50, which was the second furthest trap north of the release site. The maximum dispersal observed for males was by an Aedes vexans individual at $7.8 \mathrm{~km}$. The furthest voyage made by an Ae. vexans was made by a female that was recaptured at $17.7 \mathrm{~km}$ from the release site. See Appendix VIII for a breakdown of recaptured marked mosquitoes in 2016 by distance from release site.

Over $95 \%$ of all recaptured mosquitoes were trapped within $8 \mathrm{~km}$ of the release site, with over 70\% recaptured in the two traps (\#1 and 11) located within $4 \mathrm{~km}$ of the release site (Figure 10). Three outliers were trapped 10, 18 and $26 \mathrm{~km}$ from the release site in traps 21,35 and 51 , after a median of 4,12 , and 15 days following release, respectively.

A total of seven traps recaptured marked mosquitoes in 2016. Only two of these traps (\#11 and \#52) were in areas shaded by trees, whereas the five remaining traps (\#1, \#13,\#21,\#35, and \#50) were all in more open areas above grassy or herbaceous ground cover. The two shaded traps (\#11 and \#52) collected $46 \%$ of all recaptured mosquitoes (37 individuals) and exactly half of all Ae. vexans. Both traps were the only ones directly adjacent $(<100 \mathrm{~m})$ to the Red River as well. Their 

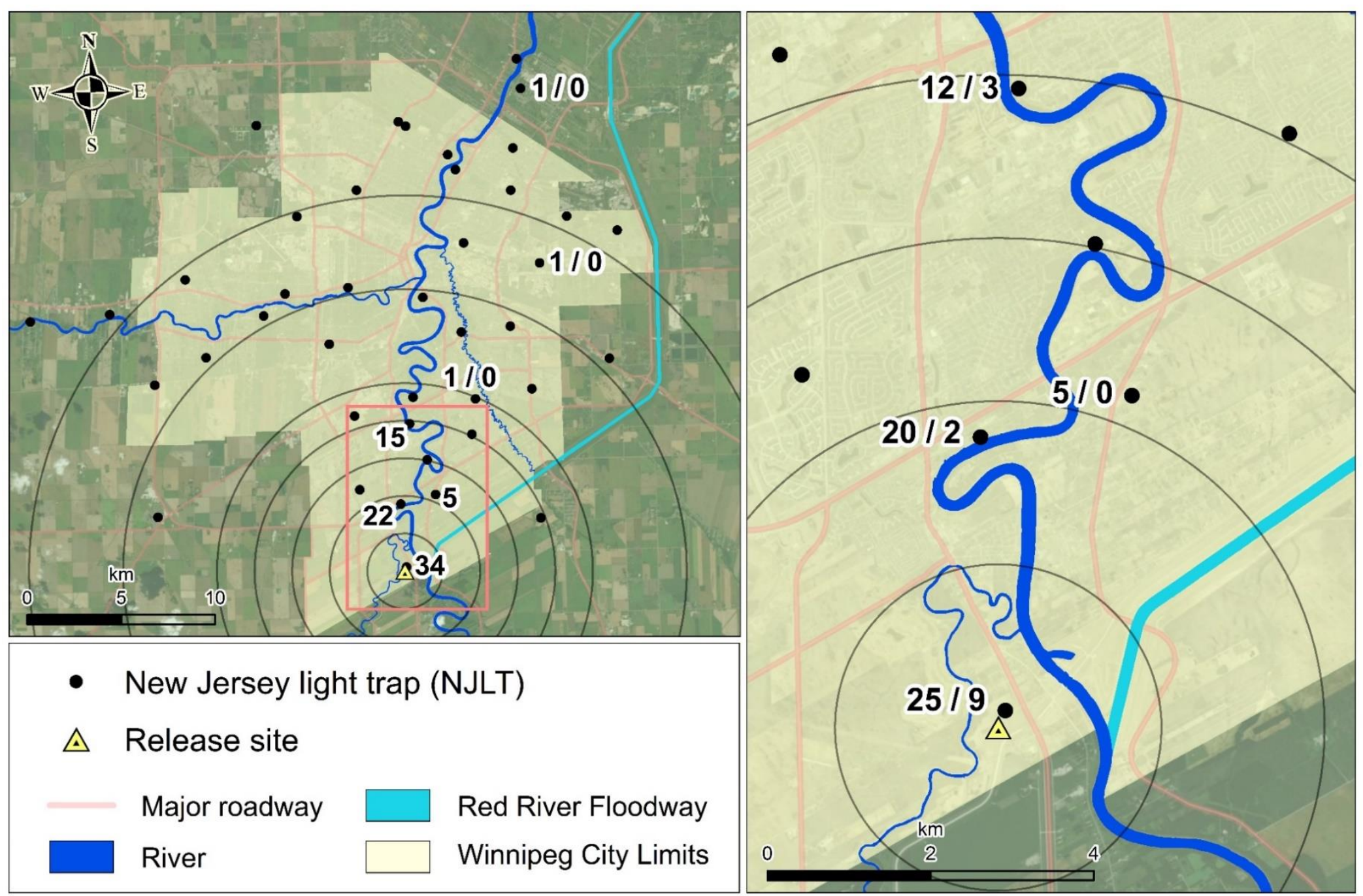

Figure 10. Recovery pattern of recaptured mosquitoes in 2016. Numbers near each trap marker denote the number of markedrecaptured females and males, respectively. Annuli are separated by $2 \mathrm{~km}$ for the first $10 \mathrm{~km}$, then by $5 \mathrm{~km}$. 
combined recapture numbers were only exceeded by trap \#1, which was closest to the release site. This trap recaptured $43 \%$ of all recaptured mosquitoes (34 individuals), and $41 \%$ of all Ae. vexans.

All three of the traps that recaptured mosquitoes more than $8 \mathrm{~km}$ north of the release site (traps \#21, \#35 and \#50, 1 individual each) were located on the east side of the Red River in grassy or herbaceous environments in residential properties. Two of these traps were relatively close to a waterway: trap \#21 was $450 \mathrm{~m}$ from the Seine River and trap \#50 was $670 \mathrm{~m}$ from the Red River. Traps \#35 was nearly $5 \mathrm{~km}$ from any waterway. Both males and females were observed dispersing at least $6 \mathrm{~km}$ from the release site within the first three days (Figure 11 and 12). For females, this trend persisted throughout the remainder of the first week.

By the end of the first two-week period following release, a female Cq. perturbans individual had reached a trap (\#50) near the edge of the trapping extent in the north. In the third week, nearly as many females were recaptured in traps up to $8 \mathrm{~km}$ from the release site in comparison to the first week, in what is referred to here as the "second wave" (Figure 11). From the start of the fourth week to the end of the study period, recapture numbers were much lower but still observed in traps up to $8 \mathrm{~km}$ of the release site. The only mosquito recaptured beyond the first four weeks was one female Ae. vexans individual, observed in trap \#52, nearly $8 \mathrm{~km}$ from the release site. Male recaptures were consistently lower regardless of time interval and were not observed beyond the 6 to $8 \mathrm{~km}$ annulus (Figure 12). See Appendix IX for a complete list of all recaptured mosquitoes, their recapture dates, and trap locations.

The prevailing wind direction and speed during the study period in 2016 was variable (Figure 13). The week following the first release (June 11 to 17) saw strong easterly winds, with predominant winds originating from the north and southeast. Westerly winds prevailed during the second week (June 18 to 24), which gave way to predominantly west and southwesterly winds accompanied by strong northwesterly winds during the third week (June 25 to July 1). Southerly winds prevailed for the remainder of the study period. The average wind speed throughout the study period was $4.1 \mathrm{~m} / \mathrm{s}(\mathrm{SD}=2.0)$. There were significant differences in wind speed between some of the four periods shown (Figure 14). 


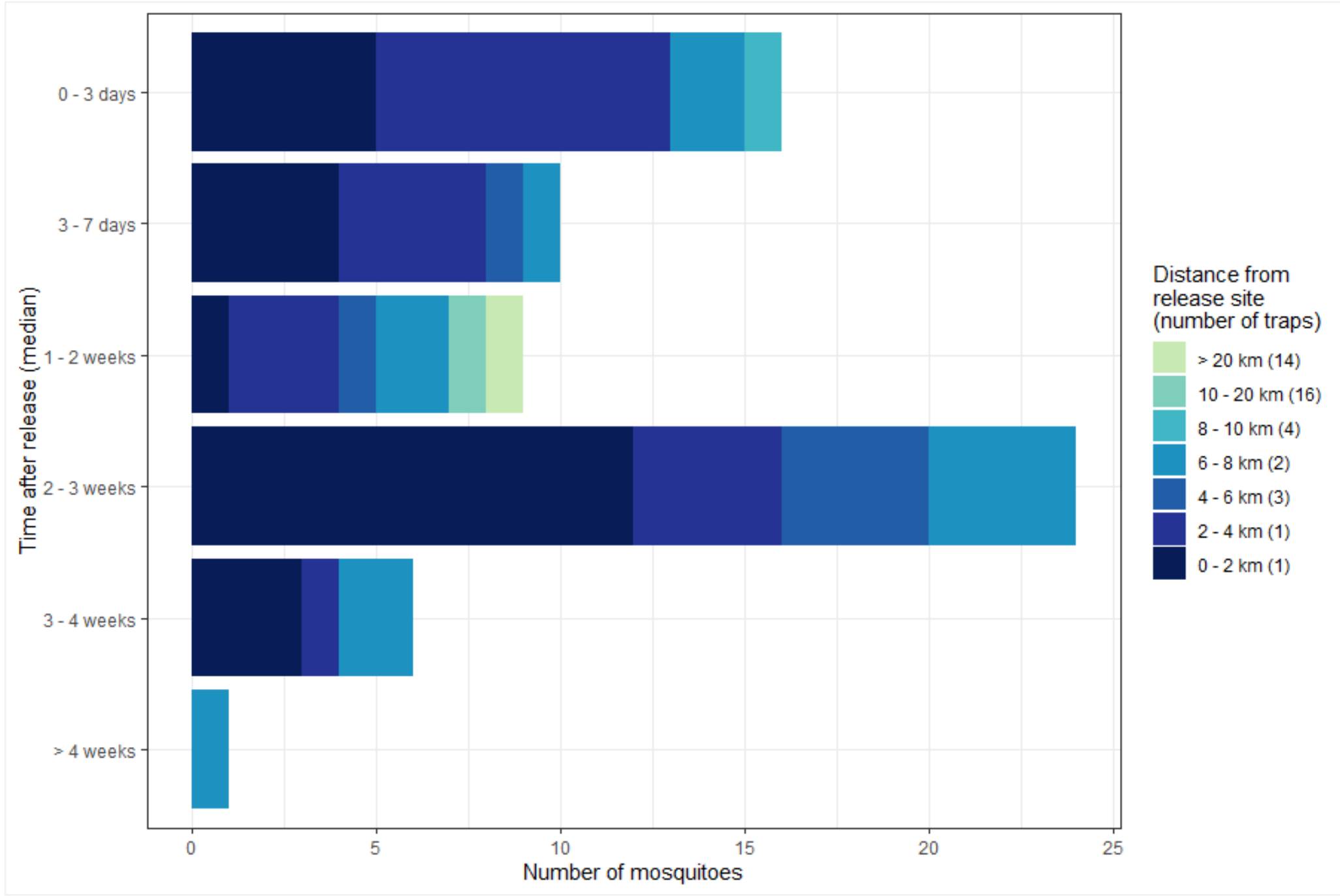

Figure 11. Number of recaptured female mosquitoes blocked by dispersal distance and time interval following release (median days used) in 2016. Number of traps in each distance block is shown in legend. 


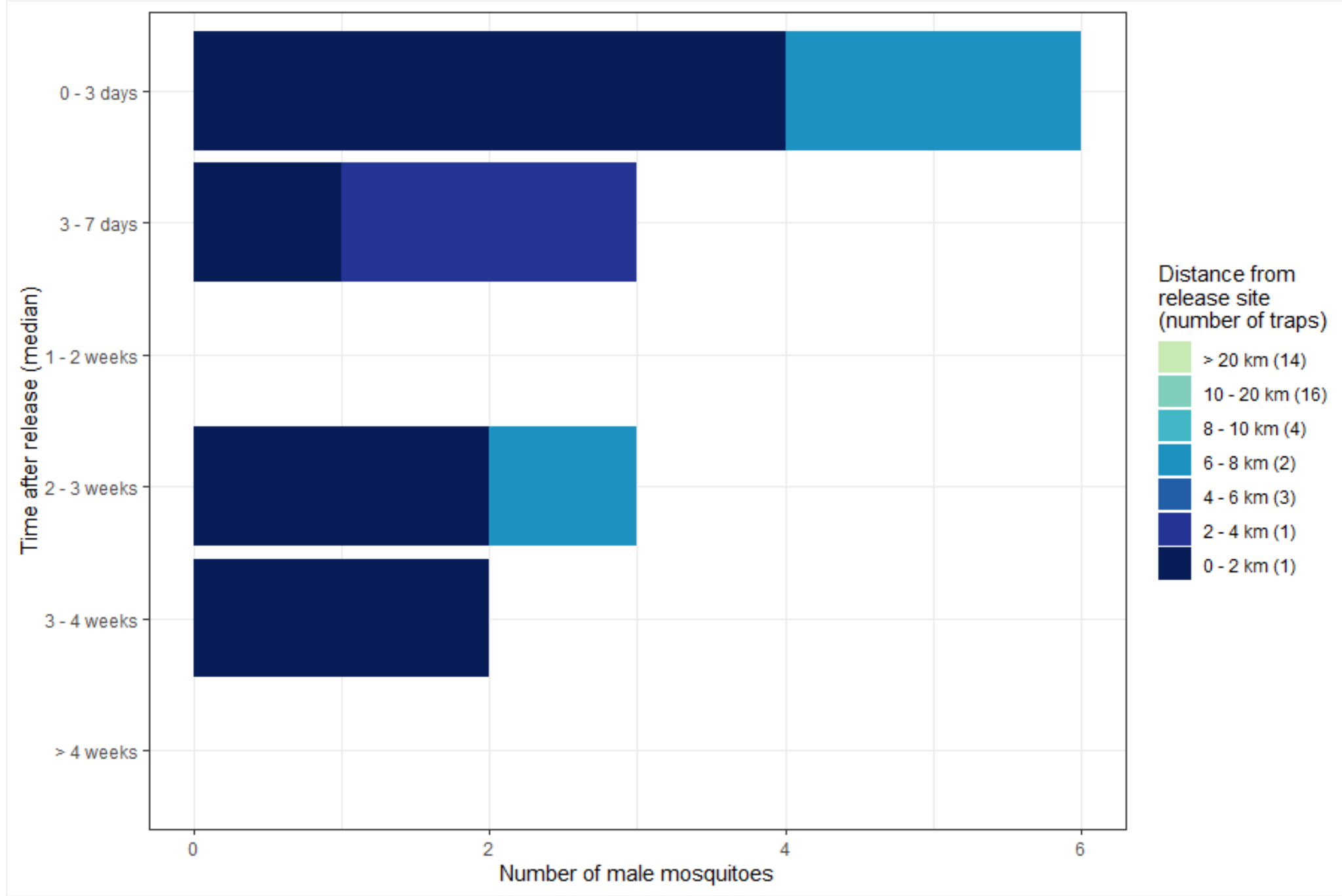

Figure 12. Number of recaptured male mosquitoes blocked by dispersal distance and time interval following release (median days used) in 2016. Number of traps in each distance block is shown in legend. 

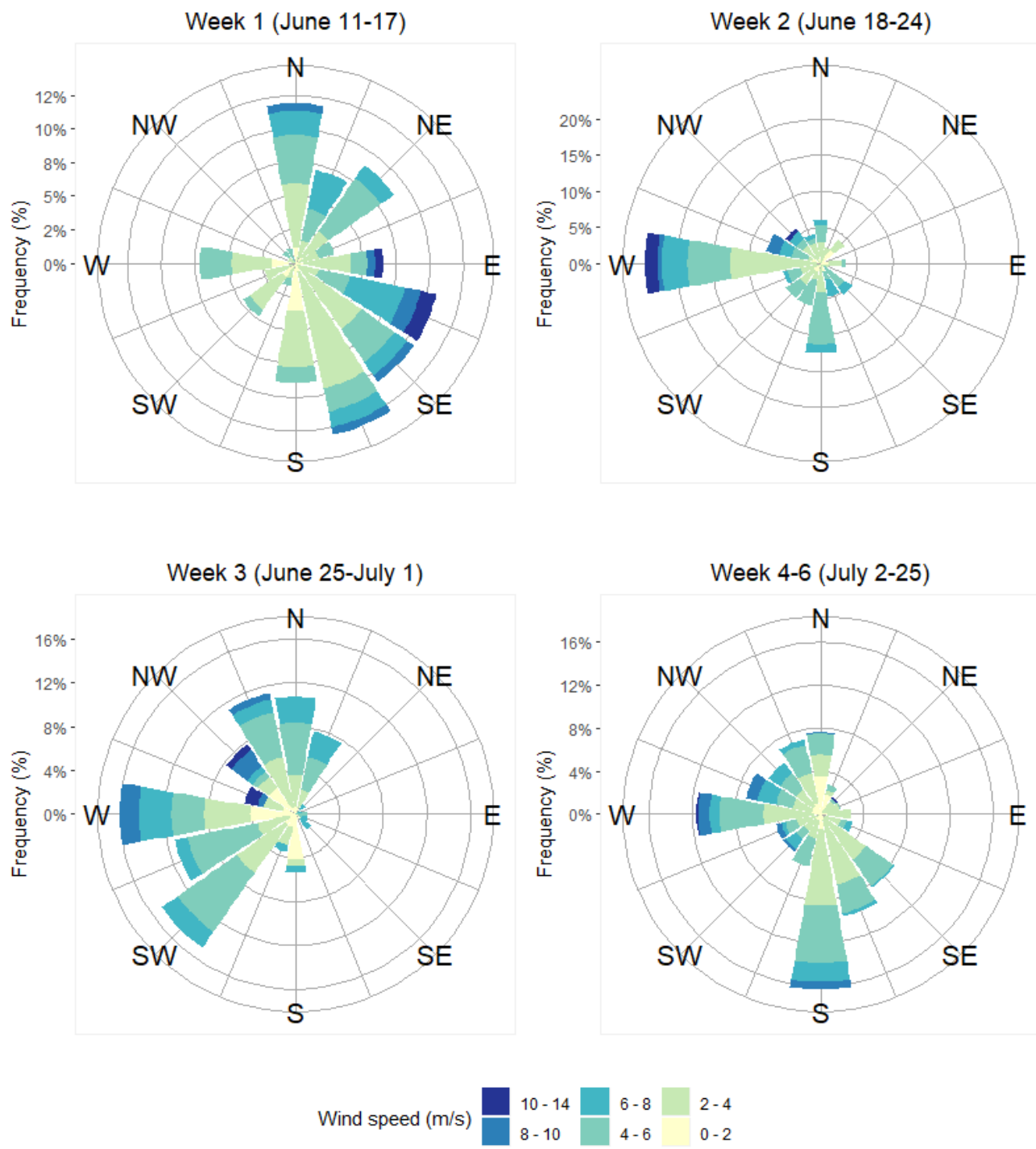

Figure 13. Wind roses for the study period in 2016 (June 11 to July 25), based on hourly wind data from all hours of the day (Environment Canada 2019). The length of radial spokes indicates the frequency of winds blowing from their corresponding direction. 


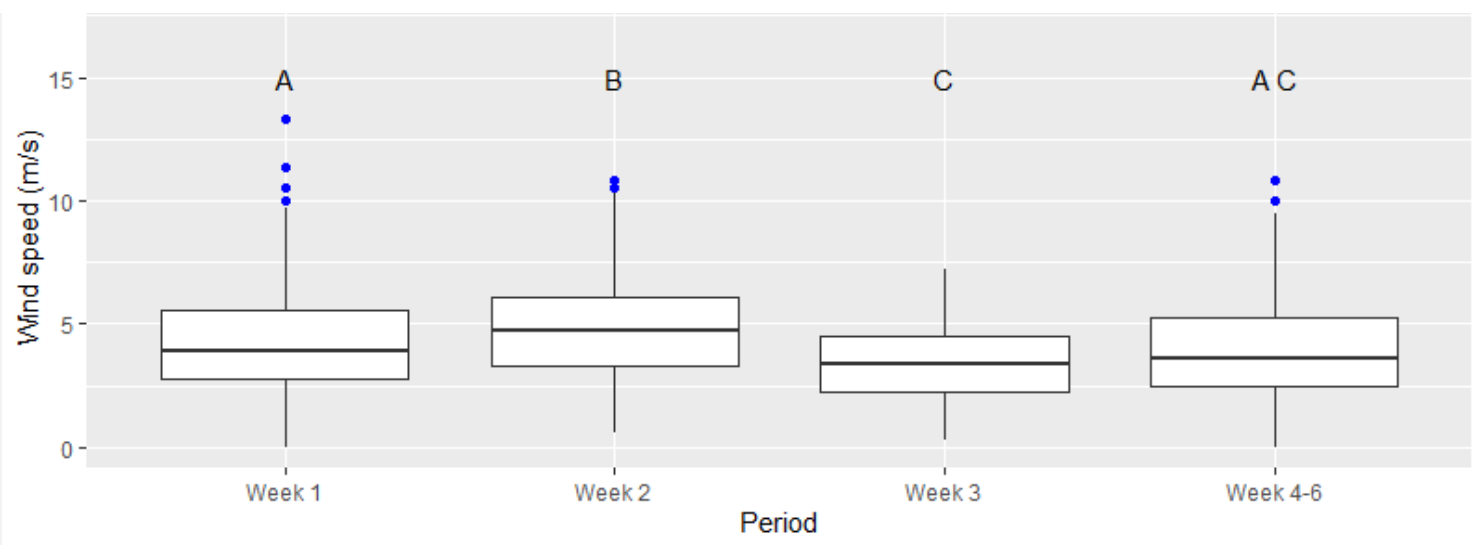

Figure 14. Boxplot comparing wind speeds (measured at $10 \mathrm{~m}$ above ground) between different periods of the 2016 MRR experiment. Outliers are shown in blue. Weekly periods with the same letters do not have significantly different wind speeds $(p>0.05)$.

During week 2 (June 18-24 ${ }^{\text {th }}$ ), wind speed was higher on average in comparison to the other periods (Kruskal-Wallis, $\chi^{2}(3)=43.3, p<0.0001, n=1066$ ).

Lifetime MDT values (see calculation example in Table 7) obtained in 2016 were $8.0 \mathrm{~km}$ for female mosquitoes and $3.7 \mathrm{~km}$ for males (Table 8). Among Ae. vexans, the lifetime MDT for females was $4.6 \mathrm{~km}$, and that for males was similar at $3.8 \mathrm{~km}$. Only Ae. vexans were recaptured within the first seven days of the study period in 2016, so initial flight capabilities were only calculated for this species. The 7-day MDT obtained was 3.9 $\mathrm{km}$ for females and $4.0 \mathrm{~km}$ for males. Too few individuals were recaptured within 3 days following the final release to reliably calculate 3-day MDT values.

Flight ranges were obtained from a regression of the cumulative corrected recapture data against the log-transformed distance $\left(D_{i}\right)$ of the annuli in which they were recaptured. For example, the potential lifetime flight ranges of 50\% (FR 50$)$ and $90 \%\left(F R_{90}\right)$ of female mosquitoes were estimated at $3.2 \mathrm{~km}$ and $15.5 \mathrm{~km}$, respectively (Table 8). These were described by the regression equation of $\log (y)=0.01704 x-0.34232$. Potential lifetime flight ranges for all expected male recaptures were $1.8 \mathrm{~km}$ for $50 \%$, and $7.9 \mathrm{~km}$ for $90 \%$ (Table 8).

When data on recaptured Ae. vexans females were isolated from other recaptures, the potential lifetime $F R_{50}$ value was $2.4 \mathrm{~km}$, and $8.6 \mathrm{~km}$ for the $F R_{90}$ (Table 8, Figure 15). When reduced to only recaptures obtained seven days after the final release, the $F R_{50}$ and 
Table 7. Example of correcting for unequal trap densities and calculating mean distance travelled (MDT) using all recaptured females from 2016, where the coverage correction factor used was 0.406. Distances are in km. Example adapted from Morris et al. 1991.

\section{Annulus}

\begin{tabular}{|c|c|c|c|c|c|c|c|c|c|c|c|c|c|c|c|c|c|}
\hline & 1 & 2 & 3 & 4 & 5 & 6 & 7 & 8 & 9 & 10 & 11 & 12 & 13 & 14 & 15 & 16 & Sum \\
\hline Inner radius $(a)$ & 0.0 & 1.8 & 3.6 & 5.4 & 7.2 & 90. & 10.8 & 12.6 & 14.4 & 16.2 & 18.0 & 19.8 & 21.6 & 23.4 & 25.2 & 27.0 & \\
\hline Outer radius $(b)$ & 1.8 & 3.6 & 5.4 & 7.2 & 9.0 & 10.8 & 12.6 & 14.4 & 16.2 & 18.0 & 19.8 & 21.6 & 23.4 & 25.2 & 27.0 & 28.8 & \\
\hline $\begin{array}{l}\text { Distance } \\
\left(D_{i}\right)=(a+b) / 2\end{array}$ & 0.9 & 2.7 & 4.5 & 6.3 & 8.1 & 9.9 & 11.7 & 13.5 & 15.3 & 17.1 & 18.9 & 20.7 & 22.5 & 24.3 & 26.1 & 27.9 & \\
\hline $\begin{array}{l}\text { Area }\left(\mathrm{km}^{2}\right) \\
=\pi\left(b^{2}-a^{2}\right)\end{array}$ & 10.2 & 30.5 & 50.9 & 71.3 & 91.6 & 112.0 & 132.3 & 152.7 & 173.0 & 193.4 & 213.8 & 234.1 & 254.5 & 274.8 & 295.2 & 315.5 & 2290.2 \\
\hline $\begin{array}{l}\text { corrected for } \\
\text { coverage }\left(A_{i}\right)\end{array}$ & 4.1 & 12.4 & 20.6 & 28.9 & 37.1 & 45.4 & 53.7 & 61.9 & 70.2 & 78.4 & 86.7 & 94.9 & 103.2 & 111.4 & 119.7 & 128.0 & $A_{T}=928.8$ \\
\hline $\begin{array}{l}\text { Number of traps in } \\
A_{i}\left(N_{i}\right)\end{array}$ & 1 & 0.5 & 2.5 & 1 & 4 & 2 & 1 & 4 & 6 & 3 & 2 & 6 & 3 & 4 & 1 & 1 & $N_{T}=41$ \\
\hline$C F_{i}=A_{i} / A_{T} \times N_{t}$ & 0.16 & 0.48 & 0.80 & 1.12 & 1.44 & 1.76 & 2.08 & 2.40 & 2.72 & 3.04 & 3.36 & 3.68 & 4.00 & 4.32 & 4.64 & 4.96 & 41 \\
\hline $\begin{array}{l}\text { Observed recaptures } \\
\left(O R_{i}\right)\end{array}$ & 25 & 10 & 15 & 0 & 12 & 1 & 0 & 0 & 0 & 1 & 0 & 0 & 0 & 0 & 1 & 0 & 65 \\
\hline $\begin{array}{l}\text { Expected recaptures } \\
\left(E R_{i}\right)\end{array}$ & 4 & 10 & 5 & 0 & 4 & 1 & 0 & 0 & 0 & 1 & 0 & 0 & 0 & 0 & 5 & 0 & $E R_{T}=29$ \\
\hline$E R_{T} \times D_{i}$ & 3.6 & 2.59 & 21.6 & 0 & 35.0 & 8.7 & 0 & 0 & 0 & 17.3 & 0 & 0 & 0 & 0 & 121.2 & 0 & 233.5 \\
\hline
\end{tabular}

$29 / 233.5=\mathbf{8 . 0} \mathbf{~ k m}$ - MDT for all female mosquitoes recaptured during the duration of the study period 
Table 8. Summary of analyses to determine mean distance traveled (MDT) and flight ranges $(F R)$ for $50 \%$ and $90 \%$ of recaptures in 2016.

\begin{tabular}{lllccccc}
\hline $\begin{array}{c}\text { Recapture } \\
\text { group }\end{array}$ & Sex & Period & $\begin{array}{c}\text { Observed } \\
\text { recaptures } \\
\text { (OR) }\end{array}$ & $\begin{array}{c}\text { Expected } \\
\text { recaptures } \\
\text { (ER) }\end{array}$ & MDT $(\mathrm{km})$ & $F R_{50}(\mathrm{~km})$ & $F R_{90}(\mathrm{~km})$ \\
\hline All & $\mathrm{F}$ & Lifetime & 65 & 29 & 8.0 & 3.2 & 15.5 \\
Ae. vexans & $\mathrm{F}$ & Lifetime & 55 & 22 & 4.6 & 2.4 & 8.6 \\
Ae. vexans & $\mathrm{F}$ & 7-day & 25 & 13 & 3.9 & 2.3 & 6.7 \\
Ae. vexans & $\mathrm{F}$ & 3-day & 6 & 4 & 3.0 & 1.9 & 3.6 \\
& & & & & & & \\
All & $\mathrm{M}$ & Lifetime & 14 & 4 & 3.7 & 1.8 & 7.9 \\
Ae. vexans & $\mathrm{M}$ & Lifetime & 13 & 4 & 3.8 & 1.7 & 6.8 \\
Ae. vexans & $\mathrm{M}$ & 7-day & 8 & 2 & 4.0 & 1.8 & 7.0 \\
Ae. vexans & $\mathrm{M}$ & 3-day & 2 & $<1$ & $\mathrm{NA}$ & $\mathrm{NA}$ & NA \\
\hline
\end{tabular}

$F R_{90}$ for female Ae. vexans was determined to be $2.3 \mathrm{~km}$ and $6.7 \mathrm{~km}$, respectively (Table 8, Figure 15). Estimates of flight ranges for male Ae. vexans were based on relatively few expected recaptures and were somewhat smaller than female flight ranges (Table 8). In all groups, $F R_{50}$ (median distance travelled) was smaller than the MDT calculated.

\subsection{2 - Mark-release-recapture experiments of 2017}

Across the four releases in 2017 (using different marking dust colours), 28,923 mosquitoes were marked, of which 192 were recaptured. Of these recaptures, 137 (71.4\%) were female and 55 (28.6\%) were male (Table 9). Depending on release date and dust colour, the length of recapture period ranged between 27 and 11 days. The earliest (orange) release had the most successful recapture rate $(2.88 \%)$, and recapture rates declined as shorter releases were carried out later in August.

Unlike in 2016, no significant correlation was found between the number of wild mosquitoes captured and the number of marked mosquitoes recaptured $\left(r_{\tau}=-1.6, p=\right.$ $0.110)$, nor were any correlations found when these data were divided into females $\left(r_{\tau}=-\right.$ $1.8, p=0.080)$ or males $\left(r_{\tau}=1.1, p=0.250\right)$. When divided into the individual releases by colour, the number of marked mosquitoes recaptured and the number of wild mosquitoes 
captured were only correlated for the following groups: 1) males within the first (orange) release $\left.\left(r_{\tau}=3.4, p<0.001\right), 2\right)$ all recaptures within the last (blue) release $\left(r_{\tau}=2.0, p=\right.$ .04937), and 3) males within the last (blue) release $\left(r_{\tau}=2.9, p>.01\right)$. All three of these groups had sample sizes below 20 .

Table 9. Summary of mosquito releases in 2017 by fluorescent marking dust colour. Number of marked mosquitoes was based on manual count. Summary of each individual release (by colour) can be found in Appendix X. The mean recapture rate $\overline{\mathrm{x}}_{\varphi}$ has been calculated with weightings proportional to each release.

\begin{tabular}{|c|c|c|c|c|c|}
\hline Release date & AUGUST 1 & AUGUST 8 & AUGUST 14 & AUGUST 17 & \\
\hline Release no. & 1 & 2 & 3 & 4 & All \\
\hline Marking colour & ORANGE & YELLOW & PINK & BLUE & \\
\hline Total marked & 1752 & 9500 & 5723 & 11948 & 28923 \\
\hline Total recaptured & 49 & 104 & 21 & 18 & 192 \\
\hline$\%$ female & 83.7 & 65.4 & 76.2 & 66.7 & 71.4 \\
\hline$\%$ male & 16.3 & 34.6 & 23.8 & 33.3 & 28.6 \\
\hline Recapture rate $(\%)$ & 2.88 & 1.09 & 0.37 & 0.15 & $\overline{\mathrm{x}}_{\varphi}=\mathbf{1 . 1 6}$ \\
\hline Total trap nights & 27 & 20 & 14 & 11 & \\
\hline
\end{tabular}

Culiseta inornata (Williston, 1893) and Ae. vexans were the most common species recaptured at $38 \%$ and $35 \%$, respectively (Table 10). These proportions did not mirror those seen in the species composition of the wild (unmarked) mosquitoes $(n=2,781)$ collected during the same period. In comparison to the wild mosquitoes, Cs. inornata were overrepresented among the recaptures and Ae. vexans were underrepresented. Eleven (11) species were identified among the recaptures; more than double the number of species identified in the recaptured cohort of 2016.

The ratio of marked mosquitoes to wild mosquitoes in the trap collections was much lower in 2017, at roughly 1 to 47 for males, and 1 to 25 for females. Most traps collected fewer than 200 mosquitoes in total throughout the study period in August, with only trap \#3 collecting more than 500 mosquitoes. Traps that recaptured marked mosquitoes frequently had marked to wild ratios less than 1:10. Trap \#12 only collected 173 wild mosquitoes, but recaptured 45, for a wild-marked ratio of 3:1. 


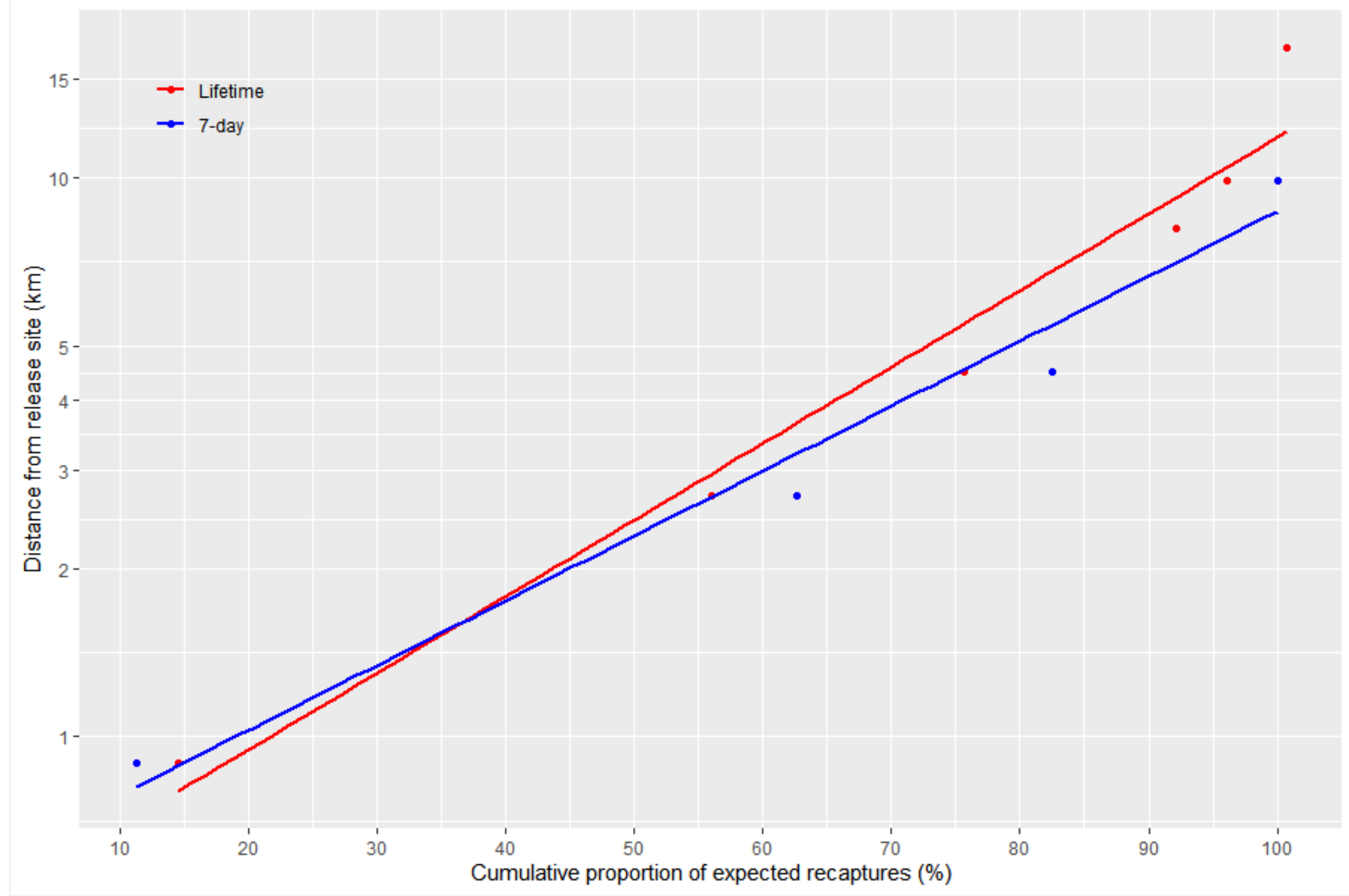

Figure 15. Cumulative percentage of expected female Ae. vexans recaptures $(E R)$ as a function of distance (backtransformed from log-scale) from the release site in 2016. 
Table 10. Species and sex breakdown of recaptured mosquitoes in 2017. Includes composition of wild (unmarked) mosquitoes $(n=2,781)$ for collections identified to species in full for the MRR period (August $1^{\text {st }}$ to August $28^{\text {th }}$ ) for comparison. Percentages are rounded and may not add up to 100 .

\begin{tabular}{|c|c|c|c|c|c|c|c|c|c|c|c|c|c|}
\hline & 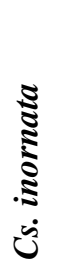 & 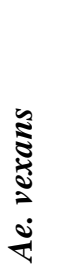 & $\begin{array}{l}\widetilde{\Xi} \\
\bar{\Xi} \\
\dot{\Sigma} \\
\dot{\Sigma}\end{array}$ & 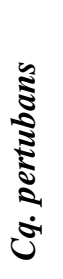 & 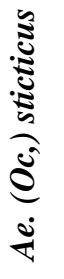 & 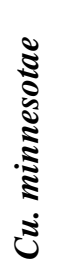 & 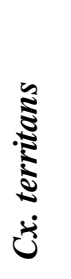 & 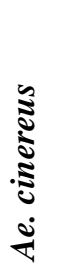 & 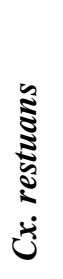 & 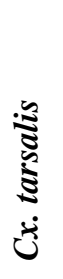 & 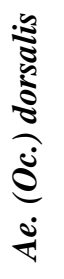 & 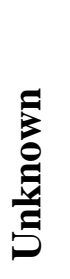 & $\stackrel{\pi}{\stackrel{\pi}{\theta}}$ \\
\hline $\begin{array}{l}\text { Total } \\
\text { recaptured }\end{array}$ & 72 & 67 & 12 & 10 & 9 & 6 & 5 & 3 & 3 & 3 & 1 & 1 & 192 \\
\hline no. females & 69 & 34 & 9 & 5 & 7 & 5 & 2 & 0 & 2 & 3 & 1 & 0 & 137 \\
\hline no. males & 3 & 33 & 3 & 5 & 2 & 1 & 3 & 3 & 1 & 0 & 0 & 1 & 55 \\
\hline $\begin{array}{l}\% \text { of } \\
\text { recaptures }\end{array}$ & 38 & 35 & 6 & 5 & 5 & 3 & 3 & 2 & 2 & 2 & 1 & 1 & \\
\hline $\begin{array}{l}\% \text { of wild } \\
\text { captures }\end{array}$ & 11 & 66 & 3 & 5 & 1 & 1 & 3 & 1 & 4 & 2 & 1 & 1 & \\
\hline
\end{tabular}

Recapture patterns varied temporally and spatially among releases in 2017. The two earliest releases (orange and yellow) were most successful in terms of recapture numbers and rate and most individuals were recaptured in irregular multi-day periods (Figure 16). All releases except for the first (orange) collected the majority (>50\%) of their total recaptures within 7 days of release (Figure 16). More than $85 \%$ of all recaptures were obtained in the first two-week period (up to day 14) following each release, except for the first release (orange), in which more than $25 \%$ of all recaptures were obtained in the $3^{\text {rd }}$ week (days 15 to 21). In all releases except for the last release (blue) no individuals were recaptured after August $22^{\text {nd }}$. Consolidation of standardized recaptures across all releases revealed that over $90 \%$ of all individuals were recaptured within the first two weeks following release (Figure 17). See Appendices X and XI for a daily breakdown of all recaptured mosquitoes.

A total of 19 traps recaptured marked mosquitoes in 2017. Seven of these traps (\#2, $\# 3$, \#5, \#8, \#9, \#11 and \#14) were in areas shaded by trees, whereas the remaining 12 traps (\#1, \#4, \#6, \#7, \#10, \#12, \#13, \#23, \#27, \#28, \#40 and \#51) were all in more open areas 

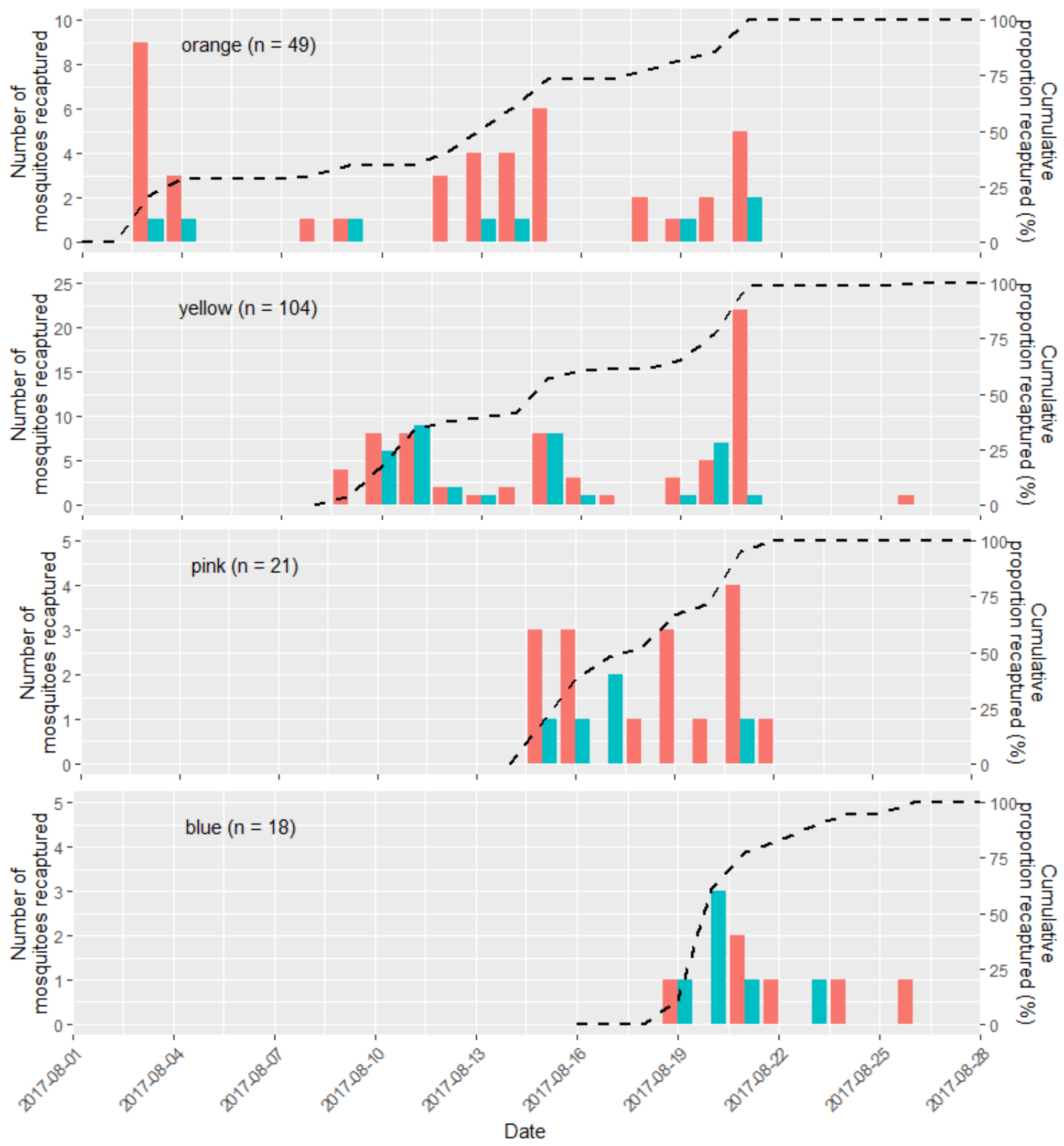

Figure 16. Daily breakdown of recaptured mosquitoes by day and release for 2017, ordered by color listed. Female individuals are indicated by red bars, male individuals are indicated by blue bars. The dashed line indicates the cumulative proportion of mosquitoes recaptured throughout the study period. Releases were on August $1^{\text {st }}, 8^{\text {th }}, 14^{\text {th }}$, and $17^{\text {th }}$. See appendix $\mathrm{X}$ and $\mathrm{XI}$ for raw data. 


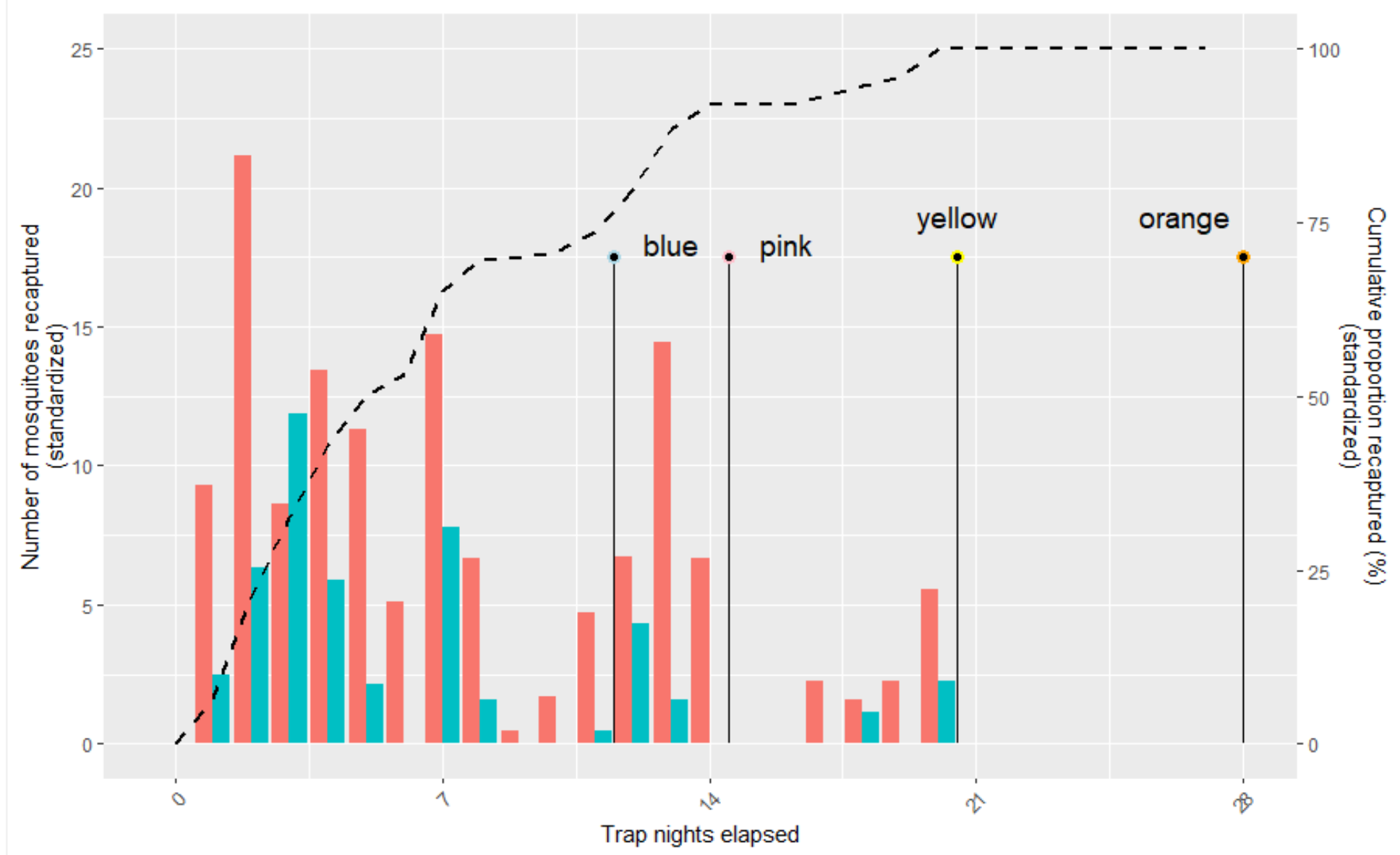

Figure 17. Combined time-series breakdown of recaptured mosquitoes for all releases in 2017 using standardized recapture data from each release. Female individuals are indicated by red bars, male individuals are indicated by blue bars. The dashed line indicates the cumulative proportion of mosquitoes recaptured throughout the study period. Vertical pointers indicate the end of the extent of the monitoring period for individual releases by colour (e.g., markedblue mosquitoes were released last, so were only recaptured for a period of 11 days. See appendix XII for raw data. 
above grassy or herbaceous ground cover (trap \#13 was on bare ground). The trap nearest to the release site (\#1) did not recapture the greatest number of mosquitoes in 2017. Instead, the most recaptures occurred in trap \#12, located $3.7 \mathrm{~km} \mathrm{SSW}$ from the release site, recapturing 45 females (including 38 Cs. inornata specimens) and no males (Figure 18). All release cohorts (distinguished by colour) were represented in this trap's collection in proportions within $5 \%$ of their total proportions among all recaptured individuals. For example, 53\% of mosquitoes recaptured at trap \#12 were marked with yellow dust, and yellow-marked recaptures made up 54\% of all recaptures. The traps in treed areas collected $57 \%$ of all recaptured mosquitoes (110 individuals), including $81 \%$ of all Ae. vexans and $61 \%$ of the Cs. inornata specimens not recaptured in trap \#12. If recaptures from trap \#12 are excluded, then the traps in treed areas collected $75 \%$ of all recaptured mosquitoes, despite representing only a third of the traps that collected marked mosquitoes.

As in 2016, over $95 \%$ of all recaptured mosquitoes were trapped within $8 \mathrm{~km}$ of the release site (Figure 18). Of the seven individuals that dispersed further than $8 \mathrm{~km}$, all were female apart from one male $A e$. vexans recovered at trap \#27 nearly $15 \mathrm{~km}$ from the release site. The maximum dispersal distance observed in the 2017 MRR experiments was 26.4 $\mathrm{km}$ for one female $C q$. perturbans individual, recaptured again in trap \#50 - an observation identical to the record-holder in 2016. See Appendix XIII for a breakdown of recaptured marked mosquitoes by distance from release site.

Trap \#1 captured significantly fewer (3F/0M) mosquitoes than traps \#2 (9F/10M) and \#3 (15F/17M), which are both shaded by trees located within $1 \mathrm{~km}$ of the release site and within $250 \mathrm{~m}$ of a river (Figure 18). Traps \#4 (treed, 6F/1M) and \#5 (grassy, 5F/3M), located respectively 1.3 and $2 \mathrm{~km}$ from the release site but both only $150 \mathrm{~m}$ from the La Salle River, recaptured more than twice as many mosquitoes than trap \#1. As in 2016, trap \#11 (11F/14M), deployed in a private backyard facing the Red River, was among the traps with the most recaptures, being one of only four traps to recapture more than 15 mosquitoes. Of the seven mosquitoes recaptured further than $6 \mathrm{~km}$ from the release site (across five traps, see Figure 19, 20), five of these recaptures were obtained in the three traps (\#27, 28 and 50) that were within 0.25 to $1 \mathrm{~km}$ from a river. 


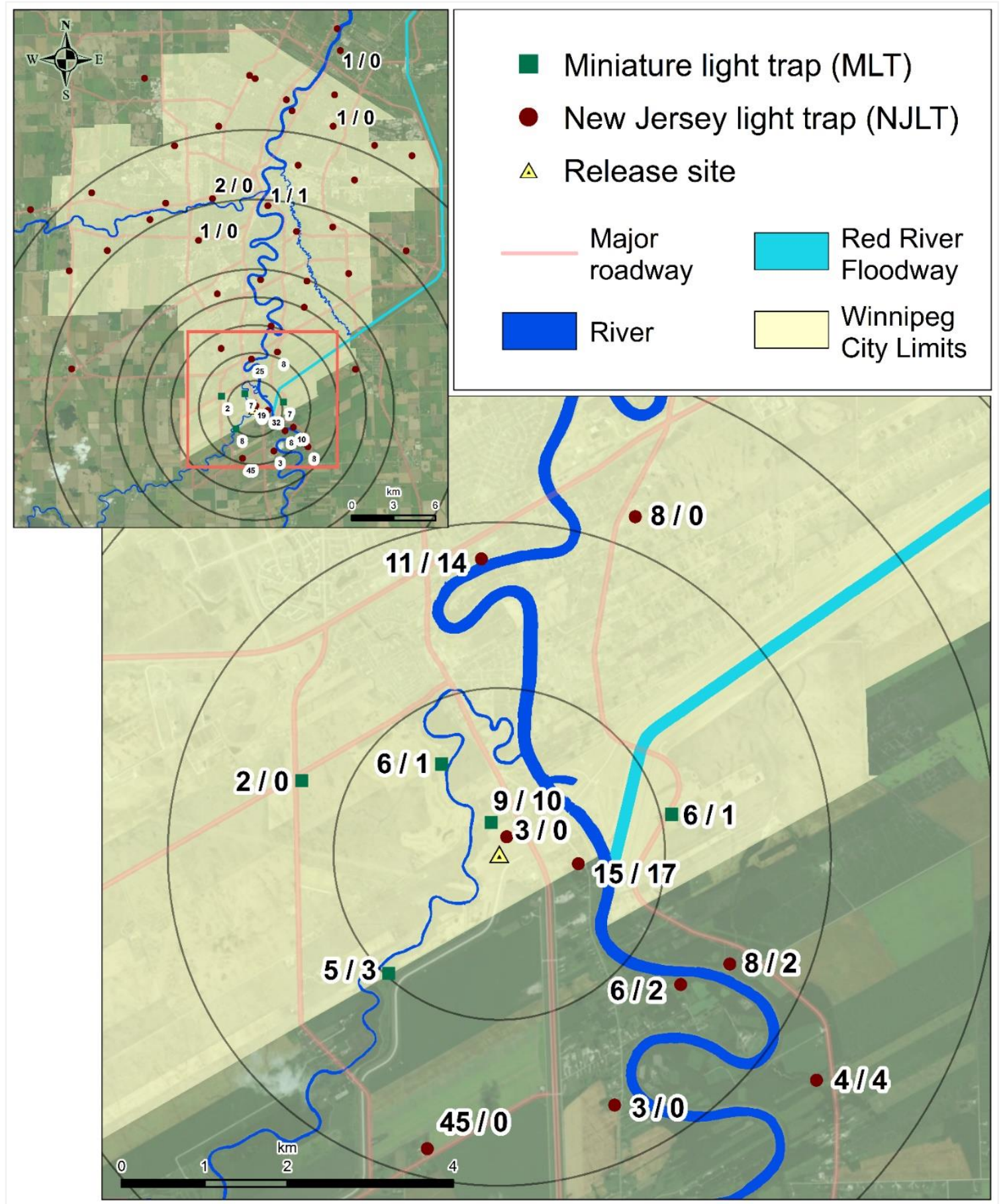

Figure 18. Recovery pattern of recaptured mosquitoes in 2017. Numbers near each trap marker denote the number of marked-recaptured females and males, respectively. Trap markers with no numbers recaptured zero marked mosquitoes. Annuli are separated by 2.0 $\mathrm{km}$ for the first $10 \mathrm{~km}$, then by $5 \mathrm{~km}$ until $20 \mathrm{~km}$. 


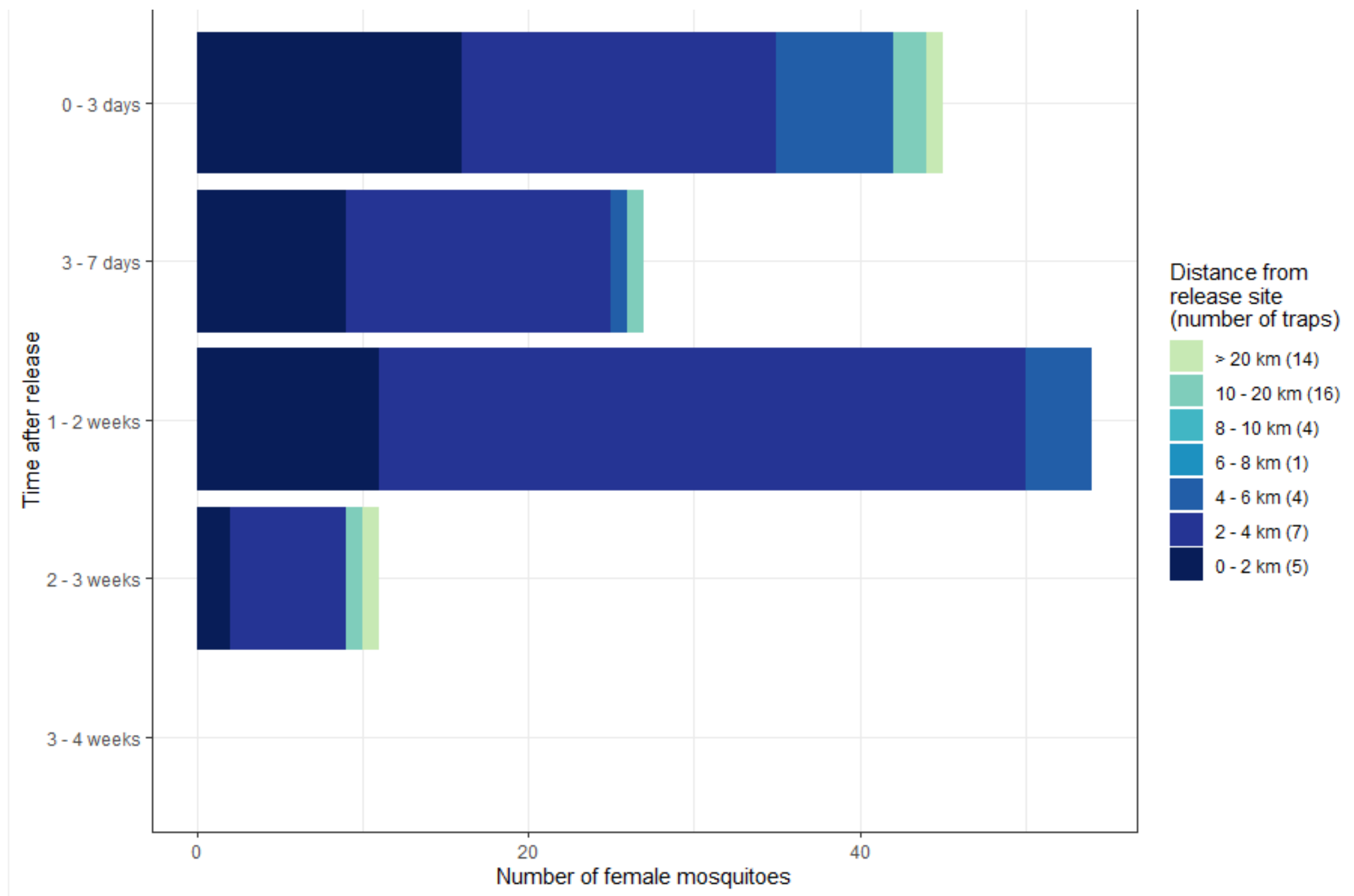

Figure 19. Number of recaptured female mosquitoes blocked by dispersal distance and time interval following release in 2017. Number of traps in each distance block is shown in legend. Note that mosquito counts are not standardized across releases, and cohorts with greater release numbers and longer recapture periods (e.g., yellow, orange) are overrepresented in this figure. 


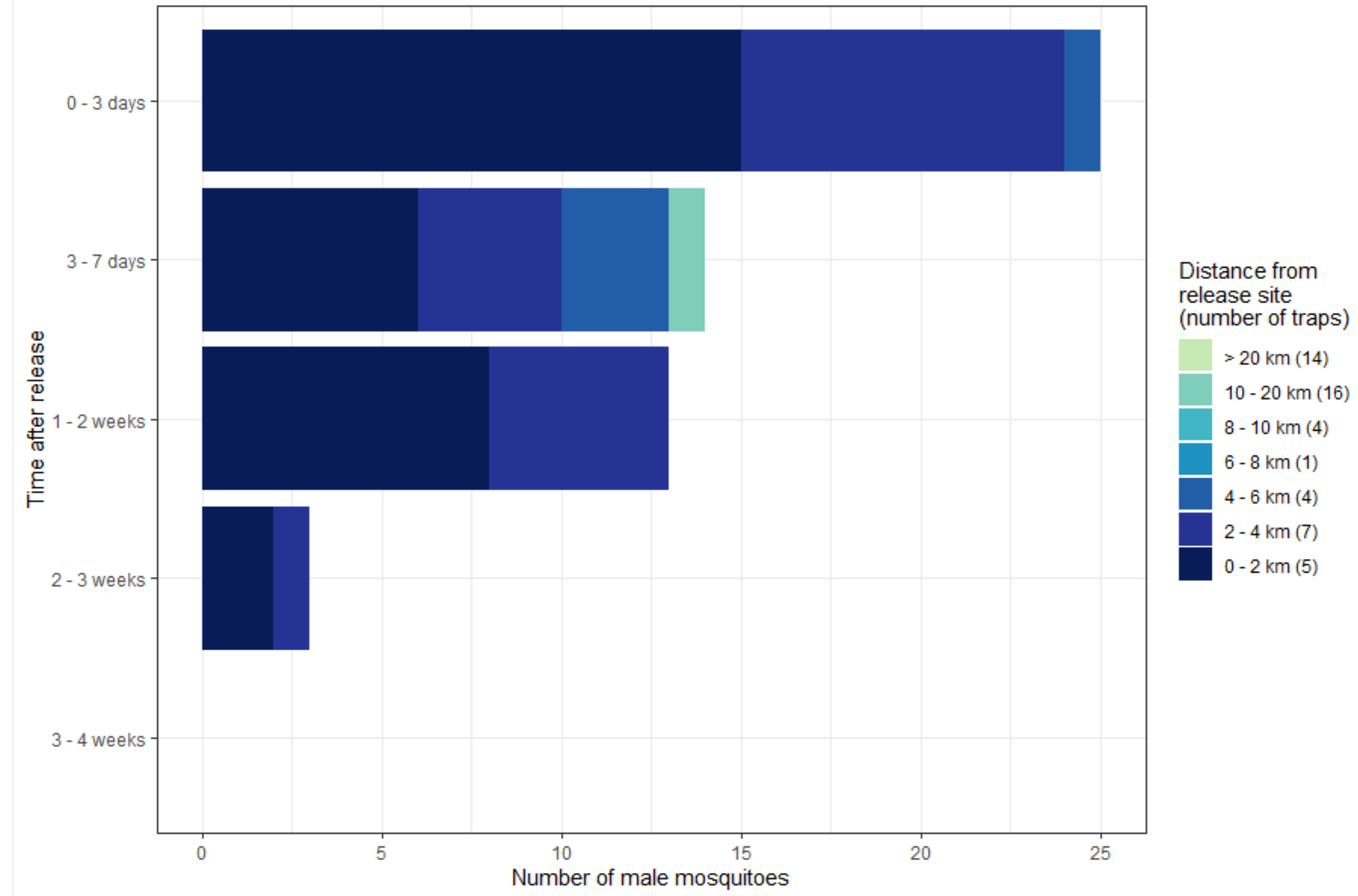

Figure 20. Number of recaptured male mosquitoes blocked by dispersal distance and time interval following release in 2017. Number of traps in each distance block is shown in legend. Note that mosquito counts are not standardized across releases, and the second cohort (yellow) is overrepresented in this figure. 
The remaining two outliers were recaptured in traps \#23 and \#40, each deployed in residential backyards over $2 \mathrm{~km}$ from a river.

The first release cohort (orange) had a total of 49 recaptured mosquitoes (Table 9). Of these, 19 and 16 were identified as Cs. inornata and Ae. vexans, respectively. Within the first three days following release, eight Cs. inornata (all female) were recaptured at an average of $2.9 \mathrm{~km}$ from the release site. The remainder (only one male) were recaptured 13 to 20 days following release, at an average of $3.8 \mathrm{~km}$ from the release site. However, one female specimen was recaptured $12.7 \mathrm{~km}$ (trap \#23) from the release site on day 20. When this outlier was excluded, the average recapture distance observed in Cs. inornata remained at $2.9 \mathrm{~km}$. Nearly half of all Cs. inornata were recaptured at trap \#12, located $3.7 \mathrm{~km}$ south of the release site. Among Ae. vexans recaptures, only three specimens (one male) were recovered within the first week of release (all on day 2), and all were collected in different traps between 3 and $5 \mathrm{~km}$ from the release site. The remaining 13 specimens (three male) were recaptured 8 to 20 days following release, at an average of $3.7 \mathrm{~km}$ from the release site. One female specimen was recaptured $21.0 \mathrm{~km}$ (in trap \#40) from the release site on day 20. When this outlier was excluded, the average recapture distance was $2.6 \mathrm{~km}$ for all recaptured Ae. vexans. Recaptures of Ae. vexans were not concentrated at any trap in this cohort.

A total of 104 marked mosquitoes were recovered from the second release cohort marked with yellow (Table 9), of which 32 specimens were identified as Cs. inornata and 42 as Ae. vexans. All recaptured Cs. inornata were female, and only five were recaptured within the first three days following release, at traps located an average of $2.7 \mathrm{~km}$ from the release site. The remaining 27 specimens were recaptured 6 to 13 days after release, and 18 of these were recaptured on day 13 at trap \#12, $3.7 \mathrm{~km}$ from the release site. Of the recaptured Aedes vexans in the yellow cohort, over half (23 specimens) were male, but recapture trends were similar when compared to females. Within the first 3 days following release, $20 \mathrm{Ae}$. vexans specimens were recaptured at an average of $2.6 \mathrm{~km}$ from the release site, or $1.9 \mathrm{~km}$ when a female outlier recaptured at $15.4 \mathrm{~km}$ (trap \#28) on day 3 was excluded. The remainder were recaptured 4 to 13 days post-release and were collected an average of $2.0 \mathrm{~km}$ from the release site. Nearly a quarter of Ae. vexans (9 specimens) 
recaptured from this cohort were collected at trap \#2 (400 $\mathrm{m}$ from the release site). Another quarter (11 specimens) were recaptured at trap \#3 (1 $\mathrm{km}$ from the release site). Additionally, two other females from this release were collected further than $10 \mathrm{~km}$ from the release site. These included one $C x$. restuans, collected on day 3 at trap \#28 with the outlier Ae. vexans above, and the Cq. perturbans specimen at trap \#50, located $26.4 \mathrm{~km}$ from the release site and recovered only one day following release.

Only 21 marked specimens were recovered from the third release cohort (pink) including 10 Cs. inornata (1 male) and four Ae. vexans (two males, two females). Nearly half of all specimens (10) were recaptured in the first three days, with the remainder having been recaptured after four to nine days following release. All recaptured Cs. inornata were collected at least four days post-release, at traps located an average of $2.7 \mathrm{~km}$ from the release site. Six Cs. inornata specimens were collected from trap \#12, located $3.7 \mathrm{~km}$ from the release site. There were no recaptures in this cohort collected further than trap \#12. Among Ae. vexans, recaptured adults in this cohort were evenly split between traps \#3 and $\# 11,1.0$ and $3.6 \mathrm{~km}$ from the release site, respectively.

The results of the fourth release cohort (blue) were similar to the third: of 18 recaptured specimens, 11 were Cs. inornata (one male) and 5 were Ae. vexans (four males). Over half of all specimens (11) were recaptured in the first three days, with the remainder having been recaptured after 5 to 10 days following release. Six Cs. inornata specimens were recovered within three days of release, at traps located an average of $2.5 \mathrm{~km}$ from the release site. Those Cs. inornata recaptured after this period were collected at traps located an average of $4.7 \mathrm{~km}$ from the release site, though one of them was a female outlier recovered on day 5 at trap \#27, located $14.6 \mathrm{~km}$ from the release site. Similarly, the average distance for recovery of Ae. vexans in this cohort was $5.3 \mathrm{~km}$, though an outlier was recaptured on day 7 at the same trap as the outlier described previously. See Appendix XIV for a complete list of all recaptured mosquitoes, recapture dates and trap locations.

The prevailing wind direction and speed during the study period in 2017 was variable (Figure 21). Winds during week 1 of the first (orange) release (August 1 to 7) originated from the west and northerly direction. Relatively weak winds from the NNE were observed during the week 2 (August 8 to 14), when the second (yellow) release was 

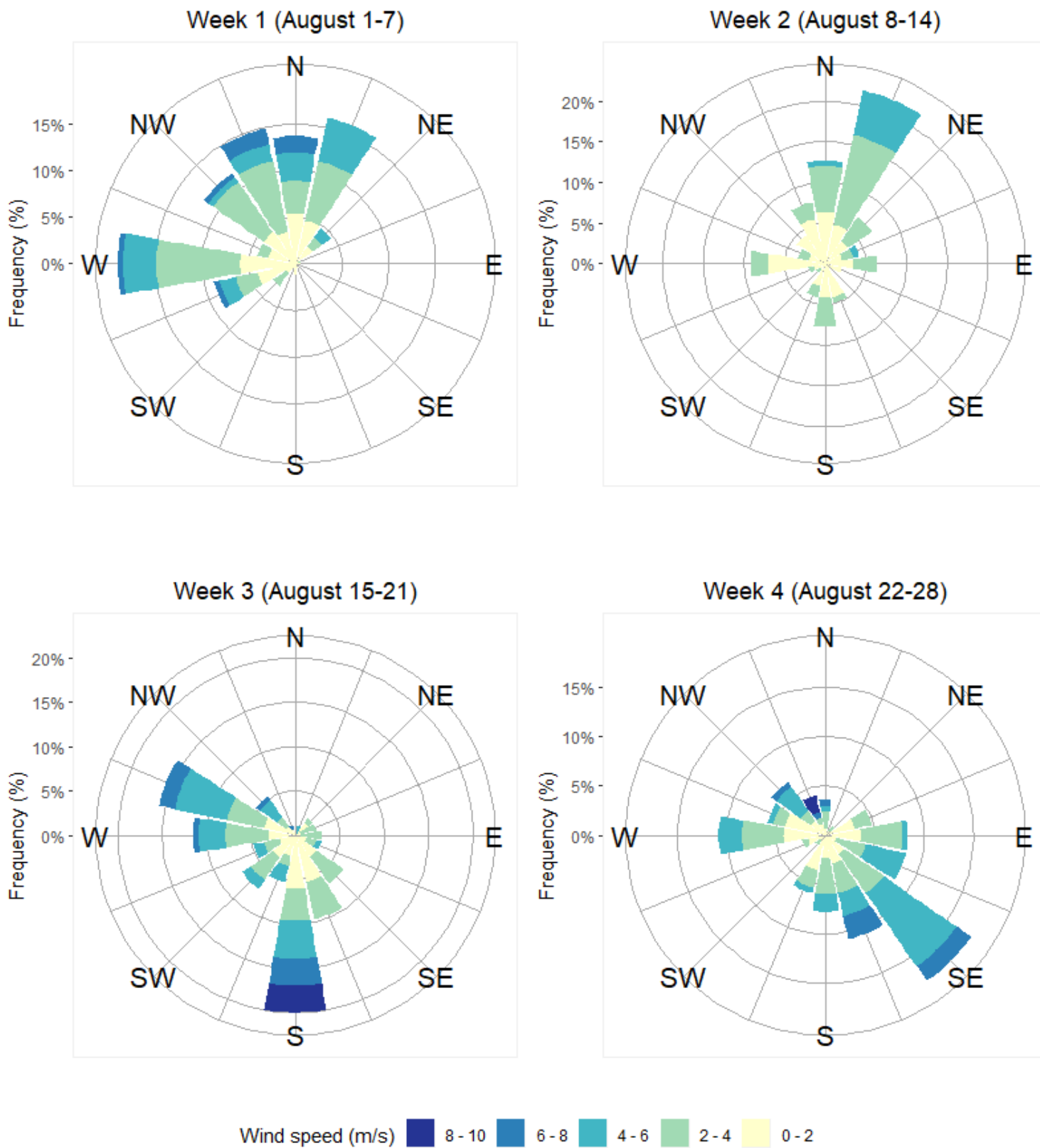

Figure 21. Wind roses for the study period in 2017 (August 1 to 28), based on hourly wind data from all hours of the day (Environment Canada 2019). The length of radial spokes indicates the frequency of winds blowing from their corresponding cardinal direction. 
initiated. There were strong southerly winds and winds from the west during week 3 (August 15 to 21), which gave way to predominantly southeasterly winds during the final week of the study (August 22 to 28). The average wind speed throughout the study period was lower than in 2016, at $2.9 \mathrm{~m} / \mathrm{s}(\mathrm{SD}=1.8)$. There were significant differences in wind speed between time periods (Figure 22). During week 2, wind speed was higher on average in comparison to the other periods (Kruskal-Wallis, $\chi^{2}(3)=40.4, \mathrm{p}<0.0001, n=642$ ).

Figure 22. Boxplot comparing wind speeds (measured at $10 \mathrm{~m}$ above ground) between different periods of the 2017 MRR experiment. Outliers are shown in blue. Weekly periods with the same letters do not have significantly different wind speeds ( $p>0.05$ ).

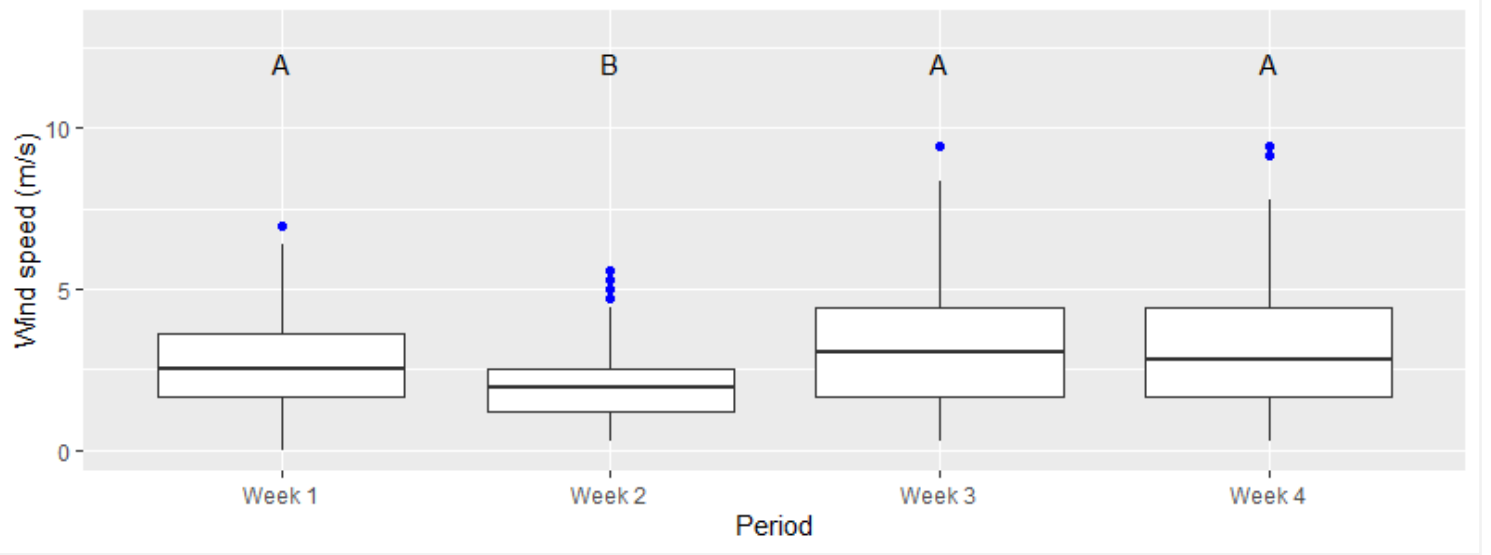

Using $1.8 \mathrm{~km}$ annuli, the lifetime MDT calculated for mosquitoes of all species combined in the 2017 studies was $7.6 \mathrm{~km}$ for females and $4.3 \mathrm{~km}$ for males (Table 11). The lifetime $\mathrm{FR}_{50}$ and $\mathrm{FR}_{90}$ of all females was $1.5 \mathrm{~km}$ and $3.7 \mathrm{~km}$, respectively. For Ae. vexans, females had a lifetime MDT of 7.5 km and a 7-day MDT value of $5.8 \mathrm{~km}$. Similarly, a 7day MDT of $5.7 \mathrm{~km}$ was calculated for male Ae. vexans recaptures, with a lifetime MDT of $5 \mathrm{~km}$. The $F R_{50}$ for female Ae. vexans fell between 3.4 and $4.4 \mathrm{~km}$, depending on the time interval (Table 11). The median flight range $\left(F R_{50}\right)$ for male Ae. vexans fell between 2.4 and $4.4 \mathrm{~km}$. The $F R_{90}$ values determined for female Ae. vexans were over $14 \mathrm{~km}$. Male $A$ e. vexans varied in their $F R_{90}$, ranging from 5.1 to $16.1 \mathrm{~km}$ depending on the time interval.

Too few male Cs. inornata were recaptured to perform analyses, but enough female Cs. inornata were recovered to estimate their lifetime MDT at $5.2 \mathrm{~km}$, with a similar 7-day MDT of $5.3 \mathrm{~km}$ (Table 11). Their median flight range was determined to be between 1.8 and $2.7 \mathrm{~km}$, whereas their $F R_{90}$ values were between 3.2 and $6.4 \mathrm{~km}$. MDT and FR values 
Table 11. Summary of analyses to determine mean distance traveled (MDT) and flight ranges (FR) for 50\% and $90 \%$ of recaptures in 2017 , calculated using $1.8 \mathrm{~km}$ annuli with full data. Only groups with $\geq 10$ observed recaptures were included.

\begin{tabular}{|c|c|c|c|c|c|c|c|}
\hline Recapture group & Sex & Period & $\begin{array}{c}\text { Observed } \\
\text { recaptures }(\mathrm{OR})\end{array}$ & $\begin{array}{c}\text { Expected } \\
\text { recaptures }(\mathbf{E R})\end{array}$ & MDT (km) & $F R_{50}(\mathrm{~km})$ & $F R_{90}(\mathrm{~km})$ \\
\hline All & $\mathrm{F}$ & Lifetime & 137 & 95 & 7.6 & 1.5 & 3.7 \\
\hline All & $\mathrm{F}$ & 7-day & 71 & 12 & 7.7 & 4.6 & 15.9 \\
\hline All & $\mathrm{F}$ & 3-day & 45 & 12 & 15.1 & 5.2 & 20.0 \\
\hline Cs. inornata & $\mathrm{F}$ & Lifetime & 69 & 13 & 5.2 & 2.4 & 5.7 \\
\hline Cs. inornata & $\mathrm{F}$ & 7-day & 30 & 5 & 5.3 & 2.7 & 6.4 \\
\hline Cs. inornata & $\mathrm{F}$ & 3-day & 18 & 3 & 4.0 & 1.8 & 3.2 \\
\hline Ae. vexans & $\mathrm{F}$ & Lifetime & 34 & 5 & 7.5 & 3.4 & 14.1 \\
\hline Ae. vexans & $\mathrm{F}$ & 7-day & 21 & 3 & 5.8 & 4.2 & 14.3 \\
\hline Ae. vexans & $\mathrm{F}$ & 3-day & 15 & 2 & 6.6 & 4.4 & 15.2 \\
\hline All & M & Lifetime & 55 & 6 & 4.3 & 4.4 & 15.8 \\
\hline All & M & 7-day & 39 & 4 & 4.7 & 4.1 & 13.9 \\
\hline All & M & 3-day & 25 & 2 & 3.0 & 2.6 & 6.3 \\
\hline Ae. vexans & M & Lifetime & 33 & 4 & 5.0 & 4.4 & 16.1 \\
\hline Ae. vexans & M & 7-day & 22 & 3 & 5.7 & 4.1 & 13.6 \\
\hline Ae. vexans & M & 3-day & 15 & 2 & 3.3 & 2.4 & 5.1 \\
\hline
\end{tabular}


Table 12. Summary of analyses to determine mean distance traveled (MDT) and flight ranges ( $F R$ ) for $50 \%$ and $90 \%$ of recaptures in 2017 , calculated using $0.5 \mathrm{~km}$ annuli with only recaptures beyond $5 \mathrm{~km}(\mathrm{n}=7)$ removed. Only groups with $\geq 10$ observed recaptures were included.

\begin{tabular}{|c|c|c|c|c|c|c|c|}
\hline Recapture group & Sex & Period & $\begin{array}{c}\text { Observed } \\
\text { recaptures }(\mathrm{OR})\end{array}$ & $\begin{array}{c}\text { Expected } \\
\text { recaptures (ER) }\end{array}$ & MDT (km) & $F R_{50}(\mathrm{~km})$ & $F_{R 90}(\mathrm{~km})$ \\
\hline All & $\mathrm{F}$ & Lifetime & 131 & 455 & 3.3 & 2.5 & 5.1 \\
\hline All & $\mathrm{F}$ & 7-day & 67 & 231 & 3.2 & 2.7 & 6.1 \\
\hline All & $\mathrm{F}$ & 3-day & 42 & 154 & 3.3 & 2.8 & 6.6 \\
\hline Cs. inornata & $\mathrm{F}$ & Lifetime & 67 & 253 & 3.5 & 2.1 & 4.0 \\
\hline Cs. inornata & $\mathrm{F}$ & 7-day & 29 & 112 & 3.4 & 2.5 & 5.4 \\
\hline Cs. inornata & $\mathrm{F}$ & 3-day & 18 & 75 & 3.4 & 2.7 & 6.0 \\
\hline Ae. vexans & $\mathrm{F}$ & Lifetime & 32 & 98 & 2.9 & 3.0 & 7.9 \\
\hline Ae. vexans & $\mathrm{F}$ & 7-day & 20 & 63 & 2.9 & 3.4 & 10.0 \\
\hline Ae. vexans & $\mathrm{F}$ & 3-day & 14 & 41 & 2.8 & 3.8 & 12.6 \\
\hline All & M & Lifetime & 54 & 148 & 2.7 & 3.4 & 10.4 \\
\hline All & M & 7-day & 38 & 119 & 2.7 & 3.7 & 11.7 \\
\hline All & M & 3-day & 25 & 77 & 2.5 & 3.4 & 10.6 \\
\hline Ae. vexans & M & Lifetime & 32 & 84 & 2.8 & 3.2 & 9.4 \\
\hline Ae. vexans & M & 7-day & 21 & 67 & 2.8 & 3.3 & 9.8 \\
\hline Ae. vexans & M & 3-day & 15 & 51 & 2.8 & 3.2 & 8.9 \\
\hline
\end{tabular}


from the 3-day period varied across all species and sexes, with values ranging from 3.2 to $20 \mathrm{~km}$.

When MDT and FR were calculated using $0.5 \mathrm{~km}$ annuli, with the seven recaptures observed beyond $5 \mathrm{~km}$ eliminated, the MDT range for all the above groups was narrowed to values between 2.5 and $3.5 \mathrm{~km}$ (Table 12). The MDT values of female and male Ae. vexans obtained with this procedure were nearly identical regardless of time interval. Median flight ranges followed similar trends across all species, with $\mathrm{FR}_{50}$ values between 2.1 and $3.8 \mathrm{~km}$. The FR 90 values determined were variable, but smaller in range (between 4.0 and $12.6 \mathrm{~km}$ ) in comparison to those calculated with $1.8 \mathrm{~km}$ annuli.

\section{4 - Discussion}

In 2016, fewer traps (only four) were operated within $5 \mathrm{~km}$ of the release site compared to 2017, with the intention for the experiment to function as a proof of concept. Mark-release-recapture experiments that seek to measure dispersal in excess of a few kilometers often suffer from very low recapture rates (Brust 1980; Bogojević et al. 2011). Therefore, the study in 2016 was designed to establish whether long distance dispersal by adult mosquitoes into urban areas could be adequately detected by an MRR experiment in this setting. The results from the 2016 MRR indicated that the procedure was suitable to measure dispersal, and so more traps were included in 2017. The experiments in 2017 were intended to increase precision of flight distance estimates and observe the directional dynamics of dispersal behaviour, and therefore included 10 more traps in the $5 \mathrm{~km}$ radius near the release site. As larvae, mosquitoes were obtained from different sources, and reared and released at different periods in the summer. This led to a difference in the species composition of marked mosquitoes between years, where in 2016 the floodplain mosquito Ae. vexans was most abundant among recaptures. While Ae. vexans was still present in appreciable numbers among the 2017 recaptures, the manner of procuring larvae from an artificial container habitat led to a greater diversity of mosquitoes being marked. As such, the results from these mark-release-recapture experiments between years cannot be directly compared and the implications of their findings will be discussed separately. 
Finally, interpreting dispersal movements near urban centers with respect to wind should be done with caution as wind diversions created by buildings and other infrastructure may also muddle the influence of wind velocity. Therefore, in the present study, the variability in wind velocity and the possible role of wind in noteworthy recapture events shall be considered on a short-term basis later in the discussion, acknowledging the limitations of using wind data collected roughly $20 \mathrm{~km}$ from the release site.

\section{Dispersal behaviour}

Results from both years suggest that many Ae. vexans females fly upwards of $3 \mathrm{~km}$ within a few days following emergence, and have a lifetime flight range of at least $8 \mathrm{~km}$, possibly up to $15 \mathrm{~km}$. These results were generally in agreement with Brust's (1980) work on Ae. vexans in the same general area in 1976 and 1977, where adults flew to the edge of the 8 to $11 \mathrm{~km}$ trapping radius within three to five nights. Estimates of mean and maximum flight distances by all other literature with a focus on Ae. vexans dispersal (Table 1) were either surpassed (due to a limited trapping extent in these studies), or reasonably met within the range of several kilometers.

While usually not addressed by others, we can also speculate from this study that males may also make long flights of distances matching those of females. Adults may have already mated at least once while confined to their rearing enclosures prior to release. It follows that males that can trail females towards other oviposition, feeding or resting sites would have a reproductive advantage by way of increased encounters with other newly emerged females at these locations. This adaptation would be more pronounced in urban or peri-urban areas like Winnipeg, where mosquito habitats are scattered across longer distances with less connectivity.

In 2017, over half $(n=38)$ of the marked female Cs. inornata recaptured were observed at a single trap (\#12) located $3.7 \mathrm{~km}$ south of the release site. The reported MDT values for Cs. inornata are heavily biased toward this trap, which was located on a small farm with a several dozen animals, mostly sheep and horses. Since all Cs. inornata collected at this location were female, they were likely attracted by the odours produced by these animals. The distance covered in this dispersal, coupled with the lack of tree or 
forest shelter present in a direct path from the release site, suggests that female Cs. inornata would have needed to either fly upwind to locate these hosts, or to have used a sheltered corridor. Dispersal within a sheltered corridor, such as the trees surrounding the banks of the La Salle River (1.3 km away from trap \#12) or Red River (2.5 km away from trap \#12), would decrease the risk of desiccation than if they were to travel in open areas.

Indeed, Cs. inornata made up half of the eight recaptures obtained from the trap located near La Salle (\#5), which was equidistant from both trap \#12 and the release site. An analysis of wind velocity trends in the 24 hours prior to each recapture event at trap $\# 12$, indicated that both upwind flight and use of the La Salle as a corridor are plausible. Winds originating from either the south or west (often both) were most common prior to over $85 \%$ of recapture events, whereas winds originating from the north, which could have carried the mosquitoes passively, were only present prior to less than $20 \%$ of Cs. inornata recapture occurrences at this trap, and at average speeds $(\sim 2.5 \mathrm{~m} / \mathrm{s})$. During the period of August $20^{\text {th }}$ and $21^{\text {st }}$, in which 28 female Cs. inornata were recaptured at trap \#12, strong westerly winds (>5 m/s) could have carried them away from their refuge in the foliage near La Salle. Relatively weaker $(\sim 2.5 \mathrm{~m} / \mathrm{s})$ winds from the south and south-west that followed could have allowed them to detect host emanations within a close enough range for them to modulate their navigation upwind. Strong south winds exceeding $5 \mathrm{~m} / \mathrm{s}$ on August $19^{\text {th }}$ and $20^{\text {th }}$ may have also contributed to the collection of two Cs. inornata in traps \#23 and \#27 on August $21^{\text {st }}$.

Insights on Cs. inornata from the 2017 studies revealed more about their host preference rather than dispersal ability. Nevertheless, this species' ability to disperse several kilometers in the first few days following emergence was demonstrated.

Too few Cq. perturbans were recaptured to allow for precise analysis and discussion of their dispersal capabilities. However, it is noteworthy that during both years, a single marked individual appeared in trap \#50, $26.4 \mathrm{~km}$ north from the release site. Contamination at this location is unlikely, given that the pre-release monitoring found no false positives and that the specimens were marked with different dust colours. While the 2016 specimen appeared 11 days following the final release and may have made this journey at an unremarkable pace, the 2017 far-reaching specimen was recovered only one 
day following its release. The climate record's data for the evening preceding this recapture on August $9^{\text {th }}$ show winds originating from a generally east or north-easterly direction, at speeds of up to $8 \mathrm{~m} / \mathrm{s}$. It is possible from this observation that $C q$. perturbans can fly upwind against moderate breezes. We can also speculate from the other $C q$. perturbans recaptured in these studies that they are competent long-distance fliers, commonly with flight ranges exceeding $3 \mathrm{~km}$, supporting the findings of Horsfall (1955), and Verdonschot and Besse-Lototskaya (2014). To the best of our knowledge, this is the first MRR experiment to measure dispersal distances in Cs. inornata or Cq. perturbans.

\section{Male mosquitoes}

It is also worth noting that many male mosquitoes of different species were recaptured more than 10 days following their release, which is commonly reported to be the upper lifespan limit for a male mosquito. However, results from both years suggest that this may not be true for all mosquitoes and that some male mosquito species in northern temperate regions may survive longer than those in southern latitudes. There is some precedent for this claim among other MRR studies (Stage et al. 1937). In 2016, there were 5 male specimens (4 Ae. vexans, 1 unknown) recaptured either 19 or 23 median days following their release (specimens 58, 59 and 68, 74 75, respectively; see Appendix IX). Three of these specimens were recaptured at the trap closest to the release site, but the other two were found at traps either 3.6 (\#11) or $7.8 \mathrm{~km}$ (\#52) from this point.

An additional 14 long-lived male specimens were observed in 2017. All were recaptured 10 to 20 days following their release, at various distances up to $3.6 \mathrm{~km}$. Among these were ten male Ae. vexans individuals marked with either yellow or orange dusts, all with only one or two visible dust particles on variable body parts including the wing, abdomen, and tibiae. Other species also observed to have apparently long-lived males in 2017 were Cx. restuans, Cs. inornata, Anopheles earlei Vargas, 1943 and Aedes cinereus Meigen, 1818, with one individual each. Notably, the single male $C x$. restuans specimen (\#133; see Appendix XIV) had extensive dust coverage (>100 particles) and had been recaptured $400 \mathrm{~m}$ from the release site (trap \#2) after 12 days following release. The dust coverage was unusual (Figure 23) and suggested that the individual may have been marked 
Figure 23. Images of three marked mosquitoes taken with a microscope in dark-field equipped with a UV light source. Each indicates the extent of dust coverage (by number of visible dust particles). Numbers correspond to a specimen IDs in Appendix XIV.
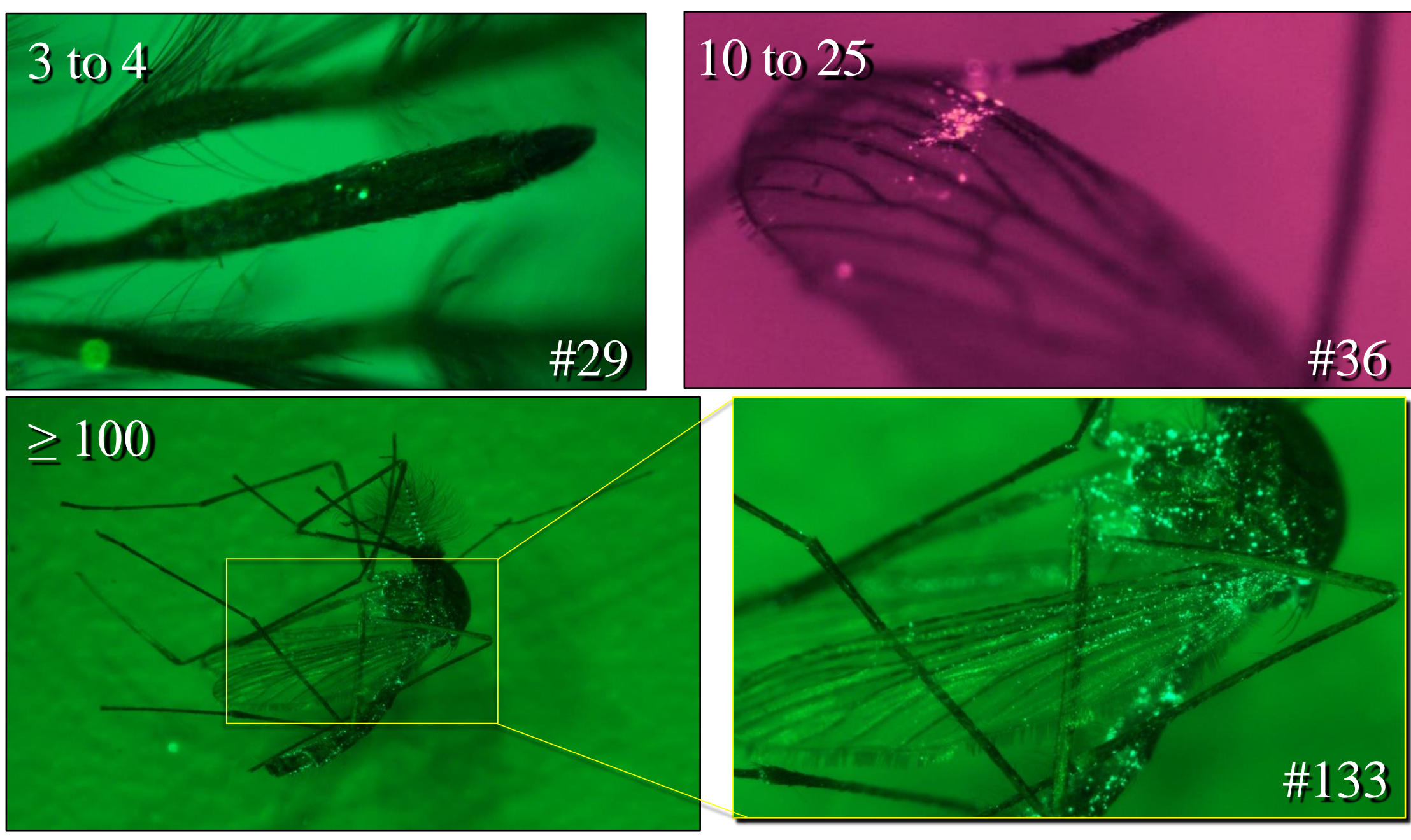

$\geq 100$

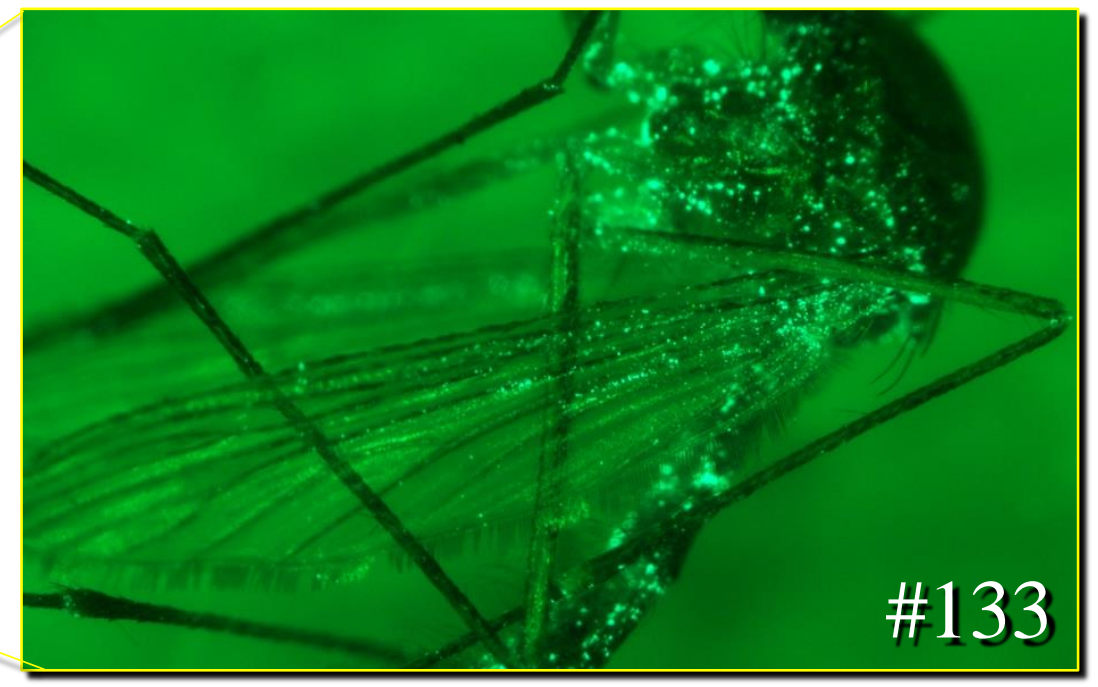


more recently than 12 days prior, assuming that most marks are lost in flight in the days following release.

Indeed, out of all mosquito specimens recaptured in 2017 regardless of species or sex, only eight had $\geq 10$ dust particles visible on their bodies. Of these eight, all were recaptured within $3 \mathrm{~km}$ of the release site and five of them were recaptured less than 3 days following release. The only other mosquito in the 2017 dataset with extensive dust coverage comparable to the male $C x$. restuans individual named above was another male (Cs. inornata) recaptured in the same trap (\#2) only 3 days following release. A look at patterns in dust coverage (Table 13) reveals a clear trend: On average, recaptured specimens with 3 or more visible dust particles were more likely to be male than the average recaptured individual and tended to be recaptured closer to the release site.

This could suggest that males remained at their site of emergence longer than females on average, which allowed them to be remarked more than once by possible residual dust on the surrounding vegetation in the days after release. Slow movement away from breeding areas has previously been observed in male Ae. vexans in the Lower Columbia River in the western United States, where males were also observed to survive up to 24 days after their habitat was marked by a aerosolized stain (Stage et al. 1937). Alternatively, the greater number of dust particles may not be due to residual dust around the release site, but simply due to the decrease in activity (which would result in less dust lost) that is implied by remaining closer to the emergence site. Additionally, it is important to note that 1) the relative species diversity (expressed as number of species observed per specimens recaptured) was much higher among mosquitoes with greater than two visible dust particles, and 2) the greater the number of days elapsed following release, the greater the diversity of species recaptured (Table 13). This supports the idea that despite measures to prevent contamination at the release site (rinsing enclosures and use of tarpaulins to contain dust), mosquitoes of many different species present at or immigrating to the release site may have marked themselves after releases had occurred.

As expected, mosquitoes with fewer visible dust particles were more likely to have flown further than those with a greater number of dust particles (Table 13). This suggests that mosquitoes lose much of their dust particles in flight after an average dispersal distance of $4.4 \mathrm{~km}$ from their site of emergence. This supports my claim that the dispersal distances 
Table 13. Summary of dust coverage on recaptured mosquitoes in 2017 . The total number of species observed is shown in parentheses next to the number of species observed per number of specimens captured.

\begin{tabular}{|c|c|c|c|c|c|}
\hline $\begin{array}{c}\text { \# of visible } \\
\text { dust particles }\end{array}$ & Individuals (\%) & $\begin{array}{l}\text { Average \# of days following } \\
\text { release before recapture }\end{array}$ & $\begin{array}{l}\text { Average distance } \\
\text { at recapture }(\mathrm{km})\end{array}$ & Males (\%) & $\begin{array}{l}\text { Species observed per } \\
\text { specimens recaptured }\end{array}$ \\
\hline$\geq 100$ & $2(1 \%)$ & 7.5 & 0.4 & 100 & $1.00(2)$ \\
\hline 26 to 99 & 0 & & & & \\
\hline 10 to 25 & $6 \quad(3 \%)$ & 4.4 & 1.4 & 50 & $0.67(2)$ \\
\hline 5 to 9 & $7 \quad(4 \%)$ & 5.3 & 1.3 & 43 & $0.43(3)$ \\
\hline 3 to 4 & $10 \quad(5 \%)$ & 6.3 & 3.1 & 40 & $0.30(3)$ \\
\hline 2 & $22 \quad(11 \%)$ & 7.1 & 2.7 & 14 & $0.32(7)$ \\
\hline 1 & $145(76 \%)$ & 8.2 & 3.4 & 28 & $0.07(10)$ \\
\hline Total & 192 & 7.8 & 3.1 & 29 & $0.06(11)$ \\
\hline
\end{tabular}


reported in this study are likely legitimate and not an artifact of contamination. However, given the trends reported above, this may not be true of the lifespans implied by the recaptured mosquito data. Nevertheless, some of these male specimens may be legitimately as old as they were inferred to be, and this may be influential for their reproductive potential.

From this issue of potential contamination by residual dust at the release site emerges an interesting methodological approach with respect to measuring dispersal distance and direction. So long as the age of the mosquito is not of interest, dispersal can be estimated by a more passive MRR procedure where a known natural larval habitat is treated with a large volume of stain or fluorescent dusts, as in Stage et al. (1937). As mosquitoes emerge from their larval habitats, they may be able to rest on the surrounding vegetation and selfmark. Use of different colours would enable a maximum age range to be estimated if desired. This may save resources with respect to rearing procedures.

\section{Influence of Wind}

Apart from the apparent dispersal events of interest described previously, I was not able to observe any clear influence of wind velocity in the days prior to recapture; the locations of recaptures relative to the release site did not correlate with wind direction or speed for any recaptures obtained 1-3 days following their release. From these results, it seems more likely that landscape features promoting shade and higher air moisture are more influential in guiding the movements of mosquitoes. In 2016, traps \#11 and 13 were both approximately $4 \mathrm{~km}$ north of the release site, within $2 \mathrm{~km}$ one another. However, trap \#11, located in a forested backyard overlooking the bank of the Red River, collected over four times as many recaptures as trap \#13, which was in a shallow ditch adjacent to a garden center greenhouse in a relatively open area. The same year, trap \#52, also near the Red River, recaptured 15 marked mosquitoes, whereas many traps an equal or shorter distance from the release site (i.e., traps \#15, 17, 18 and 19), but further from a river, recaptured none. Nevertheless, trap placement may also be important, as with trap \#52, where trap \#16 was nearer to and in the same general direction relative to the release site but received no recaptures. 


\section{Environmental drivers of dispersal}

In 2017, traps in shaded and riparian areas generally captured more marked mosquitoes than other traps in more open and presumed drier areas or areas with lower humidity levels. While most traps within a $4 \mathrm{~km}$ radius of the release site were in or near shaded or riparian areas, the two traps (\#7 and 10) that captured the fewest mosquitoes (2 and 3, respectively) were also in "islands" - areas with no connectivity to vegetated areas. It should also be noted that while the trap closest to the release site (\#1) in 2016 collected 34 marked specimens, it only collected 3 specimens in 2017. However, trap \#2 (not deployed in 2016) only $200 \mathrm{~m}$ away from \#1, but located in a forested area on private property, collected 19 specimens. This gives further support to the hypothesis that mosquitoes show active dispersal towards areas of greater shade and relative humidity.

Rivers also do not appear to be deterrents to dispersal, as many mosquitoes were recaptured on the opposite side of the Red River (an open distance of approximately 140 to $150 \mathrm{~m}$ ) and other streams like the Assiniboine River relative to the release site. Traps \#8 and 9, located approximately roughly $400 \mathrm{~m}$ apart on opposing banks of the Red River, recaptured similar numbers of marked mosquitoes. Of the seven mosquitoes recaptured further than $8 \mathrm{~km}$, all were recovered in traps either close $(<1 \mathrm{~km})$ to riverbanks or in shaded areas. However, as trap collection volumes are sensitive to microclimate and local topography, observations of individual mosquito recaptures at this distance should not be interpreted with too much certainty.

\section{Implication for mosquito control buffer zone}

The observed movements from MRR collections from both years confirm that many common species in the Winnipeg region can disperse several kilometres from the southern edge of the city limits into more urban residential areas. These findings show that mosquitoes commonly disperse at least $2 \mathrm{~km}$ from their release site, if not $5 \mathrm{~km}$ or further. Since flight distances approaching or exceeding $10 \mathrm{~km}$ were uncommonly observed in the 2017 studies, the current radius of the buffer zone (8 to $12 \mathrm{~km}$, usually less than $10 \mathrm{~km}$ ) appears to be reasonable. In agreement with the recommendations of Sarnekis (2002), and Verdonschot and Besse-Lototskaya (2014), clearing open areas or breaks in vegetated corridors would decrease dispersal from breeding areas to residential areas. While it would 
be unadvisable to fragment forested corridors surrounding the city which are important to the functioning of nearby ecosystems and agriculture, deployment of traps in these areas could benefit surveillance and control operations.

\section{Limitations}

Dispersal distances reported here should be applied conservatively with respect to control decisions. As pointed out by Trewin et al. (2019), describing movement with MDT, and $F R_{50}$ and $F R_{90}$, assumes dispersal to be a discrete, linear distance and requires removal of recaptured individuals from the population. When these methods assume that recaptures would have made no further movement, they most certainly underestimate dispersal. Additionally, true dispersal distances may be underestimated further due to relatively stressful release conditions, wherein mosquitoes were kept in crowded enclosures, and dusted and released during the day rather than at night when their active dispersal is most significant (Morris et al. 1991). Conversely, females that are kept in crowded enclosures prior to release may disperse further than those not as confined, as has been observed in Aedes (Ochlerotatus) taeniorhynchus (Wiedemann, 1821), though this behaviour was not seen in Culex mosquitoes (Nayar and Sauerman 1969; Nayar and Sauerman 1973). Meteorological variation may also affect flight ranges: since 2017 was particularly dry year, flight ranges may be larger when conditions are more humid and open areas more tolerable for adult mosquitoes.

It should also be recognized that "traditional" methods of dispersal metrics such as MDT and flight range are highly variable when recapture distributions are positively skewed and have long tails (Winskill et al. 2015), as is the case in these outcomes. Analysis of recapture data where outliers are removed, and smaller annuli are used for greater precision would assist in determining if the influence of outliers is inflating dispersal estimates. When $1.8 \mathrm{~km}$ annuli were used for analysis, MDT values for females were consistently larger than their $\mathrm{FR}_{50}$ values (mathematically the median distance travelled), indicating outsized influence of a few outliers. Those for males were more alike, which is expected given that only one outlier was identified as male. While all MDT values calculated with $1.8 \mathrm{~km}$ annuli are larger than those obtained with $0.5 \mathrm{~km}$ annuli, the latter still consistently exceed $3 \mathrm{~km}$. Additionally, the flight ranges within which 50\% and $90 \%$ 
of all insects are expected to disperse were reasonably similar across both analyses, despite being derived exclusively by extrapolation in the analysis using $0.5 \mathrm{~km}$ annuli. No consistent trends across species were found to suggest that mosquitoes cover greater ranges as they age than in the first seven days following emergence, regardless of annuli spacing. However, it is advisable to isolate and analyse data with consideration for differences in time spent dispersing, as these results demonstrate that appreciable mosquito dispersal activity is still occurring two to three weeks following their release.

It may be that traps with greater recapture numbers were situated in areas where there was a greater likelihood of trapping mosquitoes. This may be explained by kineses that governs mosquito movement, where certain stimuli may drive the mosquito to increase its speed and rate of turning. However, it is difficult to determine the exact influence of trap placement and what sort of environmental traits would provide this stimuli. As such, it is more prudent to interpret trap collections as measures of mosquito activity, rather than mosquito abundance.

Finally, I conclude with a discussion of the best practices for mosquito MRR studies in the future, in particular those seeking to measure dispersal across a relatively large landscape. Brust (1980) stated that "to trap adults beyond $12 \mathrm{~km}$, it appears that more than one million adults need to be marked and released at one time". However, these findings suggest that this may be unnecessary, as recapture numbers roughly equal or greater than those of Brust were obtained with 50,000 or fewer mosquitoes released. While more mosquitoes may be preferable if large samples of recaptured mosquitoes are desired, this is not necessary because strategic trap placement appears to be more important in measuring long-range dispersal. It is not so much the number of released mosquitoes that matters, but the amount of recapture effort.

If one were to repeat this experiment, it would be advisable to deploy more NJLTs to increase trapping effort, especially at greater distances from the release site. Additionally, if it is unfeasible to place traps to surround the release site such that they are present in all directions, it would be desirable to use more than one release site. Here, we employed only one release site and found that it was difficult to accommodate traps in the more rural parts of the trapping radius. This meant that more traps represented the northern, 
more urban portion of the trapping radius, rendering it unclear whether mosquitoes preferentially fly great distances into urban areas over rural areas.

In addition, one may want to consider the ethical ramifications of releasing more mosquitoes than necessary, even if they do not carry known pathogens. Most MRR experiments result in a net reduction of mosquitoes because of an increase in trapping efforts (Benedict et al. 2018). But in 2017, a greater number of female mosquitoes were released than were recaptured. In fact, in 75 out of $93(80 \%)$ recapture events (unique trap and day), traps collections would consist of $20 \%$ or more marked mosquitoes. In 31 of these instances, $100 \%$ of the sample consisted of recaptures (mostly single individuals, though several examples where the sample consisted of 2-5 specimens were observed). It is not understood if or how lower mosquito population density could affect the dispersal behaviors measured here. Future MRR experiments should avoid taking place during very dry conditions.

Webb and Russell (2019) noted that an operator failure to identify some marked and recaptured mosquitoes could result in underestimated dispersal distances. This may be true of our study: marked mosquitoes were found to have more fluorescent dust visible when viewed under a microscope in dark field with fluorescence illumination (used to photograph marked specimens, see Figure 23) than when viewed with the compound microscope set-up used to identify them initially. This implies that some marked mosquitoes whose dust markings were undetectable during initial processing, whether due to initial coverage or loss because of dispersal, may have been missed. We echo their recommendation that recaptured specimens should be closely inspected with the best resources available. This can be very challenging when many thousands of adults must be examined in such studies and as recapture rates are generally low it is necessary to examine as many individuals as possible to establish movement patterns.

It would also be best practice to retain some marked adults from at least one enclosure per release. One could use these specimens to assess the extent of dust coverage from the method used here. These could also be used to determine the extent of within-trap dust transfer (and maybe loss) onto bycatch, giving false negatives, or onto unmarked mosquitoes, giving false positives if wild mosquitoes are marked. A separate study 
focusing on the questions of dust retention and transfer would clarify the effectiveness of the marking procedures used.

A collection of marked adults could also inform the species composition of released adults as a representative sample of the release cohorts and corroborate the recapture results. Differences in the species composition of the recaptures between years, particularly the appearance of Cs. inornata in the 2017 recaptures, can be attributed to the origin of the field-caught larvae (not taken from a floodplain as in 2016). However, the plausibility that all marked specimens in 2017 were reared from the simulated environment in the rearing pools is dubious, as some species are purported to require specialized environments to either oviposit or survive to adulthood. Additionally, the number of apparently long-lived males, as well as the trend of relative species diversity increasing with greater dust coverage at recapture supports the hypothesis that mosquitoes may be self-marking at the release site after marking procedures have ended. We recommend future MRR studies prioritize the collection of larval samples from rearing pools, ideally rearing them to adulthood for more accurate identification. This would provide evidence for the origin of the recaptured mosquitoes.

As I conclude this chapter, I will address the presence of two species among the recaptured specimens that may be unusual. Coquillettidia perturbans was recaptured during both 2016 (7 specimens) and 2017 (11 specimens) and are typically associated with the cattails or rushes (Typha latifolia L. and Juncus L. spp.) to which they attach their larval siphons in order to breathe (Sérandour et al. 2010; Poirier and Berry 2011). To our knowledge, neither of these plants were present in the rearing enclosures. However, there are many ditches within several kilometers that host high densities these species. It may be that $C q$. perturbans, as well as other species such as Ae. vexans, that do not readily oviposit in artificial containers, were incidentally marked while resting in the nearby grass either during or after the mark and release procedures. In 2017, details about the location and level of dust coverage on each recaptured mosquito were recorded. The majority $(75 \%)$ presented with only one visible particle of dust, most commonly (30\%) seen on one of the wings. While incidental marking of other species present at the release site may be occurring, if this were the case, we would expect that these would show a consistent 
marking pattern. However, no specific trends in the locations of dust markings between recaptured specimens could be identified.

It is also possible that under certain conditions they can use less-than-ideal environments for oviposition and larval development. Similarly, Ae. vexans are known to lay their eggs singly above the waterline on damp soils and require flooding to hatch (O'Malley 1990). While the Ae. vexans were collected from a flooded area in 2016, their prominent appearance in the marked recaptures in 2017 suggests they may be able to oviposit on standing water or already-waterlogged soils when conditions are dry, as has been speculated in Headlee (1945). This flexibility would lend credence to their persistence in the Winnipeg region and elsewhere. Alternatively, as was suggested for Cq. perturbans, the presence of Ae. vexans in traps may reflect specimens incidentally marked while resting in the nearby grass either during or after the mark and release procedures. 


\section{ChAPTER 4 - SPATIAl Distribution MODElling}

Title: Modelling of adult mosquito distribution in the Winnipeg region using Random Forest regression

\section{0 - Abstract}

Background: Successful suppression of mosquito populations is often challenging in urban areas due to the ubiquity of cryptic habitats and high spatial heterogeneity. Generalized insecticidal treatment regimes may be a non-economical use of resources by mosquito control practitioners if they do not prioritize areas where mosquito population are likely to be densest. Distribution models developed using data from mosquito surveillance traps and landscape-level variables can provide insight into mosquito movement and areas of refuge. The models developed in this study are intended to characterize mosquito distribution in the Winnipeg, Manitoba region in central Canada for the purpose of optimizing municipal mosquito control measures.

Methods: Nine consecutive years of data from 28 New Jersey light traps were used to model mosquito distribution in unsampled areas. Explanatory variables related to the physical landscape were obtained from Sentinel-2 satellite imagery. These were used to characterize trap environments and to subsequently explain spatial variation on a speciesby-species basis using Random Forest (RF) regression models.

Results: Random Forest models explained 31 to $57 \%$ of the variance observed between traps. Risk maps produced by the models showed high concentrations of Aedes vexans and Culex restuans clustered within riparian areas closest to rivers. Conversely, population hotspots for Culex tarsalis were located near or beyond city limits.

Conclusions: Predicted distributions for Ae. vexans and $C x$. tarsalis populations reflect their reproductive behaviours and require separate strategies for abatement. Implications of these findings with respect to the potential efficacy of maintained mosquito surveillance efforts within a $10 \mathrm{~km}$ buffer size surrounding urban city limits are also discussed.

Keywords: species distribution modelling, mosquito nuisance, buffer zone, Culicidae, Aedes vexans, Culex tarsalis, Random Forest 


\section{1 - Introduction}

The nuisance and threat of pathogen transmission posed by vectors such as mosquitoes has long been recognized as a spatial problem (Ostfeld et al. 2005). While swamps and other damp wilderness areas come to mind as typical mosquito habitats, their populations persist wherever they find the physical or biological conditions to survive. This is especially true of cities, where urban infrastructure, residences, and vegetation form a complex mosaic of cryptic but suitable habitats. Most mosquito abatement programs follow principles of Integrated Pest Management (IPM) and work pre-emptively to prevent the development of elevated mosquito populations. However, the heterogeneity of urban environments often limits the number of larval habitats that can be removed or treated. When flooded with rain, catch basins, uneven pavement, and personal household effects (like flowerpots and rain barrels) create inconspicuous and predator-less habitats for mosquito larvae.

While it is more advisable to focus abatement on mosquitoes in their larval stage than as adults, this is often difficult to achieve in urban areas given the ubiquity of cryptic larval habitats. Consequently, many municipalities employ mass application of insecticides by way of ultra-low volume (ULV) aerosol sprays that kill adult mosquitoes in flight upon contact (US Environmental Protection Agency 2017). However, these generalized treatment regimes are often controversial among citizens because of potential human and environmental health risks (Roche 2002). In the interests of municipal IMM practitioners and residential stakeholders alike, the use of adulticides (by ULV applications or other means) should be optimized such that areas with the greatest risk of elevated mosquito populations are prioritized for treatment.

Historically, counts from mosquito traps have been a standard measure of mosquito activity used in IMM surveillance programs. Increasingly, practitioners are coupling this information with spatial data to improve the specificity of their insecticide applications. The emergence of remote sensing and geographic information systems technologies (GIS) have enabled their efforts to disentangle the influence of environmental features on mosquito populations. To this end, many have modeled the spatial relationships between mosquitoes and their surroundings to better understand their ecology and behaviour. 
Models for mosquito distribution are often projected onto maps of the study area, which can allow users to visualize the "risk" of encountering abundant mosquito populations in unsampled areas (Kitron 2000; Chuang, Henebry, et al. 2012; Ong et al. 2018). The resulting risk maps are similar to species distribution maps produced in conservation studies (Mi et al. 2017; Westwood et al. 2019). They may show the precise location of potential mosquito "hotspots", or areas of greater mosquito density, which can then be targeted to improve the efficacy and efficiency of mosquito control measures.

This study was focused on the development of mosquito distribution models for four prevalent mosquito species in the Winnipeg region in central Canada: Aedes vexans (Meigen, 1830), Culex tarsalis Coquillett, 1896, Culex restuans Theobald, 1901, and Aedes (Ochlerotatus) dorsalis (Meigen, 1830). In the province of Manitoba, where Winnipeg is located, the mosquito has been jokingly referred to as the "provincial bird". As part of their IMM strategy to suppress mosquito populations, the city's municipal insect control branch (ICB) extends their surveillance and treatment operations beyond the city limits by 8 to 12 $\mathrm{km}$. However, scientific support for the effectiveness of control measures in the buffer zone around the urban area is limited. Subsequently, it is relevant to ask if the resources used to maintain buffer zone control activities are cost effective. In other words, do mosquitoes outside of city limits contribute significantly to nuisance or transmission risk within the urban area? While the applicability of this knowledge in Winnipeg is mainly in the abatement of nuisance mosquitoes, it may also be important in preventing the future establishment of invasive mosquito species and any potential pathogens they may spread.

Among studies of mosquito habitats, there are often consistent patterns observed in the spatial distribution of mosquito species such as Ae. vexans and Cx. tarsalis. Most studies have shown that Ae. vexans prefer to use understory vegetation (Bidlingmayer 1971; Mullen 1971; Burkett-Cadena et al. 2013) and wetlands (Moncayo et al. 2000; Trawinski and Mackay 2010; Giordano et al. 2018) over other habitat types. Populations of $C x$. tarsalis have been often found to have an affinity for drier areas, such as grassy or cultivated land (Diuk-Wasser et al. 2006; Nielsen et al. 2008; Chuang et al. 2011; Yoo et al. 2016). It is tempting to extrapolate the results of these studies in other regions with the same species, but this is inadvisable given the variation in spatial or temporal distribution 
across studies. For example, findings that suggest opposite habitat preferences for both mosquitoes species previously mentioned are also present in the literature (Diuk-Wasser et al. 2006; Giordano et al. 2018). As mosquito population distributions vary depending on the specific geographic characteristics of each locality, as well as the period of study, models must be tailored to the areas where the data used to develop them were collected (Sallam, Fizer, et al. 2017).

When developing models to predict future mosquito distribution, climatic or meteorological data are often used as explanatory variables (Kolivras 2006; Chuang et al. 2011). While these factors do indeed contribute to the distribution of mosquito populations (Kovats et al. 2001), the spatial extent represented by each measurement is often too large for use in high-resolution models, which are necessary for characterization of urban areas. Given this limitation, the objective of this study was to model mosquito distribution in the Winnipeg region using only landscape-based variables. These included land use and/or land cover (LULC) classes and other metrics such as vegetation and wetness indices that were used to describe heterogeneity within a geographic extent. I hypothesized that mosquito distributions would reflect species-specific reproduction and feeding adaptations.

The goal in developing models was to determine which variables are most influential in providing mosquito habitat for each species in question. This information was then used to derive risk maps, which could be used to specify locations are most likely to harbour elevated mosquito populations and contribute to nuisance and potential pathogen transmission. As the study extent also encompassed the rural area around Winnipeg, the models were also used to predict mosquito distribution in areas outside of the city. This enabled an assessment of the potential efficacy of the buffer control zone around the city in suppressing mosquito populations. Lastly, given the wide variety of methodological approaches used to model mosquito distribution, I hope to present the following methods as a standard procedure based upon my review of the literature on this topic (See Ch. 2). 


\section{2 - Methods}

\subsection{1 - Study site}

Data from the Winnipeg ICB archival mosquito surveillance records were obtained for City of Winnipeg and the surrounding region in Manitoba, Canada. See 3.2.1 for a description of this study area.

\subsection{2. - Mosquito data}

A summary of the data extraction procedure is described in Figure 24. Archival mosquito surveillance data were obtained from the City of Winnipeg ICB, which had digitized records of New Jersey Light Trap (NJLT) collections from 1991 to 2017. Across these 27 years, 61 individual trap locations were used although many did not operate concurrently, or for more than a few consecutive years. Traps with fewer than 4 consecutive years of monitoring were eliminated from the dataset, leaving 37 traps, though these did not all operate concurrently between 1991 and 2017. The dataset was then reduced to optimize for the greatest number of traps which were concurrently operating for the longest consecutive-year period. The final mosquito activity dataset encompassed the collections from 28 NJLTs that operated over a 9-year period from 2007 to 2015 (Figure 25). This dataset also included the numbers of female mosquitoes for several dominant species of interest for each collection event. From 2007 to 2014, total numbers of female Ae. vexans, Cx. tarsalis, Cx. restuans and Ae. dorsalis were recorded. In 2015, Ae. dorsalis were no longer being identified in each collection, having been replaced by Coquillettidia perturbans (Walker, 1856) as a species of interest. Once per week, ICB staff would identify all female mosquitoes to species, but species other than those listed above almost always comprised less than $1 \%$ of all specimens combined. 


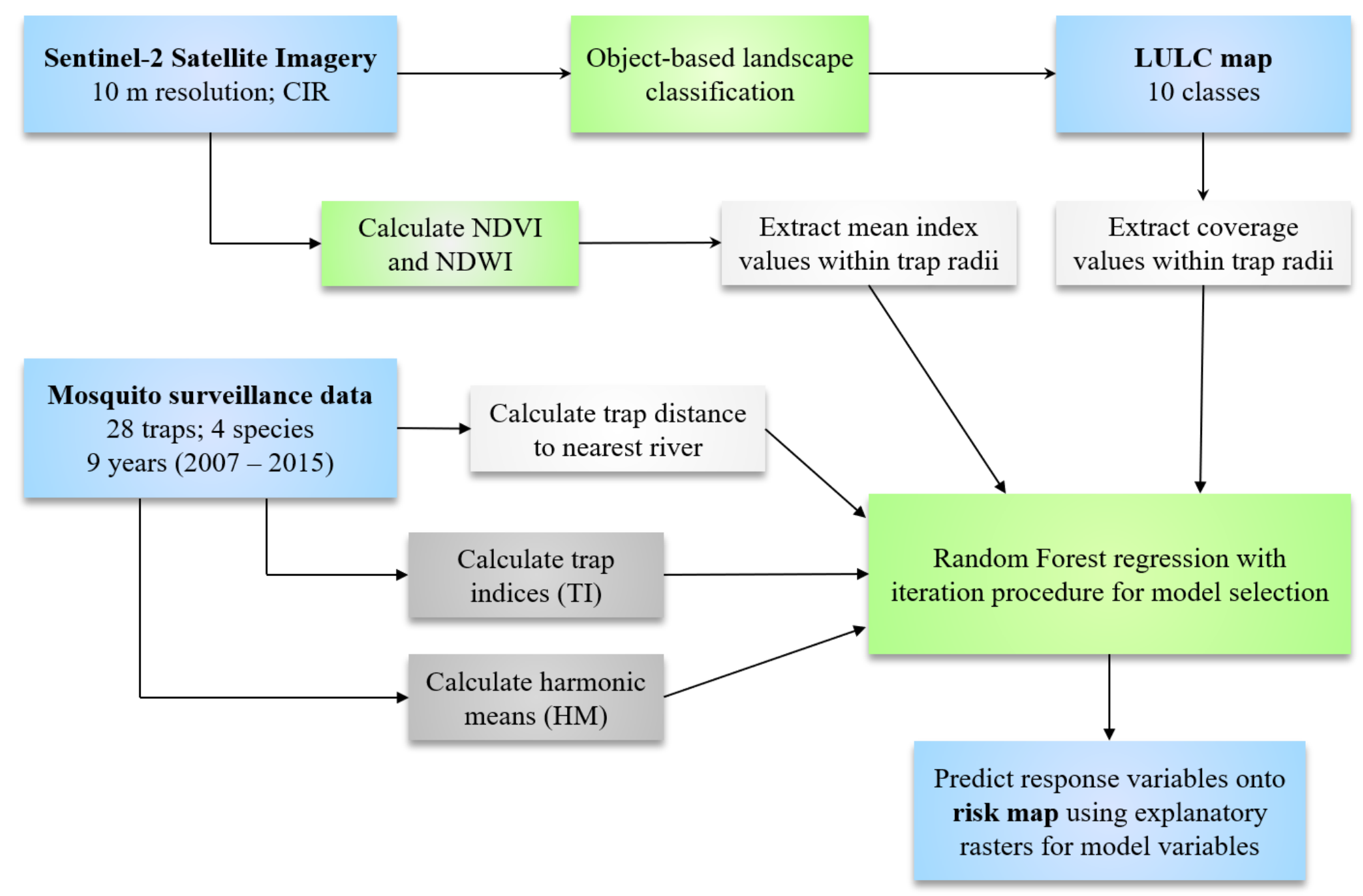

Figure 24. Workflow summary for generating species-specific mosquito risk maps in the Winnipeg, MB region. Blue items indicate data sources or products. Data processing steps are shown in green. Explanatory variables are shown in light grey and dark grey items indicate response variables. 


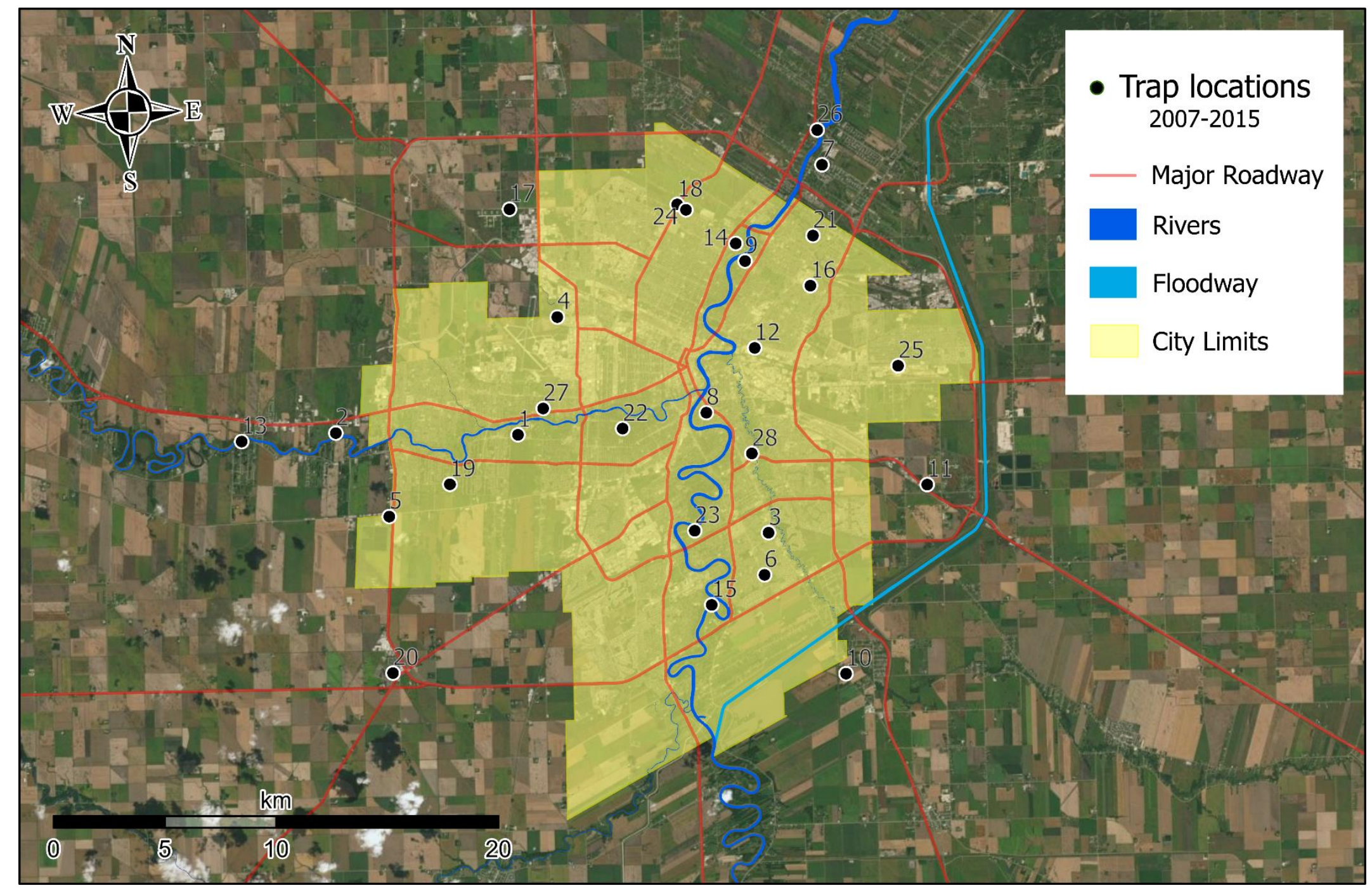

Figure 25. Locations of the 28 New Jersey light traps (NJLTs) operating concurrently from 2007 to 2015. 
Within the final dataset, the frequency of collection varied according to the mosquito volume observed throughout individual seasons, as well as year to year. During certain years, collections were processed 7 days a week, whereas other years never exceeded 5 days per week. During the early and late periods of the season (i.e., May, September, October), it is not uncommon for collections to be processed only every 2 to 4 days (up to a maximum of 6 days). As such, the mosquito numbers recorded for each collection event were standardized to reflect numbers collected on a per-day basis. This was done by dividing the number of mosquitoes collected during each collection event by the number of trap nights elapsed since the previous collection event. Standardized collection events for each trap were then replicated according to the number of trap nights elapsed since the trap was last emptied. This was to ensure that collection events contributed proportionally to the number of trap nights they were active. To better capture general trends in activity throughout a typical mosquito season, collection events were excluded from the final dataset if they were recorded earlier than May $1^{\text {st }}$ or later than September $31^{\text {st }}$.

Multi-year surveillance datasets (>5 years) from mosquito traps are rare in spatial modelling studies. When multi-year data are utilized usually some form of standardizing approach is often used when comparing mosquito counts across traps with differential trapping efforts (DeMets et al. 2020; Giordano et al. 2020). Arithmetic means are also sometimes employed as representative values for mosquito activity at a given locale (Chuang et al. 2011; Chuang, Henebry, et al. 2012). However, given that mosquito numbers may vary from year to year by multiple orders of magnitude (dependent on climatic conditions), calculating the arithmetic average of trap numbers from all years can lead to an inaccurate method of obtaining a representative value of a locality's mosquito activity (McNichol 2018). The method of calculating a representative value for mosquito activity must be robust to outliers to reduce the bias from years where seasonal variation allowed for generally sparser or denser populations overall. Therefore, I devised a standardizing procedure to calculate two different values; a trap index (TI) and a harmonic mean (HM), which are meant to represent mosquito activity at each trap location irrespective of yearly variation. Both TI and HM were calculated and used in separate analyses for each of $A e$. 
vexans $(N=609,692), C x$. tarsalis $(N=14,608), C x$. restuans $(\mathrm{N}=10,100)$, and Ae. dorsalis $(\mathrm{N}=3,743)$.

Trap indices (TIs) were derived from individual trap collection events $\left(x_{i, j}\right)$ weighted using the reciprocal of the total number of mosquitoes collected $(w)$ during the year $(j)$ of the collection event $(i)$ for a given trap $(x)$. This allowed for standardization of counts from collection events such that they expressed proportions of the total number of mosquitoes trapped each year, rather than absolute numbers. To obtain TI, these standardized values were summed for an individual trap over all years and then multiplied by the mean yearly total for all traps $\left(\bar{x}_{w}\right)$, as in the following formula:

$$
\mathrm{TI}=\bar{x}_{w} \sum_{j=1}^{n} x_{i, j} \frac{1}{w_{j}}, \quad \text { where } \quad w_{j}=\sum_{i=1}^{n} x_{i, j}
$$

The harmonic mean (HM) of each trap's total yearly counts was also generated as another representative measure of trap numbers. Harmonic means are often employed when finding the average of a set of rates or ratios where both the numerator and denominators are variable. As such, the HM accommodates two dimensions of multiplicative relationships (Manikandan 2011) and is more suited to generalizing the datasets with lognormal (left-skewed) distributions and large outliers that are common in ecological datasets (Karlin 1968; Dennis and Patil 1988). Although seldom applied to ecological data, the HM is sometimes used when calculating the effective population (often expressed as $N_{e}$ ), where environmental effects on successive generations are assumed to be multiplicative (Karlin 1968). Here, the harmonic mean represents an average of total yearly counts for a single trap across multiple surveillance seasons (analogous to successive generations in $N_{e}$ ). The harmonic mean was calculated as the reciprocal of the arithmetic mean of the reciprocals of the total yearly counts, and is expressed as follows (adapted from Zar 2010):

$$
\mathrm{HM}=\frac{M}{\sum_{j=1}^{m} \frac{1}{\sum_{i=1}^{n} x_{i, j}}}
$$


Where $x_{i, j}$ is the $i^{\text {th }}$ collection event in the $j^{\text {th }}$ year and $M$ is the number of years considered (eight for Ae. dorsalis, nine for all other species). Note that while the HM is relatively insensitive to large outliers, it is not equally so to small outliers, and tends to skew left relative to the arithmetic mean. In contrast to the TIs, which are dependent on the relative mosquito activity of the other traps (within the same year), the HMs of different traps are independent of one another. Both values were used to produce species distribution models, as differences between them may highlight different environmental predictors or long-term trends. Both the TI and HM values generated from the trap data were tested for spatial autocorrelation using Global Moran's I tool. The average distance between traps was calculated using Average Nearest Neighbor. Both operations were performed in ArcGIS Pro 2.5.1 (ESRI Inc., Redlands, CA).

\subsection{3. - Spatial data}

No thematic maps of LULC data for the Winnipeg area were available at a resolution appropriate for a within-city spatial analysis $(\leq 10 \mathrm{~m}$ per pixel, as recommended by Anderson et al. (1976) and Herold et al. (2003). While orthographic photography tiles of the city at very high resolution $(7.5 \mathrm{~cm})$ were available (City of Winnipeg 2018), these only captured pixel data for the red, green and blue (RGB) bands of light. Use of these tiles would have precluded the inclusion of often important indices as potential predictors of mosquito activity, such as the Normalized Difference Vegetation Index (NDVI) (Brown et al. 2008; Chuang, Henebry, et al. 2012; Demets et al. 2020) and the Normalized Difference Wetness Index (McFeeters 2013; Wiese et al. 2019). These index values require measurements in the near-infrared (NIR) range, and so cannot be obtained from the RGB orthophotos. Additionally, at least 5 traps fell beyond the spatial extent of these orthophotos. If they were to be used, these trap locations would need to be excluded, or characterized by some other means at a coarser scale than those traps within the photos. If mosquito dispersal from beyond the city limits poses a potential problem for nuisance mosquito populations, information from traps outside the city perimeter would be necessary to identify this problem.

To obtain data on the LULC characteristics of each trap's surroundings, I did an object-based landscape classification of multispectral satellite imagery. The use of 
multispectral $10 \mathrm{~m}$ resolution imagery from the remote sensing satellite programme Sentinel-2 (obtained from the Copernicus Open Access Hub) was a compromise between temporal, spatial, and spectral resolution. The Sentinel-2 sensors can measure emitted or reflected energy in the NIR range, as well as in the RGB bands. However, since the first Sentinel was launched in June 2015, no cloud-free imagery products of the Winnipeg area were collected in the summer of 2015. Instead, cloud-free imagery from June 30, 2019 was selected with the assumption that the land cover and use throughout the study area remained relatively consistent in Winnipeg from 2007 to 2015 (Statistics Canada 2016). Mosquito numbers reach their peak in the Winnipeg area in either June or July. As a paired t-test found no significant differences between the numbers of female mosquitoes collected in June and July from 2007 to 2015, imagery from late June was deemed an appropriate midpoint.

The object-based landscape classification workflow and extraction of all spatial data were completed in ArcGIS Pro 2.5.1. The Sentinel-2 imagery was clipped to the study area and a false colour composite image was created using the NIR, green, and blue wavelength bands. Together, these highlighted variations in primary productivity (vegetation) across the landscape. Using the Segment Mean Shift tool (Spatial Analyst), I created a segmented image (Figure 26) to decrease the data size and complexity of the imagery prior to classification. Segmentation is a process that divides the imagery into groups of multiple adjacent pixels that represent homogenous regions relative to their surroundings (Pal and Pal 1993). The Segment Mean Shift tool uses a region-based segmentation algorithm, meaning that it recognizes objects starting from the inner pixels rather than those at its edges, as in edge-based segmentation methods (Hossain and Chen 2019). Parameter selection for this operation specified the maximum spatial and spectral detail (20) and a minimum segment size of 5 pixels to preserve as much detail as possible.

A 446-sample training dataset was produced using 500 randomly generated points within a $4 \mathrm{~km}$ radius of the trapping locations. At each point, a sample "object" was delineated as an individual polygon feature. If a point fell on the boundary of two or more objects, all objects were delineated. Points that fell within already sampled objects were skipped as to not be sampled twice. Each object corresponded with one of ten LULC 
classes, where seven were mutually exclusive and exhaustive land cover classes. Four of these land cover types were defined by the coverage and type of vegetation present: cultivated land, bare ground (or sparsely vegetated), grass (or open area), and forest (or densely vegetated canopy). Pavement, standing water, and river water were also included among these land cover classes. The remaining three classes were zoning designations residential, commercial (or public), and industrial - and characterized land use in contiguous built-up areas, such as residential neighborhoods or city blocks. These land use classes were mutually exclusive from one another but could within them include smaller objects made of non-water land cover classes listed above. All LULC classes are described in Table 14. The rationale of including zoning designations was that the relative amount and type of vegetation differs between them. Vegetation in residential neighborhoods is predominantly lawns and trees, whereas commercial and industrial areas have less vegetation overall.

The classification of the study area into a thematic map composed of the above LULC classes was performed by a Support Vector Machine (SVM) algorithm (henceforth "the classifier"). The classifier was trained on the samples from the segmented image using all segment attribute options except rectangularity. The SVM is a supervised machine learning algorithm that is used in classification and non-linear regression analyses (Cortes and Vapnik 1995). It is a non-parametric approach that does not require the dataset to adhere any particular distribution for best statistical practice (Everingham et al. 2007). Support Vector Machine algorithms have been shown to perform well in landscape classification studies (Khatami et al. 2016; Thanh Noi and Kappas 2017), as they are less sensitive to noise and more robust to unbalanced datasets relative to more common algorithms (Adam et al. 2014). In their studies of evaluating the performance of machine learning classifiers on remotely sensed data, Everingham et al. (2007) and Adam et al. (2014) discuss basic theory and include further reading pertaining to SVM.

Within the thematic map generated by the classifier (Figure 26), large or conspicuous areas that had been classified incorrectly were manually reclassified for greater accuracy. The accuracy of this final image was assessed by randomly generating 
Table 14. Description of all LULC classes used in the object-based landscape classification.

\begin{tabular}{|c|c|}
\hline Variable Name & Description \\
\hline RESIDENTIAL & Built land generally used for residential purposes where housing predominates. \\
\hline COMMERCIAL & $\begin{array}{l}\text { Built land generally used for commercial purposes, primarily providing space to } \\
\text { retail and service businesses. Land with public or administrative buildings such } \\
\text { as community halls or government offices have also been included. }\end{array}$ \\
\hline INDUSTRIAL & $\begin{array}{l}\text { Built land generally used for industrial purposes. Plants, factories, storage } \\
\text { facilities and airports are examples of common buildings in industrial areas. }\end{array}$ \\
\hline PAVEMENT & Surface that is paved with asphalt or content, such as a road or parking lot. \\
\hline CULTIVATED & $\begin{array}{l}\text { Arable land that is worked by raising crop monocultures. Common crop species } \\
\text { in the Winnipeg region include wheat, canola, and flaxseed. Land in fallow is } \\
\text { included within this definition. }\end{array}$ \\
\hline FOREST & Densely vegetated area characterized by tree canopy. \\
\hline GRASS & $\begin{array}{l}\text { Open area, such as a field or lawn that is generally free of trees and dominated } \\
\text { by low grasses and forbs. }\end{array}$ \\
\hline GROUND & Bare ground where soil is exposed, and vegetation is absent or sparse. \\
\hline RIVER & Flowing water course greater than 10 metres in width. \\
\hline WATER & Standing water feature such as a pond, reservoir, or pool. \\
\hline
\end{tabular}

500 points, where 50 points where generated for each class, as recommended by Congalton (1991). Each point was reviewed individually to determine if the classifier had correctly identified its location on the thematic map with the "true" LULC class. Expert knowledge augmented by Landsat Imagery from 2014 and 2015 provided by Google Earth Pro were used as a reference to determine the accuracy of the classification.

A confusion (or error) matrix (Table 15) was generated using data from the accuracy assessment to calculate the producer's accuracy, user's accuracy, and Cohen's Kappa (к) coefficient (Cohen 1960; Congalton 1991). The producer's accuracy is a measure of how likely a random pixel from the reference data will be correctly classified by the classifier. It is obtained by dividing the number of accurately classified reference 


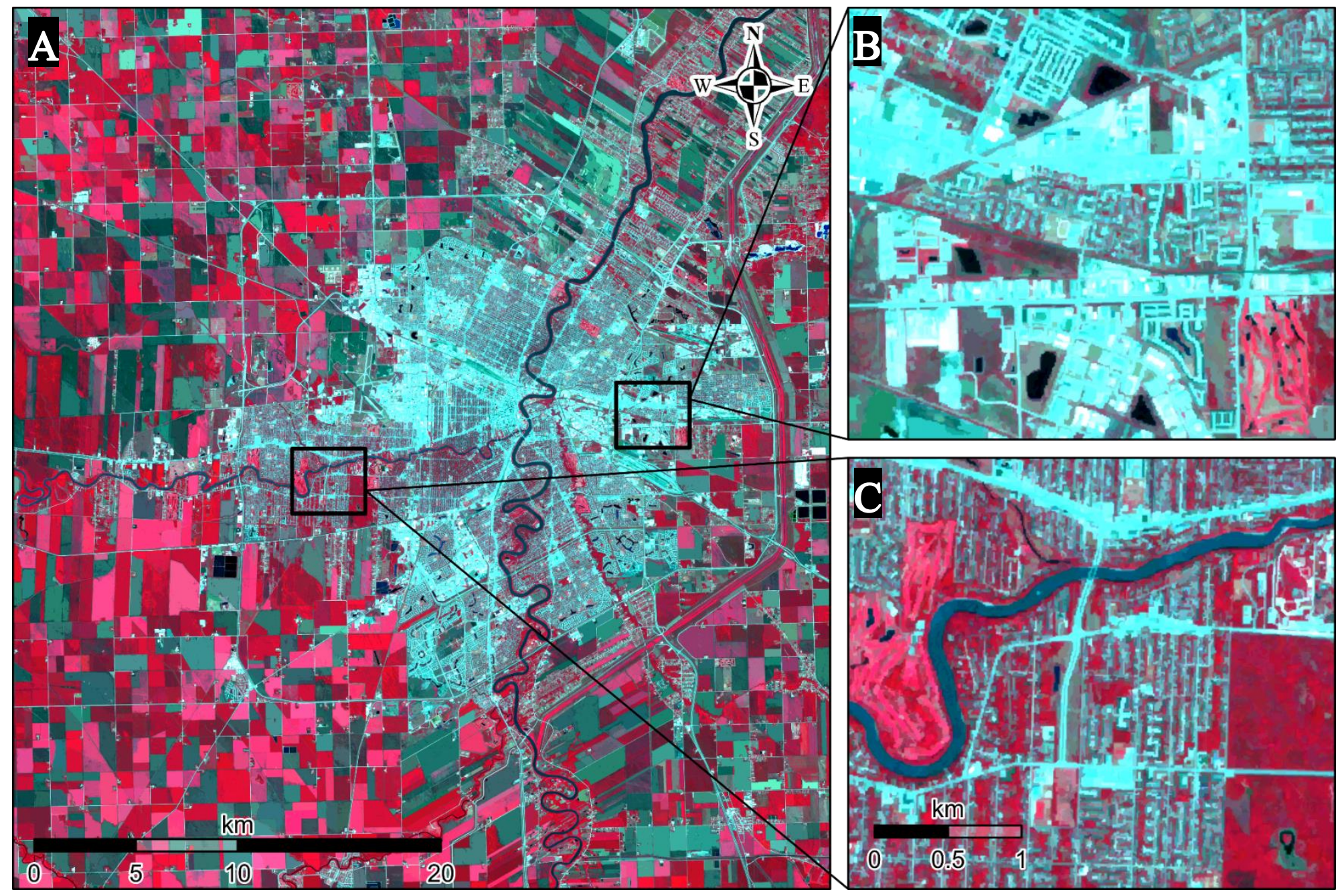

Figure 26. A. Segmented $10 \mathrm{~m}$ resolution image generated from a false colour composite image of the study area created using the NIR, green, and blue wavelength bands. Insets B and C show examples of different regions seen at the pixel-level. Imagery was obtained from the Copernicus Programme Sentinel-2 mission on June 30 2019. 
Table 15. Confusion Matrix results for the LULC classification performed with the nearinfrared, blue, and green bands from 2019 Sentinel-2 imagery. Reference data used for the accuracy assessment are in columns, while rows represent the classification generated from these data.

\begin{tabular}{|c|c|c|c|c|c|c|c|c|c|c|c|c|c|}
\hline & Class & 1 & 2 & 3 & 4 & 5 & 6 & 7 & 8 & 9 & 10 & Total & $\begin{array}{c}\text { User's } \\
\text { accuracy (\%) }\end{array}$ \\
\hline 1 & Cultivated land & 50 & 0 & 0 & 0 & 0 & 0 & 0 & 0 & 0 & 0 & 50 & 100. \\
\hline 2 & River & 4 & 39 & 6 & 0 & 0 & 0 & 0 & 0 & 1 & 0 & 50 & 78 \\
\hline 3 & Standing Water & 1 & 3 & 38 & 0 & 0 & 0 & 0 & 2 & 4 & 1 & 49 & 78 \\
\hline 4 & Residential & 4 & 0 & 0 & 42 & 0 & 0 & 0 & 1 & 2 & 1 & 50 & 84 \\
\hline 5 & Commercial & 0 & 0 & 0 & 12 & 27 & 2 & 8 & 0 & 1 & 0 & 50 & 54 \\
\hline 6 & Industrial & 0 & 0 & 0 & 2 & 0 & 29 & 4 & 15 & 0 & 0 & 50 & 58 \\
\hline 7 & Pavement & 2 & 0 & 0 & 0 & 0 & 0 & 46 & 2 & 0 & 0 & 50 & 92 \\
\hline 8 & Bare Ground. & 9 & 0 & 0 & 2 & 0 & 0 & 1 & 38 & 0 & 0 & 50 & 76 \\
\hline 9 & Grass & 12 & 0 & 0 & 0 & 0 & 0 & 0 & 1 & 36 & 1 & 50 & 72. \\
\hline \multirow[t]{5}{*}{10} & Forest. & 8 & 0 & 0 & 0 & 0 & 0 & 0 & 0 & 1 & 41 & 50 & 82 \\
\hline & Total & 90 & 42 & 44 & 58 & 27 & 31 & 59 & 59 & 45 & 44 & 499 & \\
\hline & $\begin{array}{l}\text { Producer's } \\
\text { Accuracy (\%) }\end{array}$ & 56 & 93 & 86 & 72 & 100 & 94 & 78 & 64 & 80 & 93 & 77 & \\
\hline & $\begin{array}{l}\text { Overall } \\
\text { accuracy }(\%)\end{array}$ & 77 & & & & & & & & & & & \\
\hline & Карра & 0.75 & & & & & & & & & & & \\
\hline
\end{tabular}

pixels within a class by the total number of reference points in this class. The user's accuracy is the number of accurately classified reference pixels within a class divided by the total number of pixels assigned to that class by the classifier. It is a measure of reliability, representing the likelihood that a pixel on the map is classified accurately with respect to the reference data (and ideally, to reality).

Kappa $(\kappa)$ is a measure of how well the classification performed in comparison to a scenario in which classes were assigned to pixels at random. It ranges from -1 to 1 . If $\kappa$ is closer to -1 , the classification performed significantly worse than random, but if $\kappa$ is closer to 1, it performed significantly better than random (Congalton and Mead 1983). A $\kappa$ value approaching 0 indicates that the classification was as effective as a random 
assignment of values. A $\kappa$ value above 0.75 is generally regarded to indicate substantial (Landis and Koch 1977) if not "almost perfect" (Muñoz and Bangdiwala 1997) agreement between the classifier and the reference data. The overall accuracy of a classification is also often reported; it is the total proportion of pixels that were accurately classified in the accuracy assessment.

The final classified image (Figure 27) was used to extract spatial information within a fixed radius of each trap location. To do this, circular zonal areas with nested radii of 1000, 500, 250, 100 and $50 \mathrm{~m}$ were established around each trap. This was done to optimize for the spatial scale at which individual environmental variables can explain mosquito distribution (Diuk-Wasser et al. 2006), while also maximizing spatial heterogeneity across trap locations. Within these zonal areas, spatial data were extracted in the following procedures.

Geocoded areas where mosquito larvae are monitored and treated when present (known larval habitats) were obtained as a shapefile from the ICB. This dataset consisted of 2,145 regions listed for treatment delivered by helicopter, and 2,035 smaller individual sites monitored and treated by ground crews. The Tabulate Area tool was used to calculate the total cover of known larval habitats, as well each individual LULC class (ten total) for each trap radius. Resulting values ranged from 0 to 1 , where higher values represented traps which had a greater proportion of known larval habitats, or of a specific LULC class type within its surroundings.

The NDVI and NDWI were calculated for each pixel of the original Sentinel-2 satellite imagery. The mean value for each index within each trap radius was calculated using the Zonal Statistics tool. A shapefile provided by Manitoba Land Initiative (Government of Manitoba 2009) was used to mask rivers such that they did not contribute to the index values. Both the NDVI and NDWI are derived using the equations below (from Singh 1989 and McFeeters 1996, respectively). They are widely used to infer information about the distribution of either vegetation or surface moisture across a terrestrial landscape.

$$
\mathrm{NDVI}=\frac{N I R-\text { Red }}{N I R+\text { Red }} \quad \mathrm{NDWI}=\frac{\text { Green }-N I R}{\text { Green }+N I R}
$$




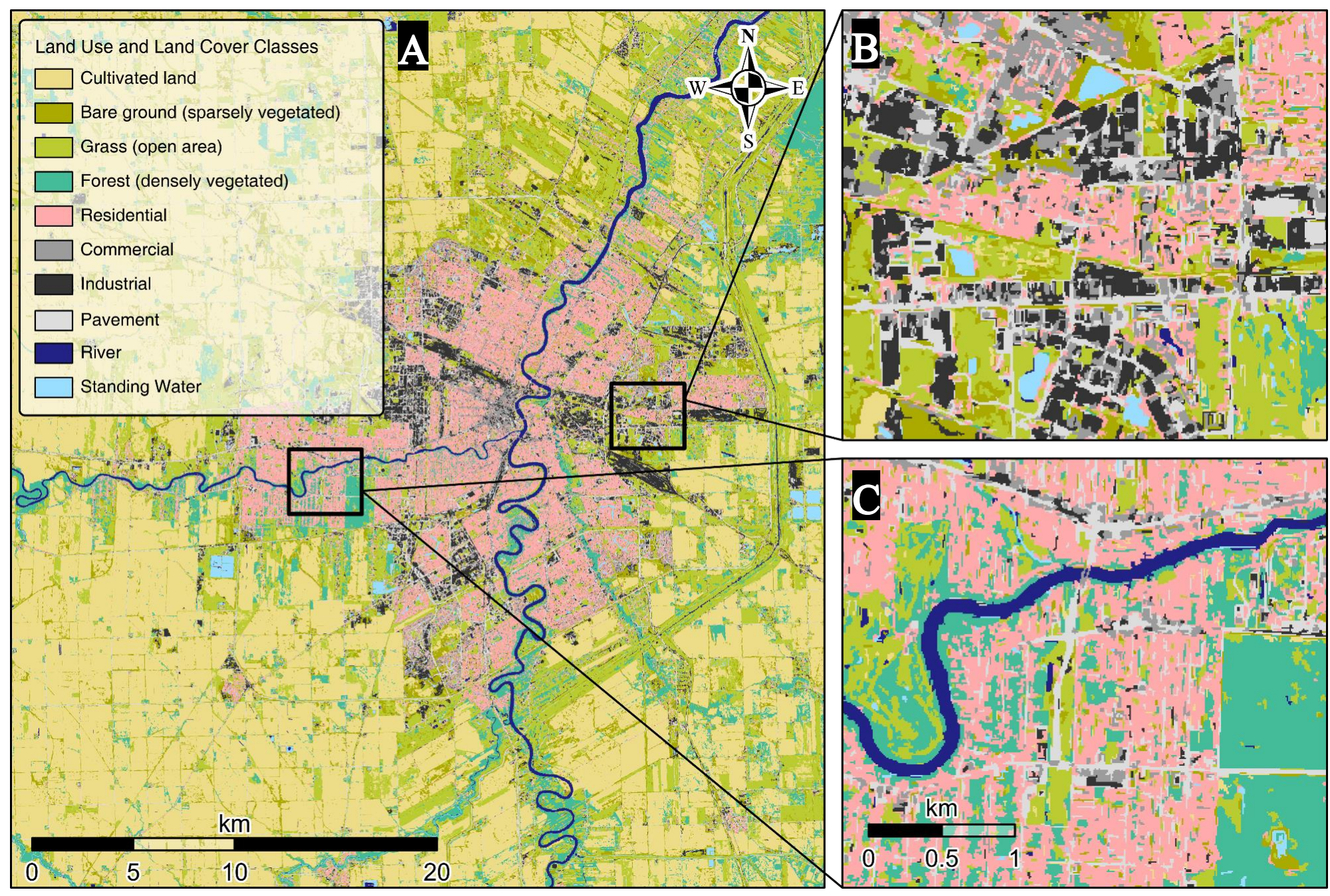

Figure 27. A. Land use and land cover map for the Winnipeg region study area, generated from an object-based landscape classification procedure using training data and a Support Vector Machine (SVM) classifier algorithm on the segmented imagery in Figure 26. Insets B and C show examples of different regions seen at the pixel-level. 
The NDVI is correlated with the abundance of productive vegetation cover (Asrar et al. 1984) and its photosynthetic capacity or health (Sellers 1985). As defined by McFeeters (1996), the NDWI has been used to delineate surface water features such as swimming pools and ponds, which are potential mosquito habitats (McFeeters 2013). Many similar studies have used NDWI as a predictor of mosquito activity (Zou et al. 2006; Brown et al. 2008; Estallo et al. 2018; Mudele et al. 2019; Piedrahita et al. 2020). However, it is important to note that all use another index by the same name proposed by Gao (1996), which measures water content in vegetation and uses the satellite measurements of reflected radiation from the short-wave infrared range (SWIR). Use of Sentinel-2 imagery at a $10 \mathrm{~m}$ resolution precluded the use of Gao's NDWI, as SWIR measurements are only measured at a $20 \mathrm{~m}$ resolution. While McFeeters' NDWI is relatively limited in its capacity to characterize plant health, its ability to identify wetland areas (Kaplan and Avdan 2017; Eid et al. 2020) may be helpful in identifying similar adult mosquito habitats.

Lastly, a spatial join was done to calculate the distance from each trap to the nearest river using the same river shapefile from the MLI above. See Appendix XVI for a summary of trap site environments.

\subsection{4 - Data analysis and mapping}

The Forest-based Classification and Regression tool in ArcGIS Pro 2.5.1 (ESRI Inc., Redlands, CA) was used to develop regression models that maximized the amount of variation in mosquito activity explained by a set of predictor variables extracted from the Sentinel-2 imagery. This tool uses the Random Forest (RF) algorithm (Breiman 2001), an ensemble machine learning method which trains a model based on a set of observations given a corresponding set of predictor variables. The model can subsequently be used to predict unknown values using the same predictors. An outline of the theory behind RF is given in Ch. 2.4. For each of Ae. vexans, Cx. tarsalis, Cx. restuans, and Ae. dorsalis, a model was developed in a stepwise manner using the natural log of the TI and HM for each trap location. The transformation was done to improve model performance as the trap counts exhibited a long-tailed distribution. While regression trees are robust to outliers and skewed distributions in predictor variables, the splitting process of RF dampens the input 
of larger values in the response variable, which have smaller variation in left skewed datasets (De'Ath and Fabricius 2000).

To find an initial model to iterate, I selected the four predictor variables most frequently listed with the greatest importance in RF models trained to generate 10,000 trees using all variables (64 in total), with the mtry parameter (number of random variables compared at each node) set to 8. Variables calculated using different zonal area sizes were not permitted to be in the same model. From the initial model, the following iterative procedure was performed on each model: 1) Each variable was removed individually, 2) Each LULC or index-based variable was adjusted individually to verify its effect on the model at all other scales (zonal area sizes), and 3) Each predictor variable not in the model was individually added at each zonal area size. The iterative model that explained a greater percentage of variance and reported a smaller MSE was then taken as the new initial model. This procedure was repeated until model performance could no longer improve. The number of trees used in each iteration was 10,000, with the mtry parameter set to either 2 (seven or fewer predictors) or 3 (more than seven predictors). The variance inflation factor (VIF) was calculated for each of the models and the iteration procedure was repeated if a model was found to have a VIF greater than five, a common threshold for indicating problematic collinearity between variables (Sheather 2009).

Final models were used to predict the risk of elevated mosquito populations on a map of the Winnipeg area using the "Predict to raster" function. To create the necessary predictor raster layers required to run this function, the Focal Statistics tool was used to create a "smoothed" raster layer for each variable in the final models (Diuk-Wasser et al. 2006). For LULC and larval habitat variables, the tool calculated the sum of the relevant pixels within the designated zonal area radius for each pixel in the raster. To obtain the zonal area proportion covered by each of these variables, these values were divided by the number of pixels contained within a circular zone of radius $r$. Since the resolution of the imagery used was $10 \mathrm{~m}, r$ was equal to the zonal area size multiplied by a factor of 1/10. Lastly, for use with models with distance to river included as a predictor, the Euclidean Distance tool was used to calculate the distance to the closest river for each of the pixels in the raster. 
In the resulting risk map rasters, I applied a back-transformation of $10^{x}$ to the value of each pixel $(x)$, which corresponded to the predicted values for the log-transformed TI or $\log$-HM at its location. This was to avoid the bias generated by log-transformed frequency distributions (Wilson et al. 1990). The maps generated by the prediction function show the probability (risk) that a unit area, indicated by a single pixel value, will have elevated mosquito activity relative to other pixels. Lastly, for each model, the variables were tested for correlation against the species activity metric being predicted using Kendall's tau coefficient $\left(\mathrm{r}_{\tau}\right)$.

\section{3 - Results}

\subsection{1 - Landscape classification accuracy}

The confusion matrix generated from the object-based landscape classification returned an overall accuracy of $77.3 \%$, with a kappa coefficient of 0.75 (Table 15). Cultivated land was the class in which other classes were most frequently misclassified, with a producer's accuracy of $56 \%$, i.e., only $56 \%$ of the reference points located on cultivated land were identified as such by the classifier. However, the user's accuracy $(100 \%)$ indicated that overall, the classifier is reliable at identifying cultivated land, i.e., all points that were located on pixels labelled as cultivated land by the classifier were also located on cultivated land in reality. Low user accuracies were obtained for commercial/public areas (54\%) as well as industrial areas (58\%), for which there were conversely high producer accuracies (100 and 93.6\%, respectively).

\subsection{2 - Spatial autocorrelation}

Across all species, Moran's I tests revealed no spatial autocorrelation ( $\mathrm{p}>0.05$ ) between traps, regardless of the activity metric used (TI or HM). The observed mean distance between traps was $2511 \mathrm{~m}$, meaning that on average, traps were slightly more dispersed than if they were to be distributed randomly.

\subsection{3 - Mapping risk with Random Forest regression models}

The model built for Ae. vexans activity using TIs performed the best out of all 8 models with $57 \%$ of the variance explained by 4 variables (Table 16). While the HM-based 
model for Ae. vexans explained less variance, both the TI and HM-based models identified forest cover at a scale of $500 \mathrm{~m}$ to be the most important variable. Both also included grass cover and residential area at $500 \mathrm{~m}$ and above as weaker predictors. Risk maps generated from Ae. vexans models show a greater concentration of elevated population risk in areas surrounding riparian areas (Figure 28 and 29). However, the map generated by the HMbased model shows more specificity with these hotspots due to the greater number of explanatory variables. Two of these variables, commercial area, and pavement (both within $100 \mathrm{~m}$ ), were both negatively correlated with the HM values for Ae. vexans.

Models for $C x$. tarsalis distribution also performed best when using TIs (53\% variance explained) to measure mosquito activity instead of HMs (31\%) (Table 16). These models included the most explanatory variables out of all the models, at 8 and 7 variables for the TI and HM-based models, respectively. Both models listed grass, ground, river and industrial LULC classes as among their predictors, but the models disagreed upon the effect scale and/or importance of these variables. Areas surrounding the city limits were identified as $C x$. tarsalis hotspots (Figure 30 and 31), with a more conservative distribution of these areas within the HM-based risk map. Both models display the inner-city area as relatively low risk. However, the HM-based model displays it as homogenously low on the risk scale. In comparison, the TI-based model has more variation in mosquito activity predictions within the inner-city.

The $C x$. restuans models behaved similarly to the Ae. vexans models, with greater concentrations of mosquitoes clustered around riparian areas (Figure 32 and 33). Patchier hotspot distribution was seen in the HM-based models. Both models included forest cover (within $500 \mathrm{~m}$ ) and rivers (within $250 \mathrm{~m}$ ) as explanatory variables (Table 16). The HMbased model (49\%) performed better than the TI-based model (37\%) in explaining the variance in $C x$. restuans activity and included a greater number of predictors.

Lastly, variance in Ae. dorsalis activity was explained slightly better by the HMbased model (38\%) than its TI counterpart (32\%) (Table 16). Both models included cultivated land within 500 to $1000 \mathrm{~m}$ as important variables. However, maps for these models disagreed on whether cultivated areas outside the city posed an average (Figure 34) 


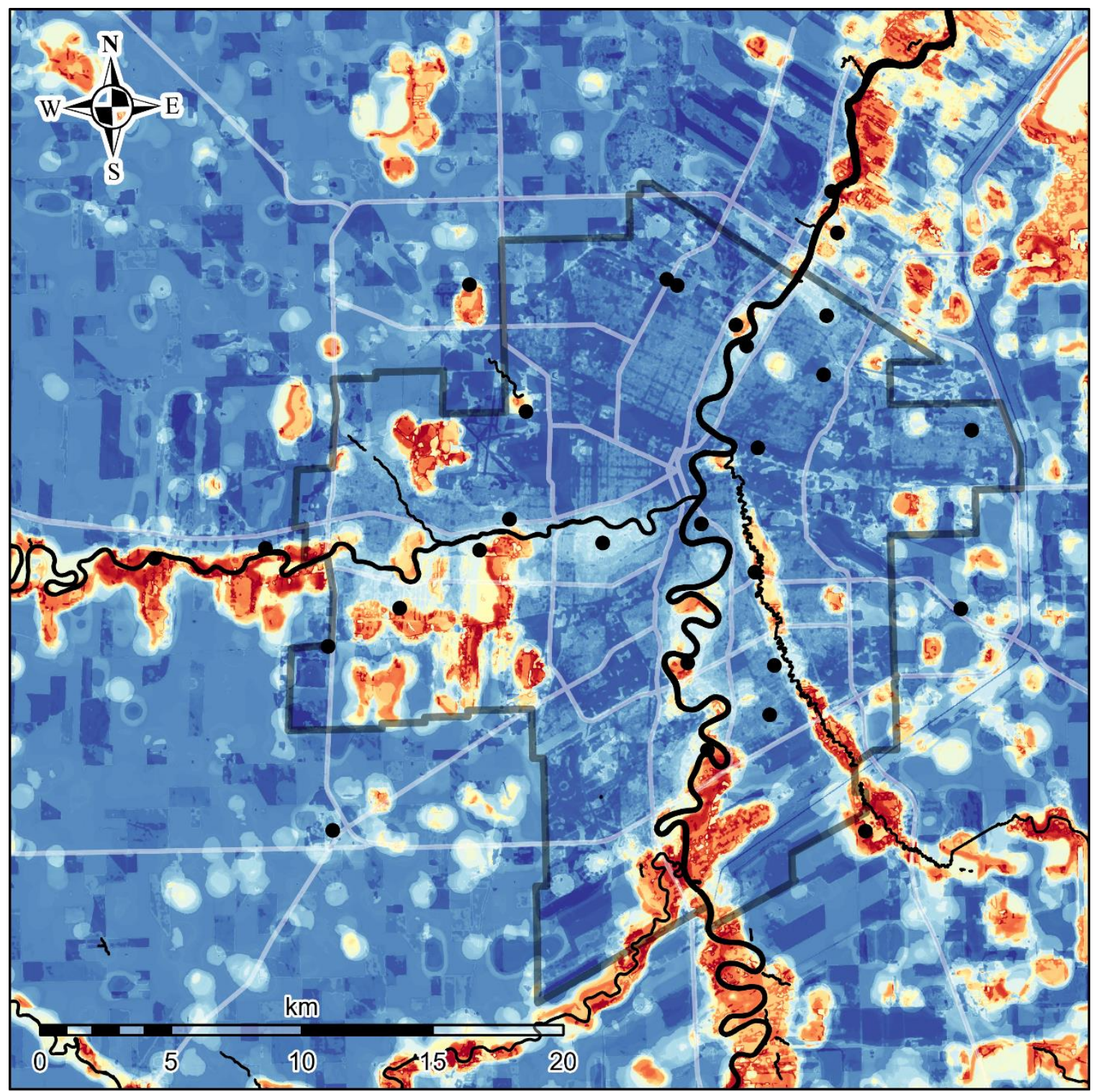

\section{Risk of Elevated Population for Aedes vexans ${ }^{a}$}

\section{LoW}

High

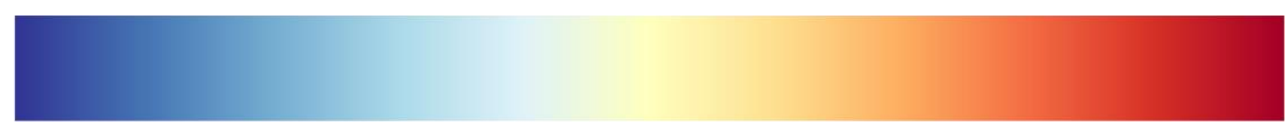

a Modeled using year-weighted trap indices

Figure 28. Risk map generated using a Random Forest regression to model the relative distribution of Aedes vexans in the Winnipeg region. Markers indicate source data trap locations, where representative trap counts were derived from the year-weighted trap index (TI). Winnipeg city limits and major roadways are outlined in black and white, respectively. 


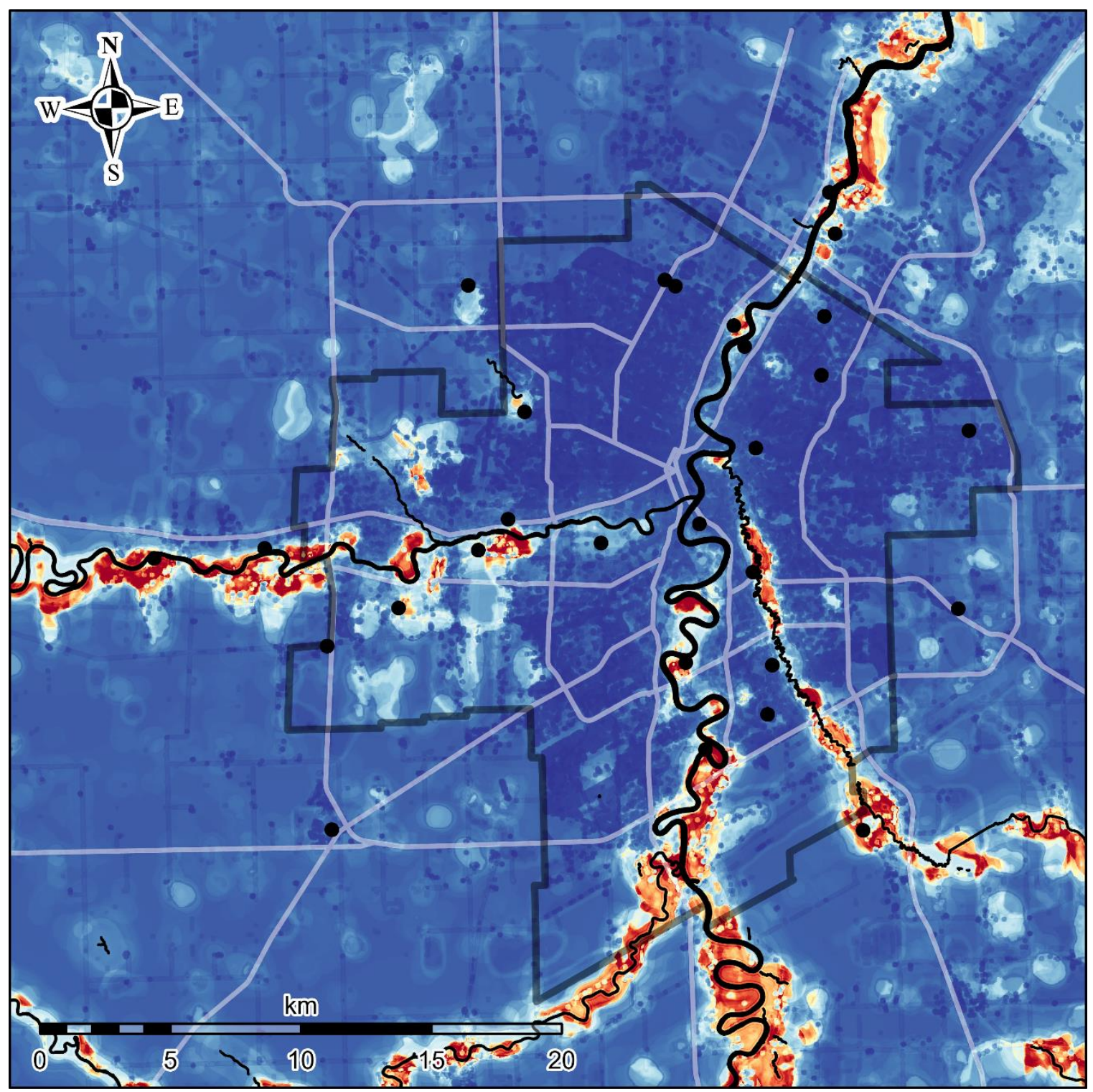

\section{Risk of Elevated Population for Aedes vexans ${ }^{b}$}

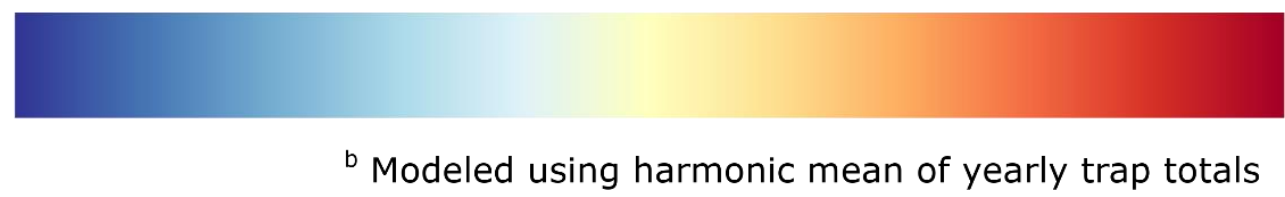

Figure 29. Risk map generated using a Random Forest regression to model the relative distribution of Aedes vexans in the Winnipeg region. Markers indicate source data trap locations, where representative trap counts were derived from the harmonic mean (HM) of yearly trap totals. Winnipeg city limits and major roadways are outlined in black and white, respectively. 
Table 16. Summary of best-performing Random Forest regression models for each mosquito species. The type of response variable used each model is indicated by the superscript next to the species name; TI stands for trap index, and HM for harmonic mean. The last column contains Kendall's tau $\left(r_{\tau}\right)$ correlation coefficient for each variable against its mosquito activity metric (e.g., species TI or HM).

\begin{tabular}{|c|c|c|c|c|c|c|}
\hline Species & MSE & $\begin{array}{c}\text { Variance } \\
\text { explained }(\%)\end{array}$ & Variable & $\begin{array}{c}\text { Effect } \\
\text { scale (m) }\end{array}$ & $\begin{array}{c}\text { Importance } \\
(\%)\end{array}$ & $\mathrm{r}_{\tau}$ \\
\hline \multirow[t]{4}{*}{ Ae. vexans ${ }^{T I}$} & 0.042 & 57 & Forest & 500 & 32 & 0.55 \\
\hline & & & NDVI & 50 & 24 & 0.29 \\
\hline & & & Grass & 500 & 22 & 0.16 \\
\hline & & & Residential & 1000 & 22 & -0.07 \\
\hline \multirow[t]{6}{*}{ Ae. vexans ${ }^{H M}$} & 0.280 & 45 & Forest & 500 & 27 & 0.52 \\
\hline & & & Distance to River & - & 22 & -0.28 \\
\hline & & & Commercial & 100 & 17 & -0.42 \\
\hline & & & Pavement & 100 & 12 & -0.27 \\
\hline & & & Grass & 500 & 12 & 0.16 \\
\hline & & & Residential & 500 & 9 & 0.01 \\
\hline \multirow[t]{8}{*}{ Cx. tarsalis $^{T I}$} & 0.060 & 53 & Grass & 500 & 21 & 0.41 \\
\hline & & & NDVI & 50 & 19 & -0.18 \\
\hline & & & Forest & 100 & 15 & -0.35 \\
\hline & & & Residential & 1000 & 14 & -0.40 \\
\hline & & & Ground & 250 & 9 & 0.28 \\
\hline & & & NDWI & 500 & 8 & 0.19 \\
\hline & & & River & 250 & 7 & -0.30 \\
\hline & & & Industrial & 100 & 6 & 0.19 \\
\hline \multirow[t]{7}{*}{ Cx. tarsalis ${ }^{H M}$} & 0.230 & 31 & Ground & 250 & 26 & 0.20 \\
\hline & & & Cultivated & 1000 & 24 & 0.29 \\
\hline & & & River & 1000 & 21 & -0.23 \\
\hline & & & Grass & 1000 & 17 & 0.32 \\
\hline & & & Commercial & 100 & 8 & -0.16 \\
\hline & & & Industrial & 50 & 3 & 0.14 \\
\hline & & & Water & 100 & 2 & 0.08 \\
\hline \multirow[t]{3}{*}{ Cx. restuans ${ }^{T I}$} & 0.069 & 37 & Residential & 500 & 46 & 0.26 \\
\hline & & & Forest & 500 & 42 & 0.35 \\
\hline & & & River & 250 & 11 & -0.07 \\
\hline \multirow[t]{5}{*}{ Cx. restuans ${ }^{H M}$} & 0.268 & 49 & Forest & 500 & 38 & 0.37 \\
\hline & & & Distance to River & - & 22 & -0.04 \\
\hline & & & River & 250 & 14 & -0.14 \\
\hline & & & Commercial & 100 & 13 & -0.24 \\
\hline & & & Ground & 100 & 13 & 0.13 \\
\hline \multirow[t]{3}{*}{ Ae. dorsalis ${ }^{T I}$} & 0.082 & 32 & Residential & 1000 & 46 & -0.38 \\
\hline & & & Cultivated & 1000 & 33 & 0.30 \\
\hline & & & Water & 500 & 21 & -0.14 \\
\hline \multirow[t]{2}{*}{ Ae. dorsalis ${ }^{H M}$} & 0.092 & 38 & Cultivated & 500 & 57 & 0.05 \\
\hline & & & Grass & 1000 & 43 & 0.18 \\
\hline
\end{tabular}




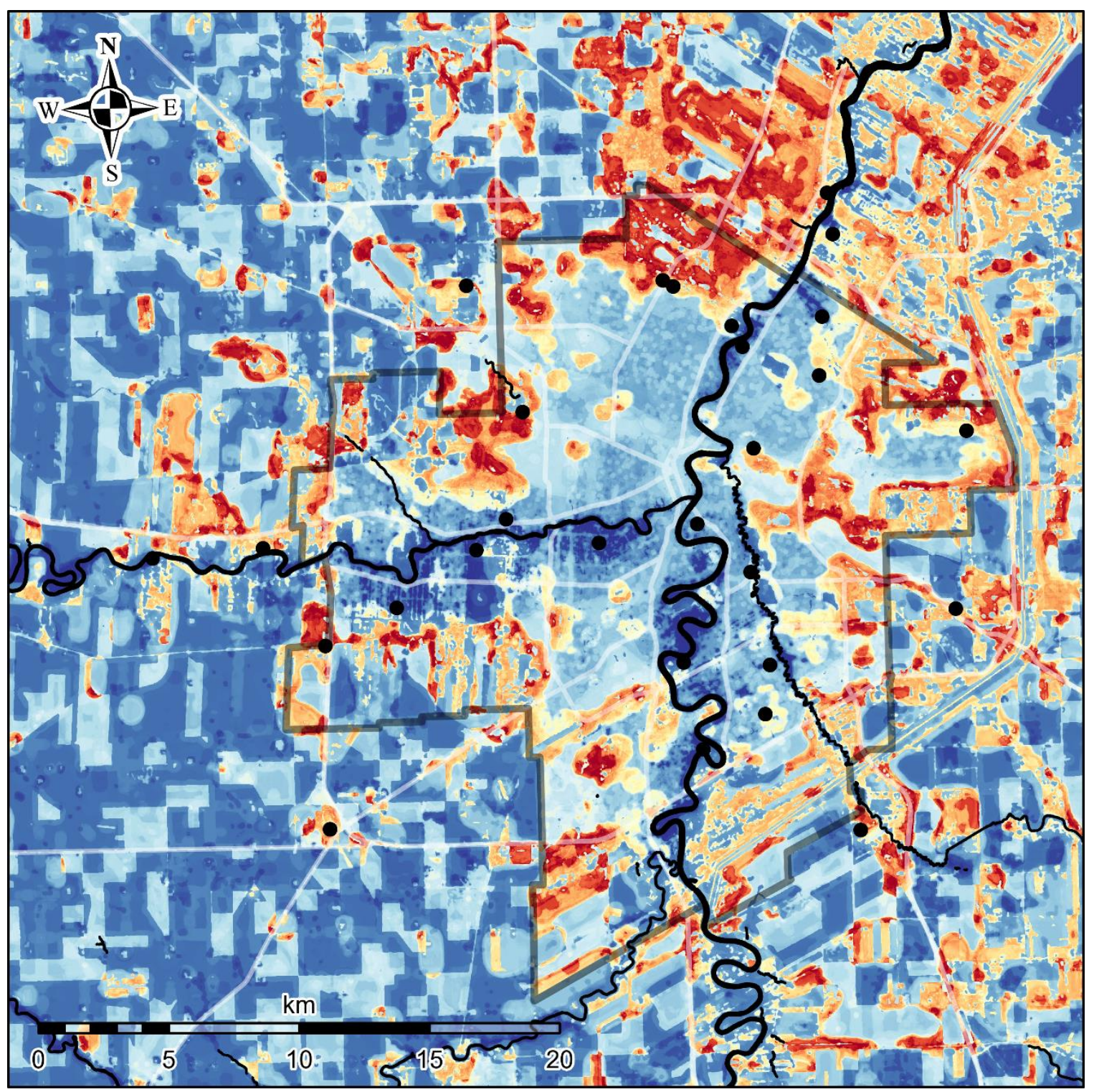

\section{Risk of Elevated Population for Culex tarsalis ${ }^{a}$}

\section{Low}

High

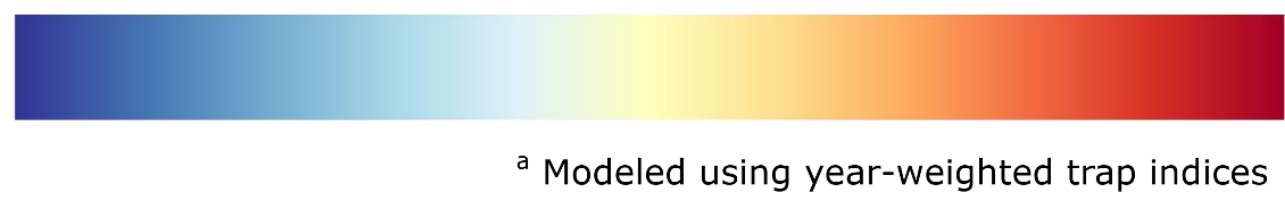

Figure 30. Risk map generated using a Random Forest regression to model the relative distribution of Culex tarsalis in the Winnipeg region. Markers indicate source data trap locations, where representative trap counts were derived from the year-weighted trap index (TI). Winnipeg city limits and major roadways are outlined in black and white, respectively. 


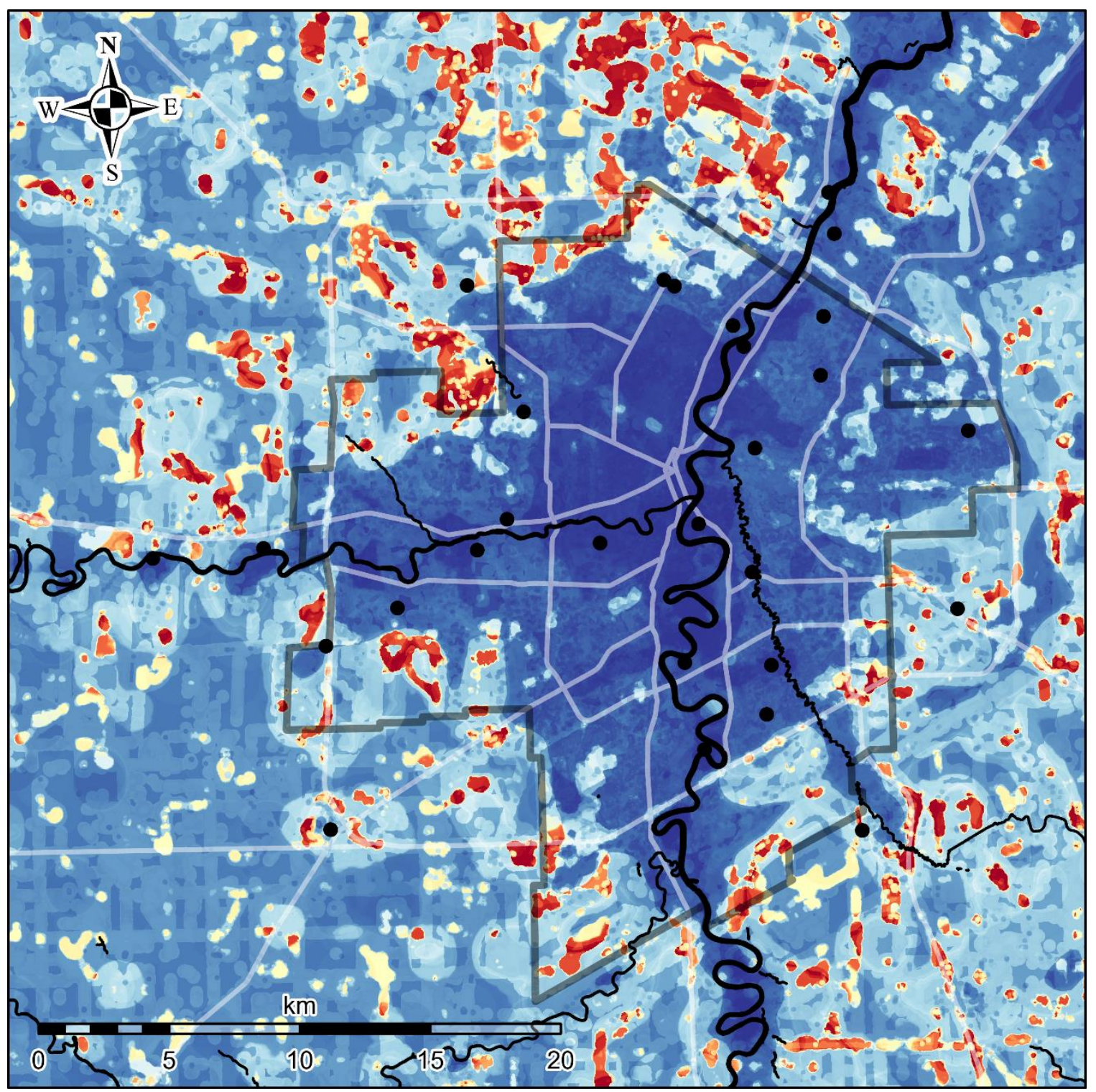

\section{Risk of Elevated Population for Culex tarsalis}

\section{Low}

High

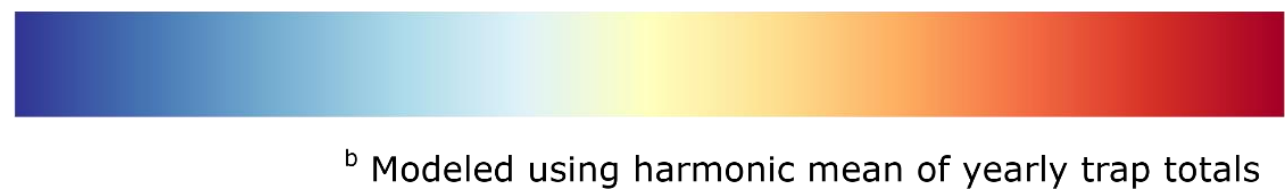

Figure 31. Risk map generated using a Random Forest regression to model the relative distribution of Culex tarsalis in the Winnipeg region. Markers indicate source data trap locations, where representative trap counts were derived from the harmonic mean (HM) of yearly trap totals. Winnipeg city limits and major roadways are outlined in black and white, respectively. 


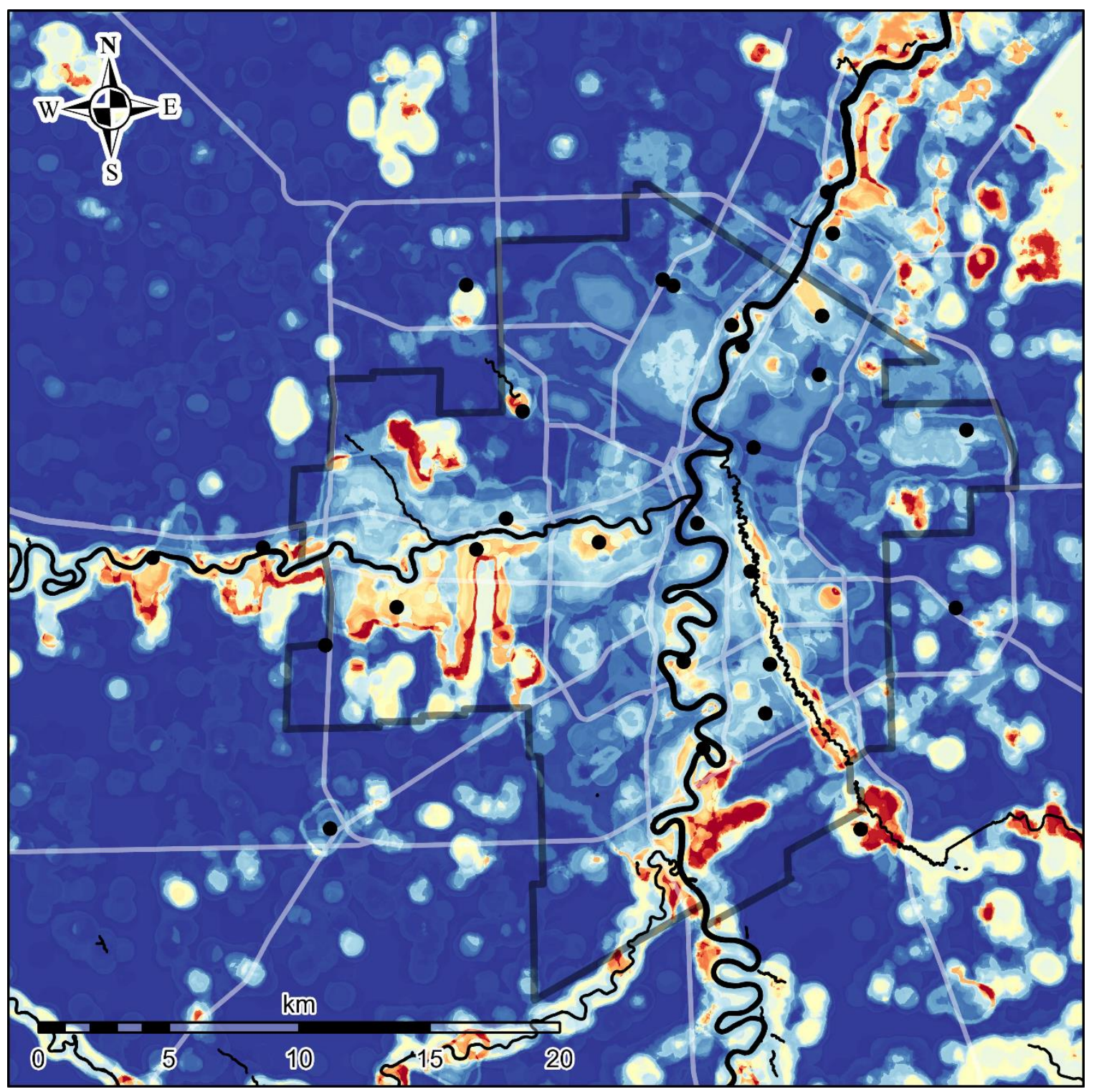

\section{Risk of Elevated Population for Culex restuans ${ }^{a}$}

\section{${ }^{a}$ Modeled using year-weighted trap indices}

Figure 32. Risk map generated using a Random Forest regression to model the relative distribution of Culex restuans in the Winnipeg region. Markers indicate source data trap locations, where representative trap counts were derived from the year-weighted trap indices (TI). Winnipeg city limits and major roadways are outlined in black and white, respectively. 


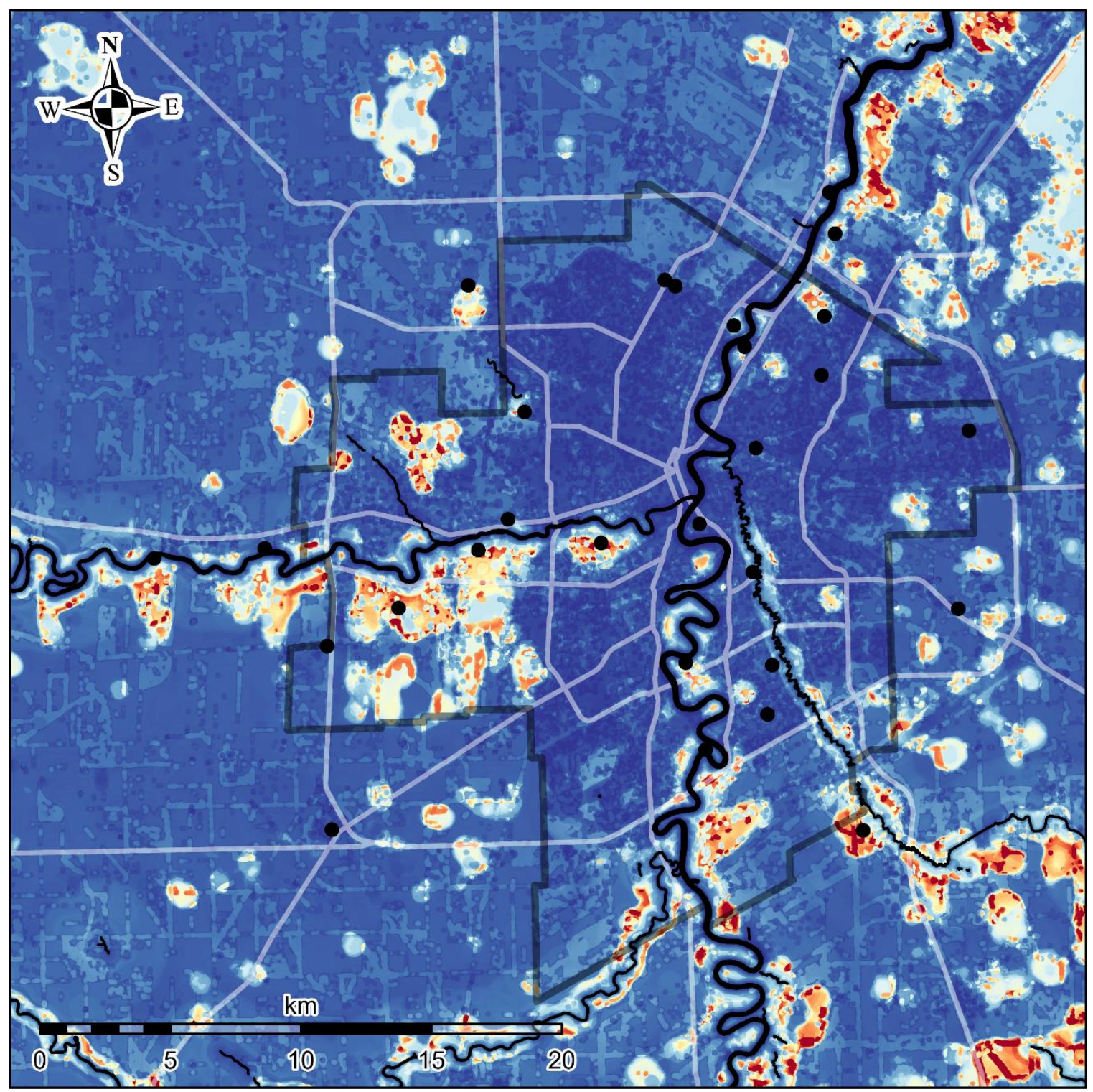

\section{Risk of Elevated Population for Culex restuans ${ }^{\mathrm{b}}$}

\section{Low}

High

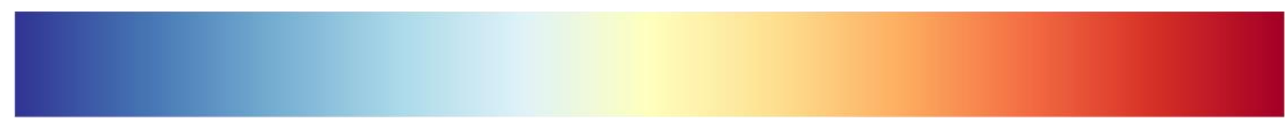

${ }^{\mathrm{b}}$ Modeled using harmonic mean of yearly trap totals

Figure 33. Risk map generated using a Random Forest regression to model the relative distribution of Culex restuans in the Winnipeg region. Markers indicate source data trap locations, where representative trap counts were derived from the harmonic means (HM) of yearly trap totals. Winnipeg city limits and major roadways are outlined in black and white, respectively. 


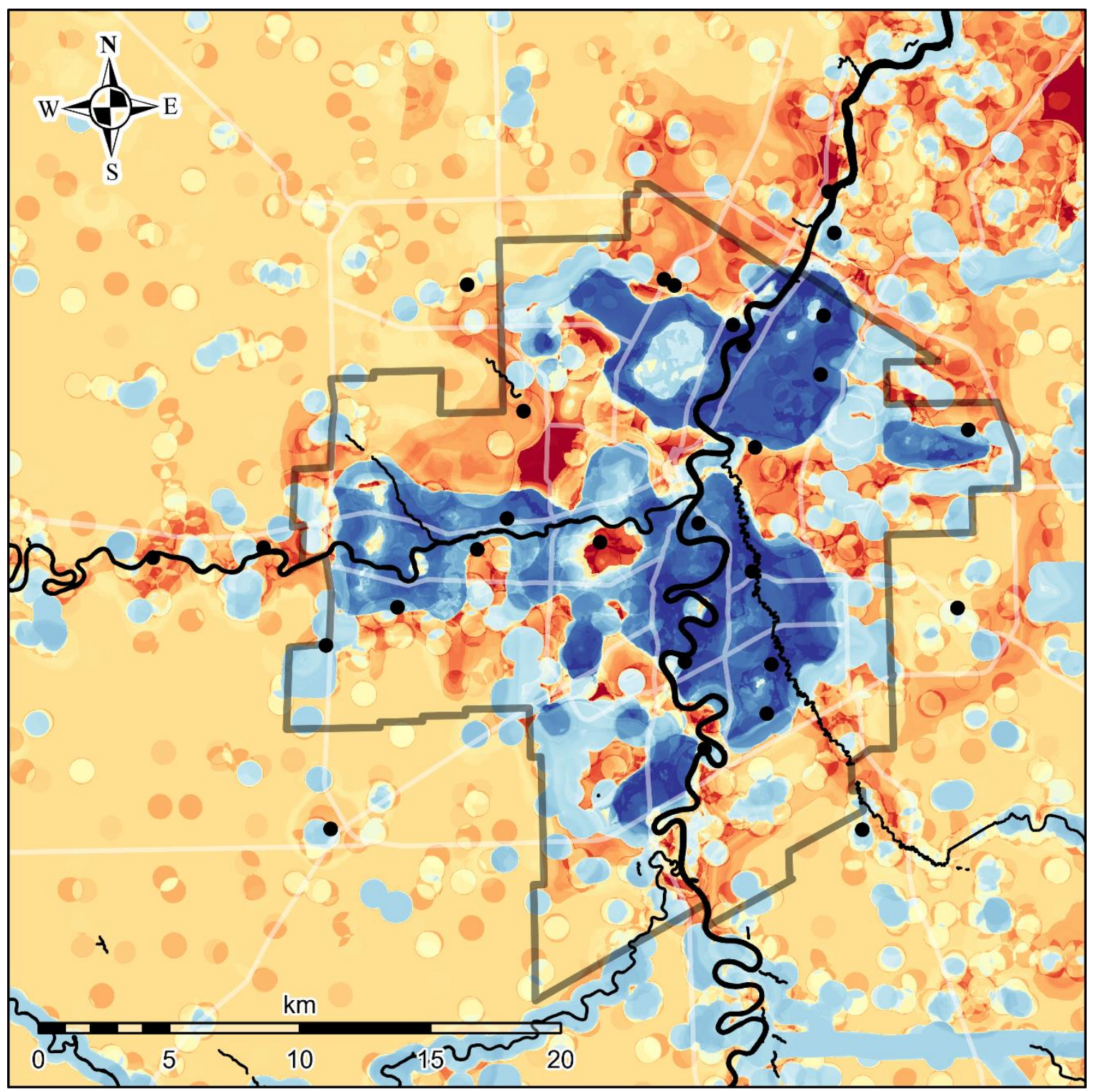

\section{Risk of Elevated Population for Aedes dorsalis}

\section{a Modeled using year-weighted trap indices}

Figure 34. Risk map generated using a Random Forest regression to model the relative distribution of Aedes dorsalis in the Winnipeg region. Markers indicate source data trap locations, where representative trap counts were derived from the year-weighted trap indices (TI). Winnipeg city limits and major roadways are outlined in black and white, respectively. 


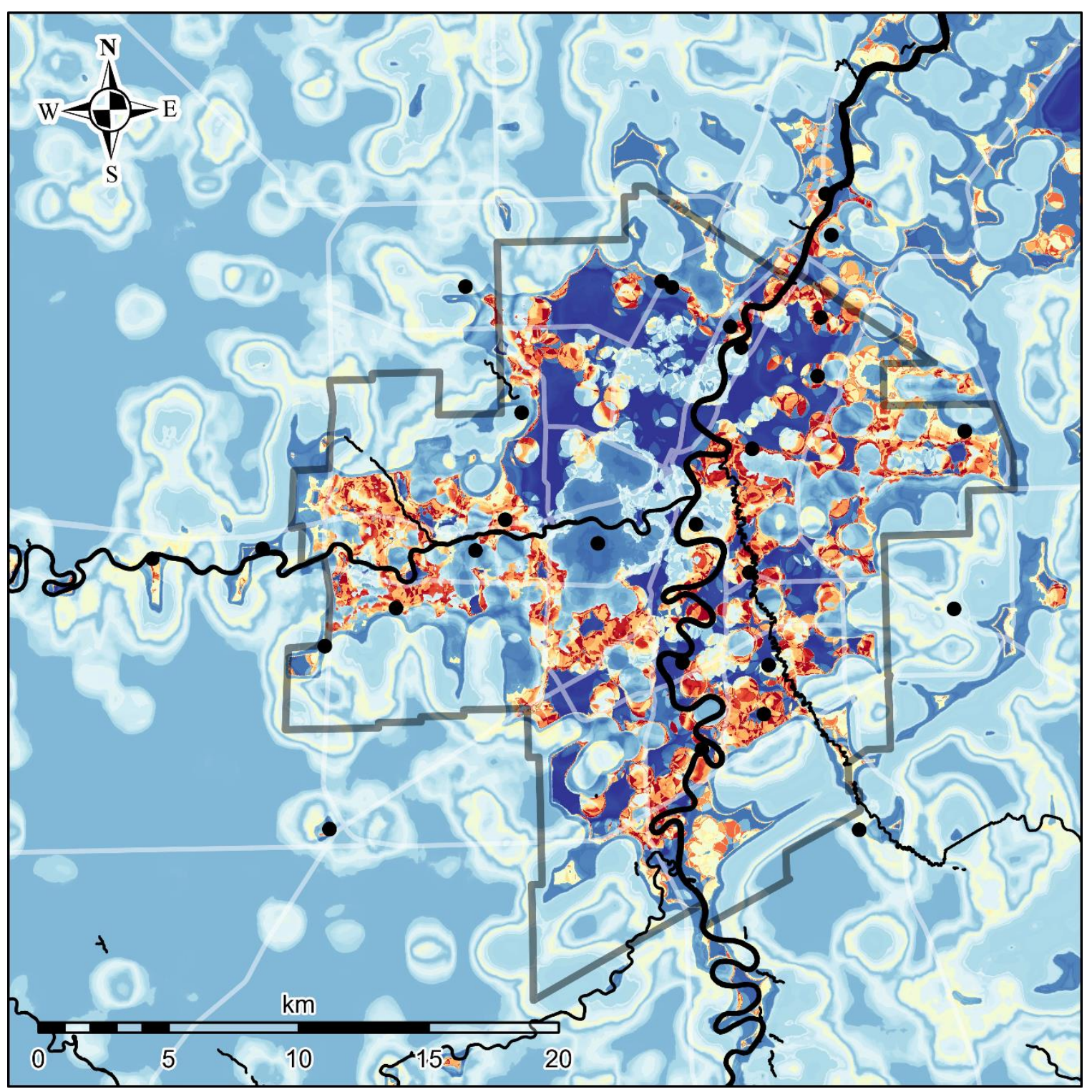

\section{Risk of Elevated Population for Aedes dorsalis ${ }^{b}$}

\section{${ }^{\mathrm{b}}$ Modeled using harmonic mean of yearly trap totals}

Figure 35. Risk map generated using a Random Forest regression to model the relative distribution of Aedes dorsalis in the Winnipeg region. Markers indicate source data trap locations, where representative trap counts were derived from the harmonic means (HM) of yearly trap totals. Winnipeg city limits and major roadways are outlined in black and white, respectively. 
or a relatively low risk (Figure 35) of elevated mosquito activity. In the TI-based model, few hot spots were identified for Ae. dorsalis populations. Most of the region outside of the city limits posed average to relatively higher risk in comparison to the inner city. With the HM-based model, hotspots were patchy and located throughout the inner city.

\section{4 - Discussion}

\subsection{1 - Object-based LULC classification}

Given that kappa is intended to be used in situations where all classes have equal sample sizes (Congalton 1991), the kappa value obtained in the landscape classification may be artificially low because of unbalanced training data. The extent of the thematic LULC map was the combined area within $4 \mathrm{~km}$ of each trap and was larger than the area used to generate the training data. Since the $4 \mathrm{~km}$ radii surrounding the traps (correcting for overlapping area) amounted to $727 \mathrm{~km}^{2}$, this meant that the training samples represented less than $40 \%$ of the total study area. Therefore, there were fewer training samples for cultivated land than would have been if the entire study extent had been used to generate random sample points for this task. This was despite cultivated land cover having had the greatest number of training samples. As the study area was comprised of approximately $25 \%$ urban or peri-urban area and $75 \%$ cultivated land in its $\sim 2000 \mathrm{~km}^{2}$ scope. This introduced bias in the classification process.

It follows that the classifier assumed less cultivated land would be present across the study area than was present on the landscape. Subsequently, the producer's accuracy (56\%) saw that the classifier often misidentified cultivated land as grassy open area or forest, assuming these classes to occupy more area than they do (Table 14). Conversely, commercial and industrial areas were overrepresented in area among the training samples despite (again) having the fewest samples. This led the classifier to misclassify them as other related (or frequently co-present) classes, which led to lower user's accuracies (54 to 58\%), as is common in image classification studies (Werner et al. 2014). For example, pavement and residential areas were erroneously zoned as commercial area, and ground was often mistaken for industrial land. 
However, this was generally not an issue in the characterization of trap surroundings, as these misclassified areas were relatively small compared to the correctly identified areas that surrounded them. Traps with substantial cultivated land area surrounding them still reported large proportions of cultivated land in their surroundings, with negligible effect to the overall proportion of grass and forest cover reported. Admittedly, this may have led to small effects in variable importance within the final models. Regardless, the calculated Kappa coefficient indicated an acceptable degree of agreement between the classifier and the reference data. Therefore, I feel that that thematic LULC map generated was sufficiently accurate for the task of risk mapping, specifically where the risk drivers are multi-factorial in nature.

\subsection{2 - Moran's I}

As expected, trap count values were not spatially autocorrelated for any species. This was likely because the traps were located too far apart to represent the true spatial heterogeneity of the urban Winnipeg area where most traps were located.

\subsection{3 - Spatial distribution models}

It was also expected that Ae. vexans distribution models would perform better than the other models on average, as effects are easier to detect with larger samples (Krzywinski and Altman 2013), and Ae. vexans represented over 95\% of mosquitos collected by the source traps. The clustering of their distribution around rivers corroborates what is already known about Ae. vexans as floodwater mosquitoes (O’Malley 1990; Becker 2010); females are known to lay their eggs in moist soils, with Ae. vexans showing preference for areas where the water table is high. Many geospatial studies echo this, in finding that Ae. vexans are associated with proximity to wetlands (Moncayo et al. 2000; Trawinski and Mackay 2010; Giordano et al. 2018) and surface wetness (Shaman et al. 2002; Chuang, Hockett, et al. 2012).

While both Ae. vexans models reported slightly different variables as important predictors of mosquito activity, their mapped distributions were similar regardless of the mosquito activity metric used. The surrounding presence of forested areas was the most important predictor in both models which reflects 1) the mosquito as an evening-feeder 
that inhabits shady understory vegetation during the day (Bidlingmayer 1971; Mullen 1971; Burkett-Cadena et al. 2013), and 2) the close association with forests and rivers in the Winnipeg area. While only the HM-based model reported a river-related metric as a predictor, the TI-based model identified NDVI within the immediate vicinity of the trap as a key predictor. This may function as a proxy for riparian areas near rivers within this model, given that NDVI is correlated with primary productivity, and riparian areas are among the most productive ecosystems in part because of their high water tables (United States Department of Agriculture 1996). The inclusion of grass and residential areas as a predictor in both models may also follow this inference, as residential vegetation such as lawns may mimic the moist soil in which Ae. vexans oviposit.

The negative correlations between mosquito activity, and pavement and commercial areas within the HM-based Ae. vexans model appears to have restricted the distribution of elevated populations into residential areas surrounding the rivers. Both models agree that the presence of riparian areas is important, but they disagree on the specificity of these habits and the extent to which residential areas may also harbour hot spots for Ae. vexans.

The risk projections for $C x$. tarsalis align with their tendency to occupy irrigated rural areas (Moore et al. 1993) and regions lacking in vegetation where standing water is most likely to pool in artificial containers capable of collecting rainwater (Wood et al. 1979). Both models list positive correlations with bare ground, open grasslands, and industrial areas. These results agree with most other GIS-based studies of $C x$. tarsalis activity, where we see correlation with non-forested or otherwise less productive areas (Diuk-Wasser et al. 2006; Yoo et al. 2016), as well as cultivated and grassland regions (Nielsen et al. 2008; Chuang et al. 2011). Their preference for polluted or eutrophic standing water may be enabled by cultivated or industrial land use, which concentrate organic nutrients in dry conditions (Shaman et al. 2002; Winters et al. 2008; Eisen et al. 2010). This also explains the inclusion of river area as a negative-associated predictor in both $C x$. tarsalis models, as these areas would be the least likely to be eutrophic.

Like the Ae. vexans models, the $C x$. tarsalis models also generally agree on the major drivers of mosquito activity but disagree in terms of determining whether these 
encroach on urban areas outside of industrial areas. Again, the HM-based model gives more specificity with respect to its risk projections for $C x$. tarsalis.

For $C x$. restuans, the areas with greatest predicted risk of elevated were predicted to be near forested areas in the vicinity of, but not in the immediate proximity (within 250 m) of, a river, unlike Ae. vexans. This agrees with their affinity for shaded oviposition sites under dense tree canopies, where they occupy a variety of habitats, such as ditches, stream pools and artificial containers (Walter Reed Biosystematics Unit 2020). Given that riverrelated metrics appear to be only weakly correlated with $C x$. restuans activity, but improve model performance, it may be that they mediate the effects of interactions involving themselves and other predictors. To my knowledge, this is the first attempt to model $C x$. restuans activity in an urban area using GIS-based analysis.

The distribution models for Ae. dorsalis also represent a first for the species. However, these models performed the poorest out of all models regardless of species. While cultivated land area (within $1 \mathrm{~km}$ ) was a strong predictor of activity in both models, its relationship with Ae. dorsalis populations are not clear, given discrepancies in model projections. As this species only contributes $>1 \%$ of specimens in all collections from the study period, it may be that their sample size is not sufficiently large to detect an effect. Additionally, TI and HM values were most dissimilar for Ae. dorsalis, where the three trap locations ranked highest for activity by TI did not match those same rankings by HM (Appendix XVII).

\subsection{4 - Use of TI and HM}

The inconsistency in performance between the TI and HM-based models is not obvious in its origin but may be related to relative variation across yearly trap counts. At face value, HM-based models appeared to perform better in explaining variance in $C x$. restuans and Ae. dorsalis activity (49 and 38\%, respectively), whereas TI-based models explained more variance for Ae. vexans and Cx. tarsalis (57 and 53\%, respectively). An overview of the total yearly trap counts for each trap revealed that this may be related to the average yearly variation among species as indicated by the coefficient of variation $(\mathrm{CV})$. The $\mathrm{CV}$ is a measure of relative variability between multiple datasets with different measures or values. It is calculated by dividing the sample standard deviation by the sample 
mean (Glen, n.d.). When calculating CV using the proportion of the total yearly number of mosquitoes collected per trap (used in calculating TI), the overall coefficient of variation of the proportions of Ae. dorsalis collected in individual traps each year (0.76) was more than double than that of Ae. vexans, Cx. tarsalis, and Cx. restuans $(0.30,0.32$, and 0.35 , respectively; see Appendix XVIII). In other words, the yearly variation between individual traps varies less for Ae. vexans, $C x$. tarsalis, and $C x$. restuans than the yearly trap variation for Ae. dorsalis. Similarly, when calculating CV for the HM values, which used the inverse of the total number of mosquitoes caught per trap each year, I found that all other species demonstrated lower overall variability between years in comparison to Ae. dorsalis (see Appendix XIX). While this may explain relatively low model performance in general with Ae. dorsalis, it is still unclear where the unusual discrepancy in performance between TIbased and HM-based models for $C x$. restuans originated. In the TI-based models, $C x$. restuans had total yearly $\mathrm{CV}$ that was lower than that of Ae. dorsalis, but close in value to the two other species. However, this trend was not repeated in the yearly CVs for the HMbased models, as $C x$. restuans had the lowest variability overall.

This infers that consideration must be given to multi-year trends in activity before selecting a metric to represent it as a response variable and an analytical tool such as RF. A higher coefficient of variation between years may indicate unstable or more stochastic population emergences, which may be better explained by HM-based models. Aedes dorsalis had the highest overall coefficient of variation in both models, which both performed poorly overall, but the HM-based model outperformed the TI-based model for this species. Due to the way that a RF model's percent variance explained is calculated (see Ch. 2), a model where the response variable exhibits greater variation will generally result in a better performance than that with less variance, if all other factors are equal. However, it is not clear what other factors contribute to differences between TI and HM-based models. Higher performance in the HM-based model was not corroborated by a higher overall coefficient of variation for $C x$. restuans. 


\subsection{5 - Conclusions}

Overall, the effect scales showed different trends depending on the predictors selected by the models. The amount of surrounding vegetation-based land cover classes such as cultivated land, grass and forest tended to be most effective at 500 to $1000 \mathrm{~m}$ across all models, whereas certain land use classes such as commercial and industrial areas were more often influential at a 50 to $100 \mathrm{~m}$ scale. This may reflect the average size of these areas on the landscape, where swaths of forested or cultivated land tend to be larger than industrial and commercial areas. Regardless, this demonstrates that relationships between mosquitoes and their surroundings extend beyond the reach of their olfactory or visual senses.

The analytical procedure presented here serves as an example of a user-friendly approach to spatial modelling using data from traps. The utility of TI and HM as activity metrics may be limited to studies with multiple years of data, but the extraction of spatial data in various zonal area sizes combined with the use of non-linear trend analysis (e.g., Random Forest) leads to more robust results. The use of machine learning can be used to produce risk maps that are easily interpretable without familiarity with the details of the study. While the RF models could be further validated by trap data from other years, its robustness is demonstrated by the consistency of the predictors and their effective scales observed in predictor variables between TI and HM-based models. While there is no precedent in the literature for use of TI and HM as metrics of mosquito activity, their use is recommended if a representative value for many groups is required, especially where sample sizes among groups vary on a logarithmic scale.

While TI and HM were used here as metrics of mosquito activity, this was only to address the structure of the dataset. Predictions of mosquito distributions during more recent or more specific surveillance periods could be generated using only monthly or weekly mosquito trap counts as well.

I also recommend developing models with multiple scales for each predictor variable in the dataset. When measuring the collinearity of the same variables over multiple scales, I found that collinearity was low. This indicates that a significant amount of spatial heterogeneity was added to the landscape with each increase in the scale of the trap 
environment from which spatial data were extracted. The radii for extracting information about trap environments were chosen arbitrarily in these analyses but reflect both the largest size possible before numerous zonal areas intersect and the previously documented flight capabilities for the most common mosquito species. However, we encourage others to try including information extracted from trap environments at greater scales, as these analyses have demonstrated that mosquito may be influenced by their surrounding landscape on a much larger scale than previously thought.

Finally, while the RF procedure that was used to predict mosquito activity in unsampled areas works well on small samples (only 28 traps were sampled, 30 are recommended by ArcGIS Pro), we caution that it not be used to predict the distribution of species for which there are relatively few specimens. Compared to the other three species, the models for Ae. dorsalis were built upon fewer specimens spread across fewer collection events, with many traps collecting no individuals some years. Lack of variation in the data produced the poorest models and distribution patterns that were not replicated when a different activity metric (HM or TI) was used. The models produced by the methods given in Ch. 4 may be incompatible with most procedures used to develop species distribution models for endangered populations, for which activity is usually much lower and less variable.

The most successful model in this study was that built using surveillance data from Winnipeg's most common mosquito species, Ae. vexans, which was notably present in every trap in ever year, with greatest variation in their numbers. For those intend to model mosquito distribution in the future: it may be worthwhile to ponder the structure of datasets where presence is the rule, not the exception, as it may not be appropriate to approach these with the same modelling techniques used for species at-risk analyses.

These findings may represent opportunities for improved mosquito control efforts. As mosquito activity risk is presented in $10 \mathrm{~m}$ resolution, it is possible to use these risk maps to inform nuanced control strategies within neighbourhoods based on the risk predicted by their unique environmental characteristics (Figure 36). Given that these risk maps are highly interpretable by non-experts, they also present valuable tools for communicating control program operations with the public. 

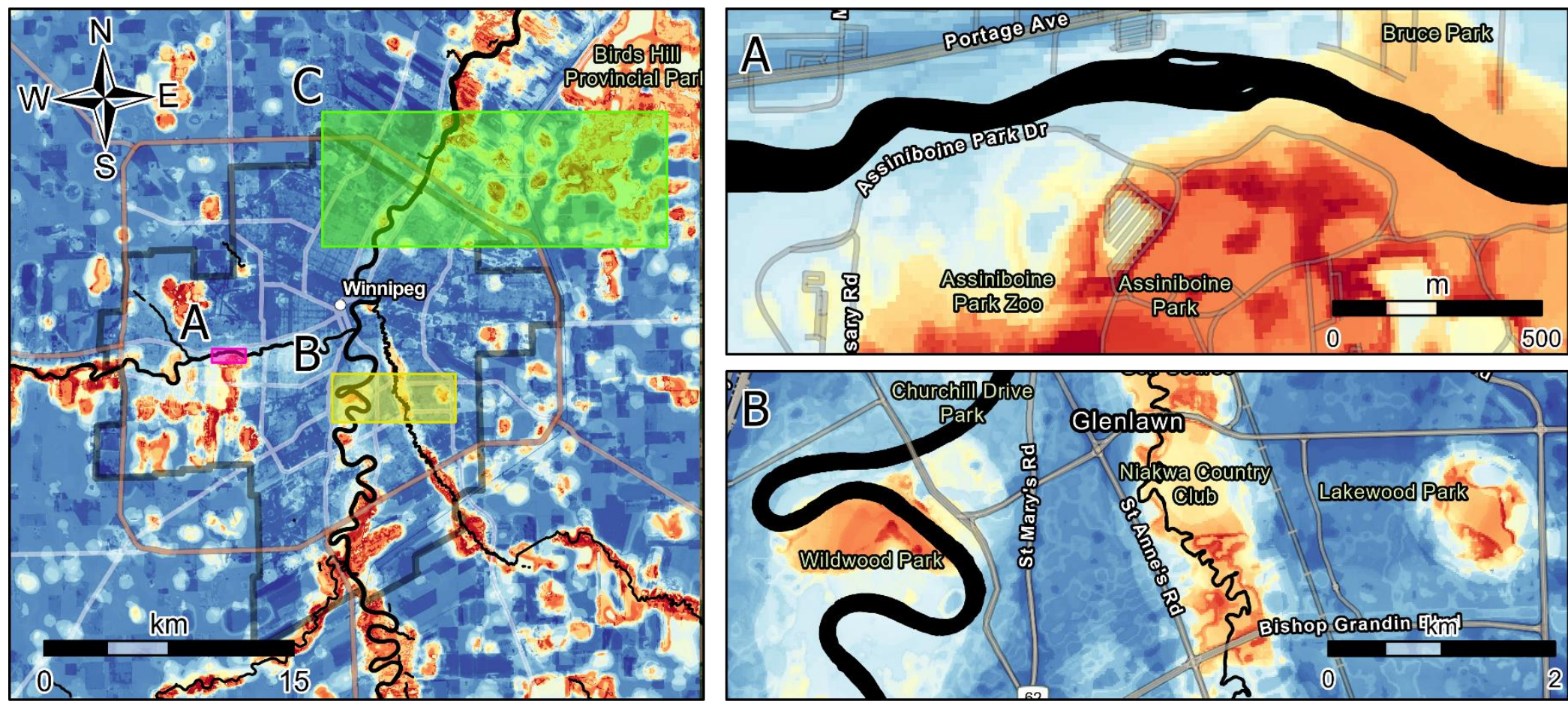

Risk of elevated population for Ae. vexans ${ }^{\mathrm{a}}$

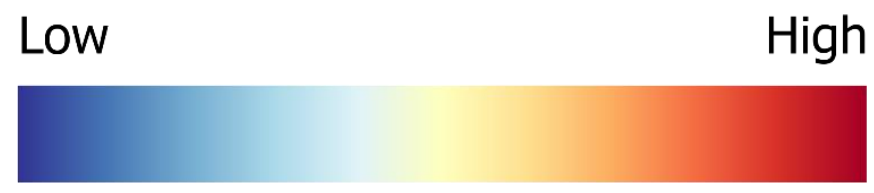

a Modeled using harmonic mean of yearly trap totals

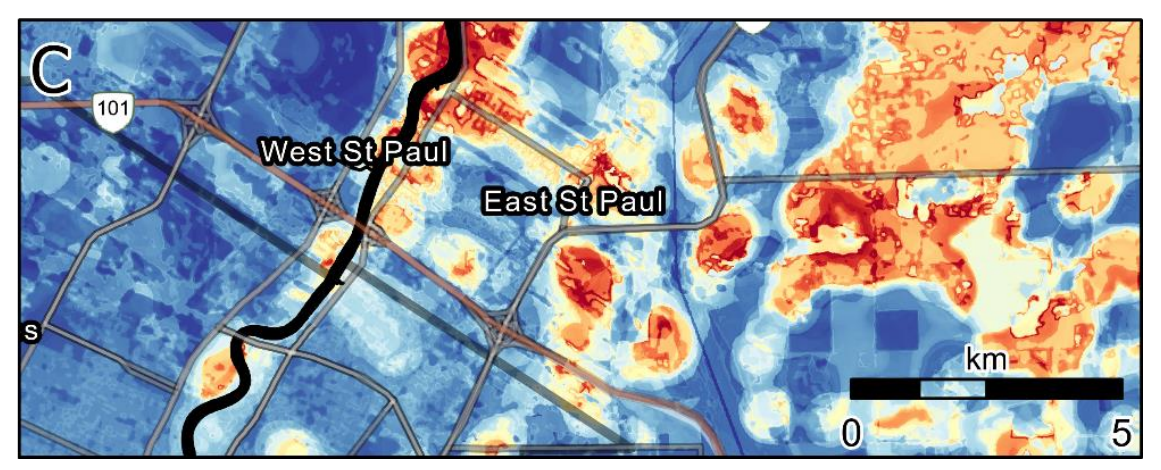

Figure 36. Risk maps for Aedes vexans at neighbourhood scale (10 $\mathrm{m}$ resolution). 


\section{CHAPTER 5 - OVERALL DISCUSSION}

A comparison of the outcomes of the MRR studies (Ch. 3) and spatial analyses (Ch. 4) revealed complementary findings with respect to the behaviour of both Aedes vexans (Meigen, 1830) and Cx. tarsalis Coquillett, 1896, as well as the usefulness of a mosquito control buffer zone extending $8 \mathrm{~km}$ or more beyond Winnipeg city boundaries.

The floodwater mosquito Ae. vexans was consistently demonstrated in MRR experiments to have strong flight capabilities. They were also shown to prefer areas with more vegetative cover and higher humidity, having been recaptured more often in forested areas, many of which were near to natural rivers like the Assiniboine and the Red. In the spatial analyses, the areas at greatest risk of developing elevated populations of Ae. vexans were those surrounding rivers or that were otherwise high in vegetation. It is tempting to assume that since over $95 \%$ of mosquitoes captured in the traps in these experiments were Ae. vexans, that ICB should prioritize their control efforts on riparian areas. However, it is worthwhile to note briefly that these studies are biased toward mosquitoes that can be sampled using unbaited NJLTs, which may not have been a representative sample of all mosquitoes in this area. Additionally, since NJLTs primarily attract mosquitoes using light, they are more likely to be caught at night. This means that their daytime distribution may differ from their nighttime distribution. Further studies are required to determine if $A e$. vexans are more likely to be caught at night as a resulting of their movement out of riparian areas into nearby residential areas with potential hosts.

Regardless, we can speculate that Ae. vexans, and possibly Culex restuans as well, are using riverine corridors for dispersal. Colonization by immigrant mosquito populations, including invasive species of Aedes mosquitoes, are likely to occur via these corridors. Concerning the maintenance of mosquito control operations in the buffer zone surrounding the city, strategic application of larvicides or ULV application at possible mosquito entry points (e.g., where each tributary to the Red River enters the urban area) may be an effective means of suppressing the establishment of new mosquito populations. Alternatively, if there are resources to regularly identify all mosquito species sampled at these points, this may be the best way to detect an invasive species early. 
While there were only four $C x$. tarsalis mosquitoes recaptured during both MRR study years, three of these were recaptured at traps where there was little vegetative cover. This corroborates the conclusions inferred by the spatial analyses on the distribution of $C x$. tarsalis, a West Nile virus vector, where models predicted that their activity level would be correlated with larger areas of bare ground, grass or cultivated land cover. This suggests that artificial containers and undrained areas, rather than floodplains, may pose the biggest hazards for development of high $C x$. tarsalis populations. As $5 \mathrm{~km}$ was the upper limit of Cx. tarsalis recaptures in the MRR studies of 2016 and 2017, it is the minimum width for suppression of $C x$. tarsalis populations within Winnipeg.

Overall, the MRR experiments and spatial analyses highlight a mosquito abatement challenge with respect to the usefulness of the buffer zone. As Ae. vexans and $C x$. tarsalis are currently the primary focus of mosquito control efforts in Winnipeg, all potential larval habitats within the buffer zone would require treatment or removal to prevent large scale dispersal into the city. This would necessitate comprehensive treatment or removal of all possible larval habitats for these two species. Given the difference in habitat preference seen in the risk maps generated for Ae. vexans and $C x$. tarsalis, this would call for resources likely beyond what is available.

Further problems arise when we consider intricacies of their life cycles; Ae. vexans habitats are not always identifiable unless larvae are developing, as soils where female oviposit only become larval habitats when periodically flooded. Similarly, many of the artificial containers used by $C x$. tarsalis as larval habitats are only available when enough standing water accumulates within them. Access to potential larval habitats on private property, as well as difficulty detecting cryptic habitats (e.g., small ephemeral puddles and containers) with current technology creates further challenges for mosquito control efforts intending to treat all larval habitats within the buffer zone. While these studies allow for an improved understanding of mosquito ecology in the Winnipeg area, their usefulness for ICB in making control decisions is not yet clear. However, local knowledge of habitats preferred by nuisance and vector mosquitoes can aid in improving trap coverage to enhance surveillance. 


\section{REFERENCES}

Adam E, Mutanga O, Odindi J, Abdel-Rahman EM. 2014. Land-use/cover classification in a heterogeneous coastal landscape using RapidEye imagery: evaluating the performance of random forest and support vector machines classifiers. Int J Remote Sens. 35(10):3440-3458. doi:10.1080/01431161.2014.903435.

Ageep TB, Damiens D, Alsharif B, Ahmed A, Salih EHO, Ahmed FTA, Diabaté A, Lees RS, Gilles JRL, El Sayed BB. 2014. Participation of irradiated Anopheles arabiensis males in swarms following field release in Sudan. Malar J. 13(1):1-11. doi:10.1186/14752875-13-484.

American Mosquito Control Association. 2017. Best Practices For Integrated Mosquito Management: A Focused Update.

Anderson JR, Hardy EE, Roach JT, Witmer RE. 1976. A Land Use and Land Cover Classification System for Use with Remote Sensor Data. Geol Surv Prof Pap. 694.

Anderson RA, Gallaway WJ. 1987. The host preferences of Culiseta inornata in southwestern Manitoba. J Am Mosq Control Assoc. 3(2):219-221.

Asrar G, Fuchs M, Kanemasu ET, Hatfield JL. 1984. Estimating absorbed photosynthetic radiation and leaf area index from spectral reflectance in wheat. Agron J. 76(2):300-306. doi:10.2134/agronj1984.00021962007600020029x.

Ba Y, Diallo D, Mouhamed C, Kebe F, Diallo M, Dia I. 2005. Aspects of bioecology of two Rift Valley fever virus vectors in Senegal (West Africa): Aedes vexans and Culex poicilipes (Diptera: Culicidae). J Med Entomol. 42(5):739-750.

Bailey SF, Eliason DA, Hoffmann BL. 1965. Flight and dispersal of the mosquito Culex tarsalis Coquillett in the Sacramento Valley of California. Hilgardia. 37(3):73-113.

Barber LM, Schleier JJ, Peterson RKD. 2010. Economic cost analysis of West Nile virus outbreak, Sacramento County, California, USA, 2005. Emerg Infect Dis. 16(3):480-486. doi:10.3201/eid1603.090667.

Barr RA, Smith TA, Boreham MM. 1960. Light intensity and the attraction of mosquitoes to light traps. J Econ Entomol. 53(5):876-880.

Barr RA, Smith TA, Boreham MM, White KE. 1963. Evaluation of some factors affective the efficiency of light traps in collecting mosquitoes. J Econ Entomol. 56(2):123-127.

Barrett AD. 2014. Economic burden of West Nile virus in the United States. Am J Trop Med Hyg. 90(3):389-390.

Becker N. 2010. Mosquitoes and Their Control. 2nd ed. Springer.

Beier JC, Berry WJ, Craig GB. 1982. Horizontal distribution of adult Aedes triseriatus (Diptera:Culicidae) in relation to habitat structure, oviposition, and other mosquito species. J Med Entomol. 19(3):239-247.

Bell JR, Bohan DA, Shaw EM, Weyman GS. 2005. Ballooning dispersal using silk: world 
fauna, phylogenies, genetics and models. Bull Entomol Res. 95:69-114.

Benedict MQ, Charlwood JD, Harrington LC, Lounibos LP, Reisen WK, Tabachnick WJ. 2018. Guidance for evaluating the safety of experimental releases of mosquitoes, emphasizing mark-release-recapture techniques. Vector-Borne Zoonotic Dis. 18(1):3948. doi:10.1089/vbz.2017.2152.

Benedict MQ, Levine RS, Hawley WA, Lounibos LP. 2007. Spread of the tiger: Global risk of invasion by the mosquito Aedes albopictus. Vector Borne Zoonotic Dis. 7(1):7685. doi:10.1055/s-0029-1237430.Imprinting.

Biau G, Scornet E. 2016. A random forest guided tour. Test. 25(2):197-227. doi:10.1007/s11749-016-0481-7.

Bidlingmayer W, Evans D, Hansen C. 1985. Preliminary study of the effect of wind velocities and wind shadows upon suction trap catches of mosquitoes (Diptera: Culicidae). J Med Entomol. 22(3):295-302.

Bidlingmayer WL. 1964. The effect of moonlight on the flight activity of mosquitoes. Ecology. 45:87-94.

Bidlingmayer WL. 1967. A comparison of trapping methods for adult mosquitoes: species reponse and environmental influence. J Med Entomol. 4(2):200-220.

Bidlingmayer WL. 1971. Mosquito flight paths in relation to the environment. 1. Illumination levels, orientation, and resting areas. Ann Entomol Soc Am. 64(5):11211131. doi:10.1093/aesa/64.5.1121.

Bidlingmayer WL, Day JF, Evans DG. 1995. Effect of wind velocity on suction trap catches of some Florida mosquitoes. J Am Mosq Control Assoc. 11(3):295-301.

Bidlingmayer WL, Evans DG. 1987. The distribution of female mosquitoes about a flight barrier. J Am Mosq Control Assoc. 3:369-377.

Bidlingmayer WL, Hem DG. 1980. The range of visual attraction and the effect of competitive visual attractants upon mosquito (Diptera: Culicidae) flight. Bull Entomol Res. 70:321-342.

BioQuip Products. 2019. Standard New Jersey Light Trap. [accessed 2019 Oct 4]. www.bioquipinc.com/catalog/mosquitovector-equipment/standard-new-jersey-lighttrap/.

Blaschke T. 2010. Object based image analysis for remote sensing. ISPRS J Photogramm Remote Sens. 65(1):2-16. doi:10.1016/j.isprsjprs.2009.06.004.

Blaschke T, Burnett C, Pekkarinen A. 2004. New contextual approaches using image segmentation for object-based classification. In: De Meer F, de Jong S, editors. Remote Sensing Image Analysis: Including the spatial domain. Kluver Academic. p. 211-236.

Blaschke T, Hay GJ, Kelly M, Lang S, Hofmann P, Addink E, Queiroz Feitosa R, van der Meer F, van der Werff H, van Coillie F, et al. 2014. Geographic Object-Based Image Analysis - Towards a new paradigm. ISPRS J Photogramm Remote Sens. 87:180-191. 
doi:10.1016/j.isprsjprs.2013.09.014.

Bogojević MS, Merdić E, Bogdanović T. 2011. The flight distances of floodwater mosquitoes (Aedes vexans, Ochlerotatus sticticus and Ochlerotatus caspius) in Osijek, Eastern Croatia. Biologia (Bratisl). 66(4):678-683. doi:10.2478/s11756-011-0073-7.

Bowen MF. 1991. Host-Seeking Behavior in Mosquitoes. Annu Rev Entomol. 36:139158.

Bowles DE, Swaby JA. 2006. Field guide to venomous and medically important invertebrates affecting military operations: identification, biology, symptoms, treatment. Brooks City-Base, Texas: USAF Institue for Operational Health.

Bradter U, Kunin WE, Altringham JD, Thom TJ, Benton TG. 2013. Identifying appropriate spatial scales of predictors in species distribution models with the random forest algorithm. Methods Ecol Evol. 4(2):167-174. doi:10.1111/j.2041-210x.2012.00253.x.

Breiman L. 2001. Random Forests. Mach Learn. 45:5-32. doi:10.1201/978036781637711 .

Brenner J, Pocks DA, Arbogast T, Weaver K. 1998. Practical Use of Spatial Analysis in Pest Management. Am Entomol.(Summer):79-101.

Brenner RJ, Wargo MJ, Stains GS, Mulla MS. 1984. The dispersal of Culicoides mohave (Diptera: Ceratopogonidae) in the desert of southern California. Mosq News. 44:343350 .

Briegel H, Waltert A, Kuhn R. 2001. Reproductive physiology of Aedes (Aedimorphus) vexans (Diptera: Culicidae) in relation to flight potential. J Med Entomol. 38(4):557-565.

Brown H, Diuk-Wasser M, Andreadis T, Fish D. 2008. Remotely-sensed vegetation indices identify mosquito clusters of West Nile virus vectors in an urban landscape in the northeastern United States. Vector-Borne Zoonotic Dis. 8(2):197-206. doi:10.1089/vbz.2007.0154.

Bruce-Chwat L. 1985. Essential Malariology. 2nd ed. London: William Heineman.

Brust RA. 1980. Dispersal behavior of adult Aedes sticticus and Aedes vexans (Diptera: Culicidae) in Manitoba. Can Entomol. 112:31-42.

Brust RA. 1990. Oviposition behavior of natural populations of Culex tarsalis and Culex restuans (Diptera: Culicidae) in artificial pools. J Med Entomol. 27(2):248-255. doi:10.1093/jmedent/27.2.248.

Bunn JP, Balanay JAG, Richards SL. 2019. Evaluation of barrier sprays for controlling mosquitoes in Eastern North Carolina: can land cover and spatial analyses improve predictions of efficacy? J Am Mosq Control Assoc. 35(1):19-31. doi:10.2987/18-6762.1.

Burkett-Cadena ND, McClure CJW, Estep LK, Eubanks MD. 2013. Hosts or habitats: What drives the spatial distribution of mosquitoes? Ecosphere. 4(2):30. doi:10.1890/ES13-00009.1.

Caraballo H, King K. 2014. Emergency department management of mosquito-borne 
illness: malaria, dengue, and West Nile virus. Emerg Med Pract. 16(5):1-13.

Carnevale P, Frezil JL, Bosseno MF. 1978. Étude de l'agressivité d'Anopheles gambiae A en fonction de l'âge et du sexe des sujets humains. Bull World Health Organ. 56(1):147154.

Carpenter S, LaCasse W. 1955. Mosquitoes of North America (north of Mexico). Berkeley, CA: University of California Press.

CBC News. 2017 Jun 28. Winnipeg's mosquito count lowest in 40 years.

Cederlund GN, Okarma H. 1988. Home range and habitat use of adult female moose. J Wildl Manage. 52:336-343.

Chen S, Whiteman A, Li Ang, Rapp T, Delmelle E, Chen G, Brown CL, Robinson P, Coffman MJ, Janies D, et al. 2019. An operational machine learning approach to predict mosquito abundance based on socioeconomic and landscape patterns. Landsc Ecol. 2. doi:10.1007/s10980-019-00839-2.

Chiang GL, Loong KP, Chan ST, Eng KL, Yap HH. 1991. Capture-recapture studies with Anopheles maculatus Theobald (Diptera: Culicidae) the vector of malaria in peninsular Malaysia. Southeast Asian J Trop Med Public Health. 22(4):643-647.

Chuang T-W, Henebry GM, Kimball JS, Vanroekel-Patton DL, Hildreth MB, Wimberly MC. 2012. Satellite microwave remote sensing for environmental modeling of mosquito population dynamics. 125:147-156.

Chuang T-W, Hildreth MB, Vanroekel DL, Wimberly MC. 2011. Weather and land cover influences on mosquito populations in Sioux Falls, South Dakota. J Med Entomol. 48(3):669-679. doi:10.1603/ME10246.

Chuang T-W, Hockett CW, Kightlinger L, Wimberly MC. 2012. Landscape-level spatial patterns of West Nile virus risk in the northern Great Plains. Am J Trop Med Hyg. 86(4):724-731. doi:10.4269/ajtmh.2012.11-0515.

Chuang T-W, Wimberly MC. 2012. Remote sensing of climatic anomalies and West Nile virus incidence in the Northern Great Plains of the United States. PLoS One. 7(10). doi:10.1371/journal.pone.0046882.

City of Winnipeg. 2006. Annual report on mosquito surveillance and control in Winnipeg. Winnipeg, Manitoba.

City of Winnipeg. 2015. Annual report on mosquito surveillance and control in Winnipeg. Winnipeg, Manitoba.

City of Winnipeg. 2018. 2018 Orthographic Photography Tiles.

City of Winnipeg. 2019. Annual report on mosquito surveillance and control in Winnipeg. Winnipeg, Manitoba.

Clarke JL. 1937. New and significant experiences in mosquito control in the Desplaines Valley Mosquito Abatement District. Proc New Jersey Mosq Control Assoc. 24(112128). 
Clarke JL. 1943. Studies of the flight range of mosquitoes. J Econ Entomol. 36(1).

Clarke JLJ, Wray FC. 1967. Predicting influxes of Aedes vexans into urban areas. Mosq News. 27:156-163.

Clements ACA, Reid HL, Kelly GC, Hay SI. 2013. Further shrinking the malaria map: how can geospatial science help to achieve malaria elimination? Lancet Infect Dis. 13(8):709718.

Clements AN. 1963. The Physiology of Mosquitoes. New York: MacMillan.

Clymans R, Kerckvoorde V Van, Beliën T, Bylemans D, Clercq P De. 2020. Marking Drosophila suzukii (Diptera: Drosophilidae) with fluorescent dusts. Insects. 11(152).

Cohen J. 1960. A coefficient of agreement for nominal scales. Educ Psychol Meas. $\mathrm{XX}(1): 37-46$.

Congalton RG. 1991. A review of assessing the accuracy of classifications of remotely sensed data. Remote Sens Environ. 37(1):35-46. doi:10.1016/0034-4257(91)90048-B.

Congalton RG, Mead RA. 1983. A quantitative method to test for consistency and correctness in photointerpretation. Photogramm Eng Remote Sens. 49(1):69-74.

Conley AK, Fuller DO, Haddad N, Hassan AN, Gad AM, Beier JC. 2014. Modeling the distribution of the West Nile and Rift Valley Fever vector Culex pipiens in arid and semiarid regions of the Middle East and North Africa. Parasit Vectors. doi:10.1186/17563305-7-289.

Conway GR, Trpis M, McClelland GA. 1974. Population parameters of the mosquito Aedes aegypti (L.) estimated by mark-release-recapture in a suburban habitat in Tanzania. J Anim Ecol. 43(1):365-387.

Cook SP, Hain FP. 1992. The influence of self-marking with fluorescent powders on adult bark beetles (Coleoptera:Scolytidae). J Entomol Sci. 27(269-279).

Corbett A, Rosenheim JA. 1996. Quantifying movement of a minute parasitoid, Anagrus epos (Hymenoptera:Mymaridae), using fluorescent dust marking and recapture. Biol Control. 6:35-44.

Cortes C, Vapnik V. 1995. Support-vector networks. Mach Learn. 20:273-297. doi:10.1111/j.1747-0285.2009.00840.x.

Costantini C, Li SG, Della Torre A, Sagnon N, Coluzzi M, Taylor CE. 1996. Density, survival and dispersal of Anopheles gambiae complex mosquitoes in a West African Sudan savanna village. Med Vet Entomol. 10(3):203-219. doi:10.1111/j.13652915.1996.tb00733.x.

Coviella CE, Garcia JF, Jeske DR, Redak RA, Luck RF. 2006. Feasibility of tracking within-field movements of Homalodisca coagulata (Hemiptera: Cicadellidae) and estimating its densities using fluorescent dusts in mark-release-recapture experiments. J Econ Entomol. 99(1051-1057).

Craig MH, Snow RW, Le Suer D. 1999. A climate-based distribution model of malaria 
transmission in sub-Saharan Africa. Parasitol Today. 15(105-111).

Crocker W, Maute K, Webb C, French K. 2017. Mosquito assemblages associated with urban water bodies; implications for pest and public health threats. Landsc Urban Plan. 162:115-125.

Croux C, Dehon C. 2010. Influence functions of the Spearman and Kendall correlation measure. Stat Methods Appl. 19:497-515.

Crumpacker DW. 1974. The use of micronized fluorescent dusts to mark adult Drosophila pseudoobscura. Am Midl Nat. 91:118-129.

Curran PJ. 1988. The semivariogram in remote sensing: An introduction. Remote Sens Environ. 24(3):493-507.

Curtis C, Rawlings P. 1980. A preliminary study of dispersal and survival of Anopheles culicifacies in relation to the possibility of inhibiting the spread of insecticide resistance. Ecol Entomol. 5(1):11-17.

Cutler DR, Edwards TC, Beard KH, Cutler A, Hess KT, Gibson J, Lawler JJ. 2007. Random forests for classification in ecology. Ecology. 88(11):2783-2792. doi:10.1890/07-0539.1.

Dark SJ, Bram D. 2007. The modifiable areal unit problem (MAUP) in physical geography. Prog Phys Geogr. 31(5):471-479. doi:10.1177/0309133307083294.

Darsie RFJ, Ward RA. 2005. Identification and Geographical Distribution of the Mosquitoes of North America, North of Mexico. Gainesville, FL: American Mosquito Control Association.

De'Ath G, Fabricius KE. 2000. Classification and regression trees: A powerful yet simple technique for ecological data analysis. Ecology. 81(11):3178-3192. doi:10.1890/00129658(2000)081[3178:CARTAP]2.0.CO;2.

DeGroote JP, Sugumaran R, Brend SM, Tucker BJ, Bartholomay LC. 2008. Landscape, demographic, entomological, and climatic associations with human disease incidence of West Nile virus in the state of Iowa, USA. Int J Health Geogr. 7:1-16. doi:10.1186/1476072X-7-19.

Deichmeister JM, Telang A. 2011. Abundance of West Nile virus mosquito vectors in relation to climate and landscape variables. J Vector Ecol. 36(1):75-85.

DeMets S, Ziemann A, Manore C. 2020. Improving mosquito population predictions in the Greater Toronto Area using remote sensing imagery. Proc IEEE Southwest Symp Image Anal Interpret SSIAI.:To Appear.

Demets SA, Ziemann A, Manore C, Russell C. 2020. Too big, too small, or just right ? The influence of multispectral image size on mosquito population predictions in the Greater Toronto Area. 11392(May):1-8. doi:10.1117/12.2558128.

Denil M, Matheson D, De Freitas N. 2014. Narrowing the Gap: Random Forests In Theory and In Practice. Proc 31st Int Conf Mach Learn.(1998):665-673. 
Dennis B, Patil GP. 1988. Introduction. In: Lognormal Distributions: Theory and Applications. 1st ed. New York and Basel: Marcel Dekker, Inc. p. 303.

Diarra M, Fall M, Fall AG, Diop A, Lancelot R, Seck MT, Rakotoarivony I, Allène X, Bouyer J, Guis H. 2018. Spatial distribution modelling of Culicoides (Diptera: Ceratopogonidae) biting midges, potential vectors of African horse sickness and bluetongue viruses in Senegal. Parasit Vectors. 11(1):1-15. doi:10.1186/s13071-0182920-7.

Dickens BL, Brant HL. 2014. Effects of marking methods and fluorescent dusts on Aedes aegypti survival. Parasit Vectors. 7(1):1-9. doi:10.1186/1756-3305-7-65.

Diniz-Filho JAF, Bini LM, Hawkins BA. 2003. Spatial autocorrelation and red herrings in geographical ecology. Glob Ecol Biogeogr. 12(1):53-64. doi:10.1046/j.1466822X.2003.00322.x.

Diuk-Wasser MA, Brown HE, Andreadis TG, Fish D. 2006. Modeling the spatial distribution of mosquito vectors for West Nile virus in Connecticut, USA. Vector-Borne Zoonotic Dis. 6(3).

Dixon RD, Brust RA. 1972. Mosquitoes of Manitoba: III. Ecology of Larvae in the Winnipeg Area. Can Entomol. 104(7):961-968.

Dow RP, Reeves WC, Bellamy RE. 1965. Dispersal of female Culex tarsalis into a larvicided area. Am J Trop Med Hyg. 14(4):656-670.

Dowling Z, Ladeau SL, Armbruster P, Biehler D, Leisnham PT. 2013. Socioeconomic status affects mosquito (Diptera: Culicidae) larval habitat type availability and infestation level. J Med Entomol. 50(4):764-772.

Dyar HG. 1922. The mosquitoes of the United States. Proc US Natl Museum. 62(2447):1119.

Dye C, Davies CR, Lainson R. 1991. Communication among phlebotomine sandflies: a field study of domesticated Lutzomyia longipalpis populations in Amazonian Brazil. Anim Behav. 42:183-192.

Ecological Stratification Working Group. 1996. A National Ecological Framework For Canada. Ottawa/Hull, Ontario.

Edman J. 1971. Host-feeding patterns of Florida mosquitoes I. Aedes, Anopheles, Coquillettidia, Mansonia and Psorophora. J Med Entomol. 8(6):687-695.

Eid ANM, Olatubara CO, Ewemoje TA, El-Hennawy MT, Farouk H. 2020. Inland wetland time-series digital change detection based on SAVI and NDWI indecies: Wadi El-Rayan lakes, Egypt. Remote Sens Appl Soc Environ. 19(March):100347. doi:10.1016/j.rsase.2020.100347.

Eiras AE, Jepson PC. 1994. Responses of female Aedes aegypti (Diptera: Culicidae) to host odours and convection currents using an olfactometer bioassay. Bull Entomol Res. 84(2):207-211. 
Eisen L, Barker CM, Moore CG, Pape WJ, Winters AM, Cheronis N. 2010. Irrigated agriculture is an important risk factor for West Nile virus disease in the hyperendemic Larimer-Boulder-Weld area of North Central Colorado. J Med Entomol. 47(5):939-951.

Ellis JH. 1938. The Soils of Manitoba. Winnipeg, Manitoba.

Environment Canada. 2010. 1981-2010 Climate Normals \& Averages. Can Clim Norm.

Environment Canada. 2019. Hourly Data Report - Winnipeg A CS (Winnipeg James Armstrong Richardson International Airport). Hist Weather Data.

Epopa PS, Millogo AA, Collins CM, North A, Tripet F, Benedict MQ, Diabate A. 2017. The use of sequential mark-release-recapture experiments to estimate population size, survival and dispersal of male mosquitoes of the Anopheles gambiae complex in Bana, a west African humid savannah village. Parasit Vectors. 10(1):1-15. doi:10.1186/s13071017-2310-6.

Estallo EL, Sangermano F, Grech M, Ludueña-Almeida F, Frías-Cespedes M, Ainete M, Almirón W, Livdahl T. 2018. Modelling the distribution of the vector Aedes aegypti in a central Argentine city. Med Vet Entomol. 32(4):451-461. doi:10.1111/mve.12323.

Everingham YL, Lowe KH, Donald DA, Coomans DH, Markley J. 2007. Advanced satellite imagery to classify sugarcane crop characteristics. Agron Sustain Dev. 27(2):111-117.

Faiman R, Dao A, Yaro AS, Diallo M, Djibril S, Sanogo ZL, Ousmane Y, Sullivan M, Veru L, Krajacich BJ, et al. 2019. Marking mosquitoes in their natural larval sites using 2H-enriched water: A promising approach for tracking over extended temporal and spatial scales. Methods Ecol Evol. 10:1274-1285. doi:10.1111/2041-210X.13210.

Ferraguti M, Martínez-De La Puente J, Roiz D, Ruiz S, Soriguer R, Figuerola J. 2016. Effects of landscape anthropization on mosquito community composition and abundance. Sci Rep. 6.

Ferwerda C, Lathrop R. 2009. Characterizing the relationship between asian tiger mosquito abundance and habitat in urban New Jersey (MSc Thesis). Rutgers.

Field EN, Tokarz RE, Smith RC. 2019. Satellite imaging and long-term mosquito surveillance implicate the influence of rapid urbanization on Culex vector populations. Insects. 10(9):269. doi:10.3390/insects10090269.

Foody G. 2000. Estimation of sub-pixel land cover composition in the presence of untrained classes. Comput Geosci. 26(4):469-478.

Fouet C, Kamdem C. 2018. Integrated Mosquito Management : Is Precision Control a Luxury or Necessity? Trends Parasitol. xx:1-11. doi:10.1016/j.pt.2018.10.004.

Fryer JC, Meek CL. 1989. Further studies on marking an adult mosquito, Psorophora columbiae, in situ using fluorescent pigments. Southwest Entomol. 14:409-418.

Gao B-C. 1996. NDWI - A normalized difference water index for remote sensing of vegetation liquid water from space. Remote Sens Env. 7212(April):257-266. 
Garrett-Jones C. 1950. A dispersion of mosquitoes by wind. Nature. 165:285. doi:https://doi.org/10.1038/165285a0.

Geier M, Bosch OJ, Boeckh J. 1999. Influence of odour plume structure on upwind flight of mosquitoes towards hosts. J Exp Biol. 202:1639-48.

Giordano B, Turner K, Hunter F. 2018. Geospatial analysis and seasonal distribution of West Nile virus vectors (Diptera: Culicidae) in Southern Ontario, Canada. Int J Environ Res Public Health. 15(4):614. doi:10.3390/ijerph15040614.

Giordano B V, Bartlett SK, Falcon DA, Lucas RP, Tressler MJ, Campbell LP. 2020. Mosquito community composition, seasonal distributions, and trap bias in northeastern Florida. J Med Entomol.:1-9. doi:10.1093/jme/tjaa053.

Gleiser RM, Zalazar LP. 2010. Distribution of mosquitoes in relation to urban landscape characteristics. Bull Entomol Res. 100(2):153-158. doi:10.1017/S0007485309006919.

Glen S. "How to Find a Coefficient of Variation" From StatisticsHowTo.com: Elementary Statistics for the rest of us!

Goddard LB, Roth AE, Reisen WK, Scott TW. 2002. Vector competence of California mosquitoes for West Nile virus. Emerg Infect Dis. 8(12).

Government of Manitoba. 2002. The City of Winnipeg Charter Act.

Government of Manitoba. 2009. 1:20,000 Digital Topographic Mapping - Water.

Government of Manitoba. 2018. West Nile virus surveillance statistics.

Gubler DJ. 2002a. Epidemic dengue/dengue hemorrhagic fever as a public health, social and economic problem in the 21st century. Trends Microbiol. 10(2):100-103.

Gubler DJ. 2002b. The Global Emergence/Resurgence of Arboviral Diseases as Public Health Problems. Arch Med Res. 33:330-342.

Guerra CA, Reiner Jr RC, Perkins TA, Lindsay SW, Midega JT, Brady OJ, Barker CM, Reisen WK, Harrington LC, Takken W, et al. 2014a. A global assembly of adult female mosquito mark-release-recapture data to inform the control of mosquito-borne pathogens. Parasit Vectors. 7(276):1-15. doi:10.1186/1756-3305-7-276.

Guerra CA, Reiner Jr RC, Perkins TA, Lindsay SW, Midega JT, Brady OJ, Barker CM, Reisen WK, Harrington LC, Takken W, et al. 2014b. Additional file 3: Compressed folder containing the raw MMRR data. Parasit Vectors. 7(276).

de Guzman LI, Frake AM, Rinderer TE. 2012. Marking small hive beetles with thoracic notching: effects on longevity, flight ability and fecundity. Apidologie. 43:425-431.

Hagler JR, Jackson CG. 2001. Methods for marking insects: current techniques and future prospects. Annu Rev Entomol. 46:511-543.

Halasa YA, Shepard DM, Fonseca A, Farajollahi S, Healy R, Gaugler K, Bartlett-Healy D, Strickman DA, Clark GG. 2014. Quantifying the impact of mosquitoes on quality of life and enjoyment of yard and porch activities in New Jersey. PLoS One. 9:1-9. 
Hamer GL, Anderson TK, Donovan DJ, Brawn JD, Krebs BL, Gardner AM, Ruiz MO, Brown WM, Kitron UD, Newman CM, et al. 2014. Dispersal of adult Culex mosquitoes in an urban West Nile virus hotspot: A mark-capture study incorporating stable isotope enrichment of natural larval habitats. PLoS Negl Trop Dis. 8(3):6-12.

Han J, Kamber M, Pei J. 2011. Data Transformation by Normalization. In: Data mining: Concepts and Techniques. Elsevier Science. p. 113-114.

Harbach RE. 2013. Mosquito Taxonomic Inventory. Mosq Taxon Invent.

Harbach RE, Dallimore T, Briscoe AG, Culverwell CL, Vaux AGC, Medlock JM. 2017. Aedes nigrinus (Eckstein, 1918) (Diptera, Culicidae), a new country record for English, contrasted with Aedes sticticus (Meigen, 1938). Zookeys. 671:119-130.

Harrington LC, Scott TW, Lerdthusnee K, Coleman RC, Costero A, Clark GG, Jones JJ, Kitthawee S, Kittayapong P, Sithiprasasna R, et al. 2005. Dispersal of the dengue vector Aedes aegypti within and between rural communities. Am J Trop Med Hyg. 72(2):20920. doi:72/2/209 [pii].

Hay GJ, Marceau DJ, Dubé P, Bouchard A. 2001. A multiscale framework for landscape analysis: Object-specific analysis and upscaling. Landsc Ecol. 16:471-490.

Headlee TJ. 1945. The mosquitoes of New Jersey and their control. New Brunswick, NJ: Rutgers University Press.

Healy JM, Park B, White GS, Barker C. 2013. Development and validation of a fine-scale, spatio-temporal model to predict abundance of Culex mosquitoes in the Coachella Valley. In: Proceedings and Papers of the Mosquito and Vector Control Association of California. p. 64-67.

Hemme RR, Thomas CL, Chadee DD, Severson DW, Gubler DJ. 2010. Influence of urban landscapes on population dynamics in a short-distance migrant mosquito: evidence for the dengue vector Aedes aegypti. PLoS Negl Trop Dis. 4(3).

Hempelmann E, Krafts K. 2013. Bad air, amulets and mosquitoes: 2,000 years of changing perspectives on malaria. Malar J. 12(232).

Hengl T, Nussbaum M, Wright MN, Heuvelink GBM, Gräler B. 2018. Random forest as a generic framework for predictive modeling of spatial and spatio-temporal variables. PeerJ. 2018(8). doi:10.7717/peerj.5518.

Herold M, Gardner ME, Roberts DA. 2003. Spectral resolution requirements for mapping urban areas. IEEE Geosci Remote Sens. 41(9):1907-1919.

Hocking B. 1953. The intrinsic range and speed of flight of insects. Trans R Entomol Soc London. 104:223-345.

Honório N, da Costa Silva W, Leite PJ, Gonçalves JM, Lounibos LP, Lourenço-DeOliveira R. 2003. Dispersal of Aedes aegypti and Aedes albopictus (Diptera: Culicidae) in an urban endemic dengue area in the state of Rio de Janeiro, Brazil. Mem Inst Oswaldo Cruz. 98(2):191-198. 
Horsfall WR. 1954. A migration of Aedes vexans Meigen. J Econ Entomol. 47(3):544.

Horsfall WR. 1955. Mosquitoes: their bionomics and relation to disease. New York: Ronald Press.

Horsfall WR, Fowler HJ, Moretti L, Larsen J. 1973. Bionomics and embryology of the inland floodwater mosquito Aedes vexans. 1st ed. Urbana: University of Illinois Press.

Hossain MD, Chen D. 2019. Segmentation for Object-Based Image Analysis (OBIA): A review of algorithms and challenges from remote sensing perspective. ISPRS J Photogramm Remote Sens. 150(November 2018):115-134. doi:10.1016/j.isprsjprs.2019.02.009.

Hudson JE, Edman JD. 1978. Dispersal of blood-fed Culiseta inornata and Culiseta alaskaensis at Edmonton, Alberta. Mosq News. 38(1):87-89.

Ibañez-Justicia A, Cianci D. 2015. Modelling the spatial distribution of the nuisance mosquito species Anopheles plumbeus (Diptera: Culicidae) in the Netherlands. Parasit Vectors. 8(1):1-9. doi:10.1186/s13071-015-0865-7.

Izzah LN, Majid Z, Ariff MAM, Fook CK. 2016. Geospatial analysis of urban land use pattern analysis for hemorrhagic fever risk - a review. ISPRS - Photogramm Remote Sens Spat Inf Sci. XLII-4/W1(October):37-53.

Jackson BT, Paulson SL, Youngman RR, Sabra L, Hawkins B. 2005. Oviposition preferences of Culex restuans and Culex pipiens (Diptera: Culicidae) for selected infusions in oviposition traps and gravid traps. J Am Mosq Control Assoc. 21(4):360365.

James M, Harwood R. 1965. Herms's Medical Entomology. 1st, editor. New York: MacMillan Co.

Jaworski L, Jansen S, Pfitzner WP, Beck M, Becker N, Schmidt-Chanasit J, Kiel E, Lühken R. 2019. Comparative analysis of subsampling methods for large mosquito samples. Parasit Vectors. 12(1):354. doi:10.1186/s13071-019-3606-5.

Jenkins D, Hassett C. 1951. Dispersal and flight range of subarctic mosquitoes marked with radiophosphorus. Can J Zool. 29(3):178-187.

Jensen T, Washino RK. 1994. Comparison of recapture patterns of marked and released Aedes vexans and Ae. melanimon (Diptera: Culicidae) in the Sacramento Valley of California. J Med Entomol. 31(4):607-610. doi:10.1093/jmedent/31.4.607.

Johnson PH, Spitzauer V, Ritchie SA. 2012. Field sampling rate of BG-sentinel traps for Aedes aegypti (Diptera: Culicidae) in suburban Cairns, Australia. J Med Entomol. 49(1):29-34.

Juarez JG, Garcia-Luna S, Chaves LF, Carbajal E, Valdez E, Avila C, Tang W, Martin E, Barrera R, Hemme RR, et al. 2020. Dispersal of female and male Aedes aegypti from discarded container habitats using a stable isotope mark-capture study design in South Texas. Sci Rep. 10(1):1-12. doi:10.1038/s41598-020-63670-9. 
Kalluri S, Gilruth P, Rogers D, Szczur M. 2007. Surveillance of arthropod vector-borne infectious diseases using remote sensing techniques: A review. PLoS Pathog. 3(10):1361-1371. doi:10.1371/journal.ppat.0030116.

Kaplan G, Avdan U. 2017. Mapping and monitoring wetlands using Sentinel-2 satellite imagery. ISPRS Ann Photogramm Remote Sens Spat Inf Sci. 4(4/W4):271-277. doi:10.5194/isprs-annals-IV-4-W4-271-2017.

Karlin S. 1968. Rates of approach to homozygosity for finite stochastic model with variable population size. Am Nat. 102(927):443-455.

Keating J, Macintyre K, Mbogo C, Githeko A, Regens JL, Swalm C, Ndenga B, Steinberg LJ, Kibe L, Githure JI, et al. 2003. A geographic sampling strategy for studying relationships between human activity and malaria vectors in urban Africa. Am J Trop Med Hyg. 68(3):357-365. doi:10.4269/ajtmh.2003.68.357.

Kempala SDK. 1981. The ecology of Aedes (Stegomyia) africanus (Theobald) in a tropical forest in Uganda: mark-release-recapture studies on a female adult population. Int J Trop Insect Sci. 1(3):211-224.

Khan AA, Maibach HW, Strauss WG. 1968. The role of convection currents in mosquito attraction to human skin. Mosq News. 28(3):462-464.

Khatami R, Mountrakis G, Stehman S V. 2016. A meta-analysis of remote sensing research on supervised pixel-based land-cover image classification processes: General guidelines for practitioners and future research. Remote Sens Environ. 177:89-100. doi:10.1016/j.rse.2016.02.028.

Kitron U. 1998. Landscape ecology and epidemiology of vector-borne diseases: tools for spatial analysis. J Med Entomol. 35(4):435-445. doi:10.1093/jmedent/35.4.435.

Kitron U. 2000. Risk maps: Transmission and burden of vector-borne diseases. Parasitol Today. 16(8):324-325. doi:10.1016/S0169-4758(00)01708-7.

Kitron U, Kazmierczak JJ. 1997. Spatial analysis of the distribution of lyme disease in Wisconsin. Am J Epidemiol. 145(6):558-566. doi:10.1093/oxfordjournals.aje.a009145.

Kitron U, Otieno LH, Hungerford LL, Odulaja A, Brigham WU, Okello OO, Joselyn M, Mohamed-Ahmed MM, Cook E. 1996. Spatial analysis of the distribution of tsetse flies in the Lambwe Valley, Kenya, using Landsat TM satellite imagery and GIS. J Anim Ecol. 65(3):371-380. doi:10.2307/5883.

Kolivras KN. 2006. Mosquito habitat and dengue risk potential in Hawaii: A conceptual framework and GIS application. Prof Geogr. 58(2):139-154. doi:10.1111/j.14679272.2006.00521.x.

Kovats R, Campbell-Lendrum D, McMichael A, Woodward A, Cox J. 2001. Early effects of climate change: do they include changes in vector-borne disease? Philos Trans R Soc London Ser B-Biological Sci. 356(1411):1057-1068.

Krzywinski M, Altman N. 2013. Points of significance: Power and sample size. Nat Methods. 10(12):1139-1140. doi:10.1038/nmeth.2738. 
Kwon YS, Bae MJ, Chung N, Lee YR, Hwang S, Kim SA, Choi YJ, Park YS. 2015. Modeling occurrence of urban mosquitos based on land use types and meteorological factors in Korea. Int J Environ Res Public Health. 12(10):13131-13147. doi:10.3390/ijerph121013131.

LaBrecque GC, Bailey DL, Meifert DW, Weidhaas DE. 1975. Density estimates and daily mortality rate evaluations of stable fly (Stomoxys calcitrans (Diptera:Muscidae) populations in field cages. Can Entomol. 107(6):597-600.

Lacroix R, Delatte H, Hue T, Reiter P, Reiter AP. 2009. Dispersal and survival of male and female Aedes albopictus (Diptera: Culicidae) on Réunion Island. J Med Entomol. 46(465):1117-1124.

Lampman RL, Novak RJ. 1996. Attraction of Aedes albopictus adults to sod infusion. J Am Mosq Control Assoc. 12(1):119-124.

Landau KI, van Leeuwen WJD. 2012. Fine scale spatial urban land cover factors associated with adult mosquito abundance and risk in Tucson, Arizona. J Vector Ecol. 37(2):407418. doi:10.1111/j.1948-7134.2012.00245.x.

Landis R., Koch G. 1977. An application of hierarchical Kappa-type statistics in the assessment of majority agreement among multiple observers. Biometrics. 33(2):363-374.

Legendre P. 1993. Spatial Autocorrelation: Trouble or New Paradigm? Ecology. 74(6):1659-1673.

Lewis T, Taylor LR. 1967. Introduction to experimental ecology. London: Academic Press.

Li J, Deng T, Li H, Chen L, Mo J. 2010. Effects of water color and chemical compounds on the oviposition behavior of gravid Culex pipiens pallens females under laboratory conditions. J Agric Urban Entomol. 26(1):23-30. doi:10.3954/1523-5475-26.1.23.

Li M, Zang S, Zhang B, Li S, Wu C. 2014. A review of remote sensing image classification techniques: The role of Spatio-contextual information. Eur J Remote Sens. 47(1):389411. doi:10.5721/EuJRS20144723.

Li X, Wang Y. 2013. Applying various algorithms for species distribution modelling. Integr Zool. 8(2):124-135. doi:10.1111/1749-4877.12000.

Li Y, Kamara F, Zhou G, Puthiyakunnon S, Li C, Liu Y, Zhou Y, Yao L, Yan G, Chen X. 2014. Urbanization increases Aedes albopictus larval habitats and accelerates mosquito development and survivorship. PLoS Negl Trop Dis. 8(11):1-12.

Liaw A, Wiener M. 2002. Classification and Regression by randomForest. R News. 2(3):18-22.

Lillie TH, Kline DL, Hall DW. 1985. The dispersal of Culicoides mississippiensis (Diptera: Ceratopogonidae) in a salt marsh near Yankeetown, Florida. J Am Mosq Control Assoc. 1(4):463-467.

Lillie TH, Marquardt WC, Jones RH. 1981. The flight range of Culicoides variipennis. Can Entomol. 113:419-426. 
Liu Q, Liu X, Zhou G, Jiang J, Guo Y, Ren D, Zheng C, Wu H, Yang S, Liu J, et al. 2012. Dispersal range of Anopheles sinensis in Yongcheng City, China by mark-releaserecapture methods. PLoS One. 7(11):1-8.

Liu Q, Xie WJ, Xia JB. 2013. Using semivariogram and Moran's I techniques to evaluate spatial distribution of soil micronutrients. Commun Soil Sci Plant Anal. 44(7):11821192. doi:10.1080/00103624.2012.755999.

Loftin KM, Byford RL, Loftin MJ, Craig ME, Steiner RL. 1997. Host preference of mosquitoes in Bernalillo County, New Mexico. J Am Mosq Control Assoc. 13(1):71-75.

Looman J, Best KF. 1987. Budd's Flora of the Canadian Prairie Provinces. Regina, Saskatchewan, Canada: Research Branch Agriculture Canada.

Lorenz C, Charavalloti-Neto F, de Oliveira Lage M, Quintanilha JA, Parra MC, Dibo MR, Fávaro EA, Guirado M, Nogueria M. 2020. Remote sensing for risk mapping of Aedes aegypti infestations: Is this a practical task? Acta Trop. 205:105398.

Lothrop HD, Reisen WK. 1999. A geographical information system to manage mosquito and arbovirus surveillance and control data in the Coachella Valley of California. J Am Mosq Control Assoc. 15(3):299-307.

Macdonald W, Sebastian A, Helson B. 1968. A mark-release-recapture experiment with Culex pipiens fatigans in the village of Okpo, Burma. Ann Trop Med Parasitol. 62(2):200-209.

Madder DJ, MacDonald RS, Surgeoner GA. 1980. The use of oviposition activity to monitor populations of Culex pipiens and Culex restuans (Diptera: Culicidae). Can Entomol. 112(10):1013-1017.

Magnarelli LA. 1977. Host feeding patterns of Connecticut mosquitoes (Diptera: Culicidae). Am J Trop Med Hyg. 26(3):547-552.

Maktav D, Erbek FS, Jürgens C. 2005. Remote sensing of urban areas. Int J Remote Sens. 26(4):655-659. doi:10.1080/01431160512331316469.

Mallya S, Sander B, Roy-Gagnon M-H, Taljaard M, Jolly A, Kulkarni MA. 2018. Factors associated with human West Nile virus infection in Ontario: a generalized linear mixed modelling approach. BMC Infect Dis. 18(1):141. doi:10.1186/s12879-018-3052-6.

Manikandan S. 2011. Measures of central tendency: The mean. J Pharmacol Pharmacother. 2(2):140-142.

Marini F, Caputo B, Pombi M, Travaglio M, Montarsi F, Drago A, Rosà R, Manica M, della Torre A. 2019. Estimating spatio-temporal dynamics of Aedes albopictus dispersal to guide control interventions in case of exotic arboviruses in temperate regions. Sci Rep. 9(1):10281. doi:10.1038/s41598-019-46466-4.

McCrae AWR. 1984. Oviposition of African malaria vector mosquitoes II. Effects of site tone, water type and conspecific immatures on target selection by freshwater Anopheles gambiae Giles, sensu lato. Ann Trop Med Parasitol. 73(3):307-318. 
McFeeters SK. 1996. The use of the Normalized Difference Water Index (NDWI) in the delineation of open water features. Int $J$ Remote Sens. 17(7):1425-1432. doi:10.1080/01431169608948714.

McFeeters SK. 2013. Using the Normalized Difference Water Index (NDWI) within a geographic information system to detect swimming pools for mosquito abatement: A practical approach. Remote Sens. 5(7):3544-3561. doi:10.3390/rs5073544.

McMahon TJS, Galloway TD, Anderson R a. 2008. Tires as larval habitats for mosquitoes (Diptera: Culicidae) in southern Manitoba, Canada. J Vector Ecol. 33(1):198-204. doi:10.3376/1081-1710(2008)33[198:TALHFM]2.0.CO;2.

McNichol D. 2018. On Average, You're Using the Wrong Average: Geometric \& Harmonic Means in Data Analysis: When the Mean doesn't Mean what you think it Means. Medium.

Medeiros MCI, Boothe EC, Roark EB, Hamer GL. 2017. Dispersal of male and female Culex quinquefasciatus and Aedes albopictus mosquitoes using stable isotope enrichment. PLoS Negl Trop Dis. 11(1):1-24. doi:10.1371/journal.pntd.0005347.

Meek CL, Broussard BB, Andis MD. 1988. Marking adult mosquitoes using fluorescent pigments in dispersal studies. Bull Soc Vector Ecol. 13:319-322.

Mi C, Huettmann F, Guo Y, Han X, Wen L. 2017. Why choose Random Forest to predict rare species distribution with few samples in large undersampled areas? Three Asian crane species models provide supporting evidence. PeerJ. doi:10.7717/peerj.2849.

Milby MM, Reeves WC. 1989. Comparison of New Jersey light-traps and $\mathrm{CO}_{2}$-baited traps in urban and rural areas. Proc Calif Mosq Vector Control Assoc. 57:73-79.

Moffitt HR, Albano DJ. 1972. Codling months: fluorescent powders as markers. Environ Entomol. 1(6):750-753.

Molaei G, Andreadis TG, Armstrong PM, Diuk-Wasser M. 2008. Host-feeding patterns of potential mosquito vectors in Connecticut, USA: Molecular analysis of bloodmeals from 23 species of Aedes, Anopheles, Culex, Coquillettidia, Psorophora, and Uranotaenia. J Med Entomol. 45(6):1143-1151.

Moncayo AC, Edman JD, Finn JT. 2000. Application of geographic information technology in determining risk of Eastern Equine Encephalomyelitis virus transmission. J Am Mosq Control Assoc. 16(1):28-35.

Moore CG, McLean RG, Mitchell CJ, Nasci RS, Tsai TF, Calisher CH, Marfin AA, Moore PS, Gubler DJ. 1993. Guidelines for arbovirus surveillance programs in the United States. Fort Collins, Colorado.

Morris CD, Larson VL, Lounibos LP. 1991. Measuring mosquito dispersal for control programs. J Am Mosq Control Assoc. 7(4):608-615.

Mudele O, Bayer FM, Zanandrez L, Eiras AE, Gamba P. 2019. Modeling the temporal population distribution of Ae. aegypti mosquito using Big Earth observation data. IEEE Access. 8:14182-14194. doi:10.1109/ACCESS.2020.2966080. 
Muir LE, Kay BH. 1998. Aedes aegypti survival and dispersal estimated by mark-releaserecapture in Northern Australia. Am J Trop Med Hyg. 58(3):277-282.

Mulhern TD. 1934. A new development in mosquito traps. Proc New Jersey Mosq Exterm Assoc. 20:137.

Mullen GR. 1971. Diurnal resting sites of mosquitoes based on sweeping collections in central New York. Proc New Jersey Mosq Exterm Assoc. 58:185-188.

Muñoz SR, Bangdiwala SI. 1997. Interpretation of Kappa and B statistics measures of agreement. J Appl Stat. 24(1):105-112. doi:10.1080/02664769723918.

Murdock CC, Evans M V., McClanahan TD, Miazgowicz KL, Tesla B. 2017. Fine-scale variation in microclimate across an urban landscape shapes variation in mosquito population dynamics and the potential of Aedes albopictus to transmit arboviral disease. PLoS Negl Trop Dis. 11(5):1-23. doi:10.1371/journal.pntd.0005640.

Napa County Mosquito Abatement District. Supplemental Information: Mosquitoes. [accessed $2020 \quad$ Mar 11]. https://napamosquito.org/vectors-andpests/mosquitoes/supplemental-information-mosquitoes/.

Naranjo SE. 1990. Influence of two mass-marking techniques on survival and flight behavior of Diabrotica virgifera (Coleoptera:Chrysomelidae). J Econ Entomol. 83:13601364.

Natural Resources Canada. 2015. Land Cover \& Land Use. Gov Canada. [accessed 2020 Dec 12]. https://www.nrcan.gc.ca/maps-tools-and-publications/satellite-imagery-andair-photos/remote-sensing-tutorials/land-cover-land-use/9373.

Nayar JK. 1985. Bionomics and physiology of Aedes taeniorhynchus and Aedes sollicitans, the salt marsh mosquitoes of Florida.

Nayar JK, Sauerman DM. 1969. Flight behavior and flight polymorphism in the mosquito Aedes taeniorhynchus. Entomol Exp Appl. 12(365-375).

Nayar JK, Sauerman DM. 1973. A comparative study of growth and development in Florida mosquitoes. Part 4. Effects of temporary crowding during larvae stages on female flight activity patterns. J Med Entomol. 10:37-42.

Nelson RL, Milby MM. 1980. Effects of fluorescent marker dusts on Culex tarsalis, a factor in mark-release-recapture studies. In: Proceedings and Papers of the Mosquito and Vector Control Association of California, 48. p. 66-68.

Nelson RL, Milby MM, Reeves WC, Fine PEM. 1978. Estimates of survival, population size, and emergence of Culex tarsalis at an isolated site. Ann Entomol Soc Am. 71(5):801-808.

Nielsen CF, Armijos MV, Wheeler S, Carpenter TE, Boyce WM, Kelley K, Brown D, Scott TW, Reisen WK. 2008. Risk factors associated with human infection during the 2006 West Nile virus outbreak in Davis, a residential community in Northern California. 78(1):53-62. 
Nolan MS, Zangeneh A, Khuwaja SA, Martinez D, Rossmann SN, Cardenas V, Murray KO. 2012. Proximity of residence to bodies of water and risk for West Nile virus infection: A case-control study in Houston, Texas. J Biomed Biotechnol.:6. doi:10.1155/2012/159578.

O’Malley CM. 1990. Aedes vexans (Meigen): An old foe. Proc New Jersey Mosq Control Assoc. 77:90-95.

Oaks JSC, Mitchell VS, Pearson GW, Carpenter CCJ (Eds. . 1991. Malaria; Obstacles and Opportunities. Oaks JSC, Mitchell VS, Pearson GW, Carpenter CCJ (Eds. ., editors.

Olanga EA, Okal MN, Mbadi PA, Kokwaro ED, Mukabana WR. 2010. Attraction of Anopheles gambiae to odour baits augmented with heat and moisture. Malar J. 9(6):110 .

Ong J, Liu X, Rajarethinam J, Kok SY, Liang S, Tang CS, Cook AR, Ng LC, Yap G. 2018. Mapping dengue risk in Singapore using Random Forest. PLoS Negl Trop Dis. 12(6):112. doi:10.1371/journal.pntd.0006587.

Ostfeld RS, Glass GE, Keesing F. 2005. Spatial epidemiology: An emerging (or reemerging) discipline. Trends Ecol Evol. 20(6 SPEC. ISS.):328-336. doi:10.1016/j.tree.2005.03.009.

Pal NR, Pal SK. 1993. A review of image segmentation techniques. Pattern Recognit. 26:1277-1294.

Palaniyandi M. 2012. The role of remote sensing and GIS for spatial prediction of vectorborne diseases transmission: A systematic review. J Vector Borne Dis. 49:197-204.

Peterson DG, Brown AWA. 1951. Studies of the responses of the female Aedes mosquito. Part III. The response of Aedes aegypti (L.) to a warm body and its radiation. Bull Entomol Res. 42(3):535-541.

Piedrahita S, Altamiranda-Saavedra M, Correa MM. 2020. Spatial fine-resolution model of malaria risk for the Colombian Pacific region. Trop Med Int Heal. 10. doi:10.1111/tmi.13443.

Platt RB, Collins CL, Witherspoon JP. 1957. Reactions of Anopheles quadrimaculatus Say to moisture temperature and light. Ecol Monogr. 21:303-324.

Poirier LM, Berry KE. 2011. New distribution information for Coquillettidia perturbans (Walker) (Diptera, Culicidae) in northern British Columbia, Canada. J Vector Ecol. 36(2):461-463. doi:10.1111/j.1948-7134.2011.00190.x.

Poland TM, Haack RA, Petrice TR. 2000. Dispersal of Tomicus piniperda (Coleoptera : Scolytidae) from operational and simulated mill yards. Can Entomol. 132:853-866.

Porphyre T, Bicout DJ, Sabatier P. 2005. Modelling the abundance of mosquito vectors versus flooding dynamics. Ecol Modell. 183(2-3):173-181. doi:10.1016/j.ecolmodel.2004.06.044.

Price RD, Olson TA, Rueger ME, Schlottman LL. 1960. A survey of potential 
overwintering sites of Culex tarsalis Coquillett in Minnesota. Mosq News. 20(3):306311.

Puletti N, Perria R, Storchi P. 2014. Undersupervised classification of very high remotely sensed images for grapevine rows detection. Eur J Remote Sens. 47:45-54.

Qiao H, Sobéron J, Peterson AT. 2015. No silver bullets in correlative ecological niche modelling: insights from testing among many potential algorithms for niche estimation. Methods Ecol Evol. 6(10).

R Core Team. 2019. R: A language and environment for statistical computing.

Rees DM. 1943. The Mosquitoes of Utah. Bull Univ Utah.

Rees DM, Nielsen LT. 1947. On the biology and control of Aedes dorsalis (Meigen) in Utah. Proc New Jersey Mosq Exterm Assoc. 34:160-165.

Reeves WC, Brookman B, Hooman WM. 1948. Studies on the flight range of certain Culex mosquitoes, using a fluorescent dye-marker, with notes on Culiseta and Anopheles. Mosq News. 8(2):61-69.

Reid TG, Reid ML. 2008. Fluorescent powder marking reduces condition but not survivorship in adult mountain pine beetles. Can Entomol. 140(5):582-588. doi:10.4039/N08-035.

Reinert JF. 2000. New classification for the composite genus Aedes (Diptera: Culicidae: Aedini), elevation of subgenus Ochlerotatus to generic rank, reclassification of the other subgenera, and notes on certain subgenera and spesies. J Am Mosq Control Assoc. 16(3):175-188.

Reinert JF, Harbach RE, Kitching IJ. 2008. Phylogeny and classification of Ochlerotatus and allied taxa (Diptera: Culicidae: Aedini) based on morphological data from all life stages. Zool J Linn Soc. 153:29-114.

Reinert JF, Harbach RE, Kitching IJ. 2009. Phylogeny and classification of tribe Aedini (Diptera: Culicidae). Zool J Linn Soc. 157(4):700-794. doi:10.1111/j.10963642.2009.00570.x.

Reinert JF REH, Kitching IJ. 2004. Phylogeny and classification of Aedini (Diptera: Culicidae) based on morphological data from all life stages. Zool J Linn Soc. 142:289368.

Reinert JF REH, Kitching IJ. 2006. Phylogeny and classification of Finlaya and allied taxa (Diptera: Culicidae: Aedini) based on morphological data from all life stages. Zool J Linn Soc. 148:1-101.

Reinert W. 1989. The New Jersey Light Trap: An Old Standard for Most Mosquito Control Programs. In: Proceedings of the Seventy-Sixth Annual Meeting of the New Jersey Mosquito Control Association, Inc. p. 17-25.

Reisen WK. 2010. Landscape Epidemiology of Vector-Borne Diseases. Annu Rev Entomol. 55:461-83. doi:10.1146/annurev-ento-112408-085419. 
Reisen WK. 2016. Update on Journal Policy of Aedine Mosquito Genera and Subgenera. J Med Entomol. 53(2):249-249. doi:10.1093/jme/tjv169.

Reisen WK, Asman SM, Milby MM, Bock ME, Stoddard PJ, Meyer RP, Reeves WC. 1981. Attempted suppression of a semi-isolated population of Culex tarsalis by release of irradiated males. Mosq News. 41:736-744.

Reisen WK, Eldridge BF, Scott TW, Gutierrez A, Takahashi R, Lorenzen K, DeBenedictis J, Boyce K, Swartzell R. 2002. Comparison of dry ice-baited Centers for Disease Control and New Jersey light traps for measuring mosquito abundance in California. J Am Mosq Control Assoc. 18:158-163.

Reisen WK, Knop NF, Peloquin JJ. 1985. Swarming and mating behavior of laboratory and field strains of Culex tarsalis (Diptera: Culicidae). Ann Entomol Soc Am. 78(5):667673.

Reisen WK, Lothrop HD. 1995. Population ecology and dispersal of Culex tarsalis in the Coachella valley of California. J Med Entomol. 32(4):490-502.

Reisen WK, Lothrop HD. 1999. Effects of sampling design on the estimation of adult mosquito abundance. J Am Mosq Control Assoc. 15(2):105-114.

Reisen WK, Lothrop HD, Lothrop B. 2003. Factors influencing the outcome of markrelease-recapture studies with Culex tarsalis (Diptera: Culicidae). J Med Entomol. 40(6):820-829. doi:10.1603/0022-2585-40.6.820.

Reisen WK, Mahmood F, Parveen T. 1979. Anopheles subpictus Grassi: Observation on survivorshop and population size using mark-release-recapture and dissection methods. Res Popul Ecol (Kyoto). 21(1):12-29.

Reisen WK, Mahmood F, Parveen T. 1980. Anopheles culicifacies Giles: a releaserecapture experiment with cohorts of known age with implications for malaria epidemiology and genetical control for Pakistan. Trans R Soc Trop Med Hyg. 74(3):307317.

Reisen WK, Meyer R., Tempelis CH, Spoehel JJ. 1990. Mosquito abundance and bionomics in residential communities in Orange and Los Angeles Counties, California. J Med Entomol. 27:356-367.

Reisen WK, Milby MM, Meyer RP. 1992. Population dynamics of adult Culex mosquitoes (Diptera: Culicidae) along the Kern River, Kern County, California, in 1990. J Med Entomol. 29(3):531-543. doi:10.1093/jmedent/29.3.531.

Reisen WK, Pfuntner AR, Milby MM, Tempelis CH, Presser SB. 1990. Mosquito bionomics and the lack of arbovirus activity in the Chino area of San Bernadino County, California. J Med Entomol. 27:811-818.

Reiter ME, LaPointe DA. 2007. Landscape factors influencing the spatial distribution and abundance of mosquito vector Culex quinquefasciatus (Diptera: Culicidae) in a mixed residential -agricultural community in Hawai'i. J Med Entomol. 44(5):861-868. doi:10.1603/0022-2585(2007). 
Reiter P. 2000. From Shakespeare to Defoe: malaria in England in the Little Ice Age. Emerg Infect Dis. 6(1):1-11.

Reiter P. 2001. Climate change and mosquito-borne disease. Environ Health Perspect. doi: $10.2307 / 3434853$.

Reiter P, Amador MA, Anderson RA, Clark GG. 1995. Short report: Dispersal of Aedes aegypti in an urban area after blood feeding as demonstrated by rubidium-marked eggs. Am J Trop Med Hyg. 52(2):177-179. doi:10.4269/ajtmh.1995.52.177.

Rey JR, Nishimura N, Wagner B, Braks MAH, O'Connell SM, Lounibos LP. 2006. Habitat segregation of mosquito arbovirus vectors in South Florida. J Med Entomol. 43(6):11341141. doi:10.1093/jmedent/43.6.1134.

Rhodes DJ, Hayes JL, Steiner C. 1998. Retention of external and internal markers by southern pine beetles (Coleoptera: Scolytidae) during gallery construction. J Entomol Sci. $33: 221-232$.

Richards SL, Ponnusamy L, Unnasch TR, Hassan HK, Apperson CS. 2006. Host-feeding patterns of Aedes albopictus (Diptera: Culicidae) in relation to availability of human and domestic animals in suburban landscapes of central North Carolina. J Med Entomol. 43(3):543-551.

Ridd MK. 1995. Exploring a V-I-S (Vegetation-impervious surface-soil) model for urban ecosystem analysis through remote sensing: Comparative anatomy for citiest. Int $\mathbf{J}$ Remote Sens. 16(12):2165-2185. doi:10.1080/01431169508954549.

Roche JP. 2002. Print media coverage of risk-risk tradeoffs associated with West Nile encephalitis and pesticide spraying. J Urban Heal. 79(4):482-490. doi:10.1093/jurban/79.4.482.

Rojas-Araya D, Alto BW, Burkett-cadena N, Cummings DAT. 2019. Detection of fluorescent powders and their effect on survival and recapture of Aedes aegypti (Diptera : Culicidae). J Med Entomol. X(X):1-7. doi:10.1093/jme/tjz142.

Rojas-Araya D, Alto BW, Burkett-Cadena ND, Cummings DAT. 2020. Impacts of fluorescent powders on survival of different age cohorts, blood-feeding success, and tethered flight speed of Aedes aegypti (Diptera: Culicidae) females. Acta Trop. In Press(Journal Pre-proof).

Rokach L, Maimon O. 2015. Data Mining with Decision Trees.

Rowe JS. 1956. Vegetation of the Southern Boreal Forest of Manitoba and Saskatchewan.

Rowley WA. 2015. Laboratory Flight Ability of the Mosquito, Culex tarsalis Coq. J Med Entomol. 7(6):713-716. doi:10.1093/jmedent/7.6.713.

Rueda LM. 2008. Global diversity of mosquitoes (Insecta: Diptera: Culicidae) in freshwater. Hydrobiologia. 595(1):477-487. doi:10.1007/s10750-007-9037-x.

Ruiz MO, Tedesco C, McTighe TJ, Austin C, Uriel K. 2004. Environmental and social determinants of human risk during a West Nile virus outbreak in the greater Chicago area, 
2002. Int J Health Geogr. 3:1-11. doi:10.1186/1476-072X-3-8.

Russell RC, Webb CE, Williams CR, Ritchie SA. 2005. Mark-release-recapture study to measure dispersal of the mosquito Aedes aegypti in Cairns, Queensland, Australia. Med Vet Entomol. 19(4):451-457. doi:10.1111/j.1365-2915.2005.00589.x.

Russo RJ. 1977. Substrate moisture effects upon oviposition in Aedes vexans. Mosq News. 37(712-717).

Ryan PA, Lyons SA, Alsemgeest D, Thomas P, Kay BH. 2004. Spatial statistical analysis of adult mosquito (Diptera: Culicidae) counts: an example using light trap data, in Redland Shire, Southeastern Queensland, Australia. J Med Entomol. 41(6):1143-1156.

Sallam MF, Fizer C, Pilant AN, Whung PY. 2017. Systematic review: Land cover, meteorological, and socioeconomic determinants of Aedes mosquito habitat for risk mapping. Int J Environ Res Public Health. 14(10). doi:10.3390/ijerph14101230.

Sallam MF, Michaels SR, Riegel C, Pereira RM, Zipperer W, Graeme Lockaby B, Koehler PG. 2017. Spatio-temporal distribution of vector-host contact (VHC) ratios and ecological niche modeling of the West Nile virus mosquito vector, Culex quinquefasciatus, in the City of New Orleans, LA, USA. Int J Environ Res Public Health. 14(8):1-20. doi:10.3390/ijerph14080892.

Sarnekis K. 2002. Mosquitoes in Constructed Wetlands. Adelaide, Australia.

Savage H., Strickman D. 2004. The genus and subgenus categories within Culicidae and placement of Ochlerotatus as a subgenus of Aedes. J Am Mosq Control Assoc. 20(2):208-214.

Schäfer M, Lundstrom L, Pfeffer JO, Lundkvist M, Landin EJ. 2004. Biological diversity versis risk for mosquito nuisance and disease transmission in constructed wetlands in southern Sweden. Med Vet Entomol. 18:256-267.

Schäfer M, Storch V, Kaiser A, Beck M, Becker N. 1997. Dispersal behavior of adult snow melt mosquitoes in the Upper Rhine Valley, Germany. J Vector Ecol. 22:1-5.

Schellenberg M, Canada A, Centre D, Current S. 2016. Management of Canadian Prairie. (January 2010).

Sciarretta A, Trematerra P. 2014. Geostatistical tools for the study of insect spatial distribution: Practical implications in the integrated management of orchard and vineyard pests. Plant Prot Sci. 50(2):97-110. doi:10.17221/40/2013-pps.

Sellers PJ. 1985. Canopy reflectance, photosynthesis and transpiration. Int J Remote Sens. 6(8):1335-1372. doi:10.1080/01431168508948283.

Sellers RF, Maarouf AR. 1988. Impact of climate on western equine encephalitis in Manitoba, Minnesota and North Dakota, 1980-1983. Epidemiol Infect. 101(3):511-535. doi:10.1017/S095026880002940X.

Sérandour J, Willison J, Thuiller W, Ravanel P, Lempérière G, Raveton M. 2010. Environmental drivers for Coquillettidia mosquito habitat selection: A method to 
highlight key field factors. Hydrobiologia. 652(1):377-388. doi:10.1007/s10750-0100372-y.

Service MW. 1976. Mosquito Ecology, Field Sampling Methods. Halsted Press.

Service MW. 1980. Effects of wind on the behaviour and distribution of mosquitoes and blackflies. Int J Biometeorol. 24(4):347-353.

Service MW. 1993. Mosquito Ecology: Field Sampling Methods. 2nd ed. Elsevier Applied Science.

Service MW. 1997. Mosquito (Diptera : Culicidae) Dispersal — The Long and Short of It. J Med Entomol. 34(6):579-588.

Service MW. 2008. Medical entomology for students, fourth edition.

Shaman J, Day JF. 2005. Achieving operational hydrologic monitoring of mosquitoborne disease. Emerg Infect Dis. 11(9):1343-1350. doi:10.3201/eid1109.050340.

Shaman J, Stieglitz M, Stark C, Le Blancq S, Cane M. 2002. Using a dynamic hydrology model to predict mosquito abundances in flood and swamp water. Emerg Infect Dis @BULLET. 8(1).

Shaw IGR, Jones JP, Butterworth MK. 2013. The mosquito's umwelt, or one monster's standpoint ontology. Geoforum. 48:260-267.

Sheather S. 2009. A Modern Approach to Regression with R.

Shemanchuk JA. 1965. On the hibernation of Culex tarsalis Coquillett, Culiseta inornata Williston and Anopheles earlei Vargas, (Diptera: Culicidae) in Alberta. Mosq News. 456-462(25):4.

Shemanchuk JA. 1969. Epidemiology of western encephalitis in Alberta: response of natural populations of mosquitoes to avian host. J Med Entomol. 6(3):269-275.

Silver JB. 2008. Mosquito Ecology-Field Sampling Methods 3rd Edition.

Singh A. 1989. Review Article: Digital change detection techniques using remotely-sensed data. Int J Remote Sens. 10(6):989-1003. doi:10.1080/01431168908903939.

Smith A. 1956. The attractiveness of an adult and child to A. gambiae. East Afr Med J. 33(10):409-410.

Snow WF. 1976. The direction of flight of mosquitoes (Diptera, Culicidae) near the ground in West African savanna in relation to wind direction, in the presence and absence of bait. Bull Entomol Res. 65(04):555.

Stage HH, Gjullin CM, Yates WW. 1937. Flight range and longevity of floodwater mosquitoes in the lower Columbia River Valley. J Econ Entomol. 30:940-945.

Staples JE, Shankar MB, Sejvar JJ, Meltzer MI, Fischer M. 2014. Initial and long-term costs of patients hospitalized with West Nile virus disease. Am J Trop Med Hyg. 90:402409. 
Statistics Canada. 2016. The changing landscape of Canadian metropolitan areas.

Stefanov WL, Ramsey MS, Christensen PR. 2001. Monitoring urban land cover change : An expert system approach to land cover classification of semiarid to arid urban centers. Remote Sens Envi. 77:173-185.

Strobl C, Boulesteix AL, Zeileis A, Hothorn T. 2007. Bias in random forest variable importance measures: Illustrations, sources and a solution. BMC Bioinformatics. 8(25):21. doi:10.1186/1471-2105-8-25.

Su T, Webb JP, Meyer RP, Mulla MS. 2003. Spatial and temporal distribution of mosquitoes in underground storm drain systems in Orange County, California. J Vector Ecol. 28(1):79-89.

Suggitt AJ, Gillingham PK, Hill JK, Huntley B, Kunin WE, Roy DB, Thomas CD. 2010. Habitat microclimates drive fine-scale variation in extreme temperatures. Oikos. 120:18.

Tabachnick BG, Fidell FS. 2013. Using Multivariate Statistics. 6th ed. Pearson.

Takken W. 1991. The role of olfaction in host-seeking of mosquitoes: a review. Insect Sci Its Appl. 12(287-295).

Takken W, Charlwood JD, Billingsley PF, Gort G. 1998. Dispersal and survival of Anopheles funestus s.l. (Diptera:Culicidae) during the rainy season in southeast Tanzania. Bull Entomol Res. 88:561-566.

Takken W, Knols BGJ. 1999. Odor-Mediated Behavior of Afrotropical Malaria Mosquitoes. Annu Rev Entomol. 44(1):131-157. doi:10.1146/annurev.ento.44.1.131.

Takken W, Verhulst NO. 2013. Host Preferences of Blood-Feeding Mosquitoes. Annu Rev Entomol. 58:433-53. doi:10.1146/annurev-ento-120811-153618.

Tempelis CH, Francy DB, Hayes RO, Lofy MF. 1967. Variations in feeding patterns of seven Culicine mosquitoes on vertebrate hosts in Weld and Larimer Counties, Colorado. Am J Trop Med Hyg. 16(1):111-119.

Thanh Noi P, Kappas M. 2017. Comparison of Random Forest, k-Nearest Neighbor, and Support Vector Machine classifiers for land cover classification Uusing Sentinel-2 imagery. Sensors. 18(1). doi:10.3390/s18010018.

Therneau T, Atkinson E. 2013. An introduction to Recursive Partitioning Using the RPART Routines.

Thiemann T, Nelms B, Reisen WK. 2011. Bloodmeal host congregation and landscape structure impact the estimation of female mosquito (Diptera: Culicidae) abundance using dry ice-baited traps. J Med Entomol. 48(3):513-517.

Tjaden NB, Caminade C, Beierkuhnlein C, Thomas SM. 2018. Mosquito-Borne Diseases: Advances in Modelling Climate-Change Impacts. Trends Parasitol. 34(3):227-245. doi:10.1016/j.pt.2017.11.006.

Tobler WR. 1970. A Computer Movie Simulating Urban Growth in the Detroit Region. 
Econ Geogr. 46(Supplement: Proceedings. International Geographical Union. Commission on Quantitative Methods):234-240.

Tokarz R, Novak RJ. 2018. Spatial - temporal distribution of Anopheles larval habitats in Uganda using GIS / remote sensing technologies. Malar J.:1-14. doi:10.1186/s12936018-2567-z.

Tomerini DM, Dale PE, Sipe N. 2011. Does mosquito control have an effect on mosquitoborne disease? The case of Ross River virus disease and mosquito management in Queensland, Australia. J Am Mosq Control Assoc. 27(1):39-44. doi:10.2987/10-6038.1.

Trawinski PR, Mackay DS. 2010. Identification of environmental covariates of West Nile virus vector mosquito population abundance. Vector-Borne Zoonotic Dis. 10(5):515526. doi:10.1089/vbz.2008.0063.

Trewin BJ, Pagendam DE, Zalucki MP, Darbro JM, Devine GJ, Jansen CC, Schellhorn NA. 2019. Urban landscape features influence the movement and distribution of the Australian container-inhabiting mosquito vectors Aedes aegypti (Diptera: Culicidae) and Aedes notoscriptus (Diptera: Culicidae). J Med Entomol. $\mathrm{xx}(\mathrm{X}): 1-11$. doi:10.1093/jme/tjz187.

Tsuda Y, Kamezaki H. 2014. Mark-release-recapture study on movement of mosquitoes : individual marking method and short-term study of Aedes albopictus and Armigeres subalbatus in residential area on Ishigaki island, Japan. Med Entomol Zool. 65(2):61-66. doi:10.7601/mez.65.61.

Tufto J, Andersen R, Linnell J. 1996. Habitat use and ecological correlates of home range size in a small cervid: the roe deer. J Anim Ecol. 65:715-724.

Tun-Lin W, Burkot TR, Kay BH. 2000. Effects of temperature and larval diet on development rates and survival of the dengue vector Aedes aegypti in north Queensland, Australia. Med Vet Entomol. 14(1):31-37.

Tun-Lin W, Kay BH, Barnes A. 1995. The Premise Condition Index: A tool for streamlining surveys of Aedes aegypti. Am J Trop Med Hyg. 53(6):591-594.

Turell MJ, Dohm DJ, Sardelis MR, O’guinn ML, Andreadis TG, Blow JA. 2005. An update on the potential of North American mosquitoes (Diptera: Culicidae) to transmit West Nile virus. J Med Entomol. 42(1):57-62. doi:10.1093/jmedent/42.1.57.

Turner MG. 1989. Landscape ecology: the effect of pattern on process. Annu Rev Ecol Syst. 20:171-197. doi:10.1146/annurev.es.20.110189.001131.

United States Department of Agriculture. 1996. Riparian Area Environmental Uniqueness, Functions and Values. RCA Issue $\mathrm{Br} \# 11$.

US Environmental Protection Agency. 2017. Controlling Adult Mosquitoes. Environ Top.

Utz JT, Apperson CS, MacCormack JN, Salyers M, Dietz EJ, McPherson JT. 2018. Economic and social impacts of La Crosse encephalitis in western North Carolina. Am J Trop Med Hyg. 69(5):509-518. doi:10.4269/ajtmh.2003.69.509. 
Uusitalo R, Siljander M, Culverwell CL, Mutai NC, Forbes KM, Vapalahti O, Pellikka PKE. 2019. Predictive mapping of mosquito distribution based on environmental and anthropogenic factors in Taita Hills, Kenya. Int J Appl Earth Obs Geoinf. 76(November 2018):84-92. doi:10.1016/j.jag.2018.11.004.

Vanwambeke SO, Lambin EF, Eichhorn MP, Flasse SP, Harbach RE, Oskam L, Somboon P, Van Beers S, Van Benthem BHB, Walton C, et al. 2007. Impact of land-use change on dengue and malaria in northern Thailand. Ecohealth. 4(1):37-51. doi:10.1007/s10393007-0085-5.

Vega Rúa A, Okech BA. 2019. The spread of mosquito-borne diseases: A major and global public health problem. In: Olfactory Concepts of Insect Control - Alternative to insecticides. Springer Nature Switzerland. p. 1-27.

Verdonschot PFM, Besse-Lototskaya AA. 2014. Flight distance of mosquitoes (Culicidae): A metadata analysis to support the management of barrier zones around rewetted and newly constructed wetlands. Limnologica. 45:69-79. doi:10.1016/j.limno.2013.11.002.

Verhulst NO, Loonen JACM, Takken W. 2013. Advances in methods for colour marking of mosquitoes. Parasit Vectors. 6(1):1-7. doi:10.1186/1756-3305-6-200.

Villari P, Spielman A, Komar N, McDowell M, Timperi RJ. 1995. The economic burden imposed by a residual case of eastern encephalitis. Am J Trop Med Hyg. 52`(8-13).

vonHedemann N, Butterworth MK, Robbins P, Landau K, Morin CW. 2015. Visualizations of mosquito risk: A political ecology approach to understanding the territorialization of hazard control. Landsc Urban Plan. 142:159-169. doi:10.1016/j.landurbplan.2015.03.001.

Wada Y, Kawai S, Oda T, Miyagi I, Suenaga O, Nishigaki J, Omori N. 1969. Dispersal experiment of Culex tritaeniorhynchus in Nagasake area (preliminary report). Trop Med. 11(1):37-44.

Walter Reed Biosystematics Unit. 2020. Culex restuans Theobald, 1901. Culex restuans species page Walter Reed Biosyst Unit Website. [accessed 2020 Dec 13]. https://www.wrbu.si.edu/vectorspecies/mosquitoes/restuans.

Washino RK, Wood BL. 1994. Application of remote sensing to vector arthropod surveillance and control. Am J Trop Med Hyg. 50(134-144 (suppl.)).

Watson TM, Saul A, Kay BH. 2000. Aedes notoscriptus (Diptera: Culicidae) survival and dispersal estimated by mark-release-recapture in Brisbane, Queensland, Australia. J Med Entomol. 37(3):380-384. doi:10.1603/0022-2585(2000)037[0380:ANDCSA]2.0.CO;2.

Weaver S. 2005. Journal policy of aedine mosquito genera and subgenera. Am J Trop Med Hyg. 73(3):481. doi:10.1093/jme/tjv169.

Webb CE, Russell RC. 2019. Dispersal of the mosquito Aedes vigilax (Diptera : Culicidae) from urban estuarine wetlands in Sydney, Australia. (X):1-6. doi:10.1093/jme/tjz054.

Weih RCWJ, Riggan NDJ. 2010. Object-based classification vs. pixel-based classification: comparitive importance of multi-resolution imagery. Int Arch Photogramm Remote Sens 
Spat Inf Sci. 38(4/C7).

Weissman M. 2016. Mosquito of the Month: Aedes vexans - the Inland Floodwater Mosquito. Vector Dis Control Int.

Werner A, Storie CD, Storie J. 2014. Evaluating SAR-optical image fusions for urban LULC classification in Vancouver Canada. Can J Remote Sens. 40(4):278-290. doi:10.1080/07038992.2014.976700.

Westwood R, Westwood AR, Hooshmandi M, Pearson K, LaFrance K, Murray C. 2019. A field-validated species distribution model to support management of the critically endangered Poweshiek skipperling (Oarisma poweshiek) butterfly in Canada. Conserv Sci Pract.(March 2019):1-15. doi:10.1111/csp2.163.

Wiese D, Escalante AA, Murphy H, Henry KA, Gutierrez-Velez VH. 2019. Integrating environmental and neighborhood factors in MaxEnt modeling to predict species distributions: A case study of Aedes albopictus in southeastern Pennsylvania. PLoS One. 14(10):e0223821. doi:10.1371/journal.pone.0223821.

Wilkerson RC, Linton YM, Fonseca DM, Schultz TR, Price DC, Strickman DA. 2015. Making mosquito taxonomy useful: A stable classification of tribe Aedini that balances utility with current knowledge of evolutionary relationships. PLoS One. 10(7):1-26. doi:10.1371/journal.pone.0133602.

Wilson BG, Adams BJ, Karney BW. 1990. Bias in log-transformed frequency distributions. J Hydrol. 118:19-37. doi:10.1016/0022-1694(90)90248-V.

Winskill P, Carvalho DO, Capurro ML, Alphey L, Donnelly CA, McKemey AR. 2015. Dispersal of engineered male Aedes aegypti mosquitoes. PLoS Negl Trop Dis. 9(11):118. doi:10.1371/journal.pntd.0004156.

Winters AM, Eisen RJ, Lozano-Fuentes S, Moore CG, Pape WJ, Eisen L. 2008. Predictive spatial models for risk of West Nile virus exposure in eastern and western Colorado. Am J Trop Med Hyg. 79(4):581-590. doi:79/4/581 [pii].

Wong GKL, Jim CY. 2017. Urban-microclimate effect on vector mosquito abundance of tropical green roofs. Build Environ. 112:63-76. doi:10.1016/j.buildenv.2016.11.028.

Wood DM, Dang PT, Ellis RA. 1979. The Insects and Arachnids of Canada. Part 6. The Mosquitoes of Canada. 1st ed. Ottawa, Canada: Agriculture Canada.

World Health Organisation. 2019. Neglected tropical diseases: Mosquito-borne diseases.

Worobey JD, Fonseca C, Espinosa S, Healy S, Gaugler R. 2013. Child outdoor physical activity is reduced by prevalence of the Asian tiger mosquito Aedes albopictus. J Am Mosq Control Assoc. 29:78-80.

Yoo E-H, Chen D, Diao C, Russell C. 2016. The effects of weather and environmental factors on West Nile virus mosquito abundance in Greater Toronto Area. Earth Interact. 20(3).

Zar J. 2010. Biostatistical Analysis. 5th ed. Upper Saddle River, New Jersey: Prentice Hall. 
Zittra C, Vitecek S, Obwaller AG, Rossiter H, Eigner B, Zechmeister T, Waringer J, Fuehrer HP. 2017. Landscape structure affects distribution of potential disease vectors (Diptera: Culicidae). Parasit Vectors. 10(1):1-13. doi:10.1186/s13071-017-2140-6.

Zou L, Miller SN, Schmidtmann ET. 2006. Mosquito larval habitat mapping using remote sensing and GIS: implications of coalbed methane development and West Nile virus. J Med Entomol. 43(5):1034-1041.

Zwiebel LJ, Takken W. 2004. Olfactory regulation of mosquito-host interactions. Insect Biochem Mol Biol. 34(7):645-652. 


\section{APPENDICES}

Appendix I. List of mosquito species collected as larvae and/or as adults within the Winnipeg control zone (as of 2020).

Aedes (Ochlerotatus) abserratus

Aedes (Ochlerotatus) campestris

Aedes (Ochlerotatus) canadensis

Aedes (Ochlerotatus) communis

Aedes cinereus

Aedes (Ochlerotatus) diantaeus

Aedes (Ochlerotatus) dorsalis

Aedes (Ochlerotatus) euedes

Aedes (Ochlerotatus) excrucians

Aedes (Ochlerotatus) fitchii

Aedes (Ochlerotatus) flavescens

Aedes (Ochlerotatus) hendersoni

Aedes (Ochlerotatus) implicatus

Aedes (Ochlerotatus) intrudens

Aedes (Ochlerotatus) mercurator

Aedes (Ochlerotatus) nigromaculis

Aedes (Ochlerotatus) pionips

Aedes (Ochlerotatus) provocans

Aedes (Ochlerotatus) punctor

Aedes (Ochlerotatus) riparius

Aedes (Ochlerotatus) spencerii

Aedes (Ochlerotatus) sticticus

Aedes (Ochlerotatus) stimulans

Aedes (Ochlerotatus) triseriatus
(Felt and Young, 1904)

(Dyar and Knab, 1907)

(Theobald, 1901)

(De Geer, 1976)

Meigen, 1818

(Howard, Dyar and Knab, 1913)

(Meigen, 1830)

(Howard, Dyar and Knab, 1913)

(Walker, 1856)

(Felt and Young, 1904)

(Muller, 1764)

(Cockerell, 1918)

(Vockeroth, 1954)

(Dyar, 1919)

(Dyar, 1920)

(Ludlow, 1906)

(Dyar, 1919)

(Walker, 1848)

(Kirby, 1837)

(Dyar and Knab, 1907)

(Theobald, 1901)

(Meigen, 1838)

(Walker, 1848)

(Say, 1823)

\begin{tabular}{|c|c|}
\hline Aedes (Ochlerotatus) trivittatus & (Coquillett, 1902) \\
\hline Aedes vexans & (Meigen, 1830) \\
\hline Aedes (Ochlerotatus) ventrovittis & (Dyar, 1916) \\
\hline Anopheles earlei & Vargas, 1943 \\
\hline Anopheles punctipennis & (Say, 1823) \\
\hline Anopheles walkeri & (Theobald, 1901) \\
\hline Coquillettidia perturbans & (Walker, 1856) \\
\hline Culex restuans & Theobald, 1901 \\
\hline Culex tarsalis & Coquillett, 1896 \\
\hline Culex territans & Walker, 1856 \\
\hline Culiseta alaskaensis & (Ludlow, 1906) \\
\hline Culiseta impatiens & (Walker, 1848) \\
\hline Culiseta inornata & (Williston, 1893) \\
\hline Culiseta melanura & (Coquillett, 1902) \\
\hline Culiseta minnesotae & Barr, 1957 \\
\hline Culiseta morsitans & (Theobald, 1901) \\
\hline
\end{tabular}

Uranotaenia sapphirina
(Osten Sacken, 1868) 
Appendix II. Descriptions of all numbered trap locations and their immediate surroundings. Nearest waterway is specified in parentheses after distance $-\mathrm{A}=$ Assiniboine River, LS = La Salle River, R = Red River, S = Seine River and SC = Sturgeon Creek. Traps which were only operational in 2017 are shaded. All traps are NJLTs unless otherwise noted. Continued on next page.

\begin{tabular}{|c|c|c|c|c|c|c|c|}
\hline \multirow{2}{*}{$\begin{array}{c}\text { Trap } \\
\text { no. } \\
1\end{array}$} & \multirow{2}{*}{$\begin{array}{c}\begin{array}{c}\text { Distance from } \\
\text { release site }(\mathbf{k m})\end{array} \\
0.2\end{array}$} & \multirow{2}{*}{$\begin{array}{c}\text { Direction from } \\
\text { release site }\end{array}$} & \multicolumn{2}{|c|}{$\begin{array}{c}\text { Distance to nearest } \\
\text { waterway }(\mathbf{k m})\end{array}$} & \multirow{2}{*}{$\begin{array}{c}\text { Land cover } \\
\text { grass / herbaceous }\end{array}$} & \multicolumn{2}{|c|}{ Land use } \\
\hline & & & 0.45 & (LS) & & agricultural & tree nursery \\
\hline $2^{\mathrm{MLT}}$ & 0.4 & NNW & 0.22 & (LS) & tree canopy & residential & yard \\
\hline 3 & 1.0 & $\mathrm{E}$ & 0.25 & $(\mathrm{R})$ & tree canopy & residential & yard \\
\hline $4^{\mathrm{MLT}}$ & 1.3 & NNW & 0.15 & (R) & grass / herbaceous & recreational & golf course \\
\hline $5^{\text {MLT }}$ & 2.0 & SW & 0.16 & (LS) & tree canopy & recreational & campground \\
\hline $6^{\text {MLT }}$ & 2.1 & ENE & 0.85 & (R) & grass / herbaceous & agricultural & rural yard \\
\hline $7^{\mathrm{MLT}}$ & 2.5 & WNW & 1.65 & (LS) & grass / herbaceous & agricultural & near road \\
\hline 8 & 2.7 & SE & 0.10 & $(\mathrm{R})$ & tree canopy & residential & yard \\
\hline 9 & 3.1 & ESE & 0.15 & (R) & tree canopy & residential & yard \\
\hline 10 & 3.3 & SSE & 0.32 & (R) & grass / herbaceous & municipal & community centre \\
\hline 11 & 3.6 & $\mathrm{~N}$ & 0.05 & $(\mathrm{R})$ & tree canopy & residential & yard \\
\hline 12 & 3.7 & SSW & 1.30 & (LS) & grass / herbaceous & agricultural & rural yard \\
\hline 13 & 4.4 & NNE & 0.68 & $(\mathrm{R})$ & bare ground & commercial & garden \\
\hline 14 & 4.7 & SE & 0.56 & (R) & tree canopy & residential & yard \\
\hline 15 & 4.9 & NNW & 2.07 & (R) & grass / herbaceous & residential & yard \\
\hline 16 & 6.0 & NNE & 0.10 & (R) & tree canopy & recreational & open space \\
\hline 17 & 7.8 & ENE & 0.63 & $(\mathrm{~S})$ & tree canopy & agricultural & rural yard \\
\hline 18 & 8.1 & NNE & 1.5 & (R) & grass / herbaceous & municipal & fire station \\
\hline 19 & 8.7 & NNW & 2.35 & (R) & grass / herbaceous & municipal & fire station \\
\hline 20 & 9.3 & $\mathrm{~N}$ & 0.18 & $\mathrm{Y}$ & tree canopy & recreational & open space \\
\hline 21 & 9.9 & NNE & 0.46 & $(\mathrm{~S})$ & grass / herbaceous & residential & yard \\
\hline 22 & 11.8 & $\mathrm{NE}$ & 2.40 & $(\mathrm{~S})$ & grass / herbaceous & residential & yard \\
\hline 23 & 12.7 & NNW & 2.20 & (A) & grass / herbaceous & residential & yard \\
\hline 24 & 13.1 & NNE & 0.17 & (S) & tree canopy & recreational & golf course \\
\hline 25 & 13.5 & WNW & 9.89 & (A) & grass / herbaceous & residential & yard \\
\hline 26 & 14.2 & NNE & 2.30 & $(\mathrm{~S})$ & grass / herbaceous & residential & yard \\
\hline
\end{tabular}


Appendix II. Continued from previous page.

\begin{tabular}{|c|c|c|c|c|c|c|c|}
\hline \multirow{2}{*}{$\begin{array}{r}\begin{array}{c}\text { Trap } \\
\text { no. }\end{array} \\
27\end{array}$} & \multirow{2}{*}{$\begin{array}{c}\begin{array}{c}\text { Distance from } \\
\text { release site (km) }\end{array} \\
14.6\end{array}$} & \multirow{2}{*}{$\begin{array}{c}\begin{array}{c}\text { Direction from } \\
\text { release site }\end{array} \\
\mathrm{N}\end{array}$} & \multicolumn{2}{|c|}{$\begin{array}{c}\text { Distance to nearest } \\
\text { waterway }(\mathbf{k m})\end{array}$} & \multirow{2}{*}{$\begin{array}{l}\text { Land cover } \\
\text { grass / herbaceous }\end{array}$} & \multirow{2}{*}{\multicolumn{2}{|c|}{$\begin{array}{r}\text { Land use } \\
\text { yard }\end{array}$}} \\
\hline & & & 0.50 & $(\mathrm{R})$ & & & \\
\hline 28 & 15.4 & $\mathrm{~N}$ & 0.25 & (A) & grass / herbaceous & residential & yard \\
\hline 29 & 15.5 & NW & 1.00 & (A) & grass / herbaceous & residential & yard \\
\hline 30 & 15.5 & NNW & 0.45 & (A) & tree canopy & recreational & open space \\
\hline 31 & 15.7 & NE & 6.75 & (S) & grass / herbaceous & agricultural & cemetery \\
\hline 32 & 16.0 & NNW & 0.45 & (A) & grass / herbaceous & residential & yard \\
\hline 33 & 16.6 & NW & 3.05 & (A) & bare ground & municipal & water plant \\
\hline 34 & 17.8 & $\mathrm{~N}$ & 0.83 & (R) & bare ground & municipal & government office \\
\hline 35 & 17.9 & NNE & 4.57 & $(\mathrm{~S})$ & grass / herbaceous & residential & yard \\
\hline 36 & 19.4 & NW & 1.14 & (SC) & grass / herbaceous & residential & yard \\
\hline 37 & 19.7 & NNW & 4.47 & (A) & grass / herbaceous & municipal & cemetery \\
\hline 38 & 20.4 & $\mathrm{~N}$ & 4.50 & (R) & grass / herbaceous & residential & yard \\
\hline 39 & 20.8 & NNE & 6.46 & $(\mathrm{R})$ & grass / herbaceous & recreational & open space \\
\hline 40 & 21.0 & NNE & 3.00 & (R) & grass / herbaceous & residential & yard \\
\hline 41 & 21.4 & NNE & 8.75 & (R) & grass / herbaceous & residential & yard \\
\hline 42 & 21.5 & $\mathrm{~N}$ & 0.19 & (R) & tree canopy & recreational & open space \\
\hline 43 & 21.5 & NW & 0.85 & (A) & grass / herbaceous & commercial & car dealership \\
\hline 44 & 22.3 & $\mathrm{~N}$ & 0.53 & $(\mathrm{R})$ & tree canopy & recreational & open space \\
\hline 45 & 23.3 & NNE & 1.65 & $(\mathrm{R})$ & grass / herbaceous & residential & yard \\
\hline 46 & 23.7 & $\mathrm{~N}$ & 2.91 & $(\mathrm{R})$ & grass / herbaceous & residential & yard \\
\hline 47 & 23.9 & $\mathrm{~N}$ & 3.35 & (R) & grass / herbaceous & municipal & sewage plant \\
\hline 48 & 24.0 & NW & 0.13 & (A) & tree canopy & residential & yard \\
\hline 49 & 25.0 & NNW & 7.35 & $(\mathrm{SC})$ & bare ground & municipal & open space \\
\hline 50 & 26.4 & NNE & 0.65 & $(\mathrm{R})$ & grass / herbaceous & residential & yard \\
\hline 51 & 27.9 & $\mathrm{~N}$ & 0.12 & (R) & grass / herbaceous & residential & yard \\
\hline $52 *$ & 7.8 & $\mathrm{~N}$ & 0.10 & $(\mathrm{R})$ & grass / herbaceous & residential & yard \\
\hline $53 *$ & 20.8 & NW & 0.15 & $(\mathrm{~A})$ & grass / herbaceous & recreational & golf course \\
\hline
\end{tabular}

*trap was only operational in the MRR study period of 2016. 
Appendix III. Summary of estimated numbers of marked mosquitoes released per rearing pool by release day (2016).

Number of marked mosquitoes was based on visual estimates of minimum and maximum.

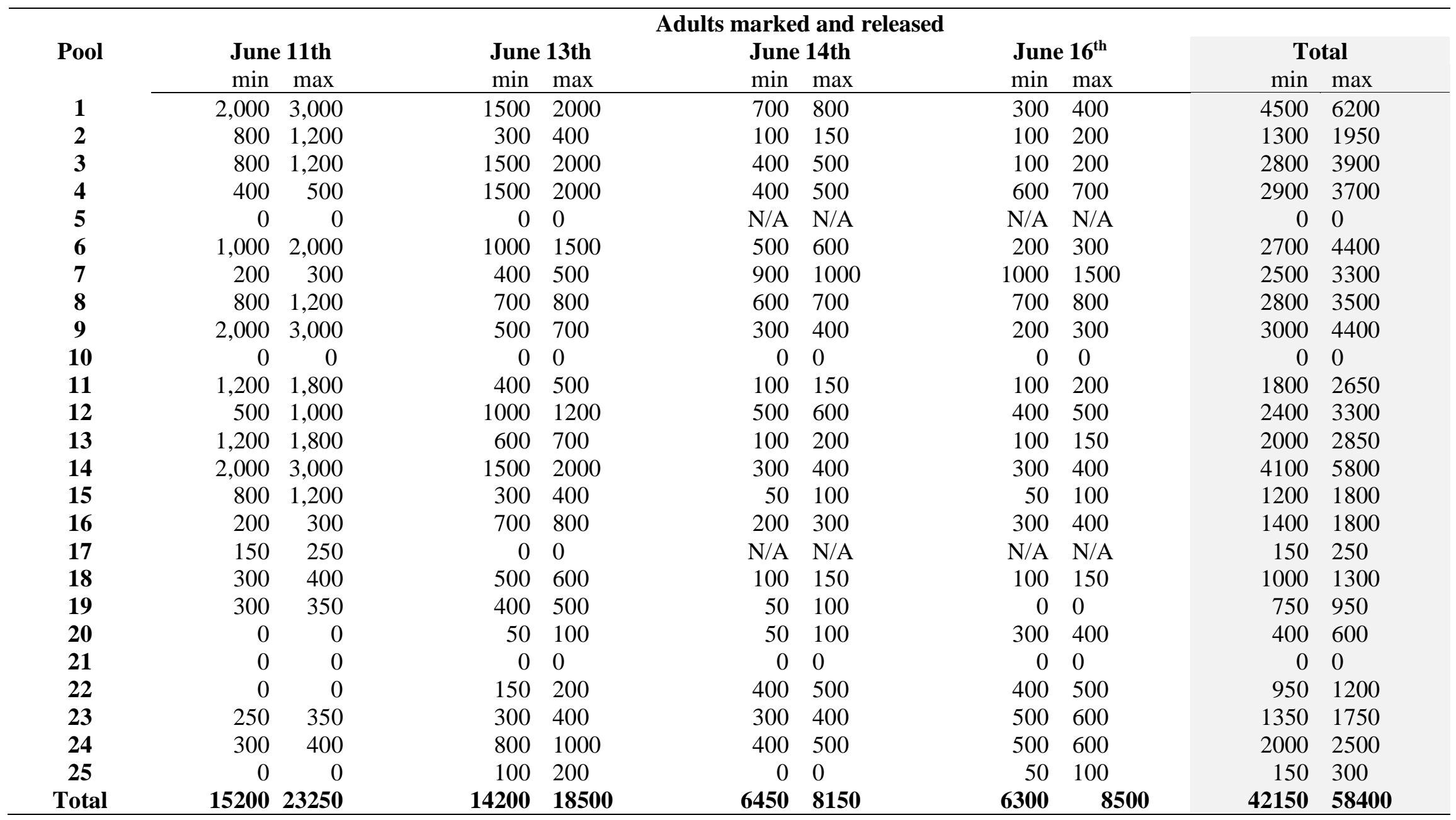


Appendix IV. Summary of marked mosquitoes released per rearing pool by release day (2017). Number of marked mosquitoes was based on manual counts at the time of release, except for those on August $1^{\text {st }}$, which were based on counts from photos of the screened enclosures at the time of release.

\begin{tabular}{|c|c|c|c|c|c|}
\hline \multirow[b]{2}{*}{ Pool } & \multicolumn{4}{|c|}{ Adults marked and released } & \multirow[b]{2}{*}{ Total } \\
\hline & August $1^{\text {st }}$ & August $8^{\text {th }}$ & August $14^{\text {th }}$ & August $16^{\text {th }}$ & \\
\hline 1 & 253 & 0 & 154 & 0 & 407 \\
\hline 2 & 0 & 0 & 136 & 547 & 683 \\
\hline 3 & 267 & 0 & 226 & 198 & 691 \\
\hline 4 & 125 & 0 & 247 & 303 & 675 \\
\hline 5 & 0 & 538 & 0 & 726 & 1,264 \\
\hline 6 & 0 & 0 & 0 & 0 & 0 \\
\hline 7 & 208 & 1,899 & 536 & 1,644 & 4,287 \\
\hline 8 & 0 & 0 & 0 & 0 & 0 \\
\hline 9 & 0 & 811 & 1,169 & 1,079 & 3,059 \\
\hline 10 & 0 & 218 & 173 & 1,067 & 1,458 \\
\hline 11 & 0 & 0 & 0 & 393 & 393 \\
\hline 12 & 245 & 1,086 & 617 & 1,110 & 3,058 \\
\hline 13 & 61 & 0 & 0 & 465 & 526 \\
\hline 14 & 0 & 982 & 748 & 0 & 1,730 \\
\hline 15 & 221 & 0 & 0 & 0 & 221 \\
\hline 16 & 164 & 232 & 104 & 0 & 500 \\
\hline 17 & 0 & 2,449 & 432 & 776 & 3,657 \\
\hline 18 & 0 & 0 & 188 & 588 & 776 \\
\hline 19 & 208 & 0 & 89 & 0 & 297 \\
\hline 20 & 0 & 0 & 0 & 0 & 0 \\
\hline 21 & 0 & 0 & 208 & 1,099 & 1,307 \\
\hline 22 & 0 & 832 & 190 & 972 & 1,994 \\
\hline 23 & 0 & 0 & 43 & 277 & 320 \\
\hline 24 & 0 & 0 & 216 & 140 & 356 \\
\hline 25 & 0 & 453 & 247 & 564 & 1,264 \\
\hline Total & 1,752 & 9,500 & 5,723 & 11,948 & 28,932 \\
\hline
\end{tabular}


Appendix V. Precipitation, air temperature, relative humidity, and wind velocity recorded at Winnipeg International Airport during the markrelease-recapture study period in 2016. Mean nightly variables were calculated between 20:00 h of the previous day and 5:00 of the corresponding day shown. Continued on the next page.

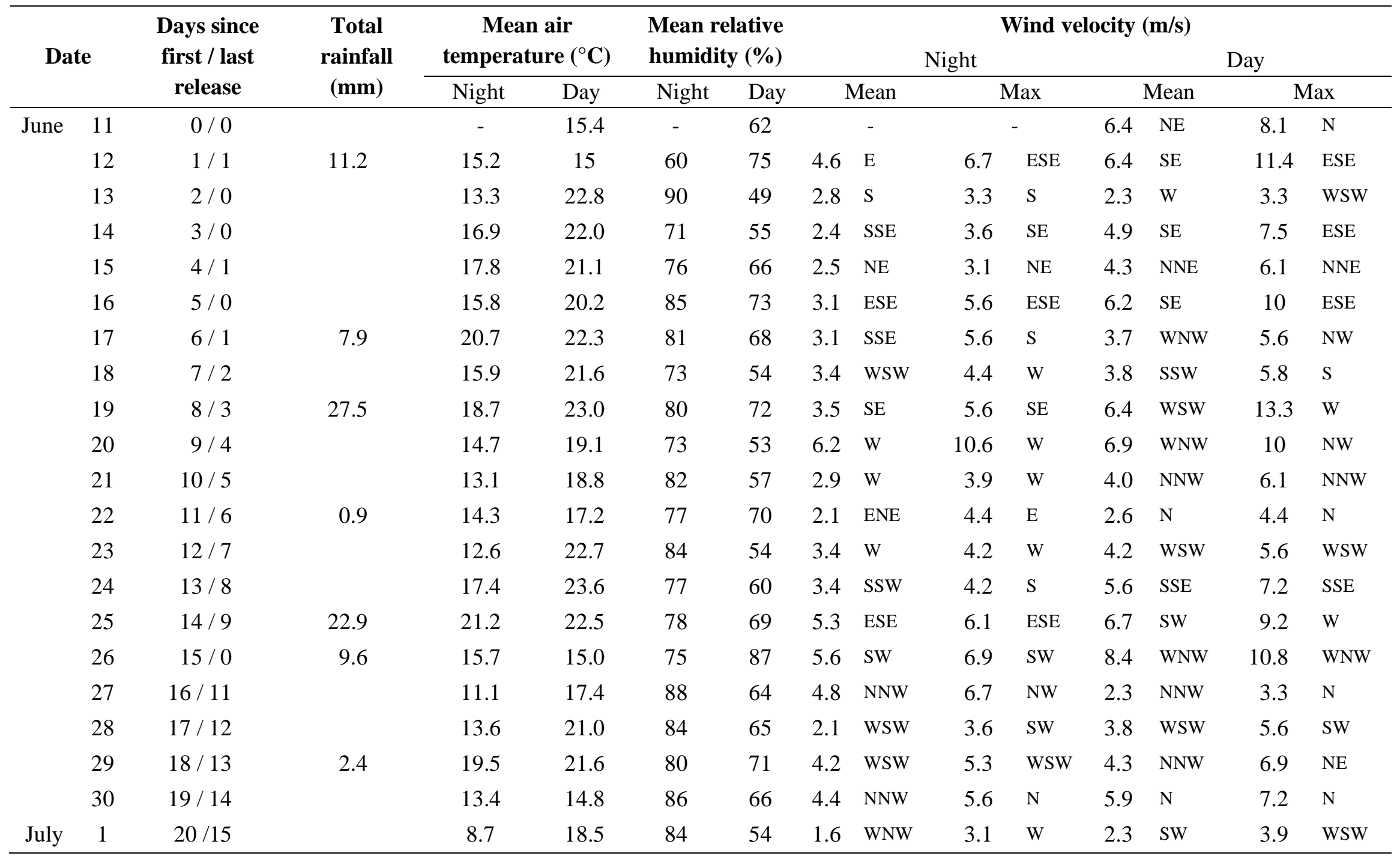


Appendix V. Continued from previous page.

\begin{tabular}{|c|c|c|c|c|c|c|c|c|c|c|c|c|c|c|c|}
\hline \multirow{2}{*}{\multicolumn{2}{|c|}{ Date }} & \multirow{3}{*}{$\begin{array}{c}\text { Days since } \\
\text { last release } \\
21 / 16\end{array}$} & \multirow{3}{*}{$\begin{array}{l}\text { Total rainfall } \\
\qquad(\mathrm{mm})\end{array}$} & \multicolumn{2}{|c|}{$\begin{array}{c}\text { Mean air } \\
\text { temperature }\left({ }^{\circ} \mathrm{C}\right)\end{array}$} & \multicolumn{2}{|c|}{$\begin{array}{l}\text { Mean relative } \\
\text { humidity }(\%)\end{array}$} & \multicolumn{8}{|c|}{ Wind velocity $(\mathrm{m} / \mathrm{s})$} \\
\hline & & & & \multirow{2}{*}{$\begin{array}{c}\text { Night } \\
15.5\end{array}$} & \multirow{2}{*}{$\frac{\text { Day }}{21.8}$} & \multirow{2}{*}{$\frac{\text { Night }}{72}$} & \multirow{2}{*}{$\frac{\text { Day }}{51}$} & \multicolumn{2}{|c|}{ Mean } & \multicolumn{2}{|c|}{$\operatorname{Max}$} & \multicolumn{2}{|c|}{ Mean } & \multicolumn{2}{|c|}{ Max } \\
\hline July & 2 & & & & & & & 2.0 & SSE & 2.5 & SSE & 3.5 & SSE & 4.4 & $\mathrm{~S}$ \\
\hline & 3 & $22 / 17$ & 2.1 & 17.7 & 20.8 & 74 & 78 & 2.7 & SSE & 3.3 & SSE & 4.0 & $\mathrm{SE}$ & 5.3 & $\mathrm{~S}$ \\
\hline & 4 & $23 / 18$ & 22.2 & 18.7 & 23.6 & 81 & 74 & 4.5 & S & 5.3 & S & 4.8 & SW & 9.4 & WSW \\
\hline & 5 & $24 / 19$ & 0.8 & 16.4 & 20.2 & 89 & 79 & 2.1 & WSW & 3.9 & $\mathrm{~W}$ & 2.8 & $\mathrm{~W}$ & 4.2 & WSW \\
\hline & 6 & $25 / 20$ & 0.2 & 13.7 & 18.9 & 94 & 70 & 2.1 & WNW & 2.8 & NW & 3.3 & NNW & 4.7 & NNW \\
\hline & 7 & $26 / 21$ & & 15.4 & 19.0 & 81 & 70 & 2.6 & ENE & 3.6 & $\mathrm{NE}$ & 3.5 & ENE & 4.2 & $\mathrm{NE}$ \\
\hline & 8 & $27 / 22$ & & 15.3 & 21.4 & 90 & 69 & 2.8 & NNW & 4.2 & NNE & 3.3 & $\mathrm{~N}$ & 5 & $\mathrm{~N}$ \\
\hline & 9 & $28 / 23$ & 2 & 15.3 & 21.5 & 90 & 73 & 1.1 & ENE & 2.2 & $\mathrm{~S}$ & 3.5 & SSE & 5.3 & $\mathrm{SE}$ \\
\hline & 10 & $29 / 24$ & & 18.6 & 22.3 & 89 & 80 & 3.3 & SE & 4.7 & SSE & 3.7 & SSE & 5.6 & SSE \\
\hline & 11 & $30 / 25$ & 3.9 & 21.3 & 22.6 & 84 & 80 & 4.1 & SE & 6.4 & SSE & 3.5 & $\mathrm{SE}$ & 6.4 & ESE \\
\hline & 12 & $31 / 26$ & 7.8 & 19.0 & 19.3 & 91 & 81 & 4.9 & S & 7.5 & S & 6.6 & SW & 8.9 & SW \\
\hline & 13 & $32 / 27$ & 5.4 & 17.3 & 17.8 & 92 & 90 & 8.3 & WSW & 10.8 & $\mathrm{~W}$ & 7.1 & WNW & 8.3 & WNW \\
\hline & 14 & 33 / 28 & 0.2 & 14.9 & 19.0 & 91 & 65 & 5.3 & NNW & 6.1 & NNW & 4.8 & NNW & 6.1 & NNW \\
\hline & 15 & 34 / 29 & 0.2 & 11.5 & 19.9 & 87 & 54 & 2.1 & NNW & 3.1 & NNW & 1.8 & $\mathrm{~N}$ & 2.8 & ENE \\
\hline & 16 & $35 / 30$ & 0.7 & 14.5 & 20.3 & 82 & 78 & 1.8 & $\mathrm{~S}$ & 2.8 & S & 3.7 & SSW & 5.6 & $\mathrm{~S}$ \\
\hline & 17 & 36 / 31 & 5.5 & 15.2 & 20.0 & 95 & 74 & 1.5 & SSW & 2.2 & SSW & 4.9 & NW & 8.1 & $\mathrm{~N}$ \\
\hline & 18 & 37 / 32 & NA & 13.6 & 19.8 & 85 & 64 & 1.9 & NNE & 3.6 & $\mathrm{NE}$ & 1.7 & $\mathrm{SE}$ & 3.6 & $\mathrm{NE}$ \\
\hline & 19 & $38 / 33$ & & 17.3 & 24.5 & 82 & 72 & 3.6 & SSE & 4.2 & SSE & 5.3 & SSE & 7.8 & S \\
\hline & 20 & 39 / 34 & 19.5 & 22.1 & 27.4 & 81 & 76 & 6.4 & $\mathrm{~S}$ & 9.4 & S & 3.9 & SSW & 6.4 & $\mathrm{~S}$ \\
\hline & 21 & $40 / 35$ & & 18.0 & 25.6 & 91 & 63 & 4.3 & SW & 10.0 & $\mathrm{NE}$ & 5.4 & $\mathrm{~W}$ & 8.3 & W \\
\hline & 22 & $41 / 36$ & & 20.4 & 24.2 & 79 & 64 & 4.2 & WSW & 5.6 & WSW & 4.4 & NW & 5.6 & WNW \\
\hline & 23 & 42 / 37 & 12.2 & 19.0 & 20.9 & 80 & 81 & 2.3 & ESE & 5.3 & $\mathrm{SE}$ & 5.2 & $\mathrm{SE}$ & 6.7 & SE \\
\hline & 24 & 43 / 38 & & 18.2 & 21.7 & 92 & 71 & 4.6 & WSW & 5.6 & $\mathrm{~W}$ & 6.8 & WNW & 8.6 & WNW \\
\hline & 25 & 44 / 39 & & 16.5 & 24.3 & 91 & 63 & 2.8 & WSW & 3.6 & WSW & 3.2 & WSW & 4.2 & W \\
\hline & & Total & 165.1 & & & & & & & & & & & & \\
\hline
\end{tabular}


Appendix VI. Precipitation, air temperature, relative humidity, and wind velocity recorded at Winnipeg International Airport during the markrelease-recapture study period in 2017. Mean nightly variables were calculated between 20:00 h of the previous day and 5:00 of the corresponding day shown. Continued on the next page.

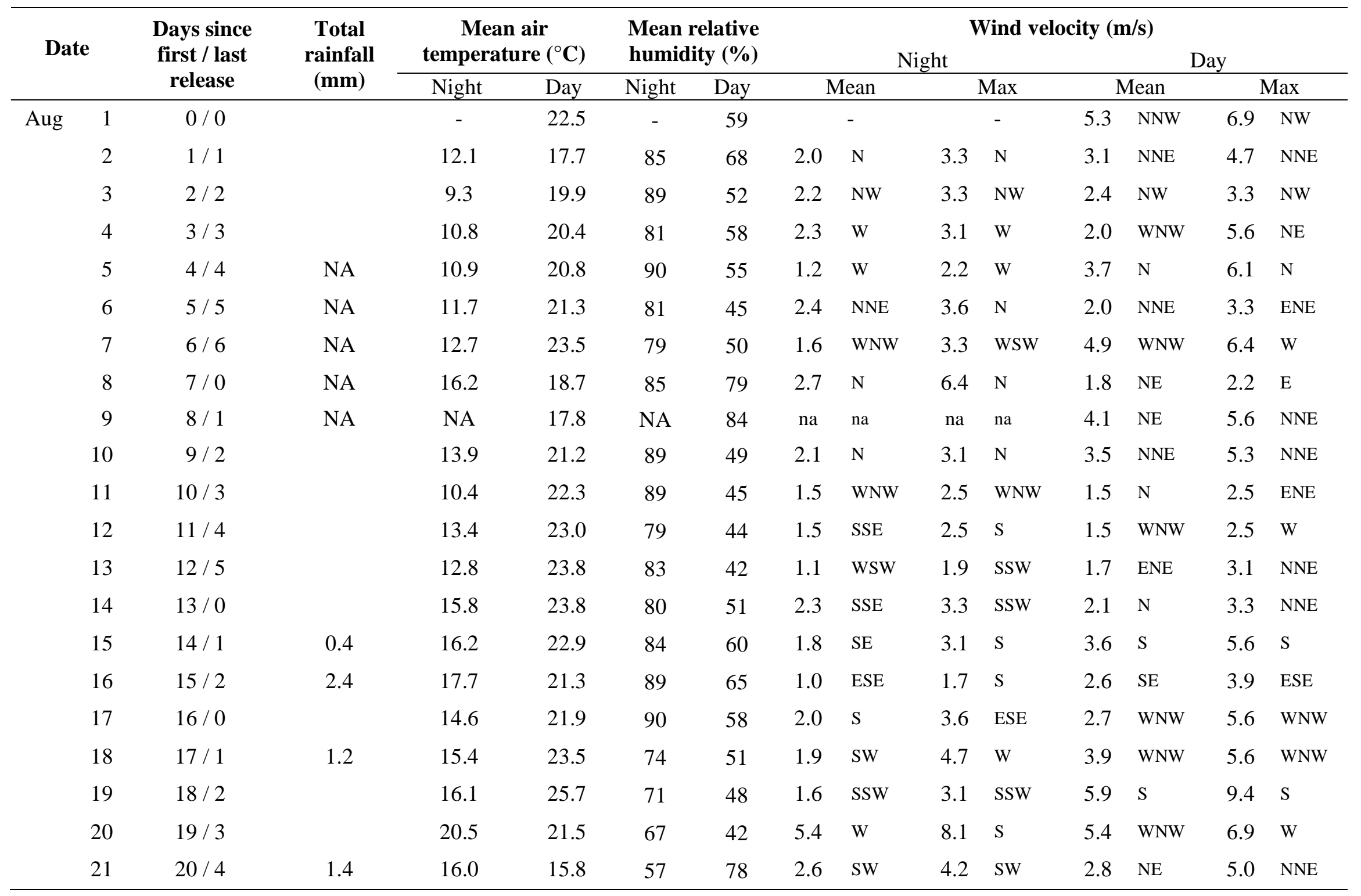


Appendix VI. Continued from previous page.

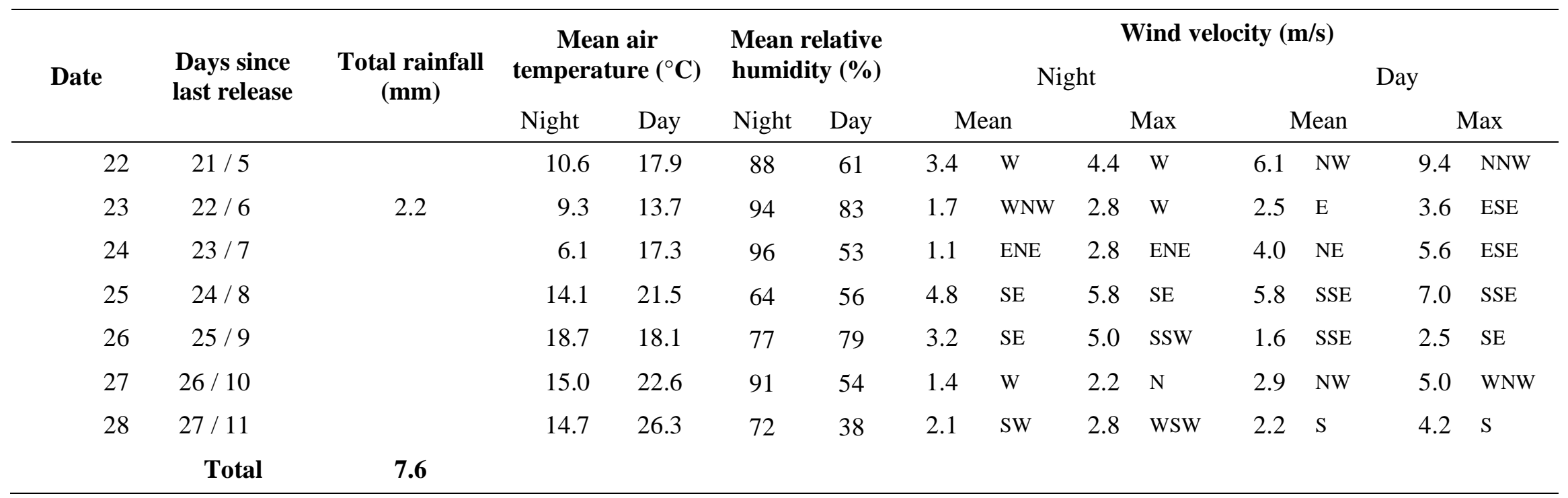


Appendix VII. Daily breakdown of recaptured mosquitoes in 2016 by minimum and maximum days since release. All traps collections were taken every day except June $28^{\text {th }}$, July $1^{\text {st }}$ and $3^{\text {rd }}$. Releases were on June $11^{\text {th }}, 13^{\text {th }}, 14^{\text {th }}$ and $16^{\text {th }}$.

\begin{tabular}{|c|c|c|c|c|c|c|c|}
\hline Month & Date & $\begin{array}{c}\text { Days since } \\
\text { first/last release }\end{array}$ & $\begin{array}{l}\text { Females } \\
\text { recaptured }\end{array}$ & $\begin{array}{c}\text { Males } \\
\text { recaptured }\end{array}$ & Daily total & Cumulative total & $\begin{array}{l}\text { Cumulative } \% \text { of total } \\
\text { recaptured }\end{array}$ \\
\hline \multirow[t]{17}{*}{ June } & 14 & $3 / 0$ & 2 & 1 & 3 & 3 & 3.8 \\
\hline & 15 & $4 / 1$ & 4 & & 4 & 7 & 8.9 \\
\hline & 16 & $5 / 0$ & & 1 & 1 & 8 & 10.1 \\
\hline & 17 & $6 / 1$ & 10 & 4 & 14 & 22 & 27.8 \\
\hline & 18 & $7 / 2$ & 7 & 1 & 8 & 30 & 38.0 \\
\hline & 19 & $8 / 3$ & 2 & 1 & 3 & 33 & 41.8 \\
\hline & 20 & $9 / 4$ & & & & 33 & 41.8 \\
\hline & 21 & $10 / 5$ & 1 & 1 & 2 & 35 & 44.3 \\
\hline & 22 & $11 / 6$ & & & & 35 & 44.3 \\
\hline & 23 & $12 / 7$ & & & & 35 & 44.3 \\
\hline & 24 & $13 / 8$ & & & & 35 & 44.3 \\
\hline & 25 & $14 / 9$ & 4 & & 4 & 39 & 48.1 \\
\hline & 26 & $15 / 10$ & 2 & & 2 & 41 & 51.9 \\
\hline & 27 & $16 / 11$ & 1 & & 1 & 42 & 53.2 \\
\hline & 28 & $17 / 12$ & 2 & & 2 & 44 & 55.7 \\
\hline & 29 & $18 / 13$ & 2 & & 2 & 46 & 58.2 \\
\hline & 30 & $19 / 14$ & 4 & & 4 & 50 & 63.3 \\
\hline \multirow[t]{4}{*}{ July } & 1 & $20 / 15$ & na & na & na & 50 & 63.3 \\
\hline & 2 & $21 / 16$ & 16 & 3 & 19 & 69 & 87.3 \\
\hline & 3 & $22 / 17$ & na & na & na & 69 & 87.3 \\
\hline & 4 & $23 / 18$ & 1 & & 1 & 70 & 88.6 \\
\hline
\end{tabular}


Appendix VII. Continued from previous page.

\begin{tabular}{|c|c|c|c|c|c|c|c|}
\hline Month & Date & $\begin{array}{c}\text { Days since } \\
\text { first/last release }\end{array}$ & $\begin{array}{l}\text { Females } \\
\text { recaptured }\end{array}$ & $\begin{array}{c}\text { Males } \\
\text { recaptured }\end{array}$ & Daily total & Cumulative total & $\begin{array}{c}\text { Cumulative } \% \text { of total } \\
\text { recaptured }\end{array}$ \\
\hline \multirow[t]{21}{*}{ July } & 5 & $24 / 19$ & & & & 70 & 88.6 \\
\hline & 6 & $25 / 20$ & 3 & 2 & 5 & 75 & 94.9 \\
\hline & 7 & $26 / 21$ & 1 & & 1 & 76 & 96.2 \\
\hline & 8 & $27 / 22$ & 1 & & 1 & 77 & 97.4 \\
\hline & 9 & $28 / 23$ & & & & 77 & 97.4 \\
\hline & 10 & $29 / 24$ & & & & 77 & 97.4 \\
\hline & 11 & $30 / 25$ & 1 & & 1 & 78 & 98.7 \\
\hline & 12 & $31 / 26$ & & & & 78 & 98.7 \\
\hline & 13 & $33 / 27$ & & & & 78 & 98.7 \\
\hline & 14 & $33 / 28$ & & & & 78 & 98.7 \\
\hline & 15 & $34 / 29$ & & & & 78 & 98.7 \\
\hline & 16 & $35 / 30$ & & & & 78 & 98.7 \\
\hline & 17 & $36 / 31$ & & & & 78 & 98.7 \\
\hline & 18 & 37 / 32 & & & & 78 & 98.7 \\
\hline & 19 & $38 / 33$ & & & & 78 & 98.7 \\
\hline & 20 & $39 / 34$ & & & & 78 & 98.7 \\
\hline & 21 & $40 / 35$ & & & & 78 & 98.7 \\
\hline & 22 & $41 / 36$ & & & & 78 & 98.7 \\
\hline & 23 & $42 / 37$ & & & & 78 & 98.7 \\
\hline & 24 & 43 / 38 & 1 & & 1 & 79 & 100 \\
\hline & 25 & 44 / 39 & & & & 79 & 100 \\
\hline
\end{tabular}


Appendix VIII. Number of marked and unmarked mosquitoes captured per $2 \mathrm{~km}$ radius from the release site from June $13^{\text {th }}$ to July $25^{\text {th }}, 2016$. Numbers of total mosquitoes trapped exclude those recaptured. Continued on the next page.

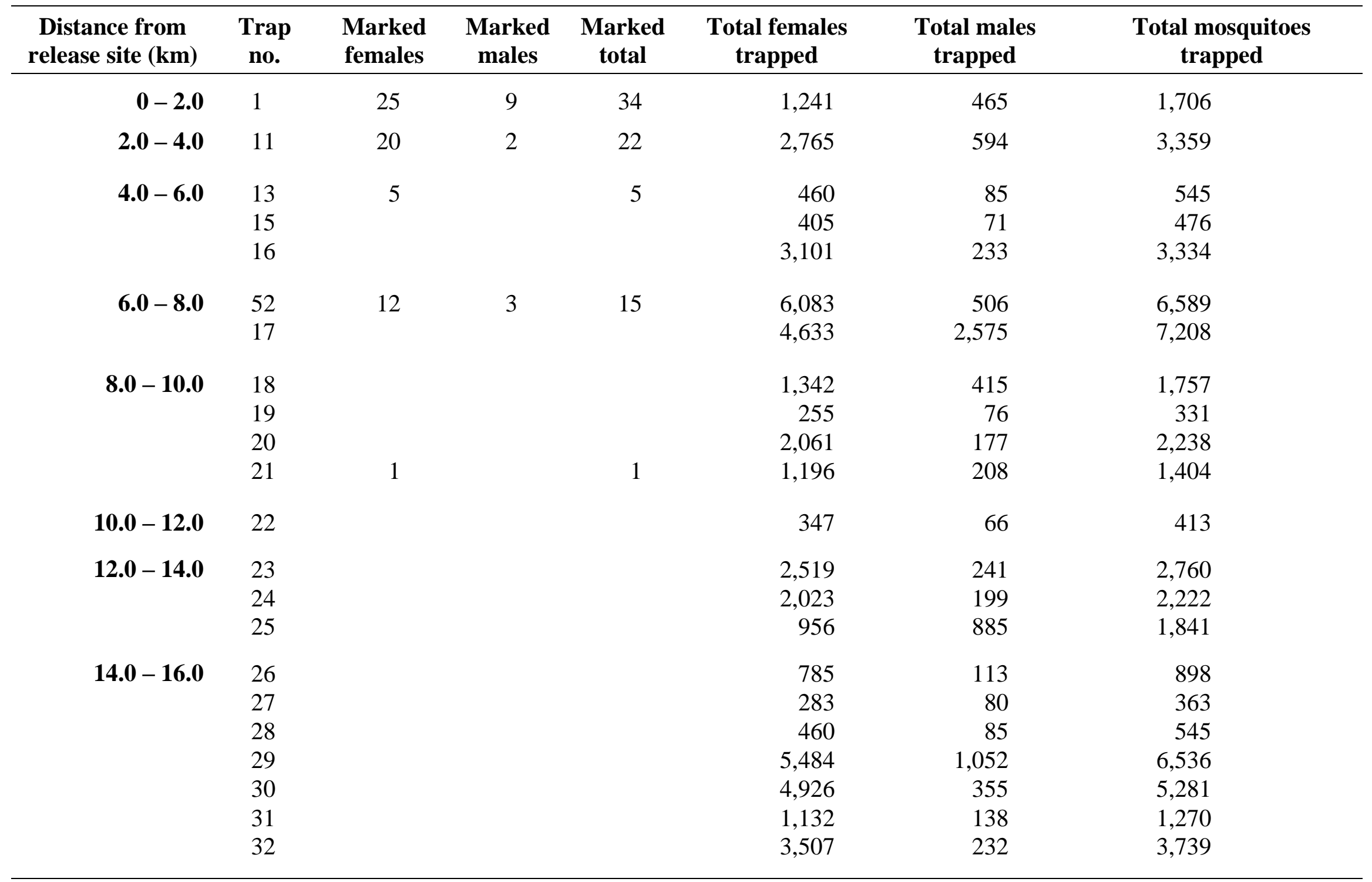


Appendix VIII. Continued from previous page.

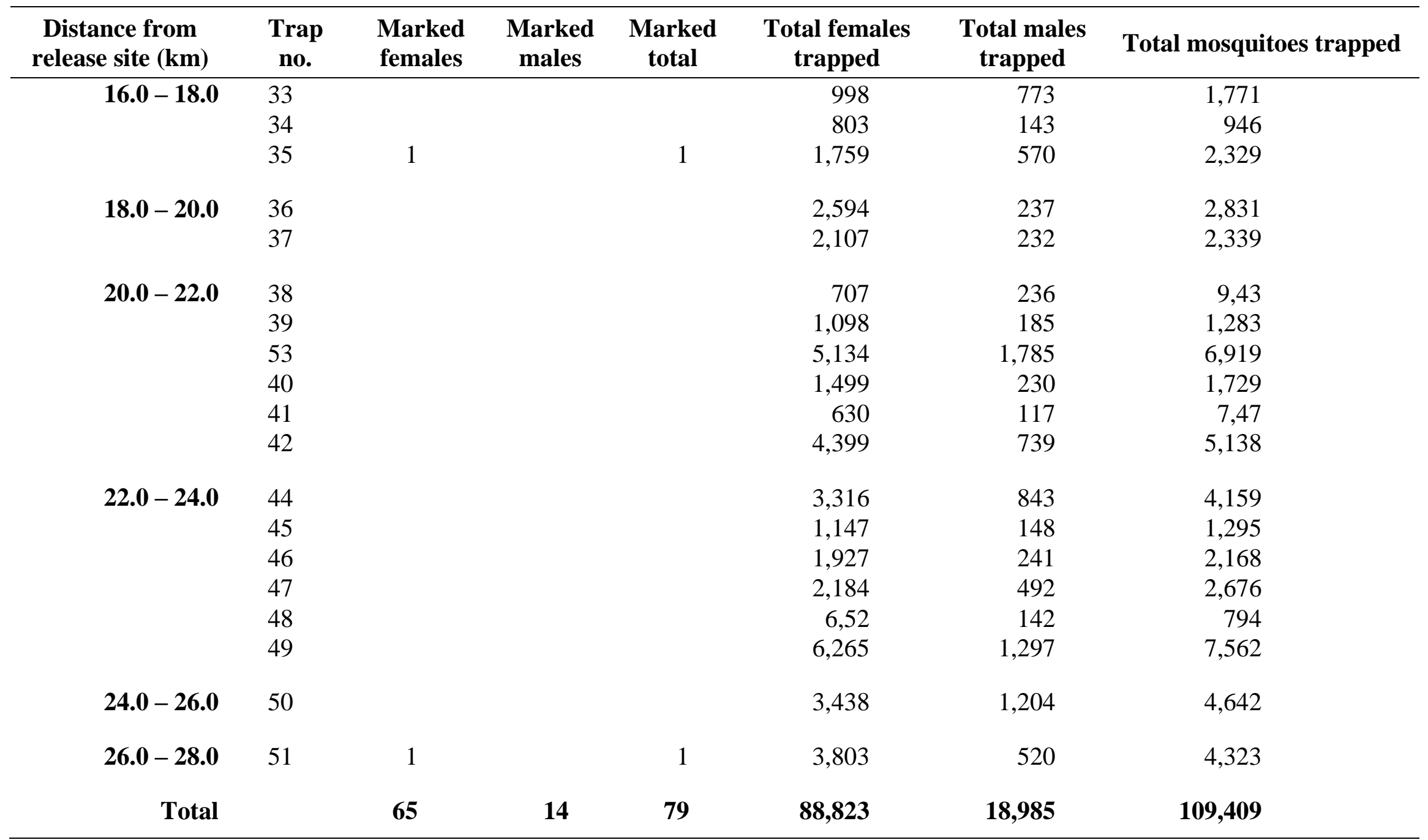


Appendix IX. List of recaptured mosquitoes in 2016, ordered by date, trap location, species, and sex. Blank entries follow the value of the most recent previous entry. Continued on the next page.

\begin{tabular}{|c|c|c|c|c|c|c|c|c|c|}
\hline \multirow[t]{2}{*}{ 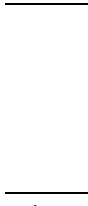 } & \multirow[t]{2}{*}{ Month } & \multirow[t]{2}{*}{ Day } & \multirow{2}{*}{$\begin{array}{c}\text { Days since } \\
\text { first / last } \\
\text { release }\end{array}$} & \multirow{2}{*}{$\begin{array}{l}\text { Trap } \\
\text { no. }\end{array}$} & \multirow{2}{*}{$\begin{array}{l}\text { km from } \\
\text { release } \\
\text { site }\end{array}$} & \multicolumn{2}{|c|}{$\begin{array}{l}\text { km divided } \\
\text { by no. days }\end{array}$} & \multirow[t]{2}{*}{ Species } & \multirow[t]{2}{*}{ Sex } \\
\hline & & & & & & Min. & Max. & & \\
\hline 1 & June & 14 & $3 / 0$ & 1 & 0.2 & 0.07 & 0.2 & Ae. vexans & $\mathrm{F}$ \\
\hline 2 & & 14 & $3 / 0$ & & & & & Ae. vexans & M \\
\hline 3 & & 14 & $3 / 0$ & 11 & 3.6 & 1.20 & 3.60 & Ae. vexans & $\mathrm{F}$ \\
\hline 4 & & 15 & $4 / 1$ & & & 0.90 & 3.60 & Ae. vexans & $\mathrm{F}$ \\
\hline 5 & & 15 & $4 / 1$ & & & & & Ae. vexans & $\mathrm{F}$ \\
\hline 6 & & 15 & $4 / 1$ & & & & & Ae. vexans & $\mathrm{F}$ \\
\hline 7 & & 15 & $4 / 1$ & & & & & Ae. vexans & $\mathrm{F}$ \\
\hline 8 & & 16 & $5 / 0$ & 1 & 0.2 & 0.04 & 0.10 & Ae. vexans & M \\
\hline 9 & & 17 & $6 / 1$ & & & 0.03 & 0.20 & Ae. vexans & $\mathrm{F}$ \\
\hline 10 & & 17 & $6 / 1$ & & & & & Ae. vexans & $\mathrm{F}$ \\
\hline 11 & & 17 & $6 / 1$ & & & & & Ae. vexans & $\mathrm{F}$ \\
\hline 12 & & 17 & $6 / 1$ & & & & & Ae. vexans & $\mathrm{F}$ \\
\hline 13 & & 17 & $6 / 1$ & & & & & Ae. vexans & M \\
\hline 14 & & 17 & $6 / 1$ & & & & & Ae. vexans & M \\
\hline 15 & & 17 & $6 / 1$ & 11 & 3.6 & 0.60 & 3.60 & Ae. vexans & $\mathrm{F}$ \\
\hline 16 & & 17 & $6 / 1$ & & & & & Ae. vexans & $\mathrm{F}$ \\
\hline 17 & & 17 & $6 / 1$ & & & & & Ae. vexans & $\mathrm{F}$ \\
\hline 18 & & 17 & $6 / 1$ & 52 & 7.8 & 1.30 & 7.80 & Ae. vexans & $\mathrm{F}$ \\
\hline 19 & & 17 & $6 / 1$ & & & & & Ae. vexans & $\mathrm{F}$ \\
\hline 20 & & 17 & $6 / 1$ & & & & & Ae. vexans & M \\
\hline 21 & & 17 & $6 / 1$ & & & & & Ae. vexans & M \\
\hline 22 & & 17 & $6 / 1$ & 21 & 9.9 & 1.65 & 9.90 & Ae. vexans & $\mathrm{F}$ \\
\hline 23 & & 18 & $7 / 2$ & 1 & 0.2 & 0.03 & 0.10 & Ae. vexans & $\mathrm{F}$ \\
\hline 24 & & 18 & $7 / 2$ & & & & & Ae. vexans & $\mathrm{F}$ \\
\hline 25 & & 18 & $7 / 2$ & & & & & Ae. vexans & $\mathrm{F}$ \\
\hline 26 & & 18 & $7 / 2$ & & & & & Ae. vexans & M \\
\hline 27 & & 18 & $7 / 2$ & 11 & 3.6 & 0.51 & 1.80 & Ae. vexans & $\mathrm{F}$ \\
\hline 28 & & 18 & $7 / 2$ & & & & & Ae. vexans & $\mathrm{F}$ \\
\hline 29 & & 18 & $7 / 2$ & & & & & Ae. vexans & $\mathrm{F}$ \\
\hline 30 & & 18 & $7 / 2$ & 13 & 4.4 & 0.63 & 2.20 & Ae. vexans & $\mathrm{F}$ \\
\hline 31 & & 19 & $8 / 3$ & 11 & 3.6 & 0.45 & 1.20 & Ae. vexans & $\mathrm{F}$ \\
\hline 32 & & 19 & $8 / 3$ & & & & & Ae. vexans & M \\
\hline 33 & & 19 & $8 / 3$ & 52 & 7.8 & 0.98 & 2.60 & Ae. vexans & $\mathrm{F}$ \\
\hline 34 & & 21 & $10 / 5$ & 1 & 0.2 & 0.02 & 0.04 & Ae. vexans & $\mathrm{F}$ \\
\hline 35 & & 21 & $10 / 5$ & 11 & 3.6 & 0.36 & 0.72 & Ae. vexans & M \\
\hline 36 & & 25 & $14 / 9$ & & & & & Ae. vexans & $\mathrm{F}$ \\
\hline 37 & & 25 & $14 / 9$ & & & & & Ae. vexans & $\mathrm{F}$ \\
\hline 38 & & 25 & $14 / 9$ & 52 & 7.8 & 0.56 & 0.87 & Ae. vexans & $\mathrm{F}$ \\
\hline 39 & & 25 & 14 / 9 & 35 & 17.9 & 1.28 & 1.99 & Ae. vexans & $\mathrm{F}$ \\
\hline 40 & & 26 & $15 / 10$ & 1 & 0.2 & 0.01 & 0.02 & Cq. perturbans & $\mathrm{F}$ \\
\hline
\end{tabular}


Appendix IX. Continued from previous page.

\begin{tabular}{|c|c|c|c|c|c|c|c|c|c|}
\hline \multirow{2}{*}{ 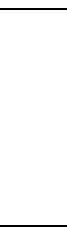 } & \multirow{2}{*}{ Month } & \multirow{2}{*}{ Day } & \multirow{2}{*}{$\begin{array}{c}\text { Days since } \\
\text { first / last } \\
\text { release }\end{array}$} & \multirow{2}{*}{$\begin{array}{l}\text { Trap } \\
\text { no. }\end{array}$} & \multirow{2}{*}{$\begin{array}{l}\mathbf{k m} \text { from } \\
\text { release site }\end{array}$} & \multicolumn{2}{|c|}{$\begin{array}{l}\text { km divided } \\
\text { by no. days }\end{array}$} & \multirow{2}{*}{ Species } & \multirow{2}{*}{ Sex } \\
\hline & & & & & & Min. & Max & & \\
\hline 41 & & 26 & $15 / 10$ & 13 & 4.4 & 0.29 & 0.44 & Ae. vexans & $\mathrm{F}$ \\
\hline 42 & & 27 & $16 / 11$ & 11 & 3.6 & 0.23 & 0.33 & Ae. vexans & $\mathrm{F}$ \\
\hline 43 & & 28 & $17 / 12$ & 52 & 7.8 & 0.46 & 0.65 & Ae. vexans & $\mathrm{F}$ \\
\hline 44 & & 28 & $17 / 12$ & 51 & 26.4 & 1.55 & 2.20 & Cq. perturbans & $\mathrm{F}$ \\
\hline 45 & & 29 & $18 / 13$ & 11 & 3.6 & 0.20 & 0.28 & Ae. vexans & $\mathrm{F}$ \\
\hline 46 & & 29 & $18 / 13$ & & & & & Cq. perturbans & $\mathrm{F}$ \\
\hline 47 & & 30 & $19 / 14$ & 1 & 0.2 & 0.01 & 0.01 & Ae. vexans & $\mathrm{F}$ \\
\hline 48 & & 30 & $19 / 14$ & & & & & Ae. (Oc.) trivittatus & $\mathrm{F}$ \\
\hline 49 & & 30 & $19 / 14$ & 11 & 3.6 & 0.19 & 0.26 & Ae. vexans & $\mathrm{F}$ \\
\hline 50 & & 30 & $19 / 14$ & & & & & Ae. vexans & $\mathrm{F}$ \\
\hline 51 & July & 2 & $21 / 16$ & 1 & 0.2 & 0.01 & 0.01 & Ae. vexans & $\mathrm{F}$ \\
\hline 52 & & 2 & $21 / 16$ & & & & & Ae. vexans & $\mathrm{F}$ \\
\hline 53 & & 2 & $21 / 16$ & & & & & Ae. vexans & $\mathrm{F}$ \\
\hline 54 & & 2 & $21 / 16$ & & & & & Ae. vexans & $\mathrm{F}$ \\
\hline 55 & & 2 & $21 / 16$ & & & & & Ae. vexans & $\mathrm{F}$ \\
\hline 56 & & 2 & $21 / 16$ & & & & & Ae. vexans & $\mathrm{F}$ \\
\hline 57 & & 2 & $21 / 16$ & & & & & Ae. vexans & $\mathrm{F}$ \\
\hline 58 & & 2 & $21 / 16$ & & & & & Ae. vexans & M \\
\hline 59 & & 2 & $21 / 16$ & & & & & Ae. vexans & M \\
\hline 60 & & 2 & $21 / 16$ & & & & & Cq. perturbans & $\mathrm{F}$ \\
\hline 61 & & 2 & $21 / 16$ & & & & & Cx. restuans & $\mathrm{F}$ \\
\hline 62 & & 2 & $21 / 16$ & 13 & 4.4 & 0.21 & 0.23 & Ae. vexans & $\mathrm{F}$ \\
\hline 63 & & 2 & $21 / 16$ & & & & & Ae. vexans & $\mathrm{F}$ \\
\hline 64 & & 2 & $21 / 16$ & & & & & Cx. tarsalis & $\mathrm{F}$ \\
\hline 65 & & 2 & $21 / 16$ & 52 & 7.8 & 0.37 & 0.49 & Ae. vexans & $\mathrm{F}$ \\
\hline 66 & & 2 & $21 / 16$ & & & & & Ae. vexans & $\mathrm{F}$ \\
\hline 67 & & 2 & $21 / 16$ & & & & & Ae. vexans & $\mathrm{F}$ \\
\hline 68 & & 2 & $21 / 16$ & & & & & Ae. vexans & M \\
\hline 69 & & 2 & $21 / 16$ & & & & & Cq. perturbans & $\mathrm{F}$ \\
\hline 70 & & 4 & $23 / 18$ & 1 & 0.2 & 0.01 & 0.01 & Cq. perturbans & $\mathrm{F}$ \\
\hline 71 & & 6 & $25 / 20$ & & & & & Ae. vexans & $\mathrm{F}$ \\
\hline 72 & & 6 & $25 / 20$ & & & & & Ae. vexans & $\mathrm{F}$ \\
\hline 73 & & 6 & $25 / 20$ & & & & & Ae. vexans & $\mathrm{F}$ \\
\hline 74 & & 6 & $25 / 20$ & & & & & Ae. vexans & $\mathrm{M}$ \\
\hline 75 & & 6 & $25 / 20$ & & & & & Unknown & $\mathrm{M}$ \\
\hline 76 & & 7 & $26 / 21$ & 11 & 3.6 & 0.14 & 0.17 & Ae. vexans & $\mathrm{F}$ \\
\hline 77 & & 8 & $27 / 22$ & 52 & 7.8 & 0.29 & 0.35 & Cq. perturbans & $\mathrm{F}$ \\
\hline 78 & & 11 & $30 / 25$ & & & 0.26 & 0.31 & Ae. vexans & $\mathrm{F}$ \\
\hline 79 & & 24 & 43 / 38 & & & 0.18 & 0.21 & Ae. vexans & $\mathrm{F}$ \\
\hline
\end{tabular}


Appendix X. Detailed summary of recaptures by colour (2017) Colours are listed in chronological order of release. All experiments ended on August $28^{\text {th }}$, but only the last day where mosquitoes were recaptured in this period are shown.

\begin{tabular}{|c|c|c|c|c|c|c|c|c|}
\hline Colour & Month & Date & $\begin{array}{l}\text { Trap days } \\
\text { elapsed }\end{array}$ & $\begin{array}{l}\text { Females } \\
\text { recaptured }\end{array}$ & $\begin{array}{c}\text { Males } \\
\text { recaptured }\end{array}$ & $\begin{array}{l}\text { Daily } \\
\text { total }\end{array}$ & Cumulative total & Cumulative \% \\
\hline \multirow{21}{*}{ Orange } & \multirow[t]{21}{*}{ August } & 1 & 0 & & & & - & - \\
\hline & & 2 & 1 & & & & - & - \\
\hline & & 3 & 2 & 9 & 1 & 10 & 10 & 20.4 \\
\hline & & 4 & 3 & 3 & 1 & 4 & 14 & 28.6 \\
\hline & & 5 & 4 & & & & 14 & 28.6 \\
\hline & & 6 & 5 & & & & 14 & 28.6 \\
\hline & & 7 & 6 & & & & 14 & 28.6 \\
\hline & & 8 & 7 & 1 & & 1 & 15 & 30.6 \\
\hline & & 9 & 8 & 1 & 1 & 2 & 17 & 34.7 \\
\hline & & 10 & 9 & & & & 17 & 34.7 \\
\hline & & 11 & 10 & & & & 17 & 34.7 \\
\hline & & 12 & 11 & 3 & & 3 & 20 & 40.8 \\
\hline & & 13 & 12 & 4 & 1 & 5 & 25 & 51.0 \\
\hline & & 14 & 13 & 4 & 1 & 5 & 30 & 61.2 \\
\hline & & 15 & 14 & 6 & & 6 & 36 & 73.5 \\
\hline & & 16 & 15 & & & & 36 & 73.5 \\
\hline & & 17 & 16 & & & & 36 & 73.5 \\
\hline & & 18 & 17 & 2 & & 2 & 38 & 77.6 \\
\hline & & 19 & 18 & 1 & 1 & 2 & 40 & 81.6 \\
\hline & & 20 & 19 & 2 & & 2 & 42 & 85.7 \\
\hline & & 21 & 20 & 5 & 2 & 7 & 49 & 100 \\
\hline
\end{tabular}


Appendix X. Continued from previous page.

\begin{tabular}{lcccccccc}
\hline Color & Month & Date & $\begin{array}{c}\text { Trap days } \\
\text { elapsed }\end{array}$ & $\begin{array}{c}\text { Females } \\
\text { recaptured }\end{array}$ & $\begin{array}{c}\text { Males } \\
\text { recaptured }\end{array}$ & $\begin{array}{c}\text { Daily } \\
\text { total }\end{array}$ & Cumulative total & Cumulative \% \\
\hline \multirow{4}{*}{ August } & 8 & 0 & & & & & - \\
\\
\end{tabular}


Appendix X. Continued from previous page.

\begin{tabular}{|c|c|c|c|c|c|c|c|c|}
\hline Color & Month & Date & $\begin{array}{c}\text { Trap days } \\
\text { elapsed }\end{array}$ & $\begin{array}{c}\text { Females } \\
\text { recaptured }\end{array}$ & $\begin{array}{c}\text { Males } \\
\text { recaptured }\end{array}$ & Daily tota & Cumulative total & Cumulative \% \\
\hline \multirow{5}{*}{ Pink } & August & 19 & 5 & 3 & & 3 & 14 & 66.7 \\
\hline & & 20 & 6 & 1 & & 1 & 15 & 71.4 \\
\hline & & 21 & 7 & 4 & 1 & 5 & 20 & 95.2 \\
\hline & & 22 & 8 & & & & 20 & 95.2 \\
\hline & & 23 & 9 & 1 & & 1 & 21 & 100 \\
\hline \multirow{10}{*}{ Blue } & August & 17 & 0 & & & & - & - \\
\hline & & 18 & 1 & & & & - & - \\
\hline & & 19 & 2 & 1 & 1 & 2 & 2 & 11.1 \\
\hline & & 20 & 3 & 6 & 3 & 9 & 11 & 61.1 \\
\hline & & 21 & 4 & 2 & 1 & 3 & 14 & 77.7 \\
\hline & & 22 & 5 & 1 & & 1 & 15 & 83.3 \\
\hline & & 23 & 6 & & 1 & 1 & 16 & 88.9 \\
\hline & & 24 & 7 & 1 & & 1 & 17 & 94.4 \\
\hline & & 25 & 8 & & & & 17 & 94.4 \\
\hline & & 26 & 9 & 1 & & 1 & 18 & 100 \\
\hline
\end{tabular}


Appendix XI. Daily breakdown recaptured mosquitoes by colour (2017). Traps collections were collected every day. Releases were on August $1^{\text {st }}, 8^{\text {th }}, 14$, and $17^{\text {th }}$. Continued on the next page.

\begin{tabular}{|c|c|c|c|c|c|c|}
\hline \multirow{2}{*}{ Month } & \multirow{2}{*}{ Day } & \multicolumn{5}{|c|}{ Total \# mosquitoes recaptured } \\
\hline & & Orange & Yellow & Pink & Blue & Total \\
\hline \multirow[t]{27}{*}{ August } & 1 & & - & - & - & \\
\hline & 2 & & - & - & - & \\
\hline & 3 & 10 & - & - & - & 10 \\
\hline & 4 & 4 & - & - & - & 4 \\
\hline & 5 & & - & - & - & \\
\hline & 6 & & - & - & - & \\
\hline & 7 & & - & - & - & \\
\hline & 8 & 1 & - & - & - & 1 \\
\hline & 9 & 2 & 4 & - & - & 6 \\
\hline & 10 & & 14 & - & - & 14 \\
\hline & 11 & & 17 & - & - & 17 \\
\hline & 12 & 3 & 4 & - & - & 7 \\
\hline & 13 & 5 & 2 & - & - & 7 \\
\hline & 14 & 5 & 2 & - & - & 7 \\
\hline & 15 & 6 & 16 & 4 & - & 26 \\
\hline & 16 & & 4 & 4 & - & 8 \\
\hline & 17 & & 1 & 2 & - & 3 \\
\hline & 18 & 2 & & 1 & - & 3 \\
\hline & 19 & 2 & 4 & 3 & 2 & 11 \\
\hline & 20 & 2 & 12 & 1 & 9 & 24 \\
\hline & 21 & 7 & 23 & 5 & 3 & 38 \\
\hline & 22 & & & & 1 & 1 \\
\hline & 23 & & & 1 & 1 & 2 \\
\hline & 24 & & & & 1 & 1 \\
\hline & 26 & & 1 & & 1 & 2 \\
\hline & 27 & & & & & \\
\hline & 28 & & & & & \\
\hline Total & & 49 & 104 & 21 & 18 & 192 \\
\hline
\end{tabular}


Appendix XII. Timeline of recaptured mosquitoes in 2017. Traps collections were collected every day. Combined results from all releases are shown. Releases were on August $1^{\text {st }}, 8^{\text {th }}, 14^{\text {th }}$, and $17^{\text {th }}$.

\begin{tabular}{|c|c|c|c|c|c|}
\hline $\begin{array}{l}\text { Trap days } \\
\text { elapsed }\end{array}$ & Females recaptured & Males recaptured & Daily total & Cumulative total & $\begin{array}{l}\text { Cumulative \% of } \\
\text { total recaptured }\end{array}$ \\
\hline 1 & 7 & 1 & 8 & 8 & 4.2 \\
\hline 2 & 20 & 8 & 28 & 36 & 18.8 \\
\hline 3 & 12 & 13 & 25 & 61 & 31.8 \\
\hline 4 & 9 & 5 & 14 & 75 & 39.1 \\
\hline 5 & 6 & 2 & 8 & 83 & 43.2 \\
\hline 6 & 4 & & 4 & 87 & 45.3 \\
\hline 7 & 13 & 10 & 23 & 110 & 57.3 \\
\hline 8 & 5 & 2 & 7 & 117 & 60.9 \\
\hline 9 & 2 & & 2 & 119 & 62.0 \\
\hline 10 & 1 & & 1 & 120 & 62.5 \\
\hline 11 & 6 & 1 & 7 & 127 & 66.1 \\
\hline 12 & 9 & 8 & 17 & 144 & 75.0 \\
\hline 13 & 26 & 2 & 28 & 172 & 89.6 \\
\hline 14 & 6 & & 6 & 178 & 92.7 \\
\hline 15 & & & & 178 & 92.7 \\
\hline 16 & & & & 178 & 92.7 \\
\hline 17 & 2 & & 2 & 180 & 93.8 \\
\hline 18 & 2 & 1 & 3 & 183 & 95.3 \\
\hline 19 & 2 & & 2 & 185 & 96.4 \\
\hline 20 & 5 & 2 & 7 & 192 & 100 \\
\hline
\end{tabular}


Appendix XIII. Number of marked and unmarked mosquitoes captured per $2 \mathrm{~km}$ radius interval from the release site from August $1^{\text {st }}$ to $28^{\text {th }}$, 2017. Numbers of total mosquitoes trapped exclude those marked and recaptured. An asterisk identifies a trap as a miniature light trap (MLT). Continued on the next page.

\begin{tabular}{|c|c|c|c|c|c|c|c|}
\hline $\begin{array}{l}\text { Distance from } \\
\text { release site }(\mathbf{k m})\end{array}$ & Trap no. & $\begin{array}{l}\text { Marked } \\
\text { females }\end{array}$ & $\begin{array}{l}\text { Marked } \\
\text { males }\end{array}$ & $\begin{array}{l}\text { Marked } \\
\text { total }\end{array}$ & $\begin{array}{c}\text { Total females } \\
\text { trapped }\end{array}$ & $\begin{array}{c}\text { Total males } \\
\text { trapped }\end{array}$ & $\begin{array}{c}\text { Total mosquitoes } \\
\text { trapped }\end{array}$ \\
\hline \multirow[t]{5}{*}{$0-2.0$} & 1 & 3 & & 3 & 35 & 11 & 46 \\
\hline & $2 *$ & 9 & 10 & 19 & 31 & 78 & 109 \\
\hline & 3 & 15 & 17 & 32 & 143 & 477 & 620 \\
\hline & $4^{*}$ & 6 & 1 & 7 & 9 & 9 & 18 \\
\hline & $5^{*}$ & 5 & 3 & 8 & 21 & 20 & 41 \\
\hline \multirow[t]{7}{*}{$2.1-4.0$} & $6 *$ & 6 & 1 & 7 & 13 & & 13 \\
\hline & $7 *$ & 2 & & 2 & 7 & 4 & 11 \\
\hline & 8 & 6 & 2 & 8 & 26 & 19 & 45 \\
\hline & 9 & 8 & 2 & 10 & 99 & 70 & 169 \\
\hline & 10 & 3 & & 3 & 14 & 6 & 2 \\
\hline & 11 & 11 & 14 & 25 & 72 & 126 & 198 \\
\hline & 12 & 45 & & 45 & 149 & 24 & 173 \\
\hline \multirow[t]{4}{*}{$4.1-6.0$} & 13 & 8 & & 8 & 67 & 21 & 88 \\
\hline & 14 & 4 & 4 & 8 & 78 & 54 & 132 \\
\hline & 15 & & & & 7 & 3 & 10 \\
\hline & 16 & & & & 28 & 29 & 57 \\
\hline $6.1-8.0$ & 17 & & & & 40 & 27 & 67 \\
\hline \multirow[t]{4}{*}{$8.1-10.0$} & 18 & & & & 1 & 2 & 3 \\
\hline & 19 & & & & 25 & 19 & 44 \\
\hline & 20 & & & & 23 & 53 & 76 \\
\hline & 21 & & & & 40 & 38 & 78 \\
\hline $10.1-12.0$ & 22 & & & & 30 & 21 & 51 \\
\hline \multirow[t]{3}{*}{$12.1-14.0$} & 23 & 1 & & 1 & 104 & 51 & 155 \\
\hline & 24 & & & & 57 & 69 & 126 \\
\hline & 25 & & & & 35 & 20 & 55 \\
\hline
\end{tabular}


Appendix XIII. Continued from previous page.

\begin{tabular}{|c|c|c|c|c|c|c|c|}
\hline $\begin{array}{l}\text { Distance from } \\
\text { release site }(\mathbf{k m})\end{array}$ & Trap no. & $\begin{array}{l}\text { Marked } \\
\text { females }\end{array}$ & $\begin{array}{l}\text { Marked } \\
\text { males }\end{array}$ & $\begin{array}{l}\text { Marked } \\
\text { total }\end{array}$ & $\begin{array}{l}\text { Total females } \\
\text { trapped }\end{array}$ & $\begin{array}{l}\text { Total males } \\
\text { trapped }\end{array}$ & $\begin{array}{c}\text { Total mosquitoes } \\
\text { trapped }\end{array}$ \\
\hline \multirow[t]{7}{*}{$14.1-16.0$} & 26 & & & & 81 & 23 & 104 \\
\hline & 27 & 1 & 1 & 2 & 47 & 98 & 145 \\
\hline & 28 & 2 & & 2 & 70 & 37 & 107 \\
\hline & 29 & & & & 130 & 55 & 185 \\
\hline & 30 & & & & 38 & 14 & 52 \\
\hline & 31 & & & & 20 & 5 & 25 \\
\hline & 32 & & & & 116 & 68 & 184 \\
\hline \multirow[t]{3}{*}{$16.1-18.0$} & 33 & & & & 20 & 17 & 37 \\
\hline & 34 & & & & 12 & 15 & 27 \\
\hline & 35 & & & & 80 & 43 & 123 \\
\hline \multirow[t]{2}{*}{$18.1-20.0$} & 36 & & & & 74 & 79 & 153 \\
\hline & 37 & & & & 171 & 54 & 225 \\
\hline \multirow{6}{*}{$20.1-22.0$} & 38 & & & & 39 & 28 & 67 \\
\hline & 39 & & & & 26 & 9 & 35 \\
\hline & 40 & 1 & & 1 & 169 & 58 & 227 \\
\hline & 41 & & & & 38 & 30 & 68 \\
\hline & 42 & & & & 251 & 100 & 351 \\
\hline & 43 & & & & 45 & 33 & 78 \\
\hline \multirow[t]{6}{*}{$22.1-24.0$} & 44 & & & & 122 & 147 & 269 \\
\hline & 45 & & & & 130 & 3 & 133 \\
\hline & 46 & & & & 134 & 105 & 239 \\
\hline & 47 & & & & 98 & 36 & 134 \\
\hline & 48 & & & & 11 & 3 & 14 \\
\hline & 49 & & & & 82 & 99 & 181 \\
\hline $24.1-26.0$ & 50 & & & & 117 & 51 & 168 \\
\hline $26.1-28.0$ & 51 & 1 & 0 & 1 & 128 & 98 & 226 \\
\hline Total & & 137 & 55 & 192 & 3368 & 2559 & 5944 \\
\hline
\end{tabular}


Appendix XIV. List of recaptured mosquitoes in 2017, ordered by date, trap location, species and sex and release colour. In chronological order of release, $\mathrm{O}$ is orange, $\mathrm{Y}$ is yellow, $\mathrm{P}$ is pink, and $\mathrm{B}$ is blue. Blank entries follow the value of the most recent previous entry.

\begin{tabular}{|c|c|c|c|c|c|c|c|c|c|}
\hline & Month & Day & $\begin{array}{l}\text { Days since } \\
\text { release }\end{array}$ & Trap no. & $\mathbf{k m}$ from release site & $\begin{array}{l}\text { km divided } \\
\text { by no. days }\end{array}$ & Species & Sex & Colour \\
\hline 1 & August & 3 & 2 & 7 & 2.6 & 1.30 & Cs. inornata & $\mathrm{F}$ & $\mathrm{O}$ \\
\hline 2 & & & & 9 & 3.1 & 1.55 & Ae. vexans & $\mathrm{F}$ & \\
\hline 3 & & & & 11 & 3.6 & 1.80 & Ae. vexans & M & \\
\hline 4 & & & & & & & Cs. inornata & $\mathrm{F}$ & \\
\hline 5 & & & & 12 & 3.7 & 1.85 & Cs. inornata & $\mathrm{F}$ & \\
\hline 6 & & & & & & & Cs. inornata & $\mathrm{F}$ & \\
\hline 7 & & & & & & & Cs. inornata & $\mathrm{F}$ & \\
\hline 8 & & & & & & & Cs. inornata & $\mathrm{F}$ & \\
\hline 9 & & & & 14 & 4.7 & 2.35 & Ae. vexans & $\mathrm{F}$ & \\
\hline 10 & & & & & & & CX. tarsalis & $\mathrm{F}$ & \\
\hline 11 & & 4 & 3 & 2 & 0.4 & 0.13 & Cs. inornata & $\mathrm{F}$ & \\
\hline 12 & & & & 3 & 1.0 & 0.33 & Ae. (Oc.) sticticus & M & \\
\hline 13 & & & & 5 & 2.0 & 0.67 & Cs. inornata & $\mathrm{F}$ & \\
\hline 14 & & & & 13 & 4.4 & 1.47 & Cx. tarsalis & $\mathrm{F}$ & \\
\hline 15 & & 8 & 7 & 1 & 0.2 & 0.03 & Ae. (Oc.) dorsalis & $\mathrm{F}$ & \\
\hline 16 & & 9 & 8 & 9 & 3.1 & 0.39 & Cq. perturbans & M & \\
\hline 17 & & & & 10 & 3.3 & 0.41 & Ae. vexans & $\mathrm{F}$ & \\
\hline 18 & & & 1 & 2 & 0.4 & 0.4 & Ae. vexans & $\mathrm{F}$ & $\mathrm{Y}$ \\
\hline 19 & & & & 4 & 1.3 & 1.3 & Ae. vexans & $\mathrm{F}$ & \\
\hline 20 & & & & 12 & 3.7 & 3.7 & Cs. inornata & $\mathrm{F}$ & \\
\hline 21 & & & & 51 & 26.4 & 26.4 & Cq. perturbans & $\mathrm{F}$ & \\
\hline 22 & & 10 & 2 & 1 & 0.2 & 0.1 & Cs. inornata & $\mathrm{F}$ & \\
\hline 23 & & & & 3 & 1.0 & 0.5 & Ae. cinereus & M & \\
\hline 24 & & & & & & & Ae. vexans & $\mathrm{F}$ & \\
\hline 25 & & & & & & & Ae. vexans & $\mathrm{F}$ & \\
\hline 26 & & & & & & & Ae. vexans & M & \\
\hline 27 & & & & & & & Cq. perturbans & $\mathrm{F}$ & \\
\hline
\end{tabular}


Appendix XIV. Continued from previous page.

\begin{tabular}{|c|c|c|c|c|c|c|c|c|c|}
\hline & Month & Day & $\begin{array}{c}\text { Days since } \\
\text { release }\end{array}$ & Trap no. & $\mathbf{k m}$ from release site & $\begin{array}{l}\text { km divided } \\
\text { by no. days }\end{array}$ & Species & Sex & Colour \\
\hline 28 & August & 10 & 2 & 5 & 2.0 & 1.0 & Ae. vexans & $\mathrm{F}$ & $Y$ \\
\hline 29 & & & & & & & Ae. vexans & M & \\
\hline 30 & & & & & & & Cu. minnesotae & M & \\
\hline 31 & & & & 6 & 2.1 & 1.05 & Ae. vexans & $\mathrm{F}$ & \\
\hline 32 & & & & 8 & & 1.35 & Ae. vexans & M & \\
\hline 33 & & & & 12 & & 1.85 & Ae. vexans & $\mathrm{F}$ & \\
\hline 34 & & & & 14 & & 2.35 & Ae. vexans & $\mathrm{F}$ & \\
\hline 35 & & & & & & & Ae. vexans & M & \\
\hline 36 & & 11 & 3 & 3 & 1.0 & 0.33 & Ae. vexans & $\mathrm{F}$ & \\
\hline 37 & & & & & & & Ae. vexans & M & \\
\hline 38 & & & & & & & Ae. vexans & M & \\
\hline 39 & & & & & & & Ae. vexans & M & \\
\hline 40 & & & & & & & Ae. vexans & M & \\
\hline 41 & & & & & & & Cq. perturbans & M & \\
\hline 42 & & & & & & & Cs. inornata & $\mathrm{F}$ & \\
\hline 43 & & & & & & & $C x$. territans & M & \\
\hline 44 & & & & & & & $C x$. territans & M & \\
\hline 45 & & & & 4 & 1.3 & 0.43 & Ae. vexans & $\mathrm{F}$ & \\
\hline 46 & & & & 8 & 2.7 & 0.9 & An. earlei & $\mathrm{F}$ & \\
\hline 47 & & & & 11 & 3.6 & 1.2 & Ae. vexans & M & \\
\hline 48 & & & & & & & $C x$. territans & M & \\
\hline 49 & & & & 13 & 4.4 & 1.47 & Cs. inornata & $\mathrm{F}$ & \\
\hline 50 & & & & & & & Cs. inornata & $\mathrm{F}$ & \\
\hline 51 & & & & 28 & 15.4 & 5.13 & Ae. vexans & $\mathrm{F}$ & \\
\hline 52 & & & & & & & Cx. restuans & $\mathrm{F}$ & \\
\hline 53 & & 12 & 11 & 4 & 1.3 & 0.12 & Cq. perturbans & $\mathrm{F}$ & $\mathrm{O}$ \\
\hline 54 & & & & 12 & 3.7 & 0.34 & An. earlei & $\mathrm{F}$ & \\
\hline 55 & & & & 13 & 4.4 & 0.40 & Cx. restuans & $\mathrm{F}$ & \\
\hline 56 & & & 4 & 6 & 2.1 & 0.52 & Ae. vexans & M & Y \\
\hline 57 & & & & 12 & 3.7 & 0.93 & Cu. minnesotae & $\mathrm{F}$ & \\
\hline
\end{tabular}


Appendix XIV. Continued from previous page.

\begin{tabular}{|c|c|c|c|c|c|c|c|c|c|}
\hline & Month & Day & $\begin{array}{c}\text { Days since } \\
\text { release }\end{array}$ & Trap no. & km from release site & $\begin{array}{l}\text { km divided } \\
\text { by no. days }\end{array}$ & Species & Sex & Colour \\
\hline 58 & August & 12 & 4 & 13 & 4.4 & 1.10 & Ae. vexans & $\mathrm{F}$ & $\mathrm{Y}($ cont'd $)$ \\
\hline 59 & & & & 14 & 4.7 & 1.18 & An. earlei & M & \\
\hline 60 & & 13 & 12 & 3 & 1.0 & 0.08 & Ae. vexans & $\mathrm{F}$ & $\mathrm{O}$ \\
\hline 61 & & & & 9 & 3.1 & 0.26 & Ae. vexans & $\mathrm{F}$ & \\
\hline 62 & & & & 10 & 3.3 & 0.28 & An. earlei & $\mathrm{F}$ & \\
\hline 63 & & & & 11 & 3.6 & 0.30 & Ae. vexans & M & \\
\hline 64 & & & & 12 & 3.7 & 0.31 & Ae. vexans & $\mathrm{F}$ & \\
\hline 65 & & & 5 & 9 & 3.1 & 0.62 & An. earlei & $\mathrm{F}$ & Y \\
\hline 66 & & & & 11 & 3.6 & 0.72 & Ae. vexans & M & \\
\hline 67 & & 14 & 13 & 2 & 0.4 & 0.03 & Ae. vexans & $\mathrm{F}$ & $\mathrm{O}$ \\
\hline 68 & & & & & & & Cs. inornata & $\mathrm{F}$ & \\
\hline 69 & & & & 6 & 2.1 & 0.16 & Ae. vexans & $\mathrm{F}$ & \\
\hline 70 & & & & 8 & 2.7 & 0.21 & Cs. inornata & $\mathrm{F}$ & \\
\hline 71 & & & & 11 & 3.6 & 0.28 & Ae. cinereus & $\mathrm{M}$ & \\
\hline 72 & & & 6 & 4 & 1.3 & 0.22 & Cu. minnesotae & $\mathrm{F}$ & $\mathrm{Y}$ \\
\hline 73 & & & & 12 & 3.7 & 0.62 & Cs. inornata & $\mathrm{F}$ & \\
\hline 74 & & 15 & 14 & 2 & 0.4 & 0.03 & Ae. (Oc.) sticticus & $\mathrm{F}$ & $\mathrm{O}$ \\
\hline 75 & & & & 5 & 2.0 & 0.14 & Cx. territans & $\mathrm{F}$ & \\
\hline 76 & & & & 6 & 2.1 & 0.15 & Ae. (Oc.) sticticus & $\mathrm{F}$ & \\
\hline 77 & & & & 8 & 2.7 & 0.19 & Ae. vexans & $\mathrm{F}$ & \\
\hline 78 & & & & 11 & 3.6 & 0.26 & Ae. (Oc.) sticticus & $\mathrm{F}$ & \\
\hline 79 & & & & 12 & 3.7 & 0.26 & Cs. inornata & $\mathrm{F}$ & \\
\hline 80 & & & 7 & 2 & 0.4 & 0.06 & Ae. vexans & $\mathrm{F}$ & Y \\
\hline 81 & & & & & & & Ae. vexans & M & \\
\hline 82 & & & & & & & Cq. perturbans & $\mathrm{F}$ & \\
\hline 83 & & & & 3 & 1.0 & 0.14 & Ae. cinereus & M & \\
\hline 84 & & & & & & & Ae. vexans & M & \\
\hline 85 & & & & & & & Ae. vexans & M & \\
\hline 86 & & & & & & & Cq. perturbans & M & \\
\hline 87 & & & & & & & Cx. territans & $\mathrm{F}$ & \\
\hline
\end{tabular}


Appendix XIV. Continued from previous page.

\begin{tabular}{|c|c|c|c|c|c|c|c|c|c|}
\hline & Month & Day & $\begin{array}{c}\text { Days since } \\
\text { release }\end{array}$ & Trap no. & km from release site & $\begin{array}{l}\text { km divided } \\
\text { by no. days }\end{array}$ & Species & Sex & Colour \\
\hline 88 & August & 15 & 7 & 6 & 2.1 & 0.30 & Cq. perturbans & $\mathrm{F}$ & $Y($ cont'd) \\
\hline 89 & & & & 8 & 2.7 & 0.39 & Ae. vexans & $\mathrm{F}$ & \\
\hline 90 & & & & & & 0.39 & Ae. vexans & $\mathrm{F}$ & \\
\hline 91 & & & & 9 & 3.1 & 0.44 & An. earlei & $\mathrm{F}$ & \\
\hline 92 & & & & & & 0.44 & Cq. perturbans & M & \\
\hline 93 & & & & 11 & 3.6 & 0.51 & Ae. vexans & $\mathrm{F}$ & \\
\hline 94 & & & & 14 & 4.7 & 0.67 & Ae. vexans & M & \\
\hline 95 & & & & & & & An. earlei & M & \\
\hline 96 & & & 1 & 2 & 0.4 & 0.40 & Ae (Oc.) sticticus & $\mathrm{F}$ & $\mathrm{P}$ \\
\hline 97 & & & & 7 & 2.6 & 2.60 & Cx. tarsalis & $\mathrm{F}$ & \\
\hline 98 & & & & 10 & 3.3 & 3.30 & An. earlei & $\mathrm{F}$ & \\
\hline 99 & & & & 11 & 3.6 & 3.60 & Cq. perturbans & M & \\
\hline 100 & & 16 & 8 & 9 & 3.1 & 0.93 & Cu. minnesotae & $\mathrm{F}$ & $\mathrm{Y}$ \\
\hline 101 & & & & 11 & 3.6 & 0.45 & Ae. vexans & $\mathrm{F}$ & \\
\hline 102 & & & & & & & Ae. vexans & M & \\
\hline 103 & & & & & & & Ae (Oc.) sticticus & $\mathrm{F}$ & \\
\hline 104 & & & & 3 & 1.0 & 0.50 & Ae. vexans & $\mathrm{F}$ & $\mathrm{P}$ \\
\hline 105 & & & & 9 & 3.1 & 1.55 & An. earlei & $\mathrm{F}$ & \\
\hline 106 & & & & 11 & 3.6 & 1.80 & Ae. vexans & M & \\
\hline 107 & & & & 12 & 3.7 & 1.85 & Ae. (Oc.) sticticus & $\mathrm{F}$ & \\
\hline 108 & & 17 & 9 & 9 & 3.1 & 0.34 & Ae. vexans & $\mathrm{F}$ & $\mathrm{Y}$ \\
\hline 109 & & & 3 & 4 & 1.3 & 0.43 & Unknown & M & $\mathrm{P}$ \\
\hline 110 & & & & 11 & 3.6 & 1.20 & Ae. vexans & M & \\
\hline 111 & & 18 & 17 & 6 & 2.1 & 0.12 & Ae. vexans & $\mathrm{F}$ & $\mathrm{O}$ \\
\hline 112 & & & & 12 & 3.7 & 0.22 & Cs. inornata & $\mathrm{F}$ & \\
\hline 113 & & & 4 & & 3.7 & 0.93 & Cs. inornata & $\mathrm{F}$ & $\mathrm{P}$ \\
\hline 114 & & 19 & 18 & 3 & 1.0 & 0.06 & Ae. vexans & M & $\mathrm{O}$ \\
\hline 115 & & & & & & & Cs. inornata & $\mathrm{F}$ & \\
\hline 116 & & & 11 & 9 & 3.1 & 0.28 & Cs. inornata & $\mathrm{F}$ & $\mathrm{Y}$ \\
\hline 117 & & & & 11 & 3.6 & 0.33 & Ae.vexans & M & \\
\hline
\end{tabular}


Appendix XIV. Continued from previous page.

\begin{tabular}{|c|c|c|c|c|c|c|c|c|c|}
\hline & Month & Day & $\begin{array}{c}\text { Days since } \\
\text { release }\end{array}$ & Trap no. & km from release site & $\begin{array}{l}\text { km divided } \\
\text { by no. days }\end{array}$ & Species & Sex & Colour \\
\hline 118 & August & 19 & 11 & 11 & 3.6 & 0.33 & Cs. inornata & $\mathrm{F}$ & $Y($ cont'd) \\
\hline 119 & & & & 13 & 4.4 & 0.40 & Cs. inornata & $\mathrm{F}$ & \\
\hline 120 & & & 5 & 3 & 1.0 & 0.2 & Ae. vexans & $\mathrm{F}$ & $\mathrm{P}$ \\
\hline 121 & & & & & & & Cs. inornata & $\mathrm{F}$ & \\
\hline 122 & & & & 4 & 1.3 & 0.26 & Cs. inornata & $\mathrm{F}$ & \\
\hline 123 & & & 2 & 3 & 1.0 & 0.5 & Ae. vexans & M & $\mathrm{B}$ \\
\hline 124 & & & & 12 & 3.7 & 1.85 & Cs. inornata & $\mathrm{F}$ & \\
\hline 125 & & 20 & 19 & & & 0.19 & Cs. inornata & $\mathrm{F}$ & $\mathrm{O}$ \\
\hline 126 & & & & & & & Cs. inornata & $\mathrm{F}$ & \\
\hline 127 & & & 12 & 2 & 0.4 & 0.03 & Ae. vexans & M & $\mathrm{Y}$ \\
\hline 128 & & & & & & & Ae. vexans & M & \\
\hline 129 & & & & & & & Ae. vexans & M & \\
\hline 130 & & & & & & & Ae. vexans & M & \\
\hline 131 & & & & & & & Ae. vexans & M & \\
\hline 132 & & & & & & & An. earlei & M & \\
\hline 133 & & & & & & & Cx. restuans & M & \\
\hline 134 & & & & 3 & 1.0 & 0.08 & Ae. vexans & $\mathrm{F}$ & \\
\hline 135 & & & & 8 & 2.7 & 0.23 & An. earlei & $\mathrm{F}$ & \\
\hline 136 & & & & 12 & 3.7 & 0.31 & Cs. inornata & $\mathrm{F}$ & \\
\hline 137 & & & & & & & Oc. sticticus & $\mathrm{F}$ & \\
\hline 138 & & & & 14 & 4.7 & 0.39 & Cs. inornata & $\mathrm{F}$ & \\
\hline 139 & & & 6 & 12 & 3.7 & 0.62 & Cs. inornata & $\mathrm{F}$ & $\mathrm{P}$ \\
\hline 140 & & & 3 & 2 & 0.4 & 0.13 & Cs. inornata & M & B \\
\hline 141 & & & & 3 & 1.0 & 0.33 & Cs. inornata & $\mathrm{F}$ & \\
\hline 142 & & & & 5 & 2.0 & 0.67 & Cs. inornata & $\mathrm{F}$ & \\
\hline 143 & & & & 11 & 3.6 & 1.20 & Ae. vexans & $\mathrm{F}$ & \\
\hline 144 & & & & & & & Ae. vexans & M & \\
\hline 145 & & & & & & & Ae. vexans & M & \\
\hline 146 & & & & & 3.7 & 1.23 & Сu. minnesotae & $\mathrm{F}$ & \\
\hline 147 & & & & 12 & & & Cs. inornata & $\mathrm{F}$ & \\
\hline
\end{tabular}


Appendix XIV. Continued from previous page.

\begin{tabular}{|c|c|c|c|c|c|c|c|c|c|}
\hline & Month & Day & $\begin{array}{c}\text { Days since } \\
\text { release }\end{array}$ & Trap no. & km from release site & $\begin{array}{l}\text { km divided } \\
\text { by no. days }\end{array}$ & Species & Sex & Colour \\
\hline 148 & August & 20 & 3 & 13 & 4.4 & 1.47 & Cs. inornata & $\mathrm{F}$ & $\mathrm{B}$ (cont'd) \\
\hline 149 & & 21 & 20 & 3 & 1.0 & 0.05 & Ae. vexans & M & $\mathrm{O}$ \\
\hline 150 & & & & 8 & 2.7 & 0.14 & Cs. inornata & M & \\
\hline 151 & & & & 11 & 3.6 & 0.18 & Ae. vexans & $\mathrm{F}$ & \\
\hline 152 & & & & & & & Cs. inornata & $\mathrm{F}$ & \\
\hline 153 & & & & 12 & 3.7 & 0.19 & Cs. inornata & $\mathrm{F}$ & \\
\hline 154 & & & & 23 & 12.7 & 0.64 & Cs. inornata & $\mathrm{F}$ & \\
\hline 155 & & & & 40 & 21.0 & 1.05 & Ae. vexans & $\mathrm{F}$ & \\
\hline 156 & & & 13 & 2 & 0.4 & 0.03 & Ae. vexans & M & $\mathrm{Y}$ \\
\hline 157 & & & & & & & Cs. inornata & $\mathrm{F}$ & \\
\hline 158 & & & & 3 & 1.0 & 0.08 & Cs. inornata & $\mathrm{F}$ & \\
\hline 159 & & & & 11 & 3.6 & 0.28 & Cs. inornata & $\mathrm{F}$ & \\
\hline 160 & & & & 12 & 3.7 & 0.28 & Cs. inornata & $\mathrm{F}$ & \\
\hline 161 & & & & & & & Cs. inornata & $\mathrm{F}$ & \\
\hline 162 & & & & & & & Cs. inornata & $\mathrm{F}$ & \\
\hline 163 & & & & & & & Cs. inornata & $\mathrm{F}$ & \\
\hline 164 & & & & & & & Cs. inornata & $\mathrm{F}$ & \\
\hline 165 & & & & & & & Cs. inornata & $\mathrm{F}$ & \\
\hline 166 & & & & & & & Cs. inornata & $\mathrm{F}$ & \\
\hline 167 & & & & & & & Cs. inornata & $\mathrm{F}$ & \\
\hline 168 & & & & & & & Cs. inornata & $\mathrm{F}$ & \\
\hline 169 & & & & & & & Cs. inornata & $\mathrm{F}$ & \\
\hline 170 & & & & & & & Cs. inornata & $\mathrm{F}$ & \\
\hline 171 & & & & & & & Cs. inornata & $\mathrm{F}$ & \\
\hline 172 & & & & & & & Cs. inornata & $\mathrm{F}$ & \\
\hline 173 & & & & & & & Cs. inornata & $\mathrm{F}$ & \\
\hline 174 & & & & & & & Cs. inornata & $\mathrm{F}$ & \\
\hline 175 & & & & & & & Cs. inornata & $\mathrm{F}$ & \\
\hline 176 & & & & & & & Cs. inornata & $\mathrm{F}$ & \\
\hline 177 & & & & & & & Си. minnesotae & $\mathrm{F}$ & \\
\hline
\end{tabular}


Appendix XIV. Continued from previous page.

\begin{tabular}{|c|c|c|c|c|c|c|c|c|c|}
\hline & Month & Day & $\begin{array}{l}\text { Days since } \\
\text { release }\end{array}$ & Trap no. & km from release site & $\begin{array}{l}\text { km divided } \\
\text { by no. days }\end{array}$ & Species & Sex & Colour \\
\hline 178 & August & 21 & 13 & 13 & 4.4 & 0.34 & Cs. inornata & $\mathrm{F}$ & $Y($ cont'd) \\
\hline 179 & & & 7 & 5 & 2.0 & 0.29 & Cs. inornata & M & $\mathrm{P}$ \\
\hline 180 & & & & 12 & 3.7 & 0.53 & Cs. inornata & $\mathrm{F}$ & \\
\hline 181 & & & & & 3.7 & 0.53 & Cs. inornata & $\mathrm{F}$ & \\
\hline 182 & & & & & 3.7 & 0.53 & Cs. inornata & $\mathrm{F}$ & \\
\hline 183 & & & & & 3.7 & 0.53 & Cs. inornata & $\mathrm{F}$ & \\
\hline 184 & & & 5 & 11 & 3.6 & 0.72 & Ae. (Oc.) sticticus & M & $\mathrm{B}$ \\
\hline 185 & & & & 12 & 3.7 & 0.74 & Cs. inornata & $\mathrm{F}$ & \\
\hline 186 & & & & 27 & 14.6 & 2.92 & Cs. inornata & $\mathrm{F}$ & \\
\hline 187 & & 22 & 6 & 6 & 2.1 & 0.35 & Cs. inornata & $\mathrm{F}$ & \\
\hline 188 & & 23 & 9 & 1 & 0.2 & 0.02 & Cs. inornata & $\mathrm{F}$ & $\mathrm{P}$ \\
\hline 189 & & & 7 & 27 & 14.6 & 2.09 & Ae. vexans & M & $\mathrm{B}$ \\
\hline 190 & & 24 & 8 & 4 & 1.3 & 0.16 & Cs. inornata & $\mathrm{F}$ & \\
\hline 191 & & 26 & 18 & 3 & 1.0 & 0.06 & An. earlei & $\mathrm{F}$ & Y \\
\hline 192 & & & 10 & 5 & 2.0 & 0.20 & Cs. inornata & $\mathrm{F}$ & $\mathrm{B}$ \\
\hline
\end{tabular}


Appendix XV. Summary of recaptured mosquitoes by species and colour (2017). Species are listed as most to least abundant.

\begin{tabular}{|c|c|c|c|c|c|}
\hline Species & Colour & Total \# recaptured & $\%$ of total \# recaptured & Female \% & Male \% \\
\hline \multirow[t]{5}{*}{ Culiseta inornata } & Orange & 19 & 26.4 & 94.7 & 5.26 \\
\hline & Yellow & 32 & 44.4 & 100 & \\
\hline & Pink & 10 & 13.9 & 90.0 & 10.0 \\
\hline & Blue & 11 & 15.3 & 90.9 & 9.09 \\
\hline & Total & 72 & 100 & 95.8 & 4.20 \\
\hline \multirow[t]{5}{*}{ Aedes vexans } & Orange & 16 & 23.9 & 25.0 & 25.0 \\
\hline & Yellow & 42 & 62.7 & 45.2 & 54.8 \\
\hline & Pink & 4 & 5.97 & 50.0 & 50.0 \\
\hline & Blue & 5 & 7.46 & 20.0 & 80.0 \\
\hline & Total & 67 & 100 & 50.7 & 49.3 \\
\hline \multirow[t]{5}{*}{ Anopheles earlei } & Orange & 2 & 16.7 & 100 & \\
\hline & Yellow & 8 & 66.7 & 62.5 & 37.5 \\
\hline & Pink & 2 & 16.7 & 100 & \\
\hline & Blue & & & & \\
\hline & Total & 12 & 100 & 75.0 & 25.0 \\
\hline \multirow[t]{5}{*}{ Coquillettidia perturbans } & Orange & 2 & 20.0 & 50.0 & 50.0 \\
\hline & Yellow & 7 & 70.0 & 57.1 & 42.9 \\
\hline & Pink & 1 & 10.0 & & 100 \\
\hline & Blue & & & & \\
\hline & Total & 10 & 100 & 50.0 & 50.0 \\
\hline \multirow[t]{5}{*}{ Aedes (Oc.) sticticus } & Orange & 4 & 44.4 & 75.0 & 25.0 \\
\hline & Yellow & 2 & 22.2 & 100 & \\
\hline & Pink & 2 & 22.2 & 100 & \\
\hline & Blue & 1 & 11.1 & & 100 \\
\hline & Total & 9 & 100 & 77.8 & 22.2 \\
\hline
\end{tabular}


Appendix XV. Continued from previous page. One (yellow) male mosquito of an unknown species has been omitted.

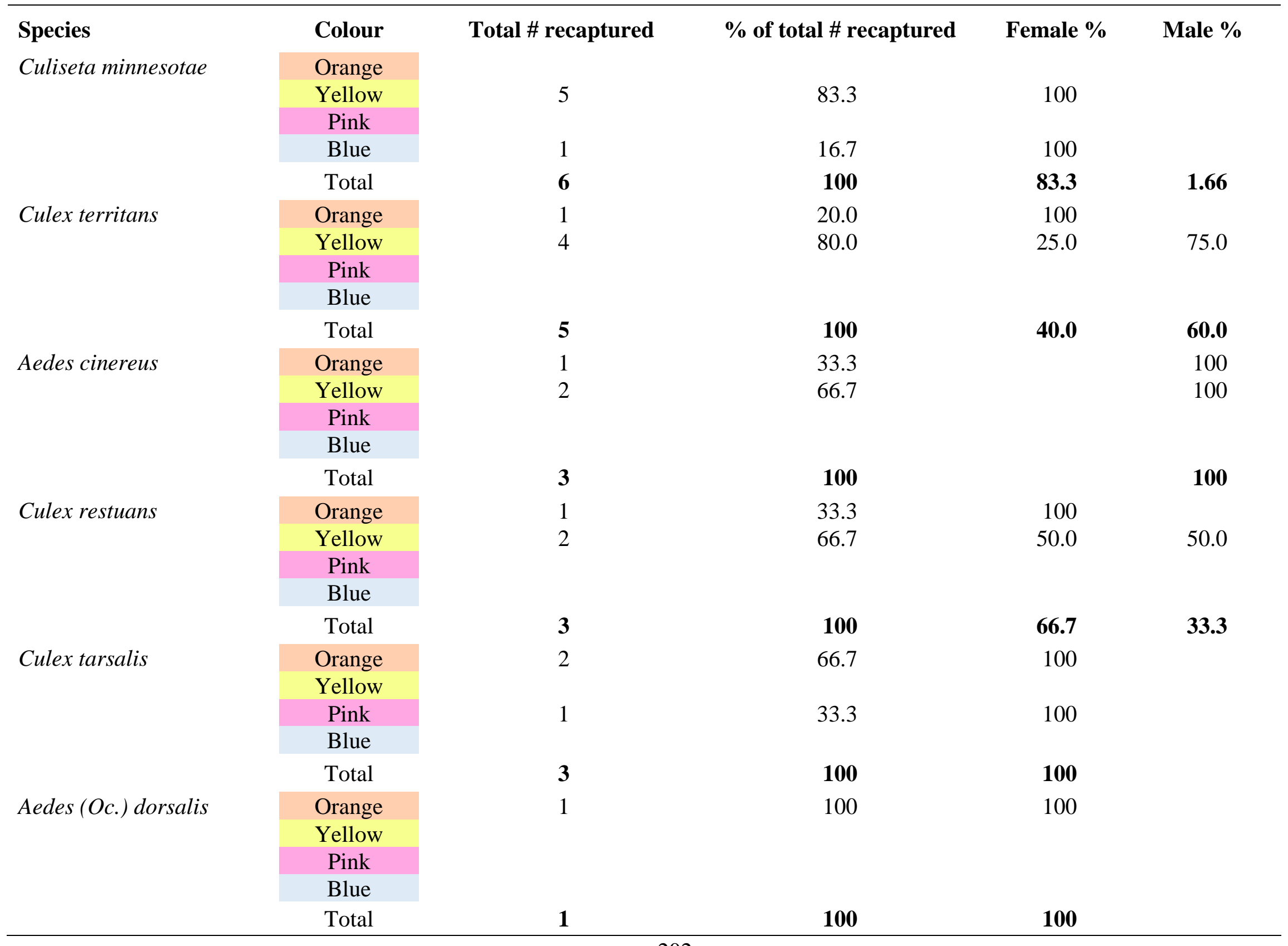


Appendix XVI. Summary of trap site environments. Percentages describe coverage of the two most abundant LULC classes. All coverage, NDVI and NDWI values were calculated within $1 \mathrm{~km}$ of each trap.

\begin{tabular}{|c|c|c|c|c|c|c|}
\hline $\begin{array}{c}\text { Trap } \\
\text { ID }\end{array}$ & $\begin{array}{c}\text { Primary LULC class ( } \% \\
\text { coverage) }\end{array}$ & $\begin{array}{c}\text { Secondary LULC class (\% } \\
\text { coverage) }\end{array}$ & $\begin{array}{l}\text { Distance to nearest } \\
\text { waterway (m) }\end{array}$ & Avg. NDVI & Avg. NDWI & $\begin{array}{l}\text { \% cover of known } \\
\text { larval habitats }\end{array}$ \\
\hline 2 & GRASS (39) & CULTIVATED (20) & 135 & 151 & -0.45 & 45 \\
\hline 4 & GRASS (27) & GROUND (17) & 602 & 129 & -0.29 & 70 \\
\hline 5 & GRASS (26) & CULTIVATED (21) & 3085 & 136 & -0.30 & 34 \\
\hline 6 & RESIDENTIAL (55) & GRASS (18) & 1382 & 132 & -0.31 & 7 \\
\hline 9 & RESIDENTIAL (55) & FOREST (14) & 194 & 140 & -0.36 & $<1$ \\
\hline 10 & FOREST (30) & CULTIVATED (29) & 651 & 150 & -0.43 & 23 \\
\hline 11 & CULTIVATED (47) & GRASS (27) & 6590 & 148 & -0.43 & 25 \\
\hline 12 & RESIDENTIAL (31) & INDUSTRIAL (22) & 821 & 124 & -0.25 & 16 \\
\hline 13 & GRASS (21) & CULTIVATED (20) & 125 & 153 & -0.46 & 23 \\
\hline 17 & CULTIVATED (33) & GRASS (25) & 2941 & 143 & -0.39 & 43 \\
\hline 18 & GRASS (26) & RESIDENTIAL (16) & 3325 & 127 & -0.26 & 51 \\
\hline 19 & RESIDENTIAL (37) & FOREST (28) & 1030 & 150 & -0.43 & 29 \\
\hline 20 & CULTIVATED (27) & RESIDENTIAL (19) & 9142 & 138 & -0.34 & 14 \\
\hline 21 & RESIDENTIAL (60) & GRASS (11) & 1606 & 137 & -0.35 & 10 \\
\hline 22 & RESIDENTIAL (66) & FOREST (14) & 656 & 150 & -0.42 & 2 \\
\hline 23 & RESIDENTIAL (33) & FOREST (25) & 180 & 150 & -0.43 & 38 \\
\hline 24 & RESIDENTIAL (38) & GRASS (16) & 2882 & 127 & -0.27 & 30 \\
\hline 25 & RESIDENTIAL (34) & INDUSTRIAL (27) & 6988 & 126 & -0.26 & 23 \\
\hline 26 & GRASS (43) & RESIDENTIAL (18) & 100 & 139 & -0.37 & 36 \\
\hline 27 & RESIDENTIAL (56) & GRASS (14) & 436 & 143 & -0.38 & 18 \\
\hline
\end{tabular}


Appendix XVII. Summary of mosquitoes trapped, ordered by trap and species according to either trap indices (TI) and harmonic means (HM). Shaded values represent the three traps with the highest value for each species.

\begin{tabular}{|c|c|c|c|c|c|c|c|c|}
\hline \multirow{2}{*}{$\begin{array}{c}\text { Trap } \\
\text { ID }\end{array}$} & \multicolumn{2}{|c|}{ Ae. vexans } & \multicolumn{2}{|c|}{ Cx. tarsalis } & \multicolumn{2}{|c|}{ Cx. restuans } & \multicolumn{2}{|c|}{ Ae. dorsalis } \\
\hline & $\mathrm{TI}$ & $\mathrm{HM}$ & $\mathrm{TI}$ & $\mathrm{HM}$ & TI & $\mathrm{HM}$ & $\mathrm{TI}$ & HM \\
\hline 1 & 33155 & 1352 & 358 & 4.74 & 326 & 13.6 & 298 & 5.5 \\
\hline 2 & 15814 & 695 & 627 & 6.96 & 69 & 2.6 & 150 & 4.7 \\
\hline 3 & 13325 & 404 & 432 & 3.13 & 258 & 8.2 & 26 & 6.1 \\
\hline 4 & 39691 & 1418 & 1053 & 5.42 & 945 & 32.6 & 150 & 7.5 \\
\hline 5 & 2887 & 110 & 407 & 4.22 & 45 & 6.5 & 64 & 4.5 \\
\hline 6 & 10870 & 337 & 764 & 6.23 & 348 & 8.3 & 76 & 5.3 \\
\hline 7 & 32576 & 1898 & 589 & 5.28 & 267 & 23.9 & 81 & 6.6 \\
\hline 8 & 6941 & 254 & 177 & 2.04 & 157 & 5.4 & 36 & 4.3 \\
\hline 9 & 15387 & 755 & 151 & 2.32 & 119 & 4.7 & 25 & 4.2 \\
\hline 10 & 56230 & 2222 & 1671 & 27.8 & 821 & 29.9 & 59 & 5.7 \\
\hline 11 & 6785 & 206 & 629 & 6.37 & 96 & 5.4 & 72 & 5.4 \\
\hline 12 & 5882 & 184 & 320 & 6.30 & 124 & 3.7 & 126 & 12.0 \\
\hline 13 & 58828 & 3152 & 349 & 3.22 & 197 & 10.6 & 145 & 6.9 \\
\hline 14 & 31801 & 1464 & 759 & 10.5 & 668 & 21.1 & 106 & 12.1 \\
\hline 15 & 17436 & 630 & 89 & 1.23 & 188 & 3.3 & 37 & 3.3 \\
\hline 16 & 9313 & 387 & 331 & 3.11 & 311 & 5.4 & 55 & 7.8 \\
\hline 17 & 12333 & 511 & 465 & 6.78 & 144 & 4.9 & 151 & 6.1 \\
\hline 18 & 5541 & 69 & 1211 & 3.94 & 81 & 7.3 & 113 & 8.6 \\
\hline 19 & 31696 & 1285 & 331 & 5.53 & 311 & 19.7 & 54 & 10.0 \\
\hline 20 & 19850 & 933 & 910 & 11.2 & 220 & 5.0 & 86 & 3.3 \\
\hline 21 & 10664 & 71 & 176 & 5.41 & 198 & 11.0 & 25 & 11.9 \\
\hline 22 & 12206 & 725 & 93 & 3.20 & 501 & 22.7 & 396 & 4.3 \\
\hline 23 & 15316 & 919 & 67 & 3.84 & 258 & 9.9 & 27 & 2.8 \\
\hline 24 & 9764 & 180 & 646 & 3.62 & 53 & 2.9 & 94 & 5.4 \\
\hline 25 & 6385 & 308 & 312 & 3.11 & 100 & 3.6 & 41 & 3.8 \\
\hline 26 & 12115 & 645 & 462 & 4.40 & 127 & 3.2 & 418 & 7.0 \\
\hline 27 & 10020 & 416 & 109 & 6.03 & 120 & 7.4 & 34 & 6.2 \\
\hline 28 & 21420 & 668 & 140 & 3.00 & 338 & 8.4 & 29 & 4.1 \\
\hline
\end{tabular}


Appendix XVIII. Coefficients of variation (CVs) of proportional trap totals for each year (used to calculate TI) by species. The mean CV as well as the coefficient of variation of all within-year CVs is also shown for each species.

\begin{tabular}{|c|c|c|c|c|}
\hline Year & Ae. vexans & Cx. tarsalis & Cx. restuans & Ae. dorsalis \\
\hline 2007 & 0.63 & 0.86 & 0.91 & 1.02 \\
\hline 2008 & 1.26 & 1.50 & 1.28 & 1.31 \\
\hline 2009 & 1.28 & 1.18 & 1.26 & 5.29 \\
\hline 2010 & 0.83 & 1.12 & 1.04 & 1.05 \\
\hline 2011 & 1.06 & 2.16 & 1.39 & 1.25 \\
\hline 2012 & 1.67 & 1.19 & 2.40 & 1.16 \\
\hline 2013 & 1.17 & 1.34 & 1.20 & 1.69 \\
\hline 2014 & 1.01 & 0.81 & 0.99 & 3.64 \\
\hline 2015 & 0.73 & 1.13 & 1.13 & - \\
\hline Mean CV & 1.07 & 1.26 & 1.29 & 2.05 \\
\hline $\begin{array}{l}\text { CV of all } \\
\text { yearly CVs }\end{array}$ & 0.30 & 0.32 & 0.35 & 0.76 \\
\hline
\end{tabular}


Appendix XIX. Coefficients of variation (CVs) of the inverses of total trap counts for each year (used to calculate HM) by species. The mean $\mathrm{CV}$ as well as the coefficient of variation of all within-year CVs is also shown for each species.

\begin{tabular}{lrccc}
\hline \multicolumn{1}{c}{ Year } & Ae. vexans & Cx. tarsalis & Cx. restuans & Ae. dorsalis \\
\hline $\mathbf{2 0 0 7}$ & 0.79 & 0.80 & 1.03 & 1.03 \\
$\mathbf{2 0 0 8}$ & 2.21 & 1.24 & 1.25 & 1.00 \\
$\mathbf{2 0 0 9}$ & 2.00 & 1.25 & 1.37 & 5.29 \\
$\mathbf{2 0 1 0}$ & 0.88 & 1.00 & 1.52 & 0.98 \\
$\mathbf{2 0 1 1}$ & 1.10 & 0.86 & 1.20 & 0.91 \\
$\mathbf{2 0 1 2}$ & 1.00 & 1.11 & 1.02 & 0.92 \\
$\mathbf{2 0 1 3}$ & 3.42 & 1.32 & 1.28 & 1.02 \\
$\mathbf{2 0 1 4}$ & 2.02 & 0.84 & 1.20 & 1.64 \\
$\mathbf{2 0 1 5}$ & 0.87 & 1.59 & 1.13 & 1.60 \\
Mean CV & 1.59 & 1.11 & 1.22 & $\mathbf{0 . 9 5}$ \\
CV of all & & & $\mathbf{0 . 1 3}$ & \\
yearly CVs & $\mathbf{0 . 5 6}$ & $\mathbf{0 . 2 4}$ & & \\
\hline
\end{tabular}

